\title{
Iridium-Catalyzed Enantioselective Allylic Vinylation
}

\author{
James Y. Hamilton, David Sarlah, and Erick M. Carreira* \\ Laboratorium für Organische Chemie, ETH Zürich, CH-8093 Zürich, Switzerland \\ *E-mail: carreira@org.chem.ethz.ch
}

\section{Supporting Information}

\section{General:}

Unless otherwise noted, all reactions were carried out under an ambient atmosphere, and all reagents were purchased from commercial suppliers and used without further purification. Analytical thin layer chromatography (TLC) was performed on Merck silica gel 60 F254 TLC glass plates and visualized with $254 \mathrm{~nm}$ light and potassium permanganate staining solutions followed by heating. Purification of reaction products was carried out by flash chromatography using Brunschwig silica 32-63, 60A under 0.3-0.5 bar pressure. $\mathrm{AgNO}_{3}$ impregnated silica preparative TLC was performed as described in literature. ${ }^{1}$ Allylic alcohols were prepared by the reaction of the corresponding aldehyde with vinyl magnesium bromide. Analytical data were in accordance with previously reported values. ${ }^{2-5}$ Potassium alkenyltrifluoroborates were prepared from the corresponding boronic acids or their esters by published procedures. ${ }^{6-9}{ }^{1} \mathrm{H}-\mathrm{NMR}$ spectra were recorded on Bruker AV $400 \mathrm{MHz}$ or VARIAN Mercury $300 \mathrm{MHz}$ spectrometers and are reported in ppm with the solvent resonance employed as the internal standard $\left(\mathrm{CDCl}_{3}\right.$ at $\left.7.26 \mathrm{ppm}\right)$. Peaks are reported as $(\mathrm{s}=$ singlet, $\mathrm{d}=$ doublet, $\mathrm{t}=$ triplet, $\mathrm{q}=$ quartet, $\mathrm{m}=$ multiplet or unresolved, $\mathrm{br}=$ broad signal, coupling constant(s) in $\mathrm{Hz}$, integration). ${ }^{13} \mathrm{C}-\mathrm{NMR}$ spectra were recorded with ${ }^{1} \mathrm{H}$-decoupling on Bruker AV $100 \mathrm{MHz}$ or VARIAN Mercury $75 \mathrm{MHz}$ spectrometers and are reported in ppm with the solvent resonance employed as the internal standard ( $\mathrm{CDCl}_{3}$ at $\left.77.0 \mathrm{ppm}\right)$. Infrared spectra were measured neat on a Perkin-Elmer spectrum BX FT-IR spectrometer. The peaks are reported as absorption maxima $\left(\mathrm{n}, \mathrm{cm}^{-1}\right)$. High resolution mass spectral data were obtained at the mass spectrometry service operated by the Laboratory of Organic Chemistry at the ETHZ on a VG-TRIBRID for electron impact ionization (EI) and Varian IonSpec spectrometer (ESI) and are reported as $(\mathrm{m} / \mathrm{z})$. Enantiomeric excesses were determined on a Jasco2080Plus supercritical fluid chromatography (SFC). Optical rotations were measured with a Jasco DID-1000 Polarimeter, $10 \mathrm{~cm}, 1 \mathrm{~mL}$ cell.

\section{Table of Contents}

Synthesis of Phosphoramidite Ligand (S)-L $\quad$ S2

Optimization Studies $\quad$ S3

Synthesis and Characterization of Products $\quad$ S7

Synthesis of (-)-Nyasol and (-)-Hinokiresinol $\quad$ S16

References $\quad$ S18

SFC Data $\quad$ S19

NMR Spectral Data $\quad$ S45 


\section{Synthesis of Phosphoramidite Ligand $(S)-\mathrm{L}^{2}$}

A Schlenk flask was charged with (S)-BINOL (10.0 g, $34.9 \mathrm{mmol}, 1.00$ equiv). $\mathrm{PCl}_{3}$ (45.8 mL, $524 \mathrm{mmol}, 15.0$ equiv) and a catalytic amount of $N, N$-Dimethylformamide $(81.0 \mu \mathrm{l}, 1.05 \mathrm{mmol}, 0.03$ equiv) were added, and the reaction mixture was stirred at $50{ }^{\circ} \mathrm{C}$ for $30 \mathrm{~min}$. The initially heterogeneous mixture turned into a homogenous solution. After cooling to $23{ }^{\circ} \mathrm{C}$, excess $\mathrm{PCl}_{3}$ was evaporated into a cold-finger-trap in vacuo and quenched with saturated aqueous $\mathrm{NaHCO}_{3} .5 \mathrm{~mL}$ of toluene was added, and remaining $\mathrm{PCl}_{3}$ was azeotropically removed. The resulting phosphorochloridite (air-and moisture-sensitive!) was redissolved in THF (200 mL). In a separate Schlenk flask under argon, iminostilbene ( $7.44 \mathrm{~g}, 38.5 \mathrm{mmol}, 1.1$ equiv) was dissolved in THF (200 $\mathrm{mL})$ and deprotonated at $-78^{\circ} \mathrm{C}$ by a slow addition of $n$-BuLi ( $21.6 \mathrm{~mL}, 1.05$ equiv, $1.6 \mathrm{M}$ solution in hexanes). The resulting deep blue solution was stirred at $-78^{\circ} \mathrm{C}$ for 1 hour before the phosphorochloridite solution was slowly added via cannula. The resulting mixture was stirred at $-78{ }^{\circ} \mathrm{C}$ and gradually $23^{\circ} \mathrm{C}$ whiling stirring for $10 \mathrm{~h}$. After completion of the reaction, as determined by TLC, the solvents were evaporated in vacuo. Purification of the residue by flash chromatography $\left(\mathrm{SiO}_{2}\right.$; hexane/toluene $\left.2: 1\right)$ yielded the desired product as a white powder. Yield: $10.8 \mathrm{~g}$ (61 \%). Kept under an inert atmosphere, in the dark, for long-term storage.

${ }^{1} \mathrm{H}-\mathrm{NMR}(400 \mathrm{MHz}$ CDCl3) $\delta=7.99(\mathrm{~d}, \mathrm{~J}=8.8 \mathrm{~Hz}, 1 \mathrm{H}), 7.91(\mathrm{~d}, \mathrm{~J}=8.2 \mathrm{~Hz}, 1 \mathrm{H}), 7.76(\mathrm{~d}, \mathrm{~J}=8.1 \mathrm{~Hz}, 1 \mathrm{H}), 7.62(\mathrm{~d}, \mathrm{~J}=$ $8.8 \mathrm{~Hz}, 1 \mathrm{H}), 7.45-7.34(\mathrm{~m}, 3 \mathrm{H}), 7.30-7.15(\mathrm{~m}, 9 \mathrm{H}), 7.12-7.09(\mathrm{~m}, 1 \mathrm{H}), 7.01-6.90(\mathrm{~m}, 3 \mathrm{H}), 6.85(\mathrm{~d}, \mathrm{~J}=8.8 \mathrm{~Hz}$, $1 \mathrm{H}), 6.53(\mathrm{td}, \mathrm{J}=7.7,1.5 \mathrm{~Hz}, 1 \mathrm{H}) ;{ }^{13} \mathrm{C}-\mathrm{NMR}(100 \mathrm{MHz}$; CDCl3) $\delta=149.95,149.86,148.7,143.1,142.5,136.5$, $135.2,132.9,132.2,131.53,131.44,131.34,130.31,130.20,129.16,129.07,129.05,129.00,128.97,128.90$, $128.55,128.40,128.30,127.9,127.1,126.80,126.70,126.15,126.04,125.6,124.8,124.3,122.1,121.5 ;{ }^{31} \mathrm{P}-$ NMR (161 MHz; CDCl3) $\delta=137.88$ (s). 


\section{Optimization Studies}
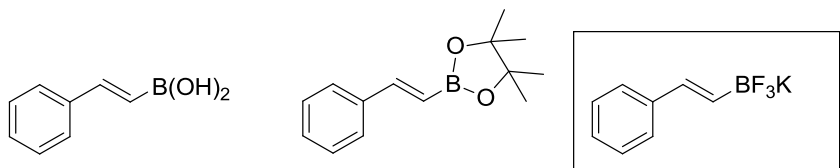

The initial vinylation screening was performed using the three reagents shown above. However, only the trifluoroborate showed some reactivity at the beginning of these studies and was, therefore, used in subsequent optimization studies.

\section{Identification of Trifluoroborate Activator}

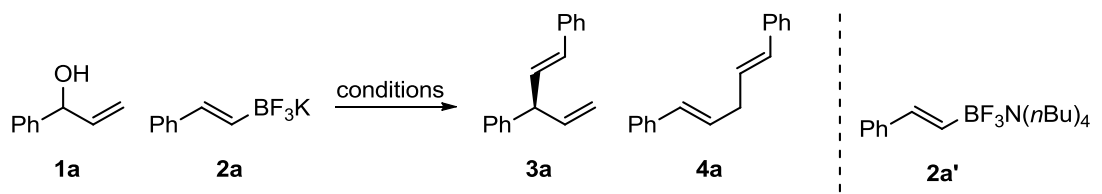

Standard Procedure: allylic alcohol 1a $\left(0.25 \mathrm{mmol}, 1.0\right.$ equiv), $\left[\{\mid \mathrm{Ir}(\operatorname{cod}) \mathrm{Cl}\}_{2}\right],[\mid \mathrm{r}] /(\boldsymbol{S})-\mathrm{L}=1: 2$, promoter $(\mathbf{A}:$ di- $n$ butyl phosphoric acid or $\left.\mathbf{B}: n \mathrm{Bu}_{4} \mathrm{NHSO}_{4}\right)$, solvent $(0.5 \mathrm{~mL}), 25{ }^{\circ} \mathrm{C}, 24 \mathrm{~h}$. Yields were determined by ${ }^{1} \mathrm{H}-\mathrm{NMR}$ by using 1,3,5-trimethoxybenzene as the internal standard.

Table S1

\begin{tabular}{|c|c|c|c|c|c|c|c|c|}
\hline entry & $\begin{array}{c}\text { cat. } \\
\text { (mol\%) }\end{array}$ & $\begin{array}{c}\mathbf{2 a} \\
\text { (equiv) }\end{array}$ & $\begin{array}{l}\text { promoter } \\
\text { (mol\%) }\end{array}$ & $\begin{array}{c}\mathrm{RBF}_{3} \mathrm{~K} \text { activator } \\
\text { (equiv) }\end{array}$ & solvent & $\begin{array}{l}\text { yield } \\
(\%)\end{array}$ & $3 a: 4 a$ & $\begin{array}{l}\text { ee } \\
(\%)\end{array}$ \\
\hline 1 & 2 & 1 & $A(50)$ & KF (1.0) & Acetone & N.R. & - & - \\
\hline 2 & 2 & 1 & $A(150)$ & KF (1.0) & Acetone & N.R. & - & - \\
\hline 3 & 2 & 1 & $A(50)$ & $\mathrm{KHF}_{2}(1.0)$ & Acetone & N.R. & - & - \\
\hline 4 & 2 & 1 & A (50) & TBAF (1.0) & Acetone & N.R. & - & - \\
\hline 5 & 2 & 1 & $A(150)$ & TBAF (1.0) & Acetone & N.R. & - & - \\
\hline 6 & 2 & 1 & A (50) & $\mathrm{Et}_{3} \mathrm{~N}-3 \mathrm{HF}(0.5)$ & Acetone & 6 & - & - \\
\hline 7 & 2 & 1 & A (50) & $\mathrm{Et}_{3} \mathrm{~N}-3 \mathrm{HF}(1.0)$ & Acetone & 11 & - & - \\
\hline 8 & 2 & 1 & $A(50)$ & TMSF & Acetone & 15 & $>50: 1$ & 99 \\
\hline 9 & 2 & 1 & $A(50)$ & HF (1.0) & Acetone & 24 & $>50: 1$ & 99 \\
\hline 10 & 2 & $1\left(2 a^{\prime}\right)$ & A (50) & $\mathrm{Et}_{3} \mathrm{~N}-3 \mathrm{HF}(1.0)$ & Acetone & 28 & $>50: 1$ & 98 \\
\hline 11 & 2 & $1\left(2 a^{\prime}\right)$ & $\mathbf{A}(50)$ & HF (1.0) & Acetone & 40 & $>50: 1$ & 98 \\
\hline 12 & 4 & 2 & B (10) & TMSCl (2.0) & Dioxane & $34(19)^{a}$ & $>50: 1$ & 97 \\
\hline 13 & 4 & 2 & B (10) & TMSCl (4.0) & Dioxane & $<5(72)^{a}$ & - & - \\
\hline 14 & 4 & 2 & B (10) & $\mathrm{SiCl} 4$ (2.0) & Dioxane & $11(58)^{a}$ & $<1: 50$ & - \\
\hline 15 & 4 & 2 & B (10) & $\mathrm{SiCl} 4$ (2.0) & Dioxane & $12(67)^{a}$ & $<1: 50$ & - \\
\hline
\end{tabular}

${ }^{a}$ Yield of undesired cinnamyl chloride. 


\section{Effect of Solvent on Reaction:}

Standard Procedure: allylic alcohol 1a (0.25 mmol, 1.0 equiv), [\{lr(cod)Cl$\left.\}_{2}\right](2 \mathrm{~mol} \%),(S)-\mathrm{L}(8 \mathrm{~mol} \%)$, potassium styryltrifluoroborate 2a $(0.25 \mathrm{mmol}, 1.0$ equiv), promoter ( $50 \mathrm{~mol} \%$; $\mathbf{A}$ : di-n-butyl phosphoric acid or $\mathbf{B}$ :

$\left.n \mathrm{Bu}_{4} \mathrm{NHSO}_{4}\right), \mathrm{HF}\left(0.25 \mathrm{mmol}, 1.0\right.$ equiv, $50 \%$ aq.) solvent $(0.5 \mathrm{~mL}), 25{ }^{\circ} \mathrm{C}, 24 \mathrm{~h}$. Yields were determined by ${ }^{1} \mathrm{H}-$ NMR by using 1,3,5-trimethoxybenzene as the internal standard.

Table S2

\begin{tabular}{cccccc}
\hline entry & Promoter & solvent & yield (\%) & 3a:4a & ee (\%) \\
\hline 1 & A & DCE & 22 & $9: 1$ & 97 \\
2 & A & DMF & $<5$ & - & - \\
3 & A & THF & $<5$ & - & - \\
4 & A & Acetone & 24 & $>50: 1$ & 99 \\
5 & B & Acetone & 53 & $>50: 1$ & 98 \\
6 & B & MeCN & 31 & $0.7: 1$ & 97 \\
7 & B & DME & 22 & $20: 1$ & 97 \\
8 & B & MeNO & 40 & $0.6: 1$ & 97 \\
9 & B & Dioxane & 60 & $>50: 1$ & 98 \\
\hline
\end{tabular}

\section{Effect of Loading of $n \mathrm{Bu}_{4} \mathrm{NHSO}_{4}$ on Reaction}

Standard Procedure: allylic alcohol 1a (0.25 mmol, 1.0 equiv), [ $\left.\{\mid \mathrm{r}(\operatorname{cod}) \mathrm{Cl}\}_{2}\right]$ (2 mol\%), (S)-L (8 mol\%), potassium styryltrifluoroborate $2 \mathrm{a}\left(0.25 \mathrm{mmol}, 1.0\right.$ equiv), $\mathrm{HF}\left(0.25 \mathrm{mmol}, 1.0\right.$ equiv, $50 \%$ aq.) solvent $(0.5 \mathrm{~mL}), 25^{\circ} \mathrm{C}, 24 \mathrm{~h}$. Yields were determined by ${ }^{1} \mathrm{H}$-NMR by using 1,3,5-trimethoxybenzene as the internal standard.

Table S3

\begin{tabular}{cccccc}
\hline entry & $\begin{array}{c}n \mathrm{Bu}_{4} \mathrm{NHSO}_{4} \\
(\mathrm{~mol} \%)\end{array}$ & Solvent & $\begin{array}{c}\text { yield } \\
(\%)\end{array}$ & $3 \mathrm{a}: 4 \mathrm{a}$ & $\begin{array}{c}\text { ee } \\
(\%)\end{array}$ \\
\hline 1 & 50 & Acetone & 56 & $>50: 1$ & 98 \\
2 & 40 & Acetone & 53 & $>50: 1$ & 99 \\
3 & 30 & Acetone & 53 & $>50: 1$ & 99 \\
4 & 20 & Acetone & 52 & $>50: 1$ & 99 \\
5 & 10 & Acetone & 53 & $>50: 1$ & 99 \\
6 & 50 & Dioxane & 57 & $>50: 1$ & 98 \\
7 & 40 & Dioxane & 59 & $>50: 1$ & 99 \\
8 & 30 & Dioxane & 57 & $>50: 1$ & 99 \\
9 & 20 & Dioxane & 59 & $>50: 1$ & 99 \\
10 & 10 & Dioxane & 60 & $>50: 1$ & 99 \\
\hline
\end{tabular}




\section{Effect of Temperature on Reaction}

Standard Procedure: allylic alcohol 1a $\left(0.25 \mathrm{mmol}, 1.0\right.$ equiv), [ $\left.\{\mathrm{Ir}(\mathrm{cod}) \mathrm{Cl}\}_{2}\right](2 \mathrm{~mol} \%),(\mathrm{S})-\mathrm{L}(8 \mathrm{~mol} \%)$, potassium styryltrifluoroborate $2 \mathrm{a}$ ( $0.25 \mathrm{mmol}, 1.0$ equiv), $n \mathrm{Bu}_{4} \mathrm{NHSO}_{4}$ ( $\left.50 \mathrm{~mol} \%\right), \mathrm{HF}(0.25 \mathrm{mmol}, 1.0$ equiv, $50 \%$ aq.) acetone $(0.5 \mathrm{~mL}), 24 \mathrm{~h}$. Yields were determined by ${ }^{1} \mathrm{H}$-NMR by using 1,3,5-trimethoxybenzene as the internal standard.

Table S4

\begin{tabular}{ccccc}
\hline entry & $\mathrm{T}\left({ }^{\circ} \mathrm{C}\right)$ & $\begin{array}{c}\text { yield } \\
(\%)\end{array}$ & 3a:4a & $\begin{array}{c}\text { ee } \\
(\%)\end{array}$ \\
\hline 1 & -20 & 30 & $>50: 1$ & 99 \\
2 & 4 & 53 & $>50: 1$ & 99 \\
3 & 25 & 53 & $>50: 1$ & 98 \\
4 & 50 & 50 & $>50: 1$ & 97 \\
\hline
\end{tabular}

\section{Effect of Loading of Ir-catalyst and Ir to (S)-L Ratio on Reaction}

Standard Procedure: allylic alcohol $1 \mathrm{a}\left(0.25 \mathrm{mmol}, 1.0\right.$ equiv), $n \mathrm{Bu}_{4} \mathrm{HNSO}_{4}$ (10 mol\%), $\mathrm{HF}(50 \%$ aq., equimolar to 2a), dioxane $(0.5 \mathrm{~mL}), 25^{\circ} \mathrm{C}, 24 \mathrm{~h}$. Yields were determined by ${ }^{1} \mathrm{H}$-NMR by using 1,3,5-trimethoxybenzene as the internal standard.

Table S5

\begin{tabular}{ccccccc}
\hline entry & $\begin{array}{c}{\left[\{\mid \mathrm{I}(\mathrm{cod}) \mathrm{Cl}\}_{2}\right]} \\
(\mathrm{mol} \%)\end{array}$ & $\begin{array}{c}(\mathrm{S})-\mathbf{L} \\
(\mathrm{mol} \%)\end{array}$ & $\begin{array}{c}2 \mathrm{a} \\
(\text { equiv })\end{array}$ & $\begin{array}{c}\text { yield } \\
(\%)\end{array}$ & $3 \mathrm{3a:4a}$ & $\begin{array}{c}\text { ee } \\
(\%)\end{array}$ \\
\hline 1 & 2 & 8 & 2 & 52 & $23: 1$ & 99 \\
2 & 3 & 12 & 2 & 75 & $>50: 1$ & 99 \\
3 & 4 & 16 & 2 & 89 & $>50: 1$ & 99 \\
4 & 4 & 12 & 2 & 58 & $>50: 1$ & 98 \\
5 & 5 & 10 & 2 & $<5$ & - & - \\
6 & 4 & 16 & $1+1(6 \mathrm{~h})^{\mathrm{a}}$ & 68 & $>50: 1$ & 99 \\
7 & $2+2(6 \mathrm{~h})^{\mathrm{a}}$ & $8+8(6 \mathrm{~h})^{\mathrm{a}}$ & $1+1(6 \mathrm{~h})^{\mathrm{a}}$ & 69 & $>50: 1$ & 99 \\
8 & 4 & 16 & $1+1(12 \mathrm{~h})^{\mathrm{a}}$ & 67 & $>50: 1$ & 99 \\
9 & $2+2(12 \mathrm{~h})^{\mathrm{a}}$ & $8+8(12 \mathrm{~h})^{\mathrm{a}}$ & $1+1(12 \mathrm{~h})^{\mathrm{a}}$ & 64 & $>50: 1$ & 99 \\
\hline
\end{tabular}

${ }^{\mathrm{a}}$ Sequential addition of $\mathbf{2 a}$ and $\mathrm{HF}$ at indicated time intervals. 


\section{Effect of the Amount of Styryltrifluoroborate 2a and its Sequential Addition}

Standard Procedure: allylic alcohol 1a $\left.(0.25 \mathrm{mmol}, 1.0 \text { equiv), [\{lr(cod)Cl}\}_{2}\right],[\mid \mathrm{r}] /(S)-\mathrm{L}=1: 2$, promoter (A: di- $n$ butyl phosphoric acid or $\left.\mathbf{B}: n \mathrm{Bu}_{4} \mathrm{NHSO}_{4}\right), \mathrm{HF}\left(50 \%\right.$ aq., equimolar to $2 \mathrm{a}$ ), solvent $(0.5 \mathrm{~mL}), 25^{\circ} \mathrm{C}, 24 \mathrm{~h}$. Yields were determined by ${ }^{1} \mathrm{H}$-NMR by using 1,3,5-trimethoxybenzene as the internal standard.

\section{Table S6}

\begin{tabular}{cccccccc}
\hline entry & $\begin{array}{c}\text { cat. } \\
\text { (mol\%) }\end{array}$ & $\begin{array}{c}\text { 2a } \\
\text { (equiv) }\end{array}$ & $\begin{array}{c}\text { promoter } \\
\text { (mol\%) }\end{array}$ & solvent & $\begin{array}{c}\text { yield } \\
(\%)\end{array}$ & 3a:4a & $\begin{array}{c}\text { ee } \\
(\%)\end{array}$ \\
\hline 1 & 2 & 1 & A (50) & Acetone & 24 & $>50: 1$ & 99 \\
2 & 2 & 1.5 & A (50) & Acetone & 31 & $19: 1$ & 99 \\
3 & 2 & 2 & A (50) & Acetone & 39 & $14: 1$ & 97 \\
4 & 2 & 1 & B (50) & Acetone & 53 & $>50: 1$ & 98 \\
5 & 2 & $1+1(6 \mathrm{~h})^{\text {a }}$ & B (50) & Acetone & 69 & $20: 1$ & 98 \\
6 & 2 & $1+1(12 \mathrm{~h})^{\mathrm{a}}$ & B (50) & Acetone & 60 & $20: 1$ & 98 \\
7 & 2 & 2 & B (50) & Acetone & 73 & $20: 1$ & 98 \\
8 & 4 & 1.5 & B (10) & Dioxane & 74 & $>50: 1$ & 99 \\
9 & 4 & $1+1(6 \mathrm{~h})^{\mathrm{a}}$ & B (10) & Dioxane & 68 & $>50: 1$ & 99 \\
10 & 4 & $1+1(12 \mathrm{~h})^{\mathrm{a}}$ & B (10) & Dioxane & 67 & $>50: 1$ & 99 \\
11 & 4 & 2 & B (10) & Dioxane & 89 & $>50: 1$ & 99
\end{tabular}

${ }^{\mathrm{a}}$ Sequential addition of $\mathbf{2 a}$ and HF at indicated time intervals. 


\section{Synthesis and Characterization of Products}

\section{General Procedure for Ir-Catalyzed Allylic Vinylation}

$\left[\left\{\mid r(\operatorname{cod}) \mathrm{Cl}_{2}\right](6.7 \mathrm{mg}, 10.0 \mu \mathrm{mol}, 0.04\right.$ equiv) and $(S)-\mathrm{L}(20.3 \mathrm{mg}, 40.0 \mu \mathrm{mol}, 0.16$ equiv) were dissolved in 1,4dioxane $(0.5 \mathrm{~mL})$ in a screw capped polypropylene vial and vigorously stirred for $15 \mathrm{~min}$. To the resulting dark red solution, allylic alcohol 1 ( $0.25 \mathrm{mmol}, 1.0$ equiv), potassium alkenyltrifluoroborate $\mathbf{2}$ ( $0.50 \mathrm{mmol}, 2.0$ equiv), $n \mathrm{Bu}_{4} \mathrm{NHSO}_{4}\left(8.5 \mathrm{mg}, 25 \mu \mathrm{mol}, 0.1\right.$ equiv), and $\mathrm{HF}\left(17 \mu \mathrm{L}, 0.50 \mathrm{mmol}, 2.0\right.$ equiv, $50 \%$ in $\left.\mathrm{H}_{2} \mathrm{O}\right)$ were added. The resulting heterogeneous orange mixture was stirred at room temperature for $4 \mathrm{~h}$. The reaction mixture was concentrated under a reduced pressure and purified by flash chromatography to afford the product.

\section{Preparation of Racemic Standards}

Racemic samples were obtained by using the above-mentioned procedure, however racemic ligand (rac)-L1 ${ }^{2}$ (16.3 mg, $40.0 \mu \mathrm{mol}, 0.16$ equiv) was used instead of (S)-L.<smiles></smiles>

(rac)-L1

\section{(R,E)-penta-1,4-diene-1,3-diyldibenzene (3a)}<smiles>C=CC(C=Cc1ccccc1)(c1ccccc1)c1ccccc1</smiles>

The corresponding compound was prepared following the general procedure by using 1-phenylprop-2-en-1-ol $\mathbf{1 a}$ and potassium $(E)$-styryltrifluoroborate $\mathbf{2 a}$. The product was isolated by flash chromatography (hexane/EtOAc=99/1) to afford the product as a colorless oil $(47.4 \mathrm{mg}, 86 \%) ;{ }^{1} \mathrm{H}-\mathrm{NMR}\left(300 \mathrm{MHz}, \mathrm{CDCl}{ }_{3}\right): \delta=$ $7.44-7.16(\mathrm{~m}, 10 \mathrm{H}), 6.51-6.36(\mathrm{~m}, 2 \mathrm{H}), 6.13(\mathrm{ddd}, J=17.0,10.2,6.4 \mathrm{~Hz}, 1 \mathrm{H}), 5.26-5.10(\mathrm{~m}, 2 \mathrm{H}), 4.24(\mathrm{t}, J=$ $6.4 \mathrm{~Hz}, 1 \mathrm{H}) ;{ }^{13} \mathrm{C}-\mathrm{NMR}\left(75 \mathrm{MHz}, \mathrm{CDCl}_{3}\right): \delta=142.6,140.0,137.3,131.7,130.6,128.5,128.4,128.0,127.2,126.5$, 126.2, 115.6, 52.4; IR (neat): 3081, 3059, 3025, 2977, 2924, 2855, 1634, 1599, 1492, 1449, 1407, 1073, 1029, $965,915,742,691 \mathrm{~cm}^{-1}$; HRMS (EI): $\mathrm{m} / z$ calcd for $\mathrm{C}_{17} \mathrm{H}_{16}[\mathrm{M}]^{+}$220.1252, found 220.1247; SFC: Daicel Chiracel $\mathrm{OJ}-\mathrm{H}, 2 \% \mathrm{MeOH}, 2.0 \mathrm{~mL} / \mathrm{min}, 25{ }^{\circ} \mathrm{C}, 200 \mathrm{~nm} ;>99 \%$ ee $\left(t_{R}(\right.$ major $)=20.1 \mathrm{~min}, \mathrm{t}_{\mathrm{R}}($ minor $\left.)=22.8 \mathrm{~min}\right) ;[\alpha]^{27}{ }_{\mathrm{D}}=$ $-10.3\left(\mathrm{c}=2.0, \mathrm{CHCl}_{3}\right)$.

Characterization data of this compound were in accordance with previously reported values. ${ }^{10}$ The absolute stereochemistry of the reported compound was determined $(S)$ with specific rotation $[\alpha]_{D}^{20}=+3.3(\mathrm{c}=0.60$, $\mathrm{CHCl}_{3}$ ), optical purity of $94 \%$ ee. 


\section{$(R, E)-1-m e t h o x y-2-(1-p h e n y l p e n t a-1,4-d i e n-3-y l) b e n z e n e(3 b)$}<smiles>C=CC(C)(C)c1ccccc1OCc1ccccc1</smiles>

The corresponding compound was prepared following the general procedure by using 1-(2methoxyphenyl)prop-2-en-1-ol $\mathbf{1 b}$, potassium (E)-styryltrifluoroborate $\mathbf{2 a}$ (1.1 equiv), and HF (1.1 equiv). The product was isolated by flash chromatography (hexane/EtOAc=97/3) to afford the product as a colorless oil (54.1 mg, 86\%); ${ }^{1} \mathrm{H}-\mathrm{NMR}\left(400 \mathrm{MHz}, \mathrm{CDCl}_{3}\right): \delta=7.41-7.36(\mathrm{~m}, 2 \mathrm{H}), 7.33-7.27(\mathrm{~m}, 2 \mathrm{H}), 7.27-7.18(\mathrm{~m}, 3 \mathrm{H})$, $6.97(\mathrm{td}, J=7.4,1.2 \mathrm{~Hz}, 1 \mathrm{H}), 6.91(\mathrm{dd}, J=8.7,1.1 \mathrm{~Hz}, 1 \mathrm{H}), 6.47-6.43(\mathrm{~m}, 2 \mathrm{H}), 6.16(\mathrm{ddd}, J=16.9,10.2,6.3 \mathrm{~Hz}$, $1 \mathrm{H}), 5.18(\mathrm{dt}, J=10.2,1.6 \mathrm{~Hz}, 1 \mathrm{H}), 5.14(\mathrm{dt}, J=17.2,1.6 \mathrm{~Hz}, 1 \mathrm{H}), 4.73-4.68(\mathrm{~m}, 1 \mathrm{H}), 3.85(\mathrm{~s}, 3 \mathrm{H}) ;{ }^{13} \mathrm{C}-\mathrm{NMR}(100$ $\left.\mathrm{MHz}, \mathrm{CDCl}_{3}\right): \delta=156.8,139.9,137.6,131.7,130.9,130.3,128.7,128.4,127.5,127.0,126.2,120.6,115.2,110.8$, 55.5, 44.9; IR (neat): 3059, 3025, 3004, 2957, 2936, 2835, 1633, 1598, 1490, 1462, 1437, 1288, 1241, 1105, 1051, 1029, 966, 915, 803, 748, 692, $670 \mathrm{~cm}^{-1}$; HRMS (EI): $\mathrm{m} / \mathrm{z}$ calcd for $\mathrm{C}_{18} \mathrm{H}_{18} \mathrm{O}[\mathrm{M}]^{+} 250.1358$, found 250.1353; SFC: Daicel Chiracel OJ-H, $5 \% \mathrm{MeOH}, 2.0 \mathrm{~mL} / \mathrm{min}, 25^{\circ} \mathrm{C}, 200 \mathrm{~nm}$; $97.5 \%$ ee $\left(t_{R}\right.$ (major) $=9.3 \mathrm{~min}, \mathrm{t}_{\mathrm{R}}($ minor $)=$ $11.4 \mathrm{~min}) ;[\alpha]_{\mathrm{D}}^{24}=+48.1\left(\mathrm{c}=2.0, \mathrm{CHCl}_{3}\right)$.

\section{$(R, E)$-1-methoxy-3-(1-phenylpenta-1,4-dien-3-yl)benzene (3c)}<smiles>C=CC(C=Cc1ccccc1)c1cccc(OC)c1</smiles>

The corresponding compound was prepared following the general procedure by using 1-(3-

methoxyphenyl)prop-2-en-1-ol $\mathbf{1 c}$ and potassium $(E)$-styryltrifluoroborate $\mathbf{2 a}$. The product was isolated by flash chromatography (hexane/EtOAc=97/3) to afford the product as a colorless oil (50.5 mg, 81\%); ${ }^{1} \mathrm{H}$-NMR (400 $\left.\mathrm{MHz}, \mathrm{CDCl}_{3}\right): \delta=7.43-7.37(\mathrm{~m}, 2 \mathrm{H}), 7.34-7.25(\mathrm{~m}, 3 \mathrm{H}), 7.25-7.19(\mathrm{~m}, 1 \mathrm{H}), 6.90(\mathrm{~m}, 1 \mathrm{H}), 6.85(\mathrm{~m}, 1 \mathrm{H}), 6.80$ (ddd, $J=8.2,2.6,1.0 \mathrm{~Hz}, 1 \mathrm{H}), 6.51-6.37(\mathrm{~m}, 2 \mathrm{H}), 6.13$ (ddd, $J=17.1,10.2,6.7 \mathrm{~Hz}, 1 \mathrm{H}), 5.25-5.13(\mathrm{~m}, 2 \mathrm{H}), 4.22$ $(\mathrm{t}, J=6.7 \mathrm{~Hz}, 1 \mathrm{H}), 3.81(\mathrm{~s}, 3 \mathrm{H}) ;{ }^{13} \mathrm{C}-\mathrm{NMR}\left(100 \mathrm{MHz}, \mathrm{CDCl}_{3}\right): \delta=159.8,144.3,139.9,137.3,131.5,130.7,129.5$, 128.5, 127.2, 126.2, 120.4, 115.7, 113.9, 111.7, 55.1, 52.4; IR (neat): 3081, 3057, 3025, 3002, 2956, 2834, 1634, 1597, 1583, 1486, 1449, 1435, 1259, 1149, 1047, 995, 966, 917, 874, 762, 743, $692 \mathrm{~cm}^{-1}$; HRMS (EI): $\mathrm{m} / \mathrm{z}$ calcd for $\mathrm{C}_{18} \mathrm{H}_{18} \mathrm{O}[\mathrm{M}]^{+}$250.1358, found 250.1353; SFC: Daicel Chiracel OJ-H, $5 \% \mathrm{MeOH}, 2.0 \mathrm{~mL} / \mathrm{min}, 25^{\circ} \mathrm{C}, 200 \mathrm{~nm}$; $>99 \%$ ee $\left(t_{R}(\right.$ major $)=13.0 \mathrm{~min}, \mathrm{t}_{\mathrm{R}}($ minor $\left.)=15.1 \mathrm{~min}\right) ;[\alpha]_{\mathrm{D}}^{27}=-11.8\left(\mathrm{c}=2.0, \mathrm{CHCl}_{3}\right)$.

\section{$(R, E)-1-b r o m o-4-(1-p h e n y l p e n t a-1,4-d i e n-3-y l) b e n z e n e ~(3 d)$}<smiles>C=CC(C=Cc1ccccc1)c1ccc(Br)cc1</smiles>

The corresponding compound was prepared following the general procedure by using 1-(4-bromophenyl)prop2-en-1-ol $\mathbf{1 d}$ and potassium $(E)$-styryltrifluoroborate $\mathbf{2} \mathbf{a}$. The product was isolated by flash chromatography 
(hexane/EtOAc=99/1) to afford the product as a colorless oil $(58.5 \mathrm{mg}, 78 \%) ;{ }^{1} \mathrm{H}-\mathrm{NMR}\left(400 \mathrm{MHz}, \mathrm{CDCl}_{3}\right): \delta=$ $7.50-7.43(\mathrm{~m}, 2 \mathrm{H}), 7.41-7.35(\mathrm{~m}, 2 \mathrm{H}), 7.35-7.28(\mathrm{~m}, 2 \mathrm{H}), 7.26-7.20(\mathrm{~m}, 1 \mathrm{H}), 7.18-7.12(\mathrm{~m}, 2 \mathrm{H}), 6.43(\mathrm{~d}, J$ $=16.0 \mathrm{~Hz}, 1 \mathrm{H}), 6.35(\mathrm{dd}, J=16.0,6.7 \mathrm{~Hz}, 1 \mathrm{H}), 6.07(\mathrm{ddd}, J=17.1,10.2,6.7 \mathrm{~Hz}, 1 \mathrm{H}), 5.21(\mathrm{dt}, J=10.2,1.3 \mathrm{~Hz}, 1 \mathrm{H})$, $5.14(\mathrm{dt}, J=17.1,1.3 \mathrm{~Hz}, 1 \mathrm{H}), 4.19(\mathrm{t}, J=6.7 \mathrm{~Hz}, 1 \mathrm{H}) ;{ }^{13} \mathrm{C}-\mathrm{NMR}\left(100 \mathrm{MHz}, \mathrm{CDCl}_{3}\right): \delta=141.5,139.4,137.1,131.6$, 131.0, 131.0, 129.8, 128.5, 127.4, 126.2, 120.4, 116.1, 51.7; IR (neat): 3081, 3059, 3025, 2959, 2922, 2854, 1634, $1598,1485,1448,1401,1259,1216,1180,1101,1072,1010,966,918,870,822,792,746,691 \mathrm{~cm}^{-1}$; HRMS (EI): $\mathrm{m} / \mathrm{z}$ calcd for $\mathrm{C}_{17} \mathrm{H}_{15} \mathrm{Br}[\mathrm{M}]^{+} 298.0357$, found 298.0352; SFC: Daicel Chiracel OJ-H, 2\% MeOH, $2.0 \mathrm{~mL} / \mathrm{min}, 25^{\circ} \mathrm{C}$, $200 \mathrm{~nm} ; 98 \%$ ee $\left(t_{R}\right.$ (major) $=35.5 \mathrm{~min}, \mathrm{t}_{\mathrm{R}}($ minor $\left.)=33.2 \mathrm{~min}\right) ;[\alpha]_{\mathrm{D}}^{26}=-0.68\left(\mathrm{c}=2.0, \mathrm{CHCl}_{3}\right)$.

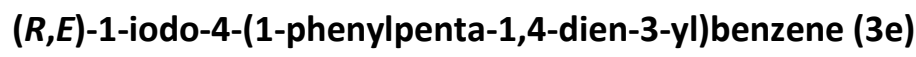

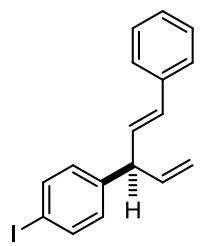

The corresponding compound was prepared following the general procedure by using 1-(4-iodophenyl)prop-2en-1-ol 1e and potassium (E)-styryltrifluoroborate $2 \mathrm{a}$. The product was isolated by flash chromatography (hexane/EtOAc=99/1) to afford the product as a colorless oil $(62.4 \mathrm{mg}, 72 \%) ;{ }^{1} \mathrm{H}-\mathrm{NMR}\left(400 \mathrm{MHz}, \mathrm{CDCl}_{3}\right): \delta=$ $7.70-7.62(\mathrm{~m}, 2 \mathrm{H}), 7.40-7.34(\mathrm{~m}, 2 \mathrm{H}), 7.34-7.26(\mathrm{~m}, 2 \mathrm{H}), 7.25-7.19(\mathrm{~m}, 1 \mathrm{H}), 7.05-6.99(\mathrm{~m}, 2 \mathrm{H}), 6.43(\mathrm{~d}, J$ $=16.0 \mathrm{~Hz}, 1 \mathrm{H}), 6.34(\mathrm{dd}, J=16.0,6.7 \mathrm{~Hz}, 1 \mathrm{H}), 6.06(\mathrm{ddd}, J=17.1,10.2,6.7 \mathrm{~Hz}, 1 \mathrm{H}), 5.21(\mathrm{dt}, J=10.2,1.4 \mathrm{~Hz}, 1 \mathrm{H})$, $5.14(\mathrm{dt}, J=17.1,1.4 \mathrm{~Hz}, 1 \mathrm{H}), 4.17(\mathrm{t}, J=6.7 \mathrm{~Hz}, 1 \mathrm{H}) ;{ }^{13} \mathrm{C}-\mathrm{NMR}\left(100 \mathrm{MHz}, \mathrm{CDCl}_{3}\right): \delta=142.2,139.4,137.5,137.0$, 131.1, 130.9, 130.2, 128.5, 127.4, 126.2, 116.1, 91.8, 51.8; IR (neat):3079, 3057, 3024, 2968, 2923, 2852, 1634, $1598,1481,1447,1397,1261,1251,1183,1101,1061,1005,965,917,872,819,789,745,722,691,625 \mathrm{~cm}^{-1}$; HRMS (EI): $\mathrm{m} / \mathrm{z}$ calcd for $\mathrm{C}_{17} \mathrm{H}_{15} \mathrm{l}[\mathrm{M}]^{+} 346.0218$, found 346.0213; SFC: Daicel Chiracel OJ-H, 10\% MeOH, 3.0 $\mathrm{mL} / \mathrm{min}, 25^{\circ} \mathrm{C}, 200 \mathrm{~nm}$; $99 \%$ ee $\left(\mathrm{t}_{\mathrm{R}}(\right.$ major $\left.)=14.7 \mathrm{~min}, \mathrm{t}_{\mathrm{R}}(\operatorname{minor})=13.6 \mathrm{~min}\right) ;[\alpha]^{26}{ }_{\mathrm{D}}=+2.6\left(\mathrm{c}=2.0, \mathrm{CHCl}_{3}\right)$.

\section{$(R, E)$-methyl 4-(1-phenylpenta-1,4-dien-3-yl)benzoate (3f)}<smiles>C=CC(=CCc1ccccc1)c1ccc(C(C)=O)cc1</smiles>

The corresponding compound was prepared following the general procedure by using methyl 4-(1hydroxyallyl)benzoate $\mathbf{1 f}$ and potassium $(E)$-styryltrifluoroborate $\mathbf{2 a}$. The product was isolated by flash chromatography (hexane/EtOAc=97/3) to afford the product as a colorless oil $(47.1 \mathrm{mg}, 68 \%) ;{ }^{1} \mathrm{H}-\mathrm{NMR}$ (300 $\left.\mathrm{MHz}, \mathrm{CDCl}_{3}\right): \delta=8.06-7.98(\mathrm{~m}, 2 \mathrm{H}), 7.43-7.19(\mathrm{~m}, 7 \mathrm{H}), 6.45(\mathrm{~d}, J=15.9 \mathrm{~Hz}, 1 \mathrm{H}), 6.37(\mathrm{dd}, J=15.9,6.6 \mathrm{~Hz}, 1 \mathrm{H})$, 6.11 (ddd, $J=17.1,10.2,6.6 \mathrm{~Hz}, 1 \mathrm{H}), 5.24(\mathrm{dt}, J=10.2,1.4 \mathrm{~Hz}, 1 \mathrm{H}), 5.17(\mathrm{dt}, J=17.1,1.4 \mathrm{~Hz}, 1 \mathrm{H}), 4.29(\mathrm{t}, J=6.6$ $\mathrm{Hz}, 1 \mathrm{H}), 3.92(\mathrm{~s}, 3 \mathrm{H}) ;{ }^{13} \mathrm{C}-\mathrm{NMR}\left(75 \mathrm{MHz}, \mathrm{CDCl}_{3}\right): \delta=166.9,147.9,139.2,137.0,131.2,130.7,129.9,128.5,128.5$, 128.1, 127.4, 126.2, 116.3, 52.3, 52.0; IR (neat): 3026, 2952, 2924, 2853, 1719, 1608, 1495, 1435, 1413, 1276, $1216,1179,1104,1019,966,920,853,828,747,709,693,667 \mathrm{~cm}^{-1} ; \mathrm{HRMS}(\mathrm{ESI}+): \mathrm{m} / \mathrm{z}$ calcd for $\mathrm{C}_{19} \mathrm{H}_{19} \mathrm{O}_{2}$ $[\mathrm{M}+\mathrm{H}]^{+}$279.1380, found 279.1379; SFC: Daicel Chirapak IA, 5\% MeOH, $2.0 \mathrm{~mL} / \mathrm{min}, 25^{\circ} \mathrm{C}, 200 \mathrm{~nm} ;>99 \%$ ee $\left(\mathrm{t}_{\mathrm{R}}\right.$ $($ major $\left.)=13.0 \mathrm{~min}, \mathrm{t}_{\mathrm{R}}(\operatorname{minor})=11.1 \mathrm{~min}\right) ;[\alpha]_{\mathrm{D}}^{26}=-0.74\left(\mathrm{c}=2.0, \mathrm{CHCl}_{3}\right)$. 


\section{$(R, E)-4-(1-p h e n y l p e n t a-1,4-d i e n-3-y l)$ benzaldehyde (3g)}<smiles>C=CC(C=Cc1ccccc1)c1ccc(C=O)cc1</smiles>

The corresponding compound was prepared following the general procedure using 4-(1hydroxyallyl)benzaldehyde $\mathbf{1} \mathbf{g}$ and potassium $(E)$-styryltrifluoroborate $\mathbf{2 a}$. The product was isolated by flash chromatography (hexane/EtOAc=97/3) to afford the product as a colorless oil $(40.2 \mathrm{mg}, 65 \%) ;{ }^{1} \mathrm{H}-\mathrm{NMR}$ (400 $\left.\mathrm{MHz}, \mathrm{CDCl}_{3}\right): \delta=10.01(\mathrm{~s}, 1 \mathrm{H}), 7.90-7.84(\mathrm{~m}, 2 \mathrm{H}), 7.49-7.43(\mathrm{~m}, 2 \mathrm{H}), 7.41-7.36(\mathrm{~m}, 2 \mathrm{H}), 7.36-7.28(\mathrm{~m}, 2 \mathrm{H})$, $7.28-7.21(\mathrm{~m}, 1 \mathrm{H}), 6.50(\mathrm{~d}, J=15.9 \mathrm{~Hz}, 1 \mathrm{H}), 6.41(\mathrm{dd}, J=15.9,6.7 \mathrm{~Hz}, 1 \mathrm{H}), 6.12(\mathrm{ddd}, J=17.1,10.2,6.7 \mathrm{~Hz}, 1 \mathrm{H}$ ), $5.26(\mathrm{dt}, J=10.2,1.4 \mathrm{~Hz}, 1 \mathrm{H}), 5.18(\mathrm{dt}, J=17.1,1.4 \mathrm{~Hz}, 1 \mathrm{H}), 4.32(\mathrm{t}, J=6.7 \mathrm{~Hz}, 1 \mathrm{H}) ;{ }^{13} \mathrm{C}-\mathrm{NMR}\left(100 \mathrm{MHz}, \mathrm{CDCl}_{3}\right): \delta$ $=191.9,149.7,138.9,136.9,135.0,131.5,130.4,130.1,128.7,128.5,127.5,126.3,116.6,52.4$; IR (neat): 3024, 2962, 2926, 2849, 2732, 1698, 1604, 1575, 1495, 1448, 1414, 1389, 1304, 1260, 1209, 1167, 1092, 1016, 967, 920, 822, 794, 746, 692, $666 \mathrm{~cm}^{-1}$; HRMS (EI): $\mathrm{m} / z$ calcd for $\mathrm{C}_{18} \mathrm{H}_{16} \mathrm{O}[\mathrm{M}]^{+}$248.1201, found 248.1196; SFC: Daicel Chirapak IA, $10 \% \mathrm{MeOH}, 2.0 \mathrm{~mL} / \mathrm{min}, 25^{\circ} \mathrm{C}, 200 \mathrm{~nm}$; $99 \%$ ee $\left(t_{R}(\right.$ major $)=9.4 \mathrm{~min}, \mathrm{t}_{\mathrm{R}}($ minor $\left.)=8.0 \mathrm{~min}\right) ;[\alpha]_{\mathrm{D}}^{26}=$ $+2.6\left(\mathrm{c}=2.0, \mathrm{CHCl}_{3}\right)$.

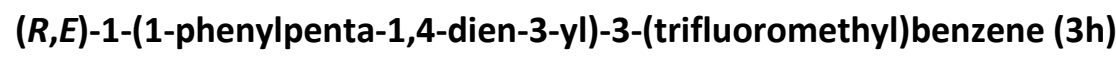<smiles>C=CC(/C=C/c1ccccc1)c1cccc(C(F)(F)F)c1</smiles>

The corresponding compound was prepared following the general procedure by using 1-(3-

(trifluoromethyl)phenyl)prop-2-en-1-ol $\mathbf{1}$ h and potassium (E)-styryltrifluoroborate $\mathbf{2 a}$. The product was isolated by flash chromatography (hexane/EtOAc=99/1) to afford the product as a colorless oil $(44.0 \mathrm{mg}, 61 \%) ;{ }^{1} \mathrm{H}-\mathrm{NMR}$ $\left(400 \mathrm{MHz}, \mathrm{CDCl}_{3}\right): \delta=7.55-7.41(\mathrm{~m}, 4 \mathrm{H}), 7.41-7.36(\mathrm{~m}, 2 \mathrm{H}), 7.35-7.28(\mathrm{~m}, 2 \mathrm{H}), 7.27-7.20(\mathrm{~m}, 1 \mathrm{H}), 6.47(\mathrm{~d}$, $J=16.0 \mathrm{~Hz}, 1 \mathrm{H}), 6.37(\mathrm{dd}, J=16.0,6.7 \mathrm{~Hz}, 1 \mathrm{H}), 6.10(\mathrm{ddd}, J=17.1,10.2,6.7 \mathrm{~Hz}, 1 \mathrm{H}), 5.25(\mathrm{dt}, J=10.2,1.3 \mathrm{~Hz}$, $1 \mathrm{H}), 5.17(\mathrm{dt}, J=17.1,1.3 \mathrm{~Hz}, 1 \mathrm{H}), 4.29(\mathrm{t}, J=6.7 \mathrm{~Hz}, 1 \mathrm{H}) ;{ }^{13} \mathrm{C}-\mathrm{NMR}\left(100 \mathrm{MHz}, \mathrm{CDCl}_{3}\right): \delta=143.6,139.1,137.0$, $131.5(q, J=1.4 \mathrm{~Hz}), 131.4,130.9(\mathrm{q}, J=32.1 \mathrm{~Hz}), 130.6,129.0,128.6,127.5,126.3,124.8(\mathrm{q}, J=3.8 \mathrm{~Hz}), 124.2$ $(q, J=272.3 \mathrm{~Hz}) 123.5$ (q, $J=3.8 \mathrm{~Hz}), 116.5,52.1 ;{ }^{19} \mathrm{~F}-\mathrm{NMR}\left(376 \mathrm{MHz}, \mathrm{CDCl}_{3}\right): \delta=-62.4$; IR (neat): 3085, 3028, 2980, 2929, 1636, 1597, 1496, 1448, 1327, 1162, 1121, 1096, 1073, 994, 967, 920, 801, 760, 744, $691 \mathrm{~cm}^{-1}$; HRMS (EI): $\mathrm{m} / \mathrm{z}$ calcd for $\mathrm{C}_{18} \mathrm{H}_{15} \mathrm{~F}_{3}$ [M] ${ }^{+}$288.1126, found 288.1121; SFC: Daicel Chiracel OJ-H, $100 \% \mathrm{CO}_{2}, 3.0$ $\mathrm{mL} / \mathrm{min}, 25^{\circ} \mathrm{C}, 200 \mathrm{~nm} ;>99 \%$ ee $\left(\mathrm{t}_{\mathrm{R}}\right.$ (major) $=7.9 \mathrm{~min}, \mathrm{t}_{\mathrm{R}}($ minor $\left.)=8.3 \mathrm{~min}\right) ;[\alpha]^{26}{ }_{\mathrm{D}}=-11.9\left(\mathrm{c}=2.0, \mathrm{CHCl}_{3}\right)$. 


\section{(R,E)-1-nitro-4-(1-phenylpenta-1,4-dien-3-yl)benzene (3i)}<smiles>C=CC(C=Cc1ccccc1)c1ccc([N+](=O)[O-])cc1</smiles>

The corresponding compound was prepared following the general procedure by using 1-(4-nitrophenyl)prop-2en-1-ol $\mathbf{1} \mathbf{i}$ and potassium $(E)$-styryltrifluoroborate $\mathbf{2} \mathbf{a}$. The product was isolated by flash chromatography (hexane/EtOAc=98/2) to afford the product as a pale yellow oil $(36.3 \mathrm{mg}, 55 \%) ;{ }^{1} \mathrm{H}-\mathrm{NMR}\left(400 \mathrm{MHz}, \mathrm{CDCl}_{3}\right): \delta=$ $8.24-8.17(\mathrm{~m}, 2 \mathrm{H}), 7.49-7.42(\mathrm{~m}, 2 \mathrm{H}), 7.41-7.37(\mathrm{~m}, 2 \mathrm{H}), 7.36-7.30(\mathrm{~m}, 2 \mathrm{H}), 7.29-7.22(\mathrm{~m}, 1 \mathrm{H}), 6.48(\mathrm{~d}, J$ $=16.0 \mathrm{~Hz}, 1 \mathrm{H}$ ), $6.36(\mathrm{dd}, J=16.0,6.8 \mathrm{~Hz}, 1 \mathrm{H}), 6.10(\mathrm{ddd}, J=17.1,10.3,6.8 \mathrm{~Hz}, 1 \mathrm{H}), 5.29(\mathrm{dt}, J=10.3,1.3 \mathrm{~Hz}, 1 \mathrm{H}$ ), $5.19(\mathrm{dt}, J=17.1,1.3 \mathrm{~Hz}, 1 \mathrm{H}), 4.35(\mathrm{t}, J=6.8 \mathrm{~Hz}, 1 \mathrm{H}) ;{ }^{13} \mathrm{C}-\mathrm{NMR}\left(100 \mathrm{MHz}, \mathrm{CDCl}_{3}\right): \delta=150.2,146.7,138.5,136.7$, 131.9, 129.8, 128.9, 128.6, 127.7, 126.3, 123.8, 117.0, 52.0; IR (neat): 3081, 3026, 2960, 2923, 2853, 1597, 1515, 1492, 1448, 1342, 1262, 1109, 1014, 967, 922, 852, 799, 744, $692 \mathrm{~cm}^{-1}$; HRMS (EI): $\mathrm{m} / \mathrm{z}$ calcd for $\mathrm{C}_{17} \mathrm{H}_{15} \mathrm{NO}_{2}[\mathrm{M}]^{+}$ 265.1103, found 265.1098; SFC: Daicel Chiracel OJ-H, $5 \% \mathrm{MeOH}, 2.0 \mathrm{~mL} / \mathrm{min}, 25^{\circ} \mathrm{C}, 200 \mathrm{~nm}$; $>99 \%$ ee ( $\mathrm{t}_{\mathrm{R}}$ (major) $=37.5 \mathrm{~min}, \mathrm{t}_{\mathrm{R}}($ minor $\left.)=34.3 \mathrm{~min}\right) ;[\alpha]^{26}=-0.14^{\circ}\left(\mathrm{c}=2.0, \mathrm{CHCl}_{3}\right)$.

\section{(R,E)-2-(1-phenylpenta-1,4-dien-3-yl)naphthalene (3j)}<smiles>C=CC(C=Cc1ccccc1)c1ccc2ccccc2c1</smiles>

The corresponding compound was prepared following the general procedure by using 1-(naphthalen-2-yl)prop2-en-1-ol $\mathbf{1 j}$ and potassium (E)-styryltrifluoroborate $\mathbf{2 a}$. The product was isolated by flash chromatography (hexane/EtOAc=99/1) to afford the product as an amorphous white solid $(61.0 \mathrm{mg}, 90 \%) ;{ }^{1} \mathrm{H}-\mathrm{NMR}(400 \mathrm{MHz}$, $\left.\mathrm{CDCl}_{3}\right): \delta=7.87-7.79(\mathrm{~m}, 3 \mathrm{H}), 7.73(\mathrm{~s}, 1 \mathrm{H}), 7.51-7.38(\mathrm{~m}, 5 \mathrm{H}), 7.35-7.28(\mathrm{~m}, 2 \mathrm{H}), 7.26-7.20(\mathrm{~m}, 1 \mathrm{H}), 6.56-$ $6.47(\mathrm{~m}, 2 \mathrm{H}), 6.23(\mathrm{ddd}, J=17.1,10.2,6.7 \mathrm{~Hz}, 1 \mathrm{H}), 5.27(\mathrm{dt}, J=10.2,1.5 \mathrm{~Hz}, 1 \mathrm{H}), 5.22(\mathrm{dt}, J=17.1,1.5 \mathrm{~Hz}, 1 \mathrm{H})$, $4.44-4.38(\mathrm{~m}, 1 \mathrm{H}) ;{ }^{13} \mathrm{C}-\mathrm{NMR}\left(100 \mathrm{MHz}, \mathrm{CDCl}_{3}\right): \delta=140.0,139.9,137.3,133.6,132.3,131.6,130.9,128.5,128.1$, 127.7, 127.6, 127.3, 126.8, 126.3, 126.3, 126.0, 125.5, 115.9, 52.4; IR (neat): 3056, 3024, 2962, 2925, 2856, 2360, 2338, 1631, 1598, 1495, 1447, 1128, 1071, 1019, 997, 968, 912, 856, 817, 777, 762, 737, 690, $666 \mathrm{~cm}^{-1}$; HRMS (EI): $\mathrm{m} / \mathrm{z}$ calcd for $\mathrm{C}_{21} \mathrm{H}_{18}[\mathrm{M}]^{+} 270.1409$, found 270.1407; SFC: Daicel Chiracel OJ-H, 10\% MeOH, 2.0 $\mathrm{mL} / \mathrm{min}, 25^{\circ} \mathrm{C}, 200 \mathrm{~nm} ; 98 \%$ ee $\left(\mathrm{t}_{\mathrm{R}}\right.$ (major) $=35.0 \mathrm{~min}, \mathrm{t}_{\mathrm{R}}($ minor $\left.)=38.6 \mathrm{~min}\right) ;[\alpha]_{\mathrm{D}}^{26}=+0.69\left(\mathrm{c}=2.0, \mathrm{CHCl}_{3}\right)$.

\section{$(R, E)$-3-(1-phenylpenta-1,4-dien-3-yl)thiophene (3k)}

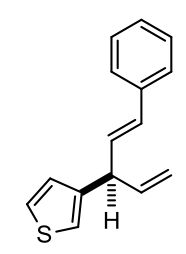

The corresponding compound was prepared following the general procedure by using 1-(thiophen-3-yl)prop-2en-1-ol $\mathbf{1} \mathbf{k}$, potassium ( $E$ )-styryltrifluoroborate $\mathbf{2 a}$ (1.1 equiv), and HF (1.1 equiv). The product was isolated by flash chromatography (hexane/EtOAc=99/1) to afford the product as a colorless oil $(47.7 \mathrm{mg}, 84 \%) ;{ }^{1} \mathrm{H}-\mathrm{NMR}$ 
$\left(400 \mathrm{MHz}, \mathrm{CDCl}_{3}\right): \delta=7.42-7.35(\mathrm{~m}, 2 \mathrm{H}), 7.34-7.28(\mathrm{~m}, 3 \mathrm{H}), 7.25-7.18(\mathrm{~m}, 1 \mathrm{H}), 7.05(\mathrm{dt}, J=3.0,1.1 \mathrm{~Hz}, 1 \mathrm{H})$, $7.00(\mathrm{dd}, J=5.0,1.1 \mathrm{~Hz}, 1 \mathrm{H}), 6.45(\mathrm{~d}, J=15.9 \mathrm{~Hz}, 1 \mathrm{H}), 6.38(\mathrm{dd}, J=15.9,7.0 \mathrm{~Hz}, 1 \mathrm{H}), 6.10(\mathrm{ddd}, J=15.5,10.2,7.0$ $\mathrm{Hz}, 1 \mathrm{H}), 5.19(\mathrm{dt}, J=7.0,1.5 \mathrm{~Hz}, 1 \mathrm{H}), 5.15(\mathrm{dt}, J=15.5,1.5 \mathrm{~Hz}, 1 \mathrm{H}), 4.30(\mathrm{t}, J=7.0 \mathrm{~Hz}, 1 \mathrm{H}) ;{ }^{13} \mathrm{C}-\mathrm{NMR}(100 \mathrm{MHz}$, $\left.\mathrm{CDCl}_{3}\right): \delta=143.2,139.50,137.3,131.1,130.5,128.5,127.7,127.3,126.2,125.6,120.8,115.5,48.0$; IR (neat): $3080,3059,3025,2923,2853,1871,1634,1598,1577,1495,1448,1411,1306,1262,1154,1079,1028,993$, $964,917,850,782,752,731,691 \mathrm{~cm}^{-1}$; HRMS (EI): $\mathrm{m} / z$ calcd for $\mathrm{C}_{15} \mathrm{H}_{14} \mathrm{~S}[\mathrm{M}]^{+} 226.0816$, found 226.0812; SFC: Daicel Chiracel $\mathrm{OJ}-\mathrm{H}, 10 \% \mathrm{MeOH}, 2.0 \mathrm{~mL} / \mathrm{min}, 25^{\circ} \mathrm{C}, 200 \mathrm{~nm} ; 97 \%$ ee $\left(\mathrm{t}_{\mathrm{R}}\right.$ (major) $=11.0 \mathrm{~min}, \mathrm{t}_{\mathrm{R}}(\operatorname{minor})=11.7$ $\min ) ;[\alpha]_{D}^{26}=-23.9\left(\mathrm{c}=2.0, \mathrm{CHCl}_{3}\right)$.

\section{$(R, E)$-tert-butyl 3-(1-phenylpenta-1,4-dien-3-yl)-1H-indole-1-carboxylate (3I)}<smiles>C=CC(C=Cc1ccccc1)C=Cc1ccccc1[O+]</smiles>

The corresponding compound was prepared following the general procedure by using tert-butyl 3-(1hydroxyallyl)-1H-indole-1-carboxylate $1 \mathrm{l}$, potassium ( $E$ )-styryltrifluoroborate $2 \mathrm{2a}$ (1.1 equiv), and HF (1.1 equiv). The reaction mixture was purified via flash chromatography (hexane/EtOAc=97:3) to afford the mixture of $\mathbf{3}$ | and the linear regioisomer as a yellow oil ( $89.1 \mathrm{mg}, 99 \%, 3: 1=$ branched:linear). The regioisomers were separated via $\mathrm{AgNO}_{3}$ impregnated silica preparative TLC (benzene) to afford $3 \mathbf{I}$ as a yellow oil for characterization; ${ }^{1} \mathrm{H}-\mathrm{NMR}\left(400 \mathrm{MHz}, \mathrm{CDCl}_{3}\right): \delta=8.16(\mathrm{~d}, \mathrm{~J}=1.3 \mathrm{~Hz}, 1 \mathrm{H}), 7.62-7.57(\mathrm{~m}, 1 \mathrm{H}), 7.44(\mathrm{br}, 1 \mathrm{H}), 7.41-$ $7.36(\mathrm{~m}, 2 \mathrm{H}), 7.36-7.27(\mathrm{~m}, 3 \mathrm{H}), 7.25-7.19(\mathrm{~m}, 2 \mathrm{H}), 6.53(\mathrm{~d}, J=15.9 \mathrm{~Hz}, 1 \mathrm{H}), 6.47(\mathrm{dd}, J=15.9,6.3 \mathrm{~Hz}, 1 \mathrm{H})$, $6.24-6.14(\mathrm{~m}, 1 \mathrm{H}), 5.26(\mathrm{dt}, J=6.3,1.4 \mathrm{~Hz}, 1 \mathrm{H}), 5.23(\mathrm{~d}, J=1.4 \mathrm{~Hz}, 1 \mathrm{H}), 4.45(\mathrm{t}, J=6.3 \mathrm{~Hz}, 1 \mathrm{H}), 1.68(\mathrm{~s}, 9 \mathrm{H}) ;{ }^{13} \mathrm{C}-$ $\operatorname{NMR}\left(100 \mathrm{MHz}, \mathrm{CDCl}_{3}\right): \delta=149.8,138.6,137.2,135.8,131.0,130.3,129.7,128.5,127.3,126.3,124.3,122.8$, $122.3,121.7,119.9,116.0,115.3,83.5,43.6,28.2$; IR (neat): 3024, 2979, 2932, 1728, 1604, 1476, 1451, 1369, $1308,1255,1215,1152,1079,1017,967,920,853,743,692,667 \mathrm{~cm}^{-1} ; \mathrm{HRMS}(\mathrm{ESI}+): \mathrm{m} / z$ calcd for $\mathrm{C}_{24} \mathrm{H}_{25} \mathrm{NO}_{2}$ $[\mathrm{M}+\mathrm{Na}]^{+} 382.1778$, found 382.1778; SFC: Daicel Chirapak IB, 5\% MeOH, $2.0 \mathrm{~mL} / \mathrm{min}, 25^{\circ} \mathrm{C}, 200 \mathrm{~nm}$; $89 \%$ ee $\left(\mathrm{t}_{\mathrm{R}}\right.$ (major) $=6.5 \mathrm{~min}, \mathrm{t}_{\mathrm{R}}$ (minor $\left.)=7.0 \mathrm{~min}\right) ;[\alpha]_{\mathrm{D}}^{25}=-12.6\left(\mathrm{c}=2.0, \mathrm{CHCl}_{3}\right)$.

\section{$(R, E)$-1-fluoro-4-(3-phenylpenta-1,4-dien-1-yl)benzene (3m)}

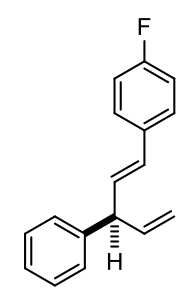

The corresponding compound was prepared following the general procedure by using 1-phenylprop-2-en-1-ol $1 \mathrm{a}$ and potassium $(E)$-(4-fluorostyryl)trifluoroborate $\mathbf{2 m}$. The product was isolated by flash chromatography (hexane/EtOAc=99/1) to afford the product as a colorless oil $(45.0 \mathrm{mg}, 76 \%) ;{ }^{1} \mathrm{H}-\mathrm{NMR}(400 \mathrm{MHz}, \mathrm{CDCl} 3): \delta=$ $7.40-7.30(\mathrm{~m}, 4 \mathrm{H}), 7.30-7.21(\mathrm{~m}, 3 \mathrm{H}), 7.03-6.95(\mathrm{~m}, 2 \mathrm{H}), 6.41(\mathrm{~d}, J=16.0 \mathrm{~Hz}, 1 \mathrm{H}), 6.32(\mathrm{dd}, J=16.0,6.7 \mathrm{~Hz}$, $1 \mathrm{H}), 6.12$ (ddd, $J=17.2,10.2,6.7 \mathrm{~Hz}, 1 \mathrm{H}), 5.20(\mathrm{dt}, J=10.2,1.4 \mathrm{~Hz}, 1 \mathrm{H}), 5.15(\mathrm{dt}, J=17.2,1.4 \mathrm{~Hz}, 1 \mathrm{H}), 4.22(\mathrm{t}, J=$ 6.7 Hz, 1H); ${ }^{13} \mathrm{C}-\mathrm{NMR}\left(100 \mathrm{MHz}, \mathrm{CDCl}_{3}\right): \delta=162.1(\mathrm{~d}, J=246.3 \mathrm{~Hz}), 142.5,140.0,133.5(\mathrm{~d}, J=3.3 \mathrm{~Hz}), 131.5(\mathrm{~d}, J$ $=2.3 \mathrm{~Hz}), 129.4,128.6,128.0,127.7$ (d, J = 7.9 Hz), 126.6, 115.7, $115.3(\mathrm{~d}, J=21.6 \mathrm{~Hz}), 52.3 ;{ }^{19} \mathrm{~F}-\mathrm{NMR}(376 \mathrm{MHz}$, $\mathrm{CDCl}_{3}$ ): $\delta=-115.0$; IR (neat): 3077, 3027, 2979, 1886, 1635, 1601, 1507, 1451, 1412, 1298, 1225, 1157, 1095, 
995, 967, 918, 852, 833, 804, 754, $699 \mathrm{~cm}^{-1}$; HRMS (EI): $\mathrm{m} / \mathrm{z}$ calcd for $\mathrm{C}_{17} \mathrm{H}_{15} \mathrm{~F}[\mathrm{M}]^{+}$238.1158, found 238.1155; SFC: Daicel Chiracel OJ-H, $2 \% \mathrm{MeOH}, 2.0 \mathrm{~mL} / \mathrm{min}, 25^{\circ} \mathrm{C}, 200 \mathrm{~nm}$; $97 \%$ ee $\left(\mathrm{t}_{\mathrm{R}}\right.$ (major) $=16.7 \mathrm{~min}, \mathrm{t}_{\mathrm{R}}(\operatorname{minor})=$ $22.9 \mathrm{~min}) ;[\alpha]_{\mathrm{D}}^{27}=-10.8\left(\mathrm{c}=2.0, \mathrm{CHCl}_{3}\right)$.

\section{$(R, E)$-1-methoxy-4-(3-phenylpenta-1,4-dien-1-yl)benzene (3n)}<smiles>C=CC(C=Cc1ccc(OC)cc1)c1ccccc1</smiles>

The corresponding compound was prepared following the general procedure by using 1-phenylprop-2-en-1-ol $\mathbf{1 a}$ and potassium (E)-(4-methoxystyryl)trifluoroborate $\mathbf{2} \mathbf{n}$. The product was isolated by flash chromatography (hexane/EtOAc=99/1) to afford the product as a colorless oil $(51.6 \mathrm{mg}, 82 \%) ;{ }^{1} \mathrm{H}-\mathrm{NMR}\left(400 \mathrm{MHz}, \mathrm{CDCl}_{3}\right): \delta=$ $7.37-7.21(\mathrm{~m}, 7 \mathrm{H}), 6.87-6.82(\mathrm{~m}, 2 \mathrm{H}), 6.39(\mathrm{~d}, J=16.0 \mathrm{~Hz}, 1 \mathrm{H}), 6.27(\mathrm{dd}, J=16.0,6.8 \mathrm{~Hz}, 1 \mathrm{H}), 6.12(\mathrm{ddd}, J=$ $17.1,10.3,6.8 \mathrm{~Hz}, 1 \mathrm{H}), 5.19(\mathrm{dt}, J=10.3,1.5 \mathrm{~Hz}, 1 \mathrm{H}), 5.14(\mathrm{dt}, J=17.1,1.5 \mathrm{~Hz}, 1 \mathrm{H}), 4.21(\mathrm{t}, J=6.8 \mathrm{~Hz}, 1 \mathrm{H}), 3.80$ $(\mathrm{s}, 3 \mathrm{H}) ;{ }^{13} \mathrm{C}-\mathrm{NMR}\left(100 \mathrm{MHz}, \mathrm{CDCl}_{3}\right): \delta=158.9,142.8,140.3,130.1,130.0,129.6,128.5,128.0,127.3,126.4$, 115.4, 113.9, 55.3, 52.4; IR (neat): 3026, 3007, 2956, 2934, 2909, 2836, 1635, 1607, 1577, 1510, 1492, 1452, 1294, 1247, 1217, 1174, 1032, 995, 967, 917, 829, 750, 699, $667 \mathrm{~cm}^{-1}$; HRMS (EI): $\mathrm{m} / \mathrm{z}$ calcd for $\mathrm{C}_{18} \mathrm{H}_{18} \mathrm{O}[\mathrm{M}]^{+}$ 250.1358, found 250.1353; SFC: Daicel Chiracel OJ-H, $5 \% \mathrm{MeOH}, 2.0 \mathrm{~mL} / \mathrm{min}, 25^{\circ} \mathrm{C}, 200 \mathrm{~nm}$; $95 \%$ ee ( $\mathrm{t}_{\mathrm{R}}$ (major) $=19.8 \mathrm{~min}, \mathrm{t}_{\mathrm{R}}($ minor $\left.)=26.7 \mathrm{~min}\right) ;[\alpha]_{\mathrm{D}}^{26}=-5.6\left(\mathrm{c}=2.0, \mathrm{CHCl}_{3}\right)$.

\section{(R,Z)-1-methoxy-4-(3-phenylpenta-1,4-dien-1-yl)benzene (30)}<smiles>C=CC(C=Cc1ccc(OC)cc1)(c1ccccc1)c1ccccc1</smiles>

The corresponding compound was prepared following the general procedure by using 1-phenylprop-2-en-1-ol $\mathbf{1 a}$ and potassium (Z)-(4-methoxystyryl)trifluoroborate $\mathbf{2 0}$. The product was isolated by flash chromatography (hexane/benzene $=4 / 1)$ to afford the product as a colorless oil $(43.7 \mathrm{mg}, 70 \%) ;{ }^{1} \mathrm{H}-\mathrm{NMR}\left(400 \mathrm{MHz}, \mathrm{CDCl}_{3}\right): \delta=$ $7.35-7.29(\mathrm{~m}, 2 \mathrm{H}), 7.27-7.19(\mathrm{~m}, 5 \mathrm{H}), 6.91-6.86(\mathrm{~m}, 2 \mathrm{H}), 6.57(\mathrm{~d}, J=11.4 \mathrm{~Hz}, 1 \mathrm{H}), 6.06$ (ddd, $J=17.1,10.1$, $6.1 \mathrm{~Hz}, 1 \mathrm{H}), 5.73(\mathrm{dd}, J=11.4,9.9 \mathrm{~Hz}, 1 \mathrm{H}), 5.21(\mathrm{dt}, J=10.1,1.6 \mathrm{~Hz}, 1 \mathrm{H}), 5.18(\mathrm{~m}, 1 \mathrm{H}), 4.59(\mathrm{dd}, J=9.9,6.1 \mathrm{~Hz}$, $1 \mathrm{H}), 3.82(\mathrm{~s}, 3 \mathrm{H}) ;{ }^{13} \mathrm{C}-\mathrm{NMR}\left(100 \mathrm{MHz}, \mathrm{CDCl}_{3}\right): \delta=158.6,143.4,140.5,131.4,129.8,129.6,128.9,128.6,127.7$, 126.4, 115.2, 113.6, 55.2, 47.7; IR (neat): 3060, 3006, 2954, 2932, 2835, 1607, 1574, 1509, 1492, 1452, 1302, 1246, 1174, 1033, 995, 917, 837, 816, 753, 699, $675 \mathrm{~cm}^{-1}$; HRMS (EI): $\mathrm{m} / z$ calcd for $\mathrm{C}_{18} \mathrm{H}_{18} \mathrm{O}[\mathrm{M}]^{+} 250.1358$, found 250.1355; SFC: Daicel Chiracel OJ-H, $5 \% \mathrm{MeOH}, 2.0 \mathrm{~mL} / \mathrm{min}, 25^{\circ} \mathrm{C}, 200 \mathrm{~nm} ;>99 \%$ ee ( $t_{R}$ (major) $=19.8$ $\left.\min , \mathrm{t}_{\mathrm{R}}(\operatorname{minor})=14.1 \mathrm{~min}\right) ;[\alpha]_{\mathrm{D}}^{27}=-176.9\left(\mathrm{c}=1.0, \mathrm{CHCl}_{3}\right)$. 


\section{(S)-(1-(4,4-dimethylcyclohexa-1,5-dien-1-yl)allyl)benzene (3p)}<smiles>C=CC(c1ccccc1)(c1ccc(C)cc1)C1(C)C=CCC1</smiles>

The corresponding compound was prepared following the general procedure by using 1-phenylprop-2-en-1-ol $\mathbf{1 a}$ and potassium $(E)$-(2-(4,4-dimethylcyclohexa-1,5-dien-1-yl)vinyl)trifluoroborate $\mathbf{2} \mathbf{p}$. The product was isolated by silica plug filtration $\left(\mathrm{CH}_{2} \mathrm{Cl}_{2}\right)$, followed by preparative TLC (hexane/benzene=9/1) to afford the product as a colorless oil $(40.0 \mathrm{mg}, 71 \%) ;{ }^{1} \mathrm{H}-\mathrm{NMR}\left(400 \mathrm{MHz}, \mathrm{CDCl}_{3}\right): \delta=7.35-7.28(\mathrm{~m}, 2 \mathrm{H}), 7.25-7.18(\mathrm{~m}, 3 \mathrm{H})$, $6.13(\mathrm{ddd}, J=17.2,10.3,7.7 \mathrm{~Hz}, 1 \mathrm{H}), 5.82(\mathrm{dd}, J=9.4,5.3 \mathrm{~Hz}, 1 \mathrm{H}), 5.74(\mathrm{dt}, J=5.3,1.3 \mathrm{~Hz}, 1 \mathrm{H}), 5.43(\mathrm{~d}, J=9.4$ $\mathrm{Hz}, 1 \mathrm{H}), 5.16(\mathrm{dt}, J=10.3,1.4 \mathrm{~Hz}, 1 \mathrm{H}), 5.02(\mathrm{dt}, J=17.2,1.4 \mathrm{~Hz}, 1 \mathrm{H}), 4.00(\mathrm{~d}, J=7.7 \mathrm{~Hz}, 1 \mathrm{H}), 1.91(\mathrm{~d}, J=1.3 \mathrm{~Hz}$, $2 \mathrm{H}), 0.92(\mathrm{~s}, 3 \mathrm{H}), 0.89(\mathrm{~s}, 3 \mathrm{H}) ;{ }^{13} \mathrm{C}-\mathrm{NMR}\left(100 \mathrm{MHz}, \mathrm{CDCl}_{3}\right): \delta=141.4,139.2,139.0,136.0,128.5,128.3,126.3$, 121.8, 119.0, 116.0, 56.4, 41.0, 31.7, 27.9, 27.8; IR (neat): 3060, 3026, 2954, 2920, 2864, 1637, 1588, 1492, $1466,1451,1358,1217,1070,1030,996,917,756,726,699,668 \mathrm{~cm}^{-1} ; \mathrm{HRMS}$ (EI): $\mathrm{m} / z$ calcd for $\mathrm{C}_{17} \mathrm{H}_{20}[\mathrm{M}]^{+}$ 224.1565, found 224.1560; SFC: Daicel Chiracel OJ-H, $100 \% \mathrm{CO}_{2}, 2.0 \mathrm{~mL} / \mathrm{min}, 25^{\circ} \mathrm{C}, 200 \mathrm{~nm}$; $97 \%$ ee ( $t_{R}$ (major) $=5.8 \mathrm{~min}, \mathrm{t}_{\mathrm{R}}($ minor $\left.)=6.6 \mathrm{~min}\right) ;[\alpha]_{\mathrm{D}}^{26}=-41.1\left(\mathrm{c}=2.0, \mathrm{CHCl}_{3}\right)$.

\section{$(R, E)-(1-(c y c l o h e x-1-e n-1-y l) p e n t a-1,4-d i e n-3-y l)$ benzene (3q)}

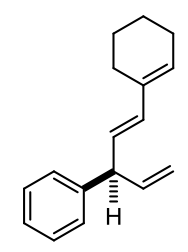

The corresponding compound was prepared following the general procedure by using 1-phenylprop-2-en-1-ol $\mathbf{1 a}$ and potassium (E)-(2-(cyclohex-1-en-1-yl)vinyl)trifluoroborate $\mathbf{2 q}$. The product was isolated by flash chromatography (hexane) to afford the product as a colorless oil $(36.6 \mathrm{mg}, 65 \%) ;{ }^{1} \mathrm{H}-\mathrm{NMR}\left(400 \mathrm{MHz}, \mathrm{CDCl}_{3}\right): \delta=$ $7.35-7.28(\mathrm{~m}, 2 \mathrm{H}), 7.26-7.18(\mathrm{~m}, 3 \mathrm{H}), 6.13-6.00(\mathrm{~m}, 2 \mathrm{H}), 5.78-5.68(\mathrm{~m}, 2 \mathrm{H}), 5.13(\mathrm{dt}, J=10.2,1.6 \mathrm{~Hz}, 1 \mathrm{H})$, $5.09(\mathrm{dt}, J=17.1,1.6 \mathrm{~Hz}, 1 \mathrm{H}), 4.09(\mathrm{t}, J=7.1 \mathrm{~Hz}, 1 \mathrm{H}), 2.19-2.08(\mathrm{~m}, 4 \mathrm{H}), 1.72-1.55(\mathrm{~m}, 4 \mathrm{H}) ;{ }^{13} \mathrm{C}-\mathrm{NMR}(100$ $\left.\mathrm{MHz}_{1} \mathrm{CDCl}_{3}\right): \delta=143.2,140.6,135.4,134.2,128.7,128.4,127.9,127.5,126.3,115.0,52.4,25.8,24.6,22.6,22.5 ;$ IR (neat): 3081, 3061, 3025, 2925, 2857, 2832, 1636, 1599, 1492, 1449, 1240, 1135, 1075, 1029, 995, 964, 914, 839, 790, 755, 698, $667 \mathrm{~cm}^{-1}$; HRMS (EI): $\mathrm{m} / z$ calcd for $\mathrm{C}_{17} \mathrm{H}_{20}[\mathrm{M}]^{+} 224.1565$, found 224.1560; SFC: Daicel Chiracel OJ-H, $2 \% \mathrm{MeOH}, 2.0 \mathrm{~mL} / \mathrm{min}, 25^{\circ} \mathrm{C}, 200 \mathrm{~nm}$; $>99 \%$ ee $\left(\mathrm{t}_{\mathrm{R}}\right.$ (major) = $11.4 \mathrm{~min}, \mathrm{t}_{\mathrm{R}}$ (minor) = $\left.12.7 \mathrm{~min}\right)$; $[\alpha]_{D}^{26}=-39.5\left(\mathrm{c}=2.0, \mathrm{CHCl}_{3}\right)$.

\section{(R)-(1-(cyclopent-1-en-1-yl)allyl)benzene (3r)}<smiles>C=C[C@@H](C1=CCCC1)c1ccccc1</smiles>

The corresponding compound was prepared following the general procedure by using 1-phenylprop-2-en-1-ol $\mathbf{1 a}$ and potassium cyclopent-1-en-1-yltrifluoroborate $\mathbf{2 r}$. The reaction mixture was purified via flash chromatography (hexane) to afford the mixture of $\mathbf{3 r}$ and the linear regioisomer as a colorless oil $(32.3 \mathrm{mg}, 70 \%$, 3.3:1=branched:linear). The regioisomers were separated via $\mathrm{AgNO}_{3}$ impregnated silica preparative $\mathrm{TLC}$ 
(benzene) to afford $3 \mathrm{r}$ as a colorless oil for characterization; ${ }^{1} \mathrm{H}-\mathrm{NMR}\left(400 \mathrm{MHz}, \mathrm{CDCl}_{3}\right): \delta=7.34-7.27(\mathrm{~m}, 2 \mathrm{H})$, $7.24-7.16(\mathrm{~m}, 3 \mathrm{H}), 6.13(\mathrm{ddd}, J=17.1,10.1,7.5 \mathrm{~Hz}, 1 \mathrm{H}), 5.50-5.44(\mathrm{~m}, 1 \mathrm{H}), 5.13-5.06(\mathrm{~m}, 1 \mathrm{H}), 5.00(\mathrm{dt}, J=$ $17.1,1.5 \mathrm{~Hz}, 1 \mathrm{H}), 4.07(\mathrm{~d}, J=7.5 \mathrm{~Hz}, 1 \mathrm{H}), 2.40-2.31(\mathrm{~m}, 2 \mathrm{H}), 2.21-2.13(\mathrm{~m}, 2 \mathrm{H}), 1.93-1.78(\mathrm{~m}, 2 \mathrm{H}) ;{ }^{13} \mathrm{C}-\mathrm{NMR}$ $\left(100 \mathrm{MHz}, \mathrm{CDCl}_{3}\right): \delta=145.9,142.3,140.1,128.3,128.1,126.2,125.8,114.9,51.9,34.0,32.4,23.4 ;$ IR (neat): 3081, 3060, 3027, 2951, 2927, 2846, 1635, 1601, 1492, 1451, 1414, 1296, 1217, 1070, 1030, 994, 914, 799, 755, 719, 698, $667 \mathrm{~cm}^{-1}$; HRMS (EI): $\mathrm{m} / \mathrm{z}$ calcd for $\mathrm{C}_{14} \mathrm{H}_{16}$ [M] 184.1252 , found 184.1247; SFC: Daicel Chiracel OJ-H, $100 \% \mathrm{CO}_{2}, 2.0 \mathrm{~mL} / \mathrm{min}, 15^{\circ} \mathrm{C}, 200 \mathrm{~nm} ; 98 \%$ ee $\left(\mathrm{t}_{\mathrm{R}}(\right.$ major $)=10.0 \mathrm{~min}, \mathrm{t}_{\mathrm{R}}($ minor $\left.)=10.5 \mathrm{~min}\right) ;[\alpha]^{27}{ }_{\mathrm{D}}=+56.9(\mathrm{c}=$ $\left.1.0, \mathrm{CHCl}_{3}\right)$.

\section{(R)-(2-methylpenta-1,4-dien-3-yl)benzene (3s)}<smiles>C=CC(=C)C(=O)c1ccccc1</smiles>

The corresponding compound was prepared following the general procedure by using 1-phenylprop-2-en-1-ol 1a and potassium prop-1-en-2-yltrifluoroborate $2 \mathrm{~s}$. The product was isolated by silica plug filtration $\left(\mathrm{CH}_{2} \mathrm{Cl}_{2}\right)$, followed by preparative TLC (hex) to afford the product as a volatile colorless oil ( $24.8 \mathrm{mg}, 63 \%) ;{ }^{1} \mathrm{H}-\mathrm{NMR}$ (400 $\left.\mathrm{MHz}, \mathrm{CDCl}_{3}\right): \delta=7.35-7.28(\mathrm{~m}, 2 \mathrm{H}), 7.26-7.18(\mathrm{~m}, 3 \mathrm{H}), 6.05-5.95(\mathrm{~m}, 1 \mathrm{H}), 5.68-5.54(\mathrm{~m}, 2 \mathrm{H}), 5.14(\mathrm{dt}, J=$ $7.4,1.5 \mathrm{~Hz}, 1 \mathrm{H}), 5.10(\mathrm{~d}, J=1.5 \mathrm{~Hz}, 1 \mathrm{H}), 4.38(\mathrm{t}, J=7.4 \mathrm{~Hz}, 1 \mathrm{H}), 1.76-1.67(\mathrm{~m}, 3 \mathrm{H}) ;{ }^{13} \mathrm{C}-\mathrm{NMR}\left(100 \mathrm{MHz}, \mathrm{CDCl}_{3}\right): \delta$ $=143.6,140.4,131.6,128.5,127.6,126.2,124.6,114.5,46.6,13.0$; IR (neat): 3082, 3062, 3024, 2977, 2920 , 2857, 2362, 2344, 1635, 1600, 1492, 1451, 1398, 1370, 1074, 1030, 993, 913, 850, 757, 742, 719, 697, $669 \mathrm{~cm}^{-1}$; HRMS (EI): $\mathrm{m} / \mathrm{z}$ calcd for $\mathrm{C}_{12} \mathrm{H}_{14}[\mathrm{M}]^{+} 158.1096$, found 158.1090; SFC: Daicel Chiracel OJ-H, 1\% MeOH, 2.0 $\mathrm{mL} / \mathrm{min}, 25^{\circ} \mathrm{C}, 200 \mathrm{~nm} ; 94 \%$ ee $\left(\mathrm{t}_{\mathrm{R}}\right.$ (major) $\left.=9.8 \mathrm{~min}, \mathrm{t}_{\mathrm{R}}(\operatorname{minor})=12.3 \mathrm{~min}\right) ;[\alpha]^{24}{ }_{\mathrm{D}}=-62.1\left(\mathrm{c}=1.0, \mathrm{CHCl}_{3}\right)$.

\section{$(R, E)-(1-c y c l o h e x y l p e n t a-1,4-d i e n-3-y l)$ benzene (3t)}<smiles>C=CC(C=CC1CCCCC1)c1ccccc1</smiles>

The corresponding compound was prepared following the general procedure by using 1-phenylprop-2-en-1-ol 1a and potassium (E)-(2-cyclohexylvinyl)trifluoroborate $\mathbf{2 t}$. The product was isolated by flash chromatography (hexane) to afford the product as a colorless oil $(33.8 \mathrm{mg}, 60 \%) ;{ }^{1} \mathrm{H}-\mathrm{NMR}\left(400 \mathrm{MHz}, \mathrm{CDCl}_{3}\right): \delta=7.34-7.28(\mathrm{~m}$, $2 \mathrm{H}$ ), $7.24-7.18(\mathrm{~m}, 3 \mathrm{H}), 6.03$ (ddd, $J=17.0,10.2,6.8 \mathrm{~Hz}, 1 \mathrm{H}$ ), 5.59 (ddd, $J=15.5,6.8,1.1 \mathrm{~Hz}, 1 \mathrm{H}$ ), 5.47 (ddd, $J=$ $15.5,6.5,1.0 \mathrm{~Hz}, 1 \mathrm{H}), 5.14-5.09(\mathrm{~m}, 1 \mathrm{H}), 5.06(\mathrm{dt}, J=17.0,1.6 \mathrm{~Hz}, 1 \mathrm{H}), 4.00(\mathrm{t}, J=6.8 \mathrm{~Hz}, 1 \mathrm{H}), 2.05-1.93(\mathrm{~m}$, $1 \mathrm{H}), 1.79-1.69(\mathrm{~m}, 4 \mathrm{H}), 1.69-1.61(\mathrm{~m}, 1 \mathrm{H}), 1.35-1.03(\mathrm{~m}, 5 \mathrm{H}) ;{ }^{13} \mathrm{C}-\mathrm{NMR}\left(100 \mathrm{MHz}, \mathrm{CDCl}_{3}\right): \delta=143.5,141.0$, 137.6, 128.9, 128.3, 127.9, 126.2, 114.7, 52.1, 40.7, 33.1, 33.0, 26.2, 26.1; IR (neat): 3026, 2923, 2851, 1664 , 1636, 1600, 1493, 1448, 1217, 1073, 1030, 969, 915, 891, 753, 699, $667 \mathrm{~cm}^{-1}$; HRMS (EI): $\mathrm{m} / \mathrm{z}$ calcd for $\mathrm{C}_{17} \mathrm{H}_{22}$ $[\mathrm{M}]^{+}$226.1722, found 226.1716; SFC: Daicel Chirapak IB, $100 \% \mathrm{CO}_{2}, 2.0 \mathrm{~mL} / \mathrm{min}, 15^{\circ} \mathrm{C}, 200 \mathrm{~nm}$; $>99 \%$ ee $\left(\mathrm{t}_{R}\right.$ $($ major $)=4.7 \mathrm{~min}, \mathrm{t}_{\mathrm{R}}($ minor $\left.)=4.9 \mathrm{~min}\right) ;[\alpha]_{\mathrm{D}}^{27}=-3.3\left(\mathrm{c}=1.5, \mathrm{CHCl}_{3}\right)$. 


\section{$(R, E)$-(1-cyclopropylpenta-1,4-dien-3-yl)benzene (3u)}

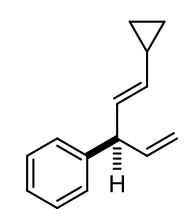

The corresponding compound was prepared following the general procedure by using 1-phenylprop-2-en-1-ol $\mathbf{1 a}$ and potassium $(E)$-(2-cyclopropylvinyl)trifluoroborate $\mathbf{2 u}$. The product was isolated by flash chromatography (hexane) to afford the product as a colorless oil $(23.5 \mathrm{mg}, 51 \%) ;{ }^{1} \mathrm{H}-\mathrm{NMR}\left(400 \mathrm{MHz}, \mathrm{CDCl}_{3}\right): \delta=7.35-7.28(\mathrm{~m}$, $2 \mathrm{H}), 7.27-7.17(\mathrm{~m}, 3 \mathrm{H}), 6.01(\mathrm{ddd}, J=17.1,10.2,6.9 \mathrm{~Hz}, 1 \mathrm{H}), 5.72(\mathrm{dd}, J=15.3,7.1 \mathrm{~Hz}, 1 \mathrm{H}), 5.14-4.99(\mathrm{~m}, 3 \mathrm{H})$, $\left.4.00(\mathrm{t}, J=6.9 \mathrm{~Hz}, 1 \mathrm{H}), 1.47-1.36(\mathrm{~m}, 1 \mathrm{H}), 0.74-0.64(\mathrm{~m}, 2 \mathrm{H}), 0.43-0.29(\mathrm{~m}, 2 \mathrm{H}) ;{ }^{13} \mathrm{C}-\mathrm{NMR}\left(100 \mathrm{MHz}^{\mathrm{C}} \mathrm{CDCl}\right)_{3}\right):$ $\delta=143.3,140.8,135.1,129.2,128.4,127.9,126.2,114.8,52.1,13.7,6.6$; IR (neat): 3081, 3061, 3025, 3004, $1661,1636,1600,1492,1451,1407,1097,1075,1046,1019,994,956,914,869,811,755,698,678 \mathrm{~cm}^{-1}$; HRMS (EI): $m / z$ calcd for $\mathrm{C}_{14} \mathrm{H}_{16}[\mathrm{M}]^{+} 184.1252$, found 184.1247; SFC: Daicel Chiracel OJ-H, $1 \% \mathrm{MeOH}, 2.0$ $\mathrm{mL} / \mathrm{min}, 25^{\circ} \mathrm{C}, 200 \mathrm{~nm}$; $99 \%$ ee $\left(t_{R}\right.$ (major) $=6.8 \mathrm{~min}, \mathrm{t}_{\mathrm{R}}$ (minor) $\left.=6.2 \mathrm{~min}\right) ;[\alpha]_{D}^{27}=-19.1\left(\mathrm{c}=1.0, \mathrm{CHCl}_{3}\right)$.

\section{Synthesis of (-)-Nyasol}<smiles>C=CC(O)c1ccc(OC)cc1</smiles>

$1 \mathrm{~m}$

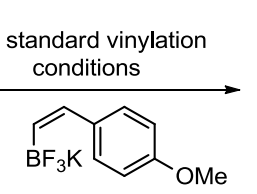

20<smiles>C=C[C@H](/C=C\c1ccc(OC)cc1)c1ccc(OC)cc1</smiles>

s1

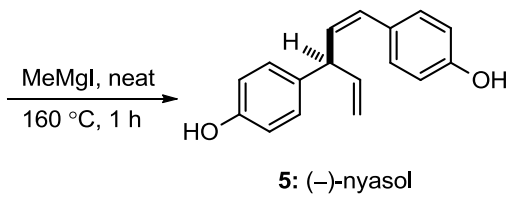

5: (-)-nyasol

$(R, Z)-4,4$ '-(penta-1,4-diene-1,3-diyl)bis(methoxybenzene) (S1):

$0.25 \mathrm{mmol}$ scale:

The corresponding compound was prepared following the general procedure by using 1-(4methoxyphenyl)prop-2-en-1-ol $1 \mathrm{~m}(0.25 \mathrm{mmol})$, potassium (Z)-(4-methoxystyryl)trifluoroborate 20 (1.1 equiv) and $\mathrm{HF}$ (1.0 equiv). The reaction mixture was purified via flash chromatography (hexane $/ \mathrm{CH}_{2} \mathrm{Cl}_{2}=2 / 1$ ) to afford the mixture of $\mathbf{S 1}$ and the linear regioisomer as a colorless oil (50.4 mg, 72\%, 3.8:1=branched:linear); The regioisomers were separated via $\mathrm{AgNO}_{3}$ impregnated silica preparative TLC (benzene) to afford S1 as a colorless oil; SFC: Daicel Chiracel OJ-H, 10\% MeOH, $2.0 \mathrm{~mL} / \mathrm{min}, 25^{\circ} \mathrm{C}, 200 \mathrm{~nm}$; $94 \%$ ee $\left(t_{R}\right.$ (major) = $17.9 \mathrm{~min}, \mathrm{t}_{\mathrm{R}}$ (minor) $=13.8 \mathrm{~min})$.

\section{$7.0 \mathrm{mmol}$ scale:}

$\left[\left\{\mid r(\operatorname{cod}) \mathrm{Cl}_{2}\right](0.141 \mathrm{~g}, 0.210 \mathrm{mmol}, 0.03\right.$ equiv) and $(S)-\mathrm{L}(0.426 \mathrm{~g}, 0.840 \mathrm{mmol}, 0.16$ equiv) were dissolved in technical grade 1,4-dioxane $(14.0 \mathrm{~mL})$ in a $50 \mathrm{~mL}$ polypropylene test tube and vigorously stirred for $15 \mathrm{~min}$. To the resulting dark red solution, 1-(4-methoxyphenyl)prop-2-en-1-ol 1m (1.15 g, $7.0 \mathrm{mmol}, 1.0$ equiv), potassium (Z)-(4-methoxystyryl)trifluoroborate 20 (1.85 g, $7.7 \mathrm{mmol}, 1.1$ equiv), $n \mathrm{Bu}_{4} \mathrm{NHSO}_{4}(0.238 \mathrm{~g}, 0.70 \mathrm{mmol}, 0.1$ equiv), and $\mathrm{HF}\left(0.27 \mathrm{~mL}, 7.7 \mathrm{mmol}, 1.1\right.$ equiv, $50 \%$ in $\left.\mathrm{H}_{2} \mathrm{O}\right)$ were added. The resulting heterogeneous orange mixture was stirred open to air at room temperature for $4 \mathrm{~h}$. The reaction mixture was concentrated under a reduced pressure and purified via flash chromatography (hexane $/ \mathrm{CH}_{2} \mathrm{Cl}_{2}=2: 1$ ) to afford the mixture of $\mathbf{S 1}$ and linear regioisomer as a colorless oil ( $1.29 \mathrm{~g}, 66 \%, 3.3: 1=$ branched:linear). A small amount of this mixture was separated via $\mathrm{AgNO}_{3}$ impregnated silica preparative TLC (benzene) to afford S1 as a colorless oil; ${ }^{1} \mathrm{H}-\mathrm{NMR}$ (400 $\left.\mathrm{MHz}, \mathrm{CDCl}_{3}\right): \delta=7.26-7.22(\mathrm{~m}, 2 \mathrm{H}), 7.20-7.14(\mathrm{~m}, 2 \mathrm{H}), 6.91-6.83(\mathrm{~m}, 4 \mathrm{H}), 6.55(\mathrm{~d}, J=11.4 \mathrm{~Hz}, 1 \mathrm{H}), 6.04(\mathrm{ddd}$, $J=17.2,10.2,6.1 \mathrm{~Hz}, 1 \mathrm{H}), 5.70(\mathrm{dd}, J=11.4,9.9 \mathrm{~Hz}, 1 \mathrm{H}), 5.19(\mathrm{dt}, J=10.2,1.6 \mathrm{~Hz}, 1 \mathrm{H}), 5.17-5.14(\mathrm{~m}, 1 \mathrm{H}), 4.53$ 
(dd, $J=9.9,6.1 \mathrm{~Hz}, 1 \mathrm{H}), 3.82(\mathrm{~s}, 3 \mathrm{H}), 3.80(\mathrm{~s}, 3 \mathrm{H}) ;{ }^{13} \mathrm{C}-\mathrm{NMR}\left(100 \mathrm{MHz}, \mathrm{CDCl}_{3}\right): \delta=158.5,158.1,140.8,135.5$, 131.7, 129.8, 129.7, 128.6, 128.5, 115.0, 114.0, 113.6, 55.3, 55.2, 46.9; IR (neat): 3003, 2953, 2933, 2906, 2834, 2357, 2059, 1888, 1607, 1574, 1508, 1463, 1441, 1301, 1242, 1173, 1109, 1032, 995, 967, 917, 875, 827, 760, $735,642,620 \mathrm{~cm}^{-1}$; HRMS (EI): $\mathrm{m} / \mathrm{z}$ calcd for $\mathrm{C}_{19} \mathrm{H}_{20} \mathrm{O}_{2}[\mathrm{MM}]^{+}$280.1463, found 280.1458; SFC: Daicel Chiracel OJ-H, $10 \% \mathrm{MeOH}, 2.0 \mathrm{~mL} / \mathrm{min}, 25^{\circ} \mathrm{C}, 200 \mathrm{~nm}$; 93\% ee $\left(t_{R}\right.$ (major) $=17.7 \mathrm{~min}, t_{R}($ minor $\left.)=13.7 \mathrm{~min}\right) ;[\alpha]^{24}{ }_{\mathrm{D}}=-204.5(\mathrm{c}$ $\left.=0.8, \mathrm{CHCl}_{3}\right)$.

\section{(-)-nyasol (5):}

To $(R, Z)-4,4$ '-(penta-1,4-diene-1,3-diyl)bis(methoxybenzene) (S1) $(41.9 \mathrm{mg}, 0.149 \mathrm{mmol}, 1.0$ equiv) in a $10 \mathrm{~mL}$ Schlenk tube was added $\mathrm{MeMgI}\left(149 \mu \mathrm{L}, 0.448 \mathrm{mmol}, 3.0\right.$ equiv, $3.0 \mathrm{M}$ in $\left.\mathrm{Et}_{2} \mathrm{O}\right)$ under an atmosphere of $\mathrm{N}_{2}$. $\mathrm{Et}_{2} \mathrm{O}$ was removed under a reduced pressure ( 400 torr), and the residue was heat at $160^{\circ} \mathrm{C}$ under a further reduced pressure ( $\sim 150$ torr) for $1 \mathrm{~h}$. After being cooled to the room temperature, the reaction was quenched by addition of a saturated aqueous $\mathrm{NH}_{4} \mathrm{Cl}$ solution $(3 \mathrm{~mL})$. The mixture was extracted with EtOAc $(3 \times 5 \mathrm{~mL})$, and the combined organic layers were washed with brine $(5 \mathrm{~mL})$, dried over $\mathrm{Na}_{2} \mathrm{SO}_{4}$, filtered and concentrated in vacuo. The crude product was purified by flash chromatography (pentane/acetone $=4 / 1$ ) to afford the product as a colorless viscous oil $(30 \mathrm{mg}, 80 \%) ;{ }^{1} \mathrm{H}-\mathrm{NMR}\left(400 \mathrm{MHz}, \mathrm{CDCl}_{3}\right): \delta=7.21-7.15(\mathrm{~m}, 2 \mathrm{H}), 7.13-7.08(\mathrm{~m}, 2 \mathrm{H})$, $6.82-6.75(\mathrm{~m}, 4 \mathrm{H}), 6.52(\mathrm{~d}, J=11.4 \mathrm{~Hz}, 1 \mathrm{H}), 6.07-5.96(\mathrm{~m}, 1 \mathrm{H}), 5.68(\mathrm{dd}, J=11.4,10.1 \mathrm{~Hz}, 1 \mathrm{H}), 5.18(\mathrm{dt}, J=7.1$, $1.6 \mathrm{~Hz}, 1 \mathrm{H}), 5.15(\mathrm{~d}, J=1.6 \mathrm{~Hz}, 1 \mathrm{H}), 4.97(\mathrm{~s}, 1 \mathrm{H}), 4.85(\mathrm{~s}, 1 \mathrm{H}), 4.50(\mathrm{dd}, J=10.1,6.1 \mathrm{~Hz}, 1 \mathrm{H}) ;{ }^{13} \mathrm{C}-\mathrm{NMR}(100 \mathrm{MHz}$, $\left.\mathrm{CDCl}_{3}\right): \delta=154.5,154.0,140.7,135.6,131.7,130.0,129.9,128.9,128.6,115.4,115.1,115.0,46.8 ;$ IR (neat): 3322 (br), 3012, 2974, 2926, 1695, 1610, 1510, 1443, 1368, 1232, 1171, 1103, 996, 919, 875, 832, 746, $645 \mathrm{~cm}^{-1}$; HRMS (ESI-): $\mathrm{m} / \mathrm{z}$ calcd for $\mathrm{C}_{17} \mathrm{H}_{15} \mathrm{O}_{2}[\mathrm{M}-\mathrm{H}]^{-}$251.1078, found 251.1077; SFC: Daicel Chirapak IA, 20\% MeOH, 2.0 $\mathrm{mL} / \mathrm{min}, 25^{\circ} \mathrm{C}, 200 \mathrm{~nm} ; 93 \%$ ee $\left(\mathrm{t}_{\mathrm{R}}(\right.$ major $)=7.6 \mathrm{~min}, \mathrm{t}_{\mathrm{R}}($ minor $\left.)=7.0 \mathrm{~min}\right) ;[\alpha]^{22}{ }_{\mathrm{D}}=-114.0\left(\mathrm{c}=1.0, \mathrm{CHCl}_{3}\right)$.

Characterization data of this compound were in accordance with previously reported values. ${ }^{10}$

\section{Synthesis of (-)-Hinokiresinol}

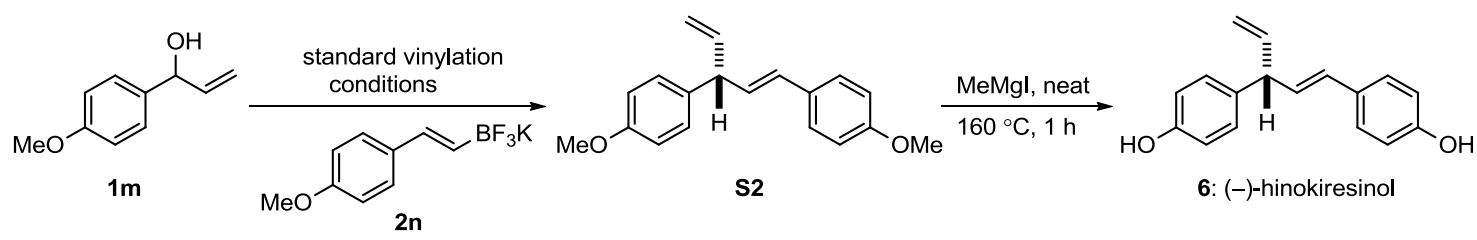

\section{$(R, E)-4,4$ '-(penta-1,4-diene-1,3-diyl)bis(methoxybenzene) (S2):}

The corresponding compound was prepared following the general procedure by using 1-(4methoxyphenyl)prop-2-en-1-ol $\mathbf{1 m}(0.4 \mathrm{mmol})$, potassium (E)-(4-methoxystyryl)trifluoroborate $\mathbf{2 n}$ (1.1 equiv) and $\mathrm{HF}$ (1.0 equiv). The reaction mixture was purified via flash chromatography (hexane $/ \mathrm{CH}_{2} \mathrm{Cl}_{2}=2 / 1$ ) to afford the mixture of $\mathbf{S 2}$ and the linear regioisomer as a colorless oil $(87.0 \mathrm{mg}, 78 \%, 18: 1=$ branched:linear); The regioisomers were separated via $\mathrm{AgNO}_{3}$ impregnated silica preparative TLC (benzene) to afford $\mathbf{S 2}$ as a colorless oil; ${ }^{1} \mathrm{H}-\mathrm{NMR}\left(400 \mathrm{MHz}, \mathrm{CDCl}_{3}\right): \delta=7.36-7.31(\mathrm{~m}, 2 \mathrm{H}), 7.24-7.19(\mathrm{~m}, 2 \mathrm{H}), 6.93-6.84(\mathrm{~m}, 4 \mathrm{H}), 6.40(\mathrm{~d}, \mathrm{~J}=16.0$ $\mathrm{Hz}, 1 \mathrm{H}$ ), $6.28(\mathrm{dd}, J=16.0,6.8 \mathrm{~Hz}, 1 \mathrm{H}$ ), 6.12 (ddd, $J=17.1,10.2,6.8 \mathrm{~Hz}, 1 \mathrm{H}), 5.19(\mathrm{dt}, J=10.2,1.6 \mathrm{~Hz}, 1 \mathrm{H}), 5.15$ $(\mathrm{dt}, J=17.1,1.6 \mathrm{~Hz}, 1 \mathrm{H}), 4.19(\mathrm{t}, J=6.8 \mathrm{~Hz}, 1 \mathrm{H}), 3.82(\mathrm{~s}, 3 \mathrm{H}), 3.82(\mathrm{~s}, 3 \mathrm{H}) ;{ }^{13} \mathrm{C}-\mathrm{NMR}\left(100 \mathrm{MHz}, \mathrm{CDCl}_{3}\right): \delta=158.9$, 158.2, 140.5, 134.8, 130.2, 129.9, 129.7, 129.0, 127.3, 115.1, 113.9, 55.2, 51.5; IR (neat): 3030, 3001, 2954, 2934, 2906, 2834, 2063, 1884, 1607, 1578, 1508, 1463, 1441, 1297, 1243, 1173, 1108, 1032, 968, 916, 826, 806, $778,749,664,638 \mathrm{~cm}^{-1}$; HRMS (EI): $\mathrm{m} / z$ calcd for $\mathrm{C}_{19} \mathrm{H}_{20} \mathrm{O}_{2}[\mathrm{M}]^{+}$280.1463, found 280.1458; SFC: Daicel Chiracel $\mathrm{OJ}-\mathrm{H}, 10 \% \mathrm{MeOH}, 2.0 \mathrm{~mL} / \mathrm{min}, 25^{\circ} \mathrm{C}, 200 \mathrm{~nm}$; $94 \%$ ee $\left(t_{R}\right.$ (major) $=21.0 \mathrm{~min}, \mathrm{t}_{\mathrm{R}}($ minor $\left.)=23.0 \mathrm{~min}\right) ;[\alpha]^{26}{ }_{\mathrm{D}}=-1.4$ $\left(\mathrm{c}=2.0, \mathrm{CHCl}_{3}\right)$. 


\section{(-)-hinokiresinol (6):}

To $(R, E)-4,4^{\prime}$-(penta-1,4-diene-1,3-diyl)bis(methoxybenzene) $\mathbf{S 2}(53.2 \mathrm{mg}, 0.190 \mathrm{mmol}, 1.0$ equiv) in a $10 \mathrm{~mL}$ -

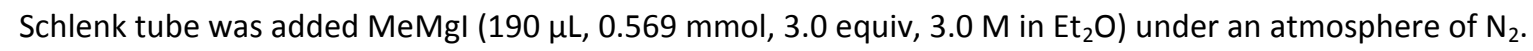
$\mathrm{Et}_{2} \mathrm{O}$ was removed under a reduced pressure ( $\sim 00 \mathrm{torr}$ ), and the residue was heat at $160^{\circ} \mathrm{C}$ under a further reduced pressure ( $\sim 150$ torr) for $1 \mathrm{~h}$. After being cooled to the room temperature, the reaction was quenched by addition of a saturated aqueous $\mathrm{NH}_{4} \mathrm{Cl}$ solution $(3 \mathrm{~mL})$. The mixture was extracted with EtOAc $(3 \times 5 \mathrm{~mL})$, and the combined organic layers were washed with brine $(5 \mathrm{~mL})$, dried over $\mathrm{Na}_{2} \mathrm{SO}_{4}$, filtered and concentrated in vacuo. The crude product was purified by flash chromatography (pentane/acetone $=4 / 1$ ) to afford the product as a white amorphous solid (41.2 mg, 86\%); ${ }^{1} \mathrm{H}-\mathrm{NMR}\left(400 \mathrm{MHz}, \mathrm{CDCl}_{3}\right): \delta=7.28-7.23(\mathrm{~m}, 2 \mathrm{H}), 7.15-7.10(\mathrm{~m}$, 2H), $6.82-6.74(\mathrm{~m}, 4 \mathrm{H}), 6.34(\mathrm{~d}, J=15.9 \mathrm{~Hz}, 1 \mathrm{H}), 6.22(\mathrm{dd}, J=15.9,6.8 \mathrm{~Hz}, 1 \mathrm{H}), 6.07$ (ddd, $J=17.0,10.2,6.8 \mathrm{~Hz}$, $1 \mathrm{H}), 5.16(\mathrm{dt}, J=10.2,1.5 \mathrm{~Hz}, 1 \mathrm{H}), 5.10(\mathrm{dt}, J=17.0,1.5 \mathrm{~Hz}, 1 \mathrm{H}), 4.81(\mathrm{br}, 2 \mathrm{H}), 4.14(\mathrm{t}, J=6.8 \mathrm{~Hz}, 1 \mathrm{H}) ;{ }^{13} \mathrm{C}-\mathrm{NMR}$ (100 MHz, $\left.\mathrm{CDCl}_{3}\right): \delta=154.7,154.0,140.4,135.0,130.4,129.9,129.7,129.2,127.5,115.4,115.3,115.2,51.4 ; \mathrm{IR}$ (neat): 3304 (br), 3021, 2926, 2856, 2702, 1883, 1633, 1609, 1604, 1509, 1441, 1364, 1216, 1170, 1102, 968, 918, 830, 799, 752, $667 \mathrm{~cm}^{-1}$; HRMS (ESI-): $\mathrm{m} / \mathrm{z}$ calcd for $\mathrm{C}_{17} \mathrm{H}_{15} \mathrm{O}_{2}[\mathrm{M}-\mathrm{H}]^{-}$251.1078, found 251.1075; SFC: Daicel Chirapak IA, 15\% MeOH, $2.0 \mathrm{~mL} / \mathrm{min}, 25^{\circ} \mathrm{C}, 200 \mathrm{~nm}$; 94\% ee $\left(\mathrm{t}_{\mathrm{R}}\right.$ (major) $=16.2 \mathrm{~min}, \mathrm{t}_{\mathrm{R}}$ (minor) $\left.=17.4 \mathrm{~min}\right)$; $[\alpha]_{D}^{23}=-5.3\left(\mathrm{c}=1.0, \mathrm{CHCl}_{3}\right)$.

Characterization data of this compound were in accordance with previously reported values. ${ }^{11}$

\section{References}

(1) Li, T.-S.; Li, J.-T.; Li, H.-Z. J. Chromatogr., A 1995, 715, 372.

(2) Defieber, C.; Ariger, M. A.; Moriel, P.; Carreira, E. M. Angew. Chem., Int. Ed. 2007, 46, 3139.

(3) Lafrance, M.; Roggen, M.; Carreira, E. M. Angew. Chem., Int. Ed. 2012, 51, 3470.

(4) Roggen, M.; Carreira, E. M. Angew. Chem. Int., Ed. 2011, 50, 5568.

(5) Roggen, M.; Carreira E. M. Angew. Chem., Int. Ed. 2012, 51, 8652.

(6) Molander, G. A.; Rodriguez-Rivero, M. Org. Lett. 2002, 4, 107.

(7) Molander, G. A.; Bernardi, C. R. J. Org. Chem. 2002, 67, 8424.

(8) Molander, G. A.; Felix, L. A. J. Org. Chem. 2005, 70, 3950.

(9) Molander, G. A.; Ellis, N. M. J. Org. Chem. 2008, 73, 6841.

(10) Akiyama, K.; Gao, F.; Hoveyda, A. H. Angew. Chem., Int. Ed. 2010, 49, 419.

(11) Lassen, P. R.; Skytte, D. M.; Hemmngsen, L.; Nielsen, S. F.; Freedman, T. B.; Nafie, L. A.; Christensen, S. B. J. Nat. Prod. 2005, 68, 1603. 
SFC Data
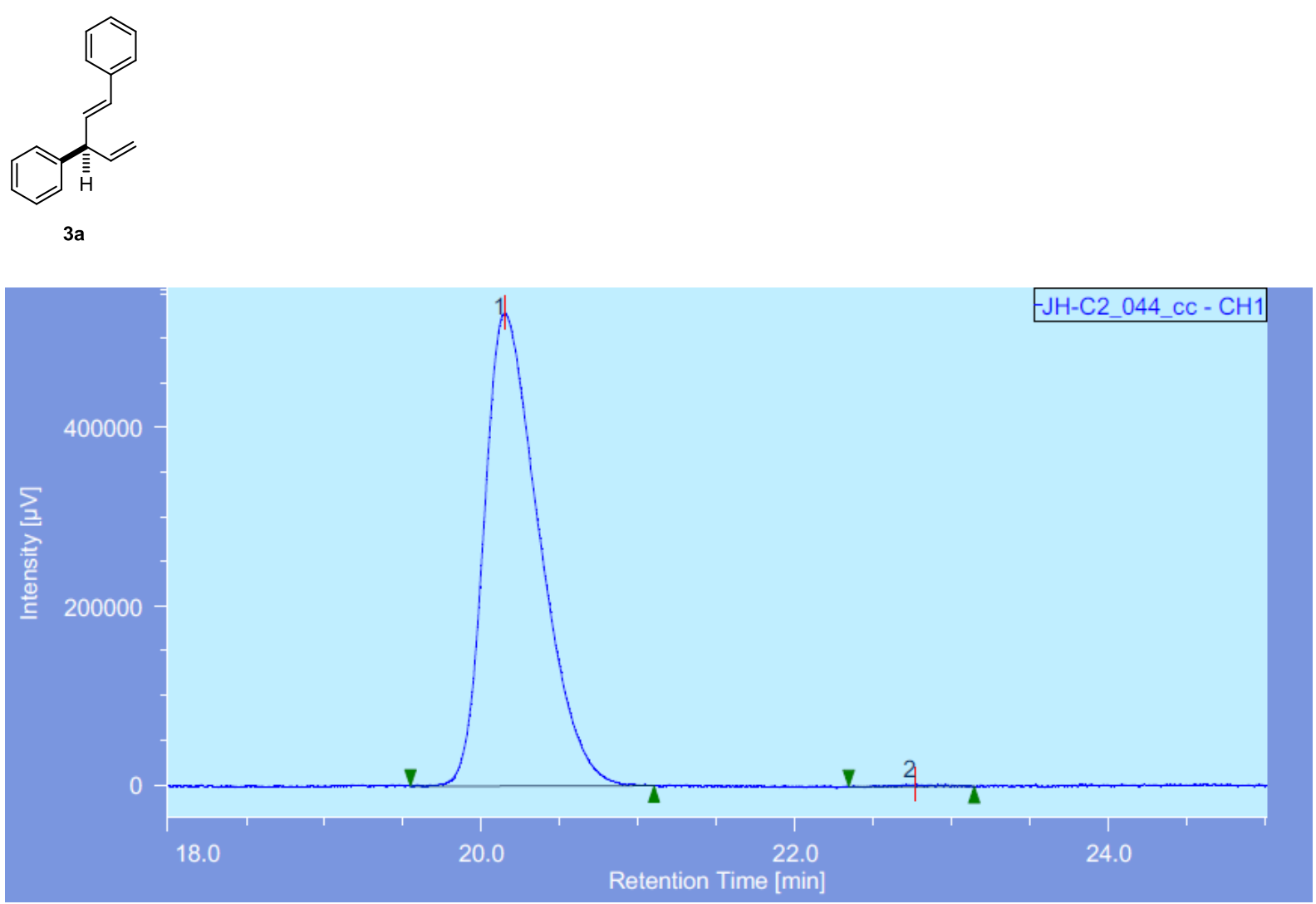

\begin{tabular}{|c|c|c|c|c|c|c|c|c|c|c|c|}
\hline \begin{tabular}{l|l}
$\#$ & Peak Name \\
\end{tabular} & $\mathrm{CH}$ & $\mathrm{tR}$ [min] & Area $[\mu \mathrm{V} \cdot \mathrm{sec}]$ & Height $[\mu \mathrm{V}]$ & Area $\%$ & Height $\%$ & Quantity & NTP & Resolution & Symmetry Factor & Warning \\
\hline 1 Unknown & 1 & 20.150 & 12911216 & 529148 & 99.732 & 99.547 & $\mathrm{~N} / \mathrm{A}$ & 15756 & 4.035 & 1.455 & \\
\hline 2 Unknown & 1 & 22.767 & 34693 & 2406 & 0.268 & 0.453 & $\mathrm{~N} / \mathrm{A}$ & 19136 & $\mathrm{~N} / \mathrm{A}$ & 0.944 & \\
\hline
\end{tabular}

\section{Racemic}

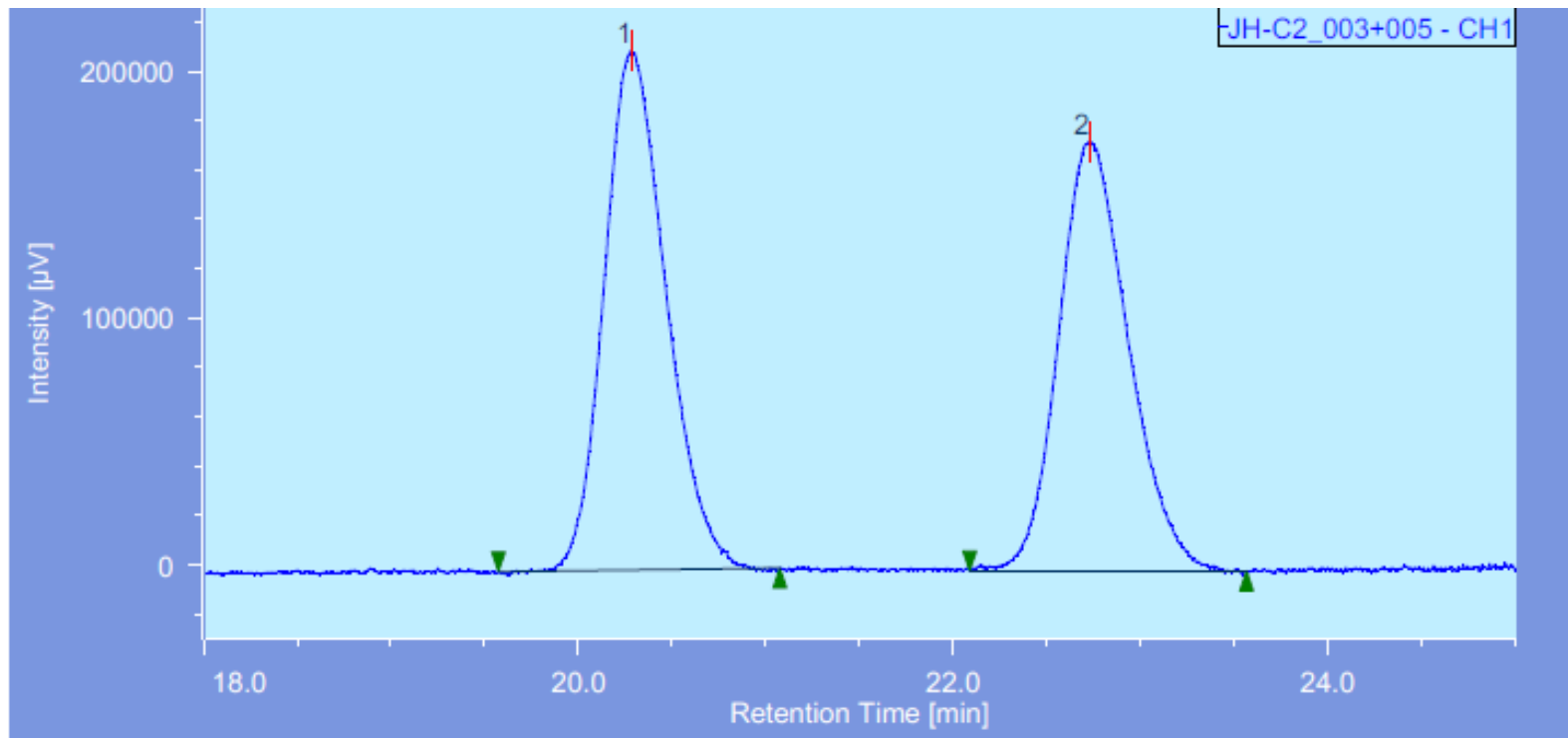

\begin{tabular}{|r|c|c|r|r|r|r|r|r|r|r|r|r|}
\hline$\#$ & Peak Name & $\mathrm{CH}$ & $\mathrm{tR}[\mathrm{min}]$ & Area $[\mu \mathrm{V} \cdot \mathrm{sec}]$ & Height $[\mu \mathrm{V}]$ & \multicolumn{1}{|c|}{ Area $\%$} & Height $\%$ & Quantity & NTP & Resolution & Symmetry Factor & Warning \\
\hline 1 & Unknown & 1 & 20.283 & 4840621 & 210523 & 51.72 & 54.755 & N/A & 18174 & 3.827 & 1.216 & \\
\hline 2 & 1 & 22.725 & 4518593 & 173958 & 48.28 & 45.245 & N/A & 17999 & N/A & & 1.208 & \\
\hline
\end{tabular}




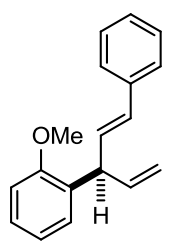

3b

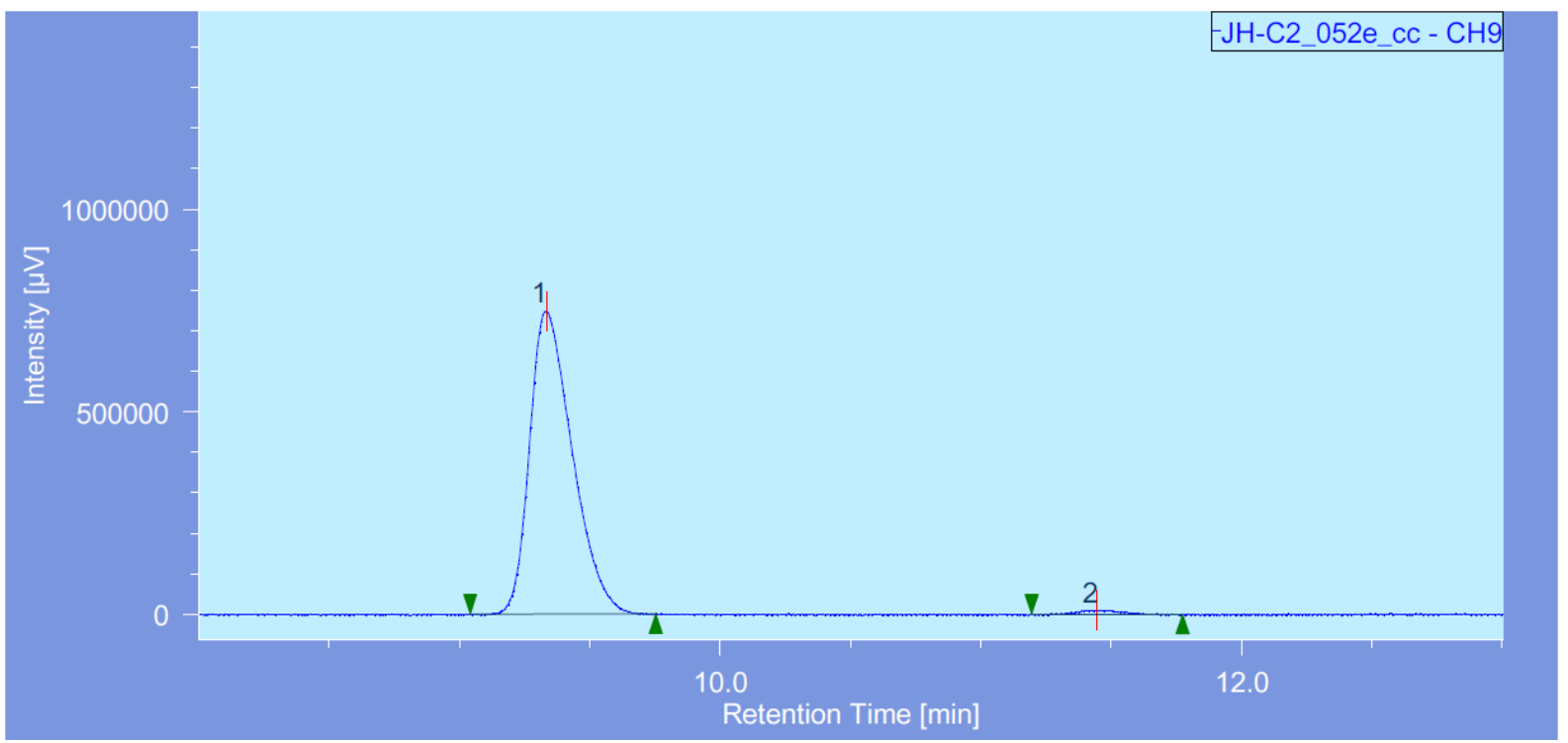

\begin{tabular}{|c|c|c|c|c|c|c|c|c|c|c|c|}
\hline \begin{tabular}{l|l}
$\#$ & Peak Name \\
\end{tabular} & $\mathrm{CH}$ & $\mathrm{tR}[\min ]$ & Area $[\mu \mathrm{V} \cdot \mathrm{sec}]$ & Height $[\mu \mathrm{V}]$ & Area\% & Height \% & Quantity & NTP & Resolution & Symmetry Factor & Warning \\
\hline Unknown & 9 & 9.333 & 8470389 & 748218 & 98.736 & 98.71 & $\mathrm{~N} / \mathrm{A}$ & $1576:$ & 6.722 & 1.393 & \\
\hline Unknown & 9 & 11.447 & 108396 & 9757 & 1.264 & 1.28 & $\mathrm{~N} / \mathrm{A}$ & 18893 & N/A & 1.296 & \\
\hline
\end{tabular}

\section{Racemic}

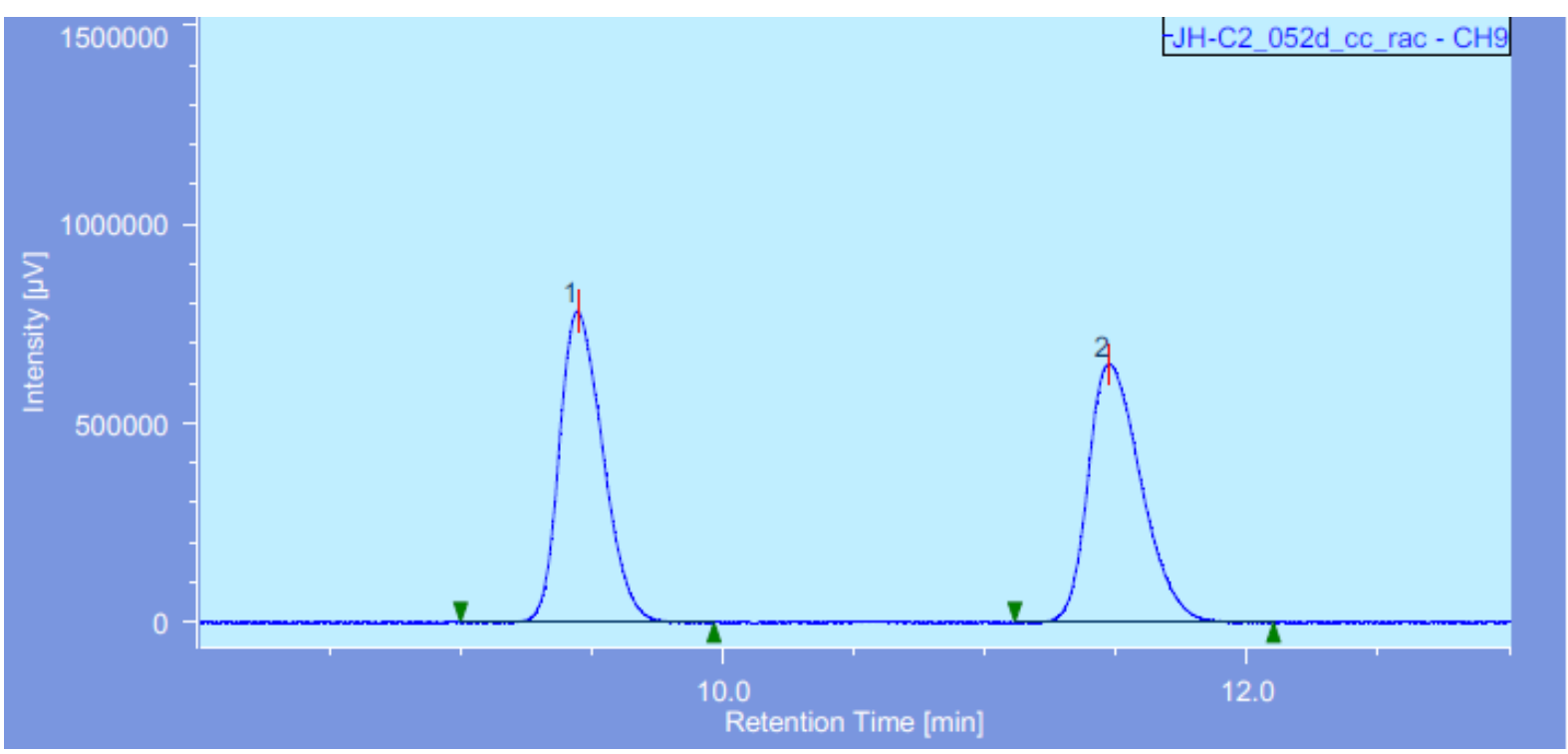

\begin{tabular}{|c|c|c|c|c|c|c|c|c|c|c|c|c|}
\hline$\#$ & Peak Name & $\mathrm{CH}$ & $t R[\min ]$ & Area [ $\mu \mathrm{V}-\sec ]$ & Height $[\mu \mathrm{V}]$ & Area $\%$ & Height $\%$ & Quantity & NTP & Resolution & Symmetry Factor & Warning \\
\hline & Unknown & 9 & 9.447 & 8958657 & 782333 & 49.495 & 54.643 & $\mathrm{~N} / \mathrm{A}$ & 15393 & 6.031 & 1.270 & \\
\hline & Unknown & 9 & 11.473 & 9141286 & 649392 & 50.505 & 45.357 & N/A & 15441 & N/A & 1.344 & \\
\hline
\end{tabular}



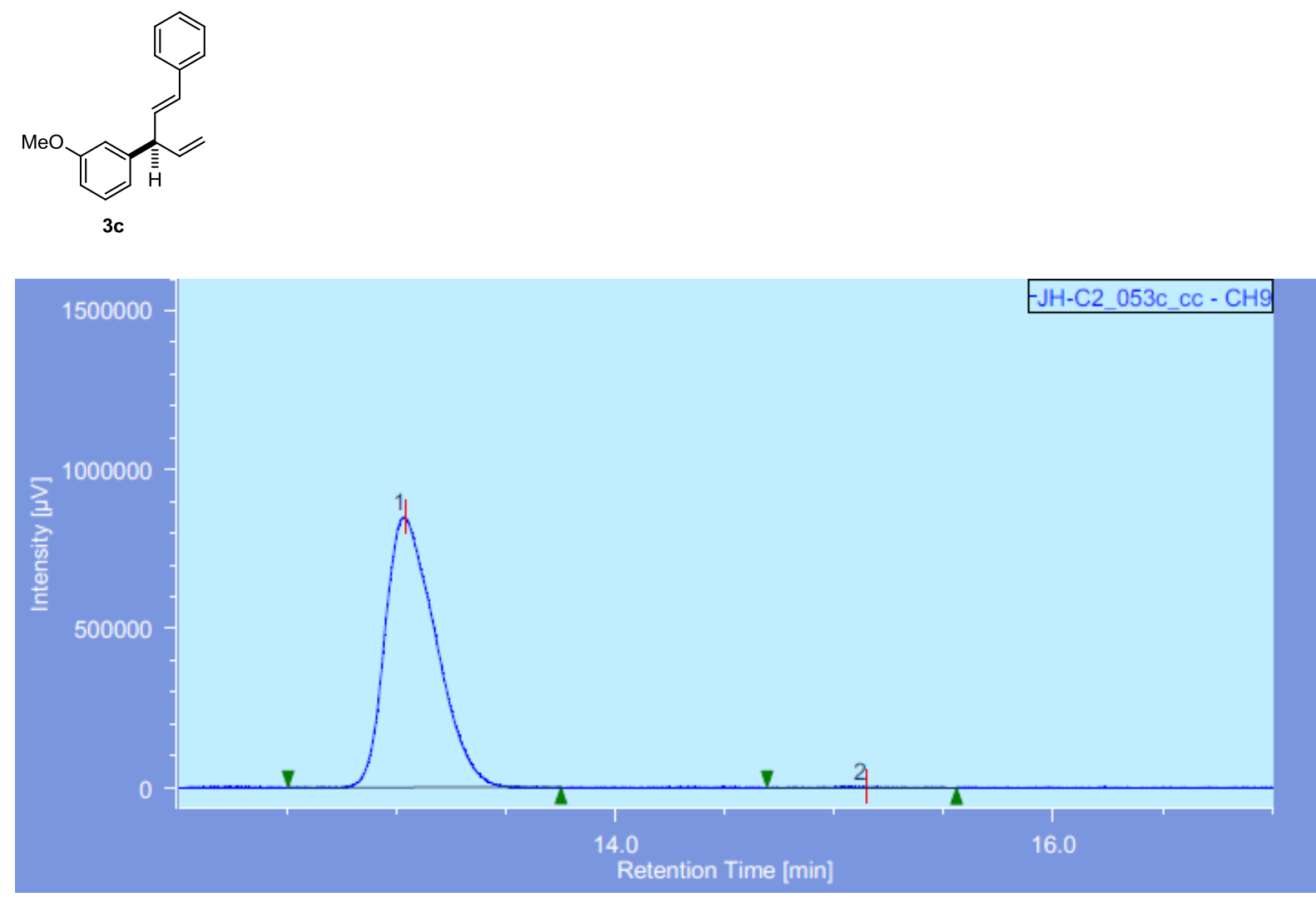

\begin{tabular}{|c|c|c|c|c|c|c|c|c|c|c|c|}
\hline \begin{tabular}{|l|l|}
$\#$ & Peak Name \\
\end{tabular} & $\mathrm{CH}$ & tR [min] & Area $\left[\mu \mathrm{V}_{\mathrm{sec}}\right]$ & Height $[\mu \mathrm{V}]$ & Area $\%$ & Height $\%$ & Quantity & NTP & Resolution & Symmetry Factor & Warning \\
\hline 1 Unknown & 9 & 13.040 & 13607098 & 848495 & 99.81 & 99.588 & N/A & 14579 & 6.245 & 1.391 & \\
\hline 2 Unknown & 9 & 15.14 & 25739 & 3508 & 0.18 & 0.412 & $\mathrm{~N} / \mathrm{A}$ & $6140 \Omega$ & $\mathrm{N} / \mathrm{A}$ & 0.956 & \\
\hline
\end{tabular}

\section{Racemic}

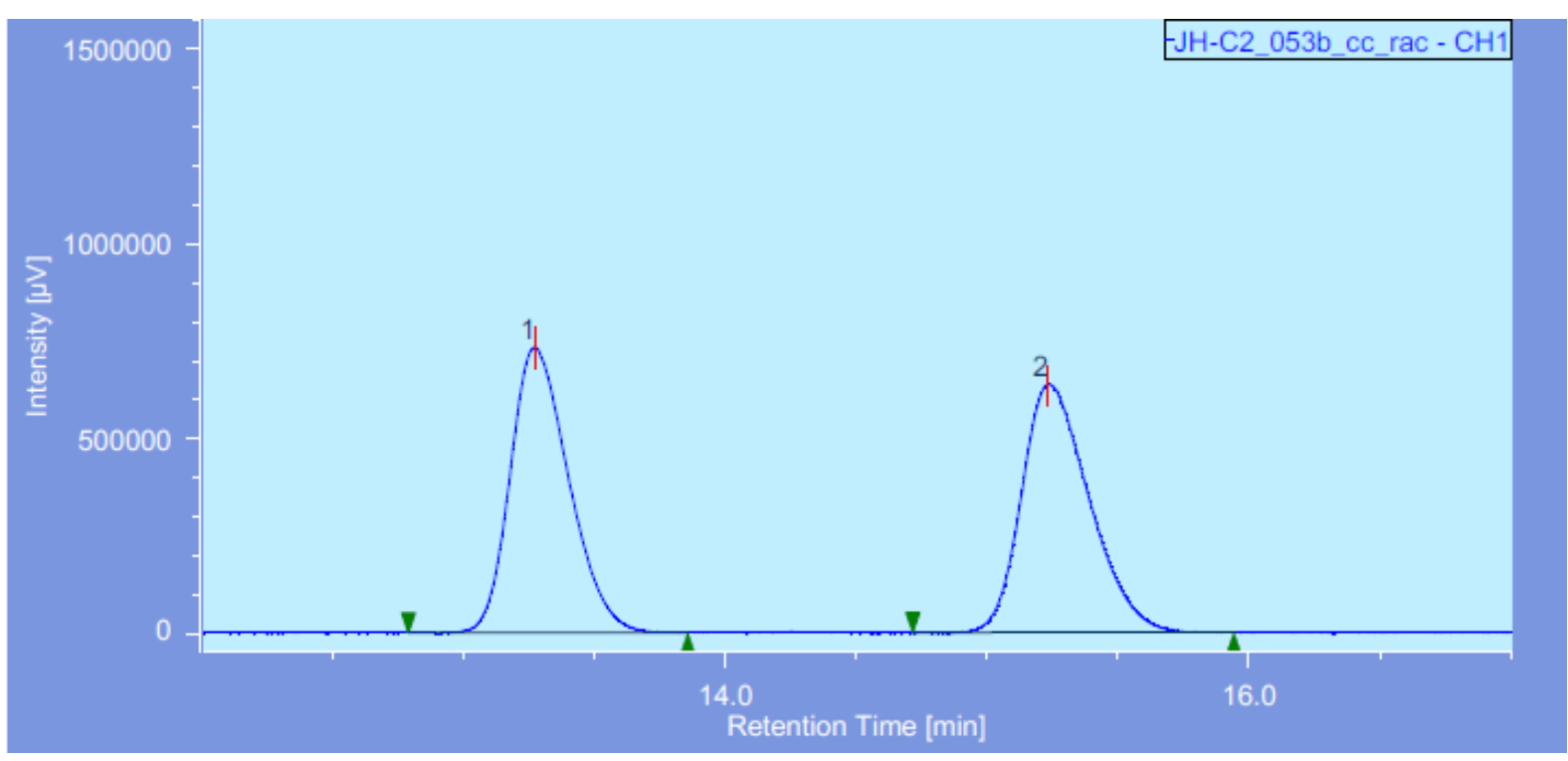

\begin{tabular}{|c|c|c|c|c|c|c|c|c|c|c|c|}
\hline Peak Name & $\mathrm{CH}$ & $t \mathbb{R}[\min ]$ & Area $[\mu \mathrm{V}$-sec] & 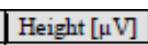 & Area\% & Height\% & Quantity & NTP & \begin{tabular}{|l|} 
Resolution \\
\end{tabular} & Symmetry Factor & Waming \\
\hline Nknown & 1 & 13.27 & 11311233 & 7297 & 49.876 & 53.538 & N/A & 16972 & 4.464 & 1.258 & \\
\hline Unknown & 1 & 15.233 & 11367581 & 63329 & 50.124 & 46.462 & & 16656 & N/A & 1.32 & \\
\hline
\end{tabular}



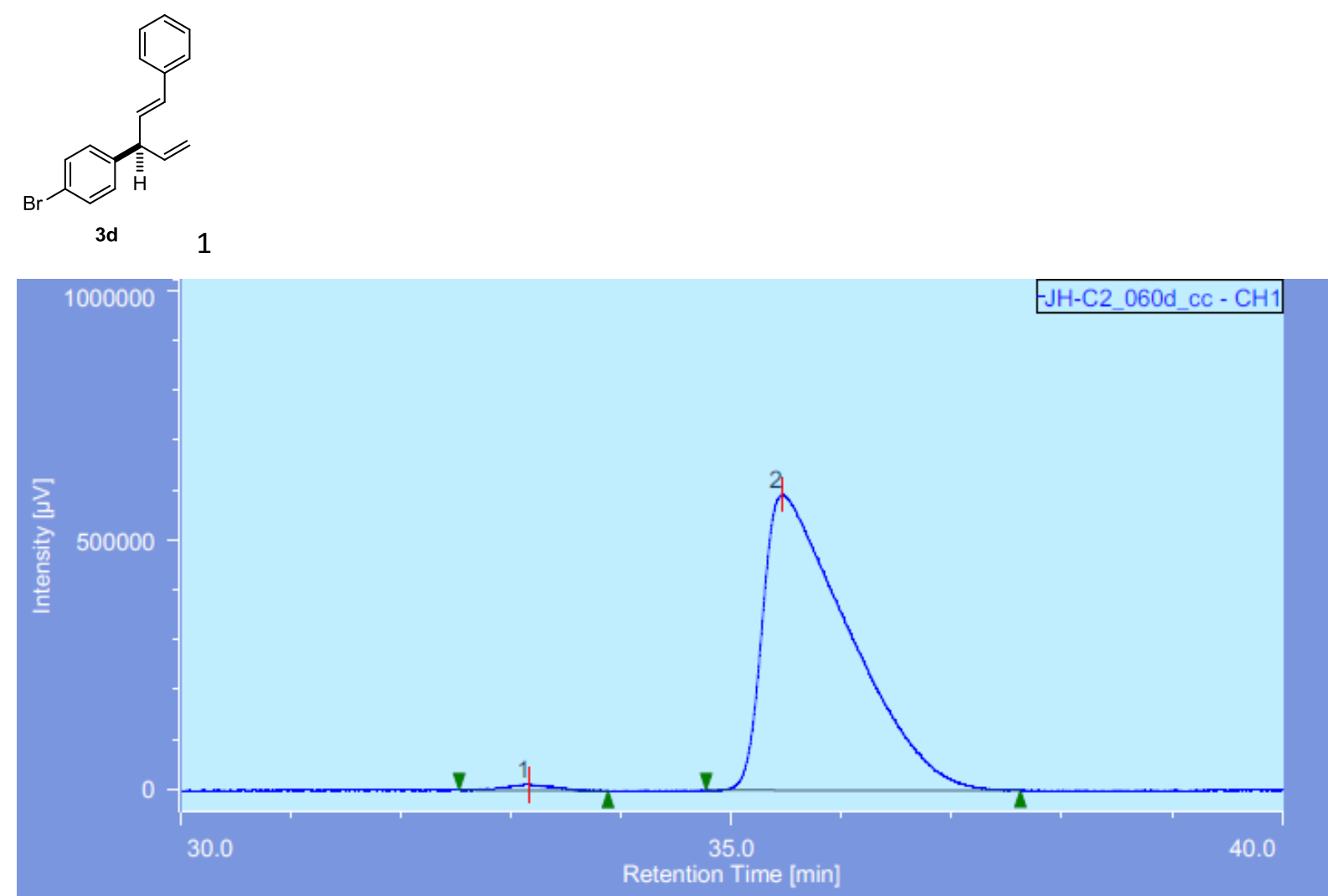

\begin{tabular}{|c|c|c|c|c|c|c|c|c|c|c|c|}
\hline Pealk Name & $\mathrm{CH}$ & $\mathbb{t R}[\min ]$ & Area $[\mu \mathrm{V}$-sec $]$ & Height $[\mu \mathrm{V}]$ & Area\% & Height\% & Quantity & NIP & \begin{tabular}{|l|} 
Resolution \\
\end{tabular} & Symmetry Factor & Waming \\
\hline Jnknown & 1 & 33.16 & 365133 & 1100 & 1.12 & 1.825 & N/A & 22386 & 1.969 & 1.076 & \\
\hline Unknown & & 35.458 & 2204450 & 592140 & 98.879 & 98.175 & N/A & 9610 & N/A & & \\
\hline
\end{tabular}

\section{Racemic}

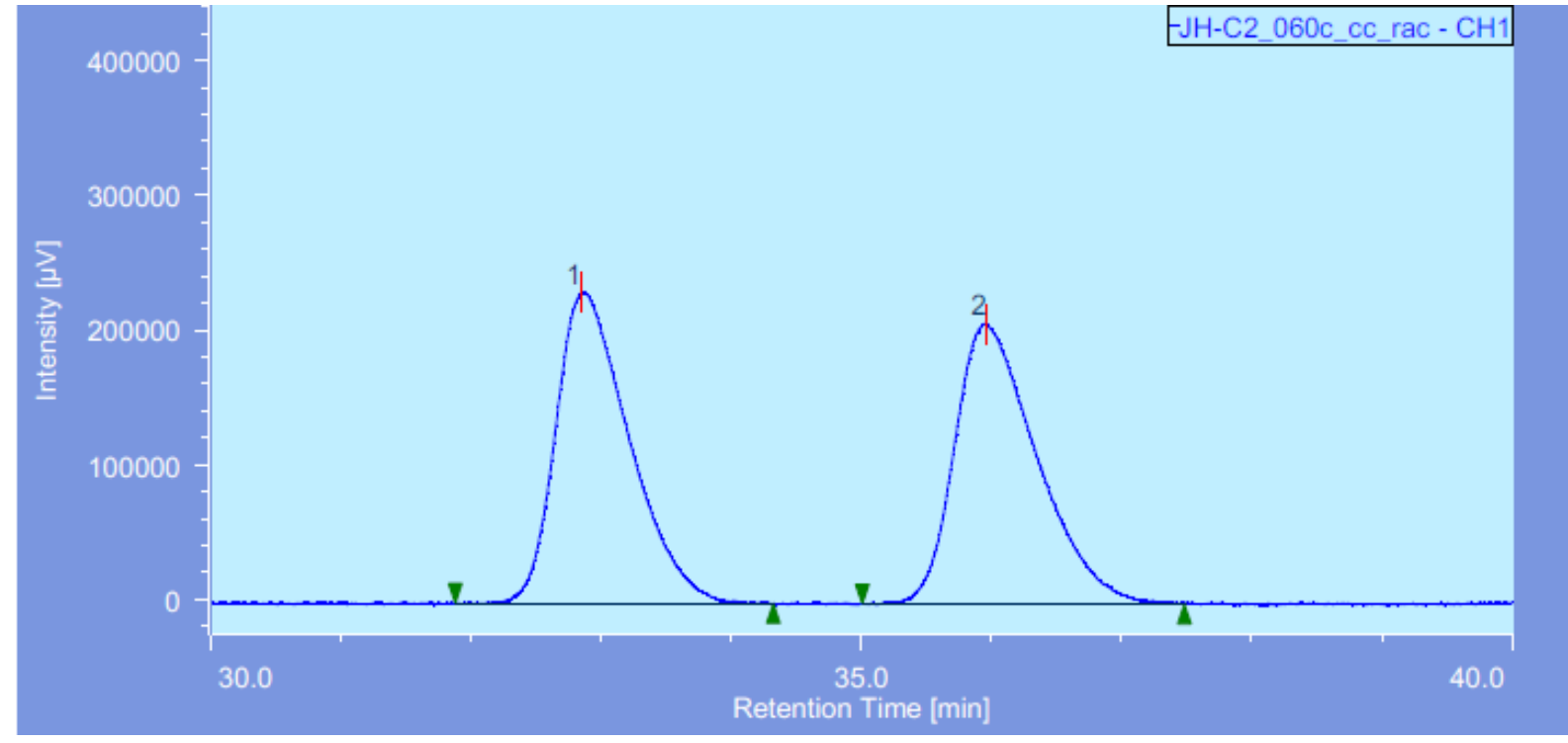

\begin{tabular}{|c|c|c|c|c|c|c|c|c|c|c|c|c|}
\hline$\pi$ & Peak Name & $\mathrm{CH}$ & $t R[\min ]$ & Area $[\mu \mathrm{V}-\sec ]$ & Height $[\mu \mathrm{V}]$ & Area\% & Height $\%$ & Quantity & NTP & Resolution & Symmetry Factor & Warning \\
\hline & Unknown & 1 & 32.850 & 9014944 & 230276 & 49.746 & 52.563 & $\mathrm{~N} / \mathrm{A}$ & 16524 & 2.881 & 1.483 & \\
\hline & Unknown & 1 & 35.958 & 9106965 & 207815 & 50.254 & 47.437 & $\mathrm{~N} / \mathrm{A}$ & 15876 & $\mathrm{~N} / \mathrm{A}$ & 1.440 & \\
\hline
\end{tabular}



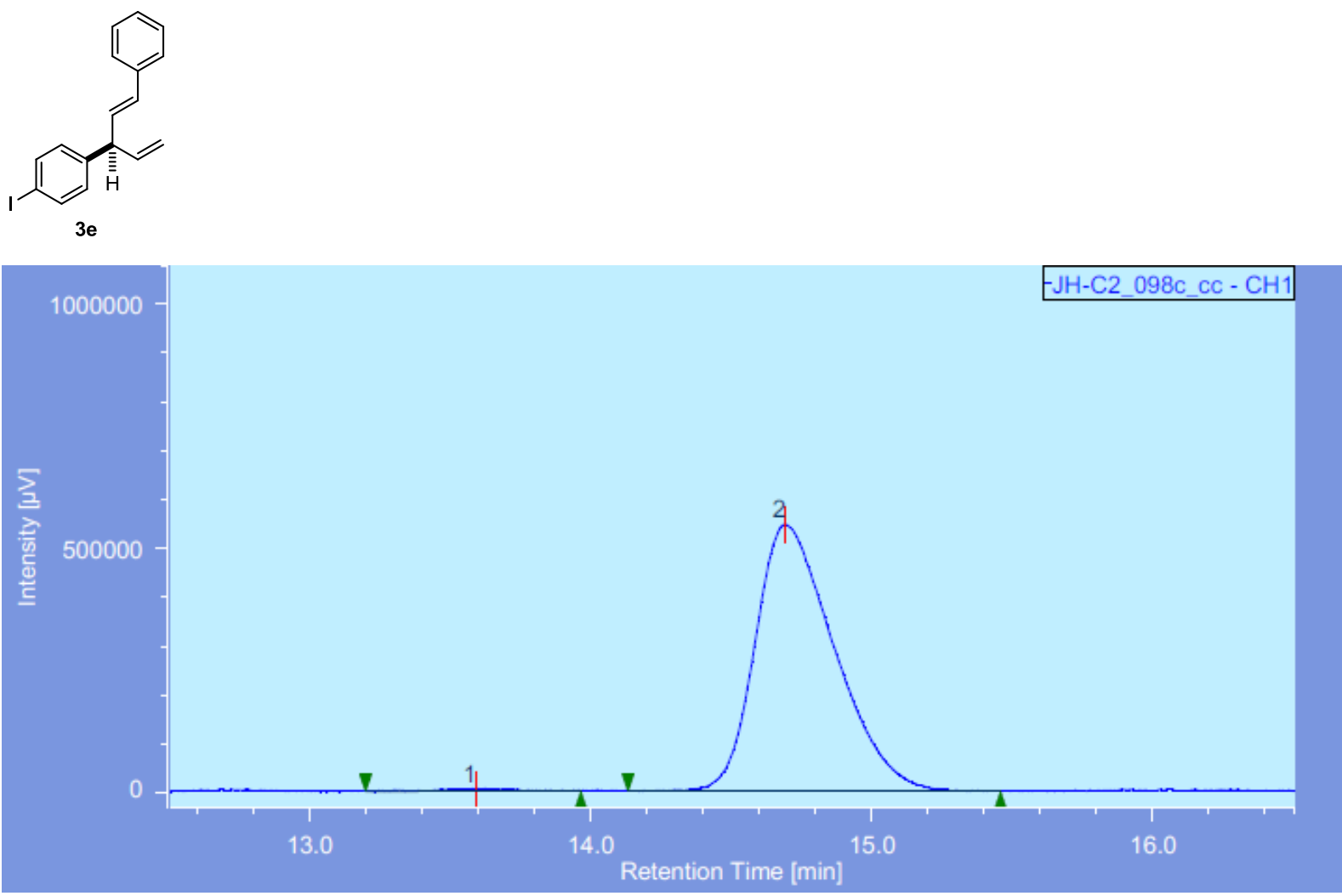

\begin{tabular}{|c|c|c|c|c|c|c|c|c|c|c|c|}
\hline \begin{tabular}{l|l}
$\#$ Peak Name \\
\end{tabular} & $\mathrm{CH}$ & $t \mathbb{R}[\min ]$ & Area $[\mu \mathrm{V}-\mathrm{sec}]$ & Height $[\mu \mathrm{V}]$ & Area\% & Height\% & Quantity & NTP & Resolution & Symmetry Factor & Warn \\
\hline Unknown & 1 & 13.592 & 64987 & 4338 & 0.609 & 0.792 & N/A & 14930 & 2.300 & 0.862 & \\
\hline Unknown & 1 & 14.692 & 10601159 & 543358 & 99.391 & 99.208 & $\mathrm{~N} / \mathrm{A}$ & 13059 & $\mathrm{~N} / \mathrm{A}$ & 1.401 & \\
\hline
\end{tabular}

\section{Racemic}

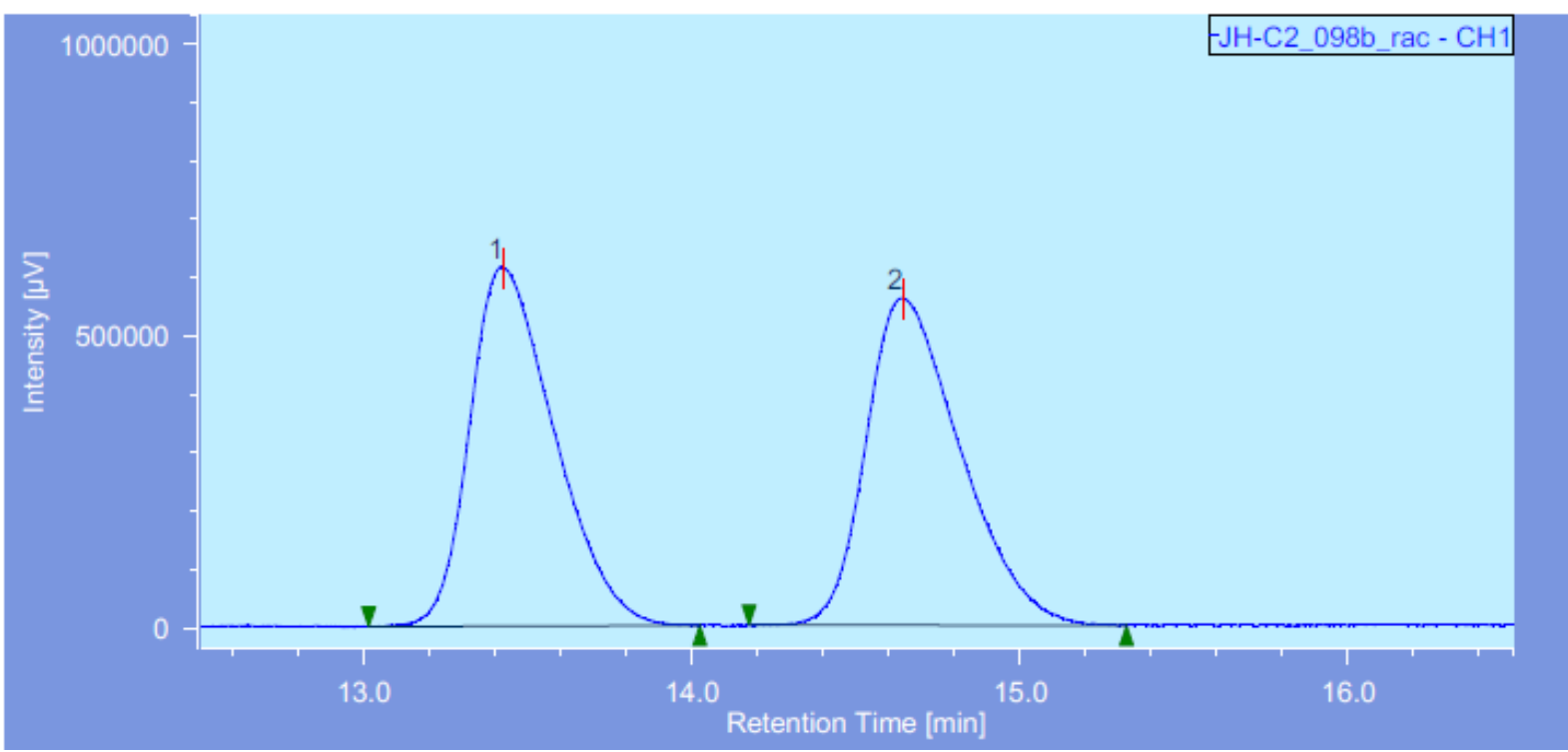

\begin{tabular}{|c|c|c|c|c|c|c|c|c|c|c|c|c|}
\hline$\#$ & Peak Name & $\mathrm{CH}$ & $t \mathbb{R}[\min ]$ & Area $[\mu \mathrm{V}-\mathrm{sec}]$ & Height $[\mu \mathrm{V}]$ & Area\% & Height $\%$ & Quantity & NIP & Resolution & Symmetry Factor & Warning \\
\hline & Unknown & 1 & 13.425 & 10999220 & 613610 & 49.541 & 52.289 & $\mathrm{~N} / \mathrm{A}$ & 12873 & 2.441 & 1.371 & \\
\hline & Unknown & 1 & 14.642 & 11202919 & 559895 & 50.459 & 47.711 & $\mathrm{~N} / \mathrm{A}$ & 12393 & $\mathrm{~N} / \mathrm{A}$ & 1.386 & \\
\hline
\end{tabular}



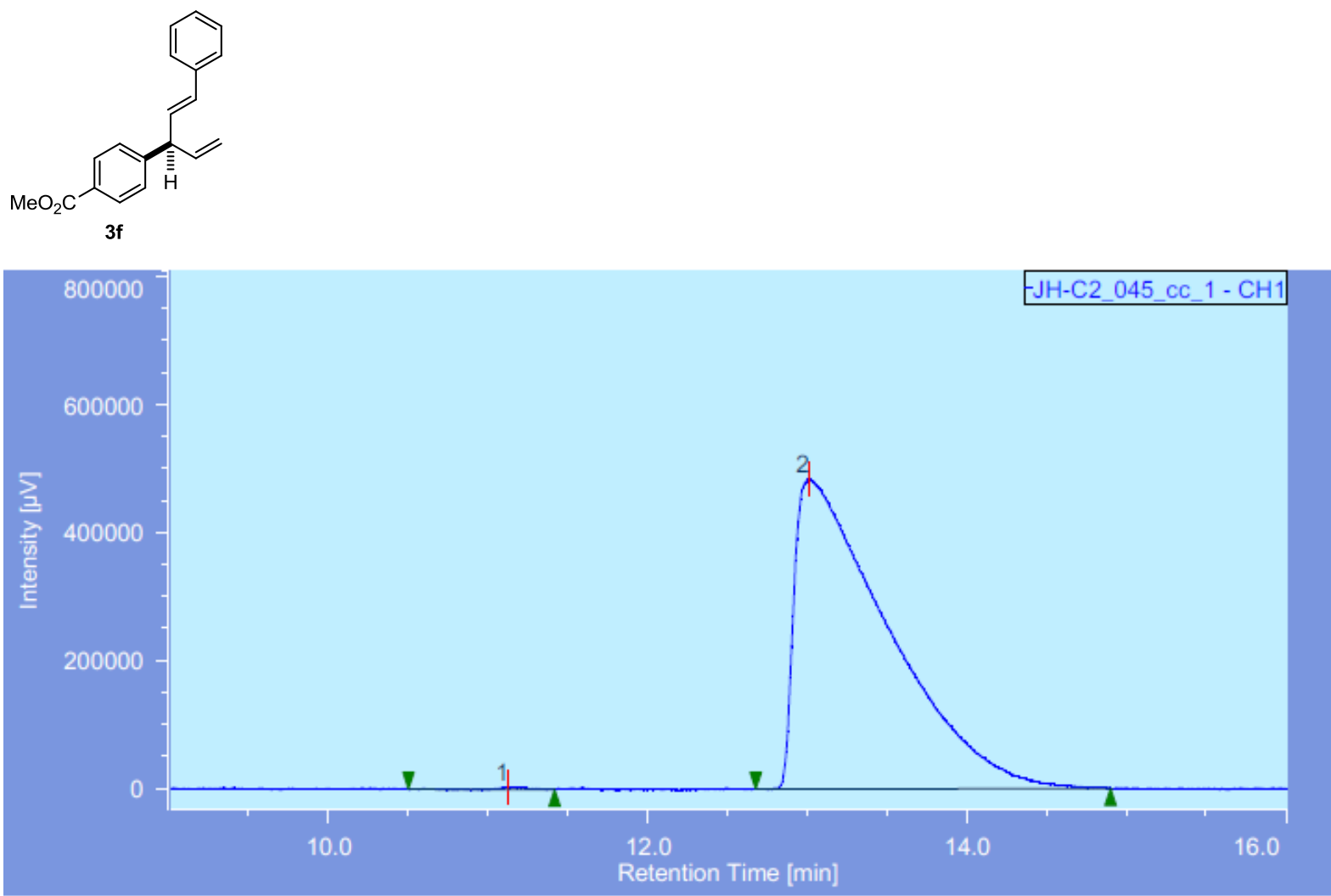

\begin{tabular}{|c|c|c|c|c|c|c|c|c|c|c|c|}
\hline \begin{tabular}{l|l}
$\#$ & Peak Name \\
\end{tabular} & $\mathrm{CH}$ & $t R[\min ]$ & Area $[\mu \mathrm{V}$-sec $]$ & Height $[\mu \mathrm{V}]$ & Area $\%$ & Height\% & Quantity & NTP & Resolution & Symmetry Factor & Wami \\
\hline Unknown & 1 & 11.12 & 48355 & 3565 & 0.24 & 0.73 & N/A & 1912 & 2.733 & 0.73 & \\
\hline Unknown & \begin{tabular}{|l|l|}
1 \\
\end{tabular} & 13.005 & 19712184 & 483702 & 99.75 & 99.26 & N/A & 241 & N/A & 4.50. & \\
\hline
\end{tabular}

\section{Racemic}

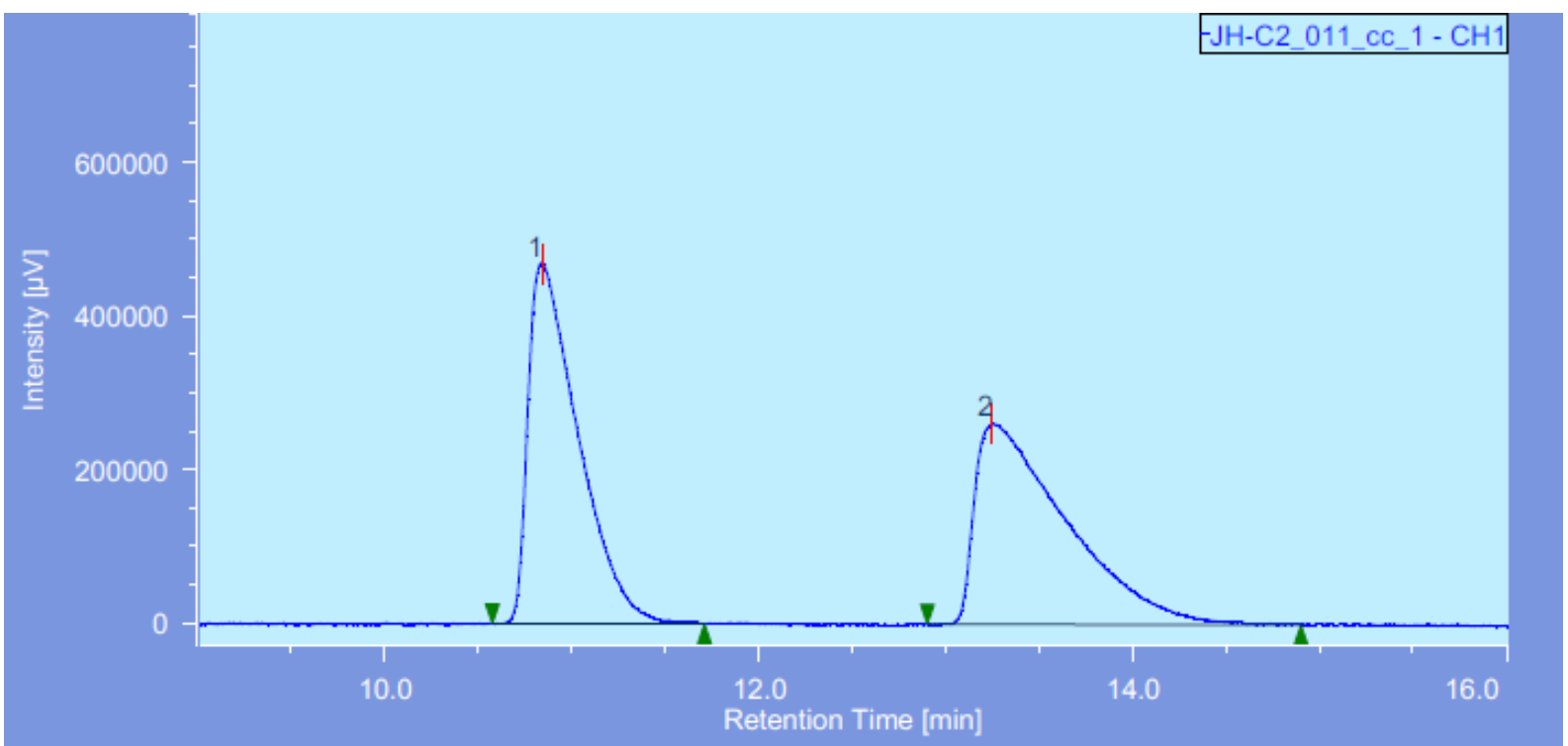

\begin{tabular}{|c|c|c|c|c|c|c|c|c|c|c|c|}
\hline Peak Nam & $\mathrm{CH}$ & $t R[\min ]$ & Area $[\mu \mathrm{V}-\mathrm{sec}]$ & Height $[\mu \mathrm{V}]$ & Area\% & Height\% & Quantity & NTP & lution & Symmetry Factor & \begin{tabular}{|l|} 
Wanning \\
\end{tabular} \\
\hline Unknown & 1 & 10.842 & 8707858 & 467901 & 49.607 & 64.216 & $\mathrm{~N} / \mathrm{A}$ & $794 \pi$ & $3.53 \mathrm{~T}$ & 2.211 & \\
\hline Naniown & & 13.250 & $=$ & & 50.393 & 35.784 & -4 & 3635 & -1 & 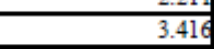 & \\
\hline
\end{tabular}




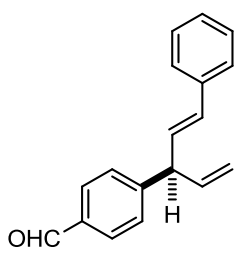

3g

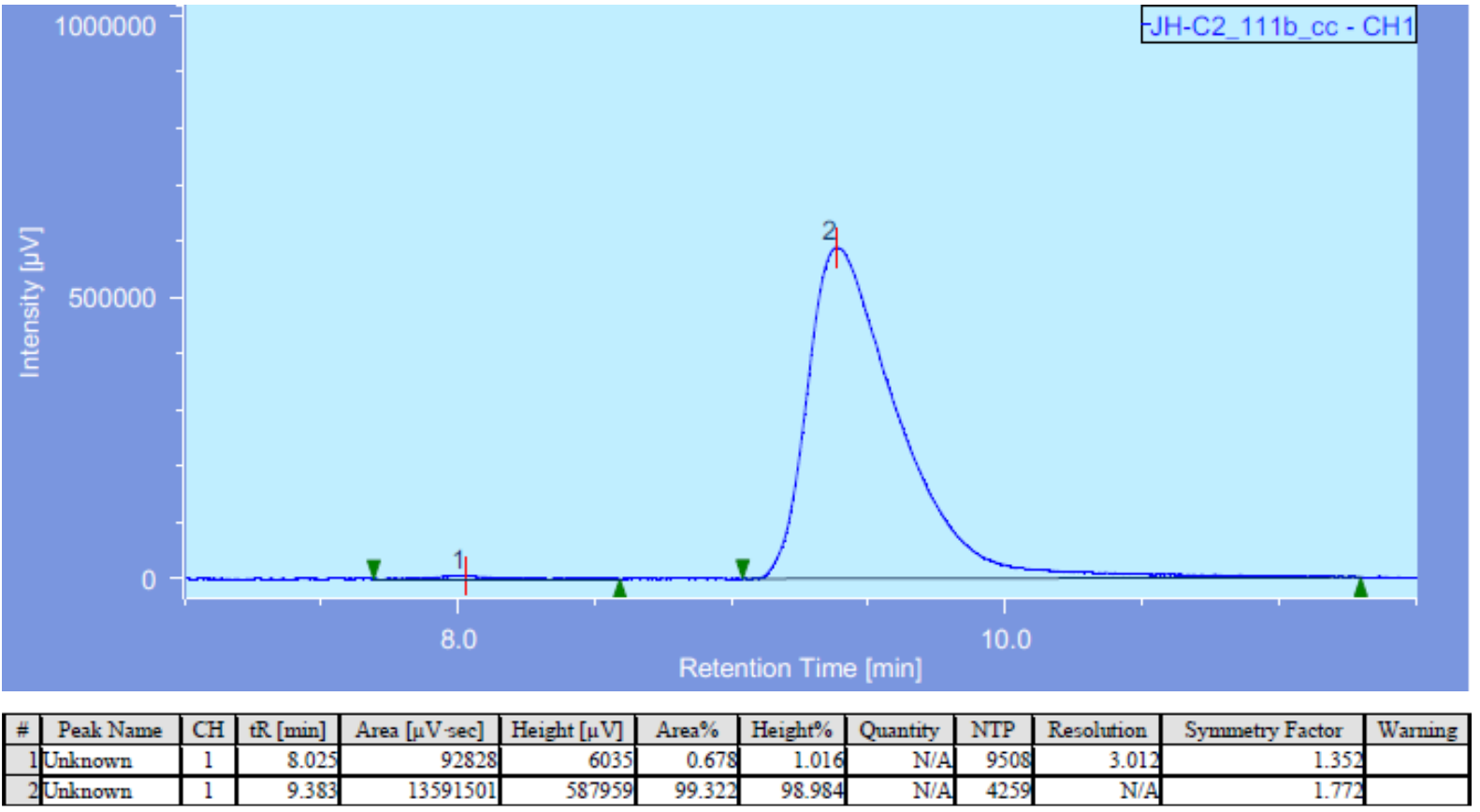

\section{Racemic}

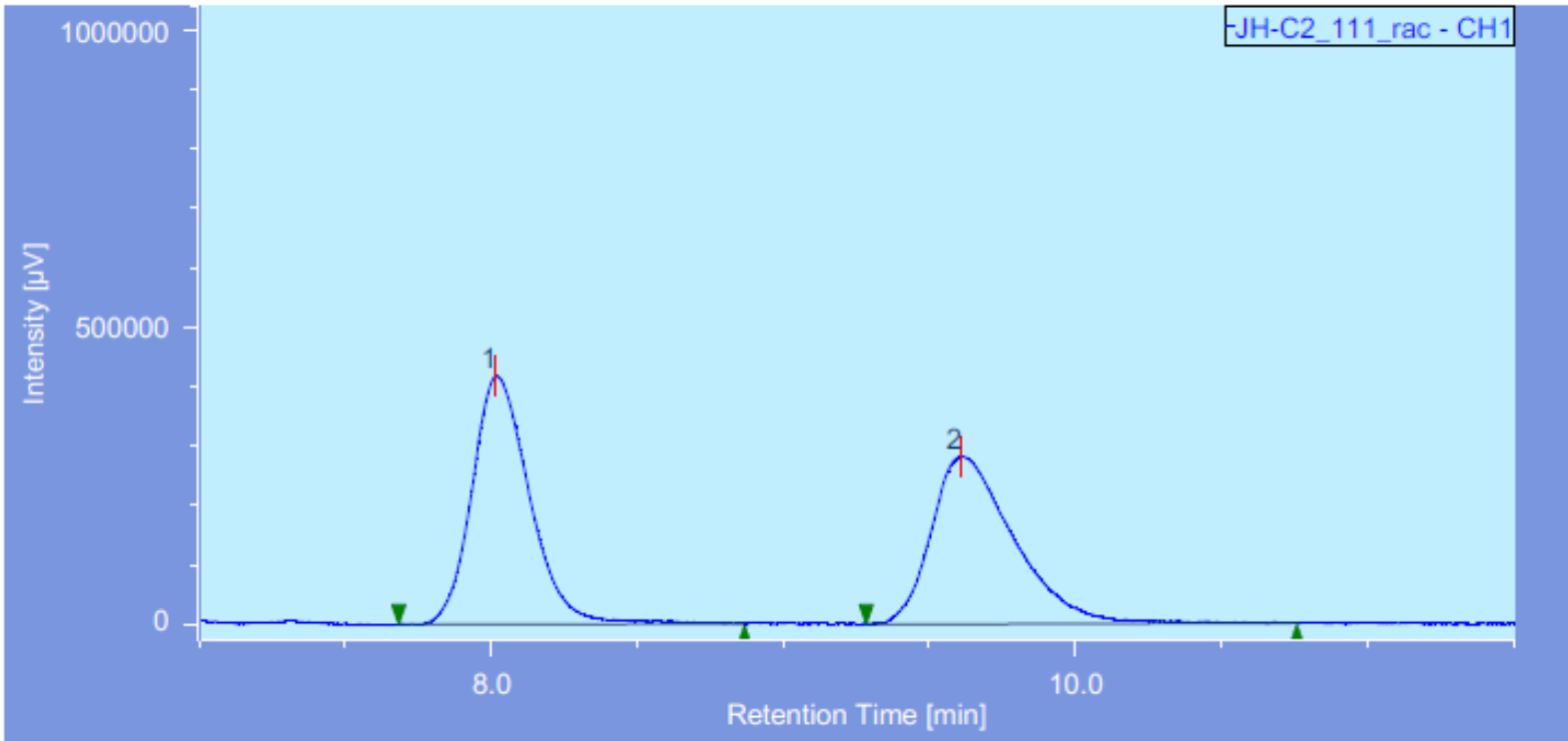

\begin{tabular}{|c|c|c|c|c|c|c|c|c|c|c|c|}
\hline Peak Name & $\mathrm{CH}$ & $t \mathbb{R}[\min ]$ & Area $[\mu \mathrm{V}$-sec $]$ & Height $[\mu \mathrm{V}]$ & Area\% & Height $\%$ & Quantity & NTP & Resolution & Symmetry Factor & Warning \\
\hline 1 Unknown & 1 & 8.01 & 5745958 & 417236 & 50.906 & 59.672 & $\mathrm{~N} / \mathrm{A}$ & 8340 & 3.75 & 1.23 & \\
\hline 2 Unknown & 1 & 9.60 & 5541421 & 281976 & 49.094 & 40.328 & $\mathrm{~N} / \mathrm{A}$ & 5923 & $\mathrm{~N} / \mathrm{A}$ & 1.499 & \\
\hline
\end{tabular}



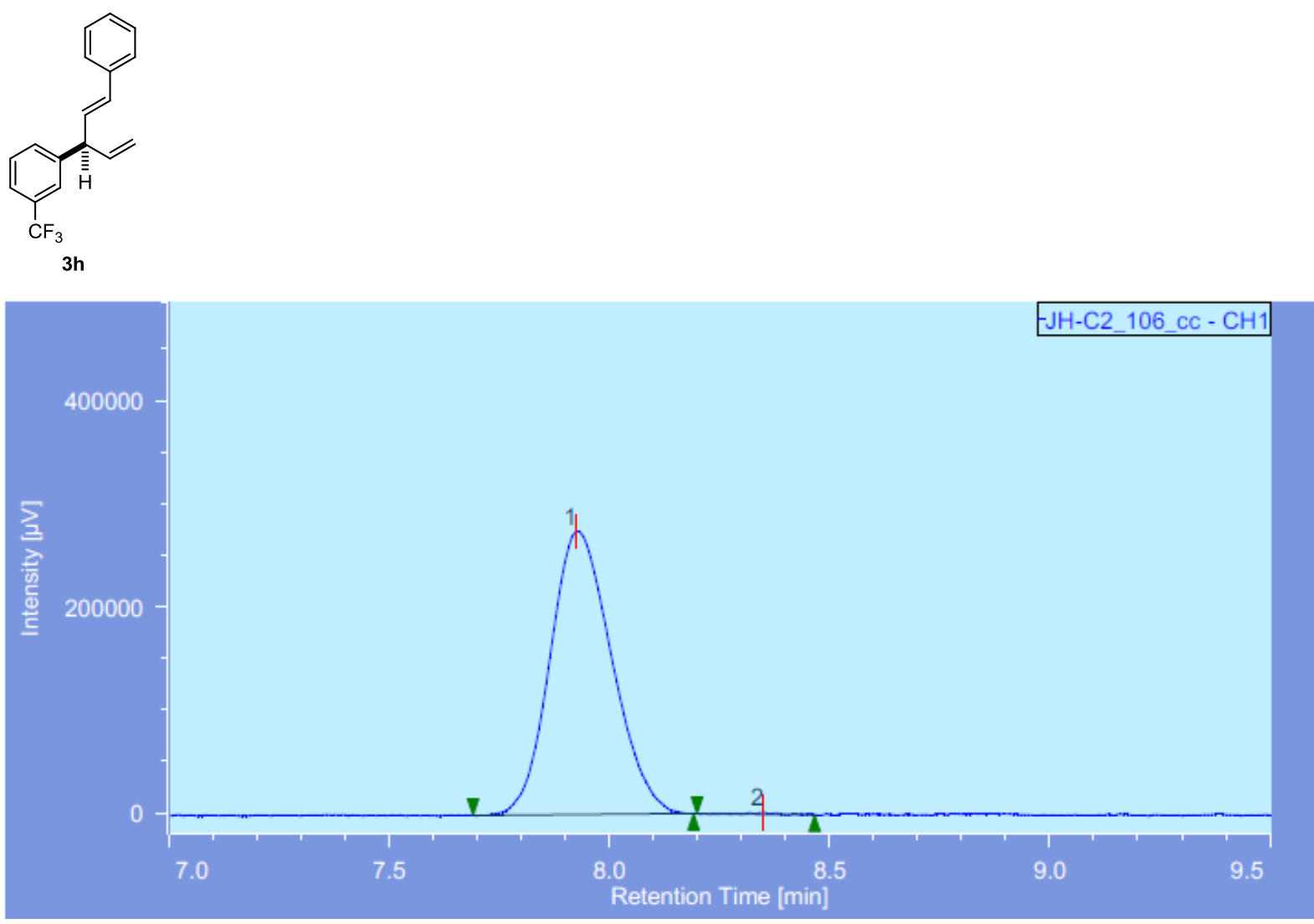

\begin{tabular}{|c|c|c|c|c|c|c|c|c|c|c|c|c|}
\hline$\#$ & Peak Name & $\mathrm{CH}$ & $\mathbb{R}$ [min] & Area $[\mu \mathrm{V} \cdot \mathrm{sec}]$ & Height $[\mu \mathrm{V}]$ & Area\% & Height\% & Quantity & NTP & Resolution & Symmetry Factor & Warning \\
\hline & Unknown & 1 & 7.925 & 2669981 & 274590 & 99.746 & 99.435 & $\mathrm{~N} / \mathrm{A}$ & 15168 & 2.250 & 1.173 & \\
\hline & Unknown & $\overline{1}$ & 8.350 & 6796 & 1561 & 0.254 & 0.565 & N/A & 75678 & $\mathrm{~N} / \mathrm{A}$ & 0.921 & \\
\hline
\end{tabular}

\section{Racemic}

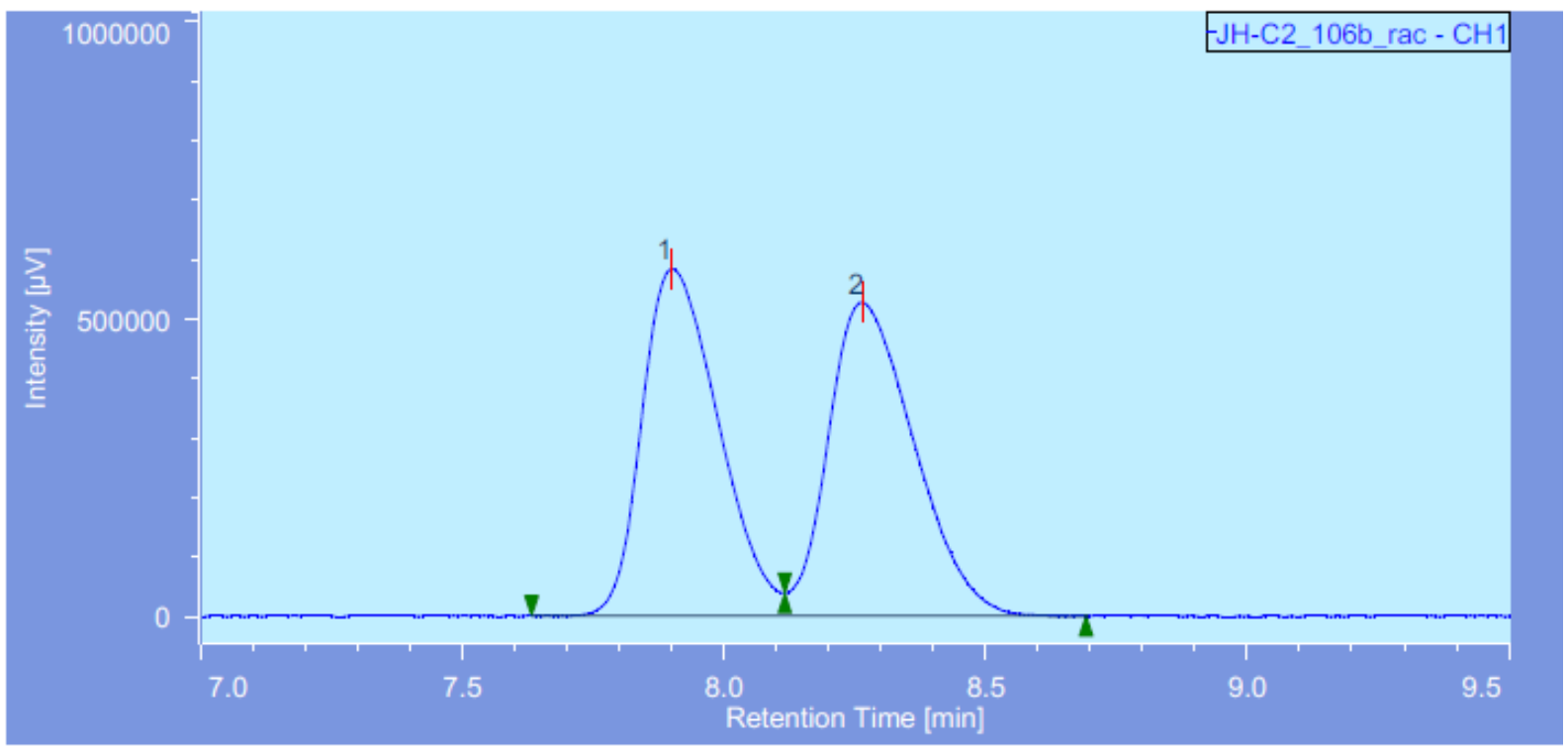

\begin{tabular}{|c|c|c|c|c|c|c|c|c|c|c|c|c|}
\hline$\#$ & Peak Name & $\mathrm{CH}$ & $t \mathbb{R}[\min ]$ & Area $[\mu \mathrm{V} \cdot \mathrm{sec}]$ & Height $[\mu \mathrm{V}]$ & Area $\%$ & Height $\%$ & Quantity & NTP & Resolution & Symmetry Factor & Warning \\
\hline & Unknown & 1 & 7.900 & 5910597 & 583190 & 49.633 & 52.545 & $\mathrm{~N} / \mathrm{A}$ & 13487 & 1.280 & $\mathrm{~N} / \mathrm{A}$ & \\
\hline 2 & Unknown & 1 & 8.267 & 5998001 & 526703 & 50.367 & 47.455 & $\mathrm{~N} / \mathrm{A}$ & 11951 & $\mathrm{~N} / \mathrm{A}$ & $\mathrm{N} / \mathrm{A}$ & \\
\hline
\end{tabular}



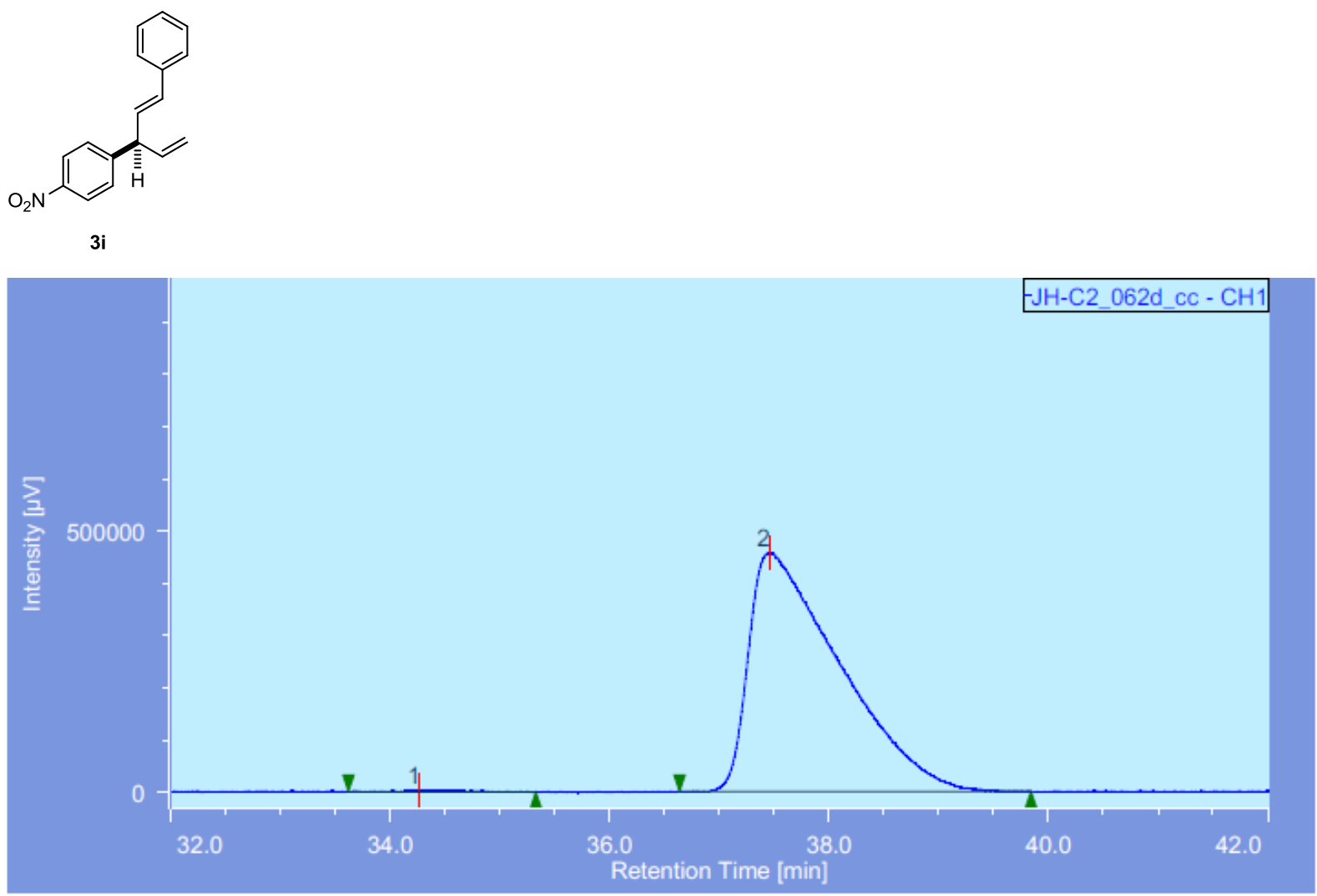

\begin{tabular}{|c|c|c|c|c|c|c|c|c|c|c|c|}
\hline$\#$ \# Peak Name & $\mathrm{CH}$ & $t R[\min ]$ & Area $[\mu \mathrm{V} \cdot \mathrm{sec}]$ & \begin{tabular}{|l|} 
Height $[\mu \mathrm{V}]$ \\
\end{tabular} & Area\% & Height\% & \begin{tabular}{|l|} 
Quantity \\
\end{tabular} & NIP & \begin{tabular}{|l|l|l} 
Resolution \\
\end{tabular} & Symmetry Factor & Warning \\
\hline Unknown & 1 & 34.278 & 85484 & 3030 & 0.32 & 0.659 & $\mathrm{~N} / \mathrm{A}$ & 28683 & 2.736 & 1.112 & \\
\hline Unknown & 1 & $37.45 \mathrm{~s}$ & 26236685 & 456724 & 99.67 & 99.341. & N/A & 9673 & N/A & 2.499 & \\
\hline
\end{tabular}

\section{Racemic}

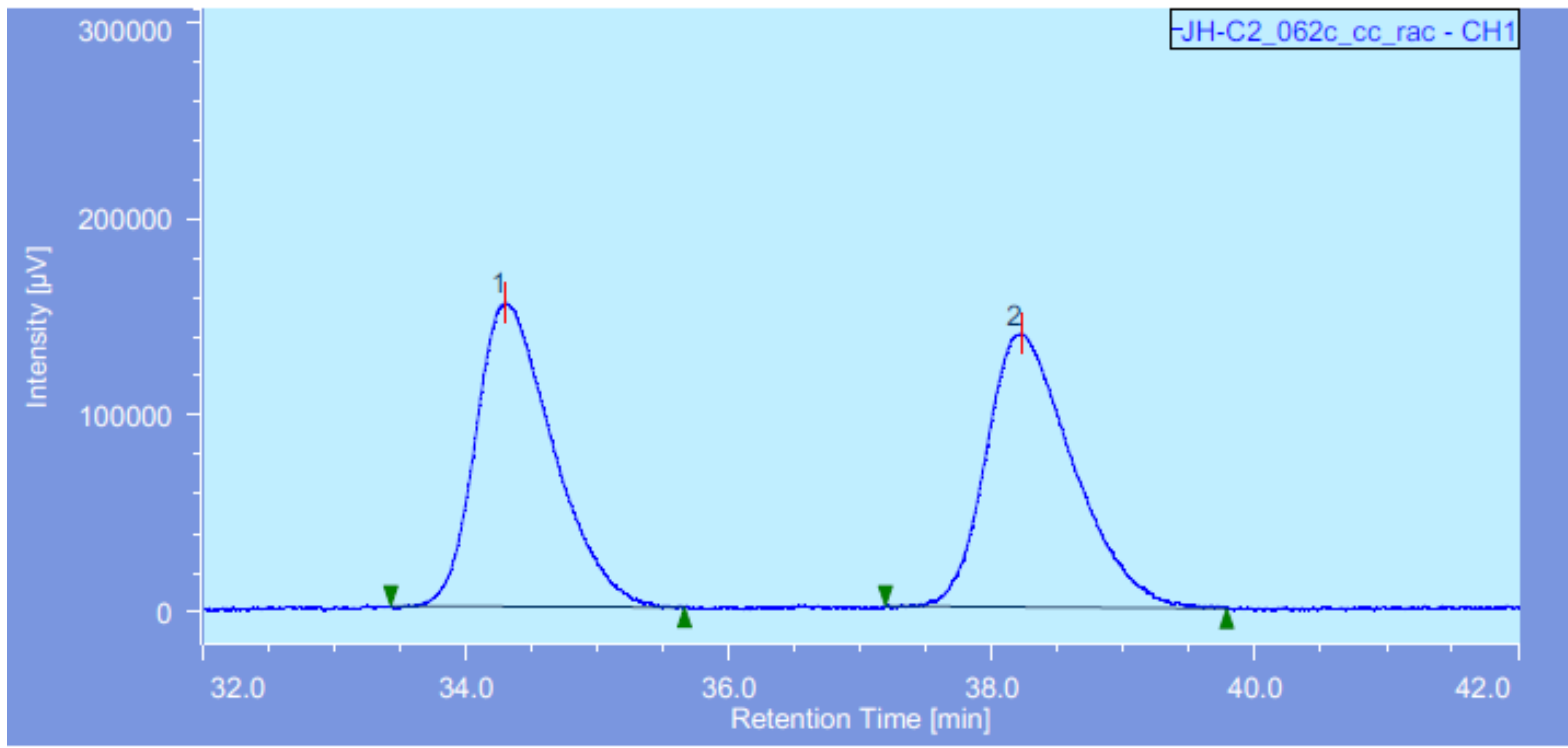

\begin{tabular}{|c|c|c|c|c|c|c|c|c|c|c|c|c|}
\hline$\#$ & Peak Name & $\mathrm{CH}$ & $t R[\min ]$ & Area $[\mu \mathrm{V}-s e c]$ & Height $[\mu \mathrm{V}]$ & Area\% & Height $\%$ & Quantity & NTP & Resolution & Symmetry Factor & \begin{tabular}{|l|} 
Warning \\
\end{tabular} \\
\hline & Unknown & 1 & $34.30 \mathrm{~s}$ & 6284877 & 154865 & 49.659 & 52.660 & $\mathrm{~N} / \mathrm{A}$ & 16820 & 3.493 & 1.388 & \\
\hline & Unknown & 1 & 38.225 & 6371316 & 139221 & 50.341 & 47.340 & $\mathrm{~N} / \mathrm{A}$ & 16493 & $\mathrm{~N} / \mathrm{A}$ & 1.355 & \\
\hline
\end{tabular}




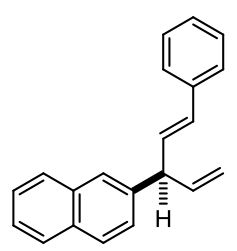

3j

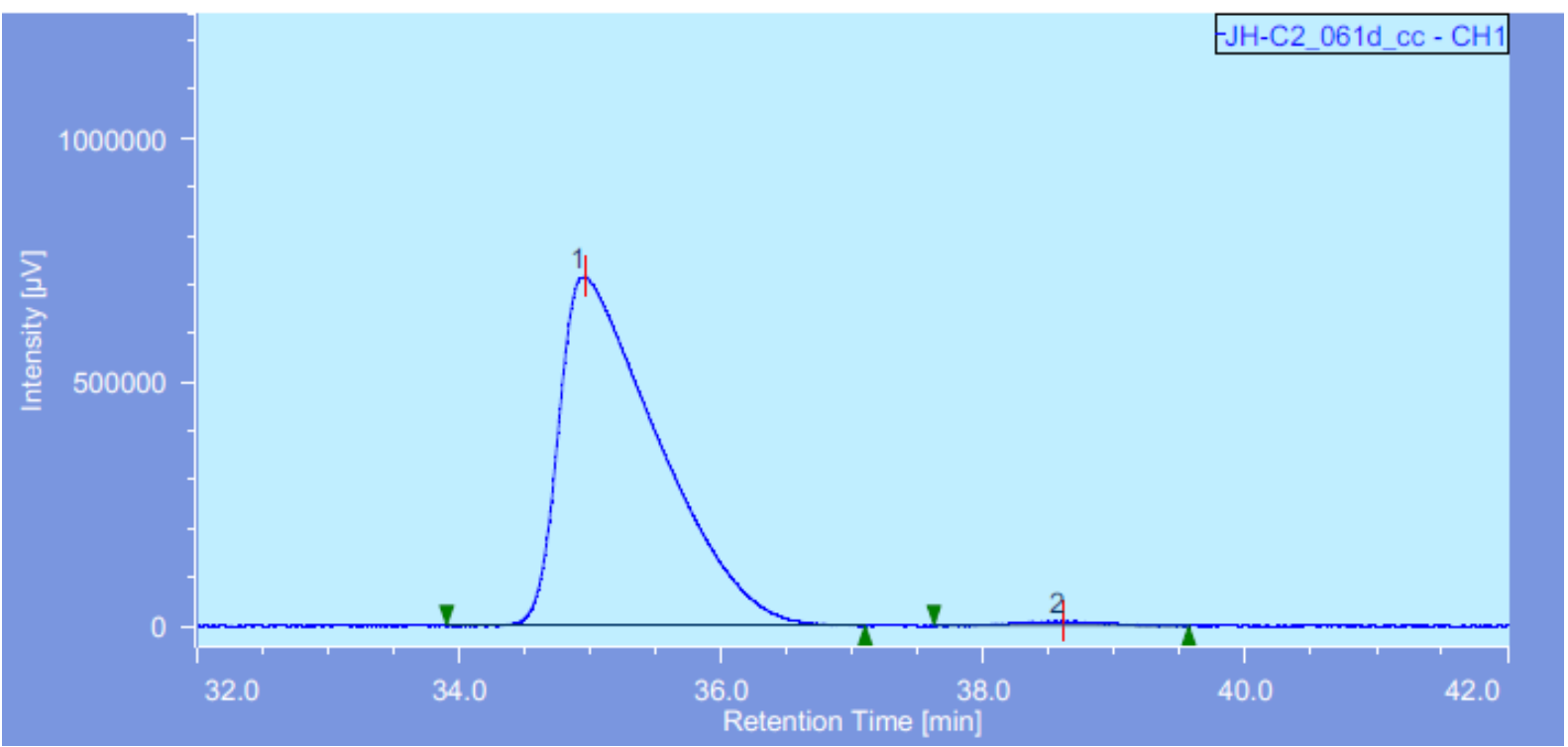

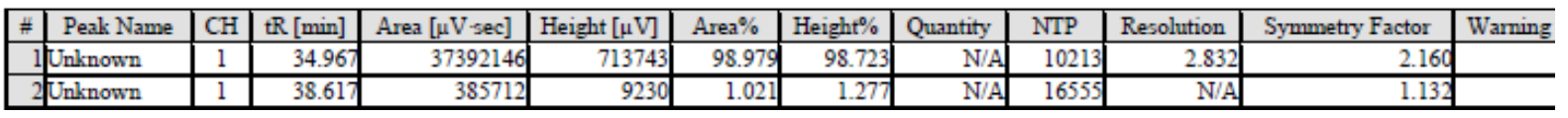

\section{Racemic}

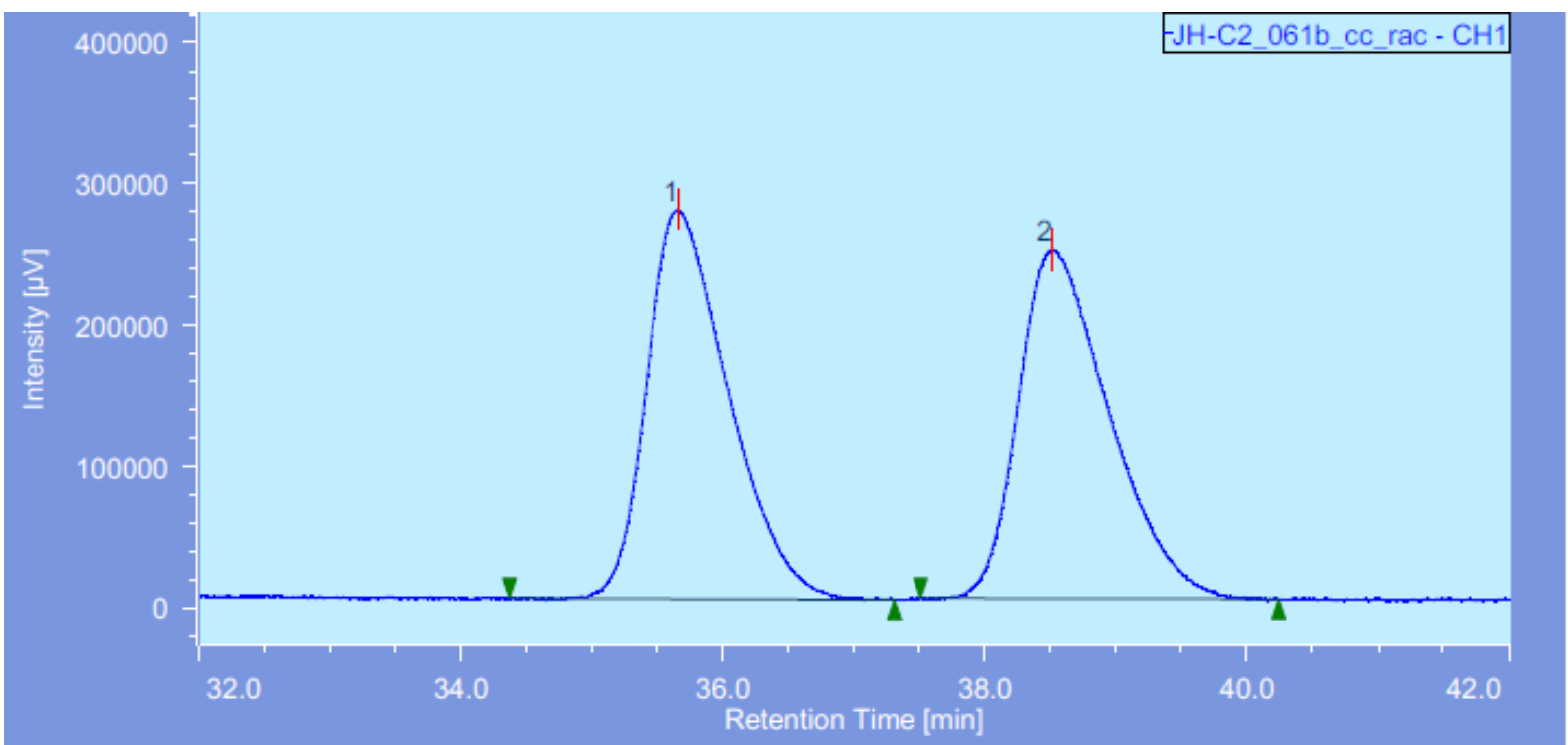

\begin{tabular}{|c|c|c|c|c|c|c|c|c|c|c|c|c|}
\hline$\#$ & Peak Name & $\mathrm{CH}$ & $t \mathbb{R}$ [min] & Area $[\mu \mathrm{V}-\mathrm{sec}]$ & Height $[\mu \mathrm{V}]$ & Area\% & Height $\%$ & Quantity & NTP & Resolution & Symmetry Factor & Warning \\
\hline & Unknown & 1 & 35.65 & 11927299 & 275213 & 50.229 & 52.701 & $\mathrm{~N} / \mathrm{A}$ & 15867 & 2.398 & 1.401 & \\
\hline & Unknown & $\overline{1}$ & 38.50 & 11818766 & 247003 & 49.771 & 47.299 & $\mathrm{~N} / \mathrm{A}$ & 15165 & $\mathrm{N} / \mathrm{A}$ & 1.477 & \\
\hline
\end{tabular}



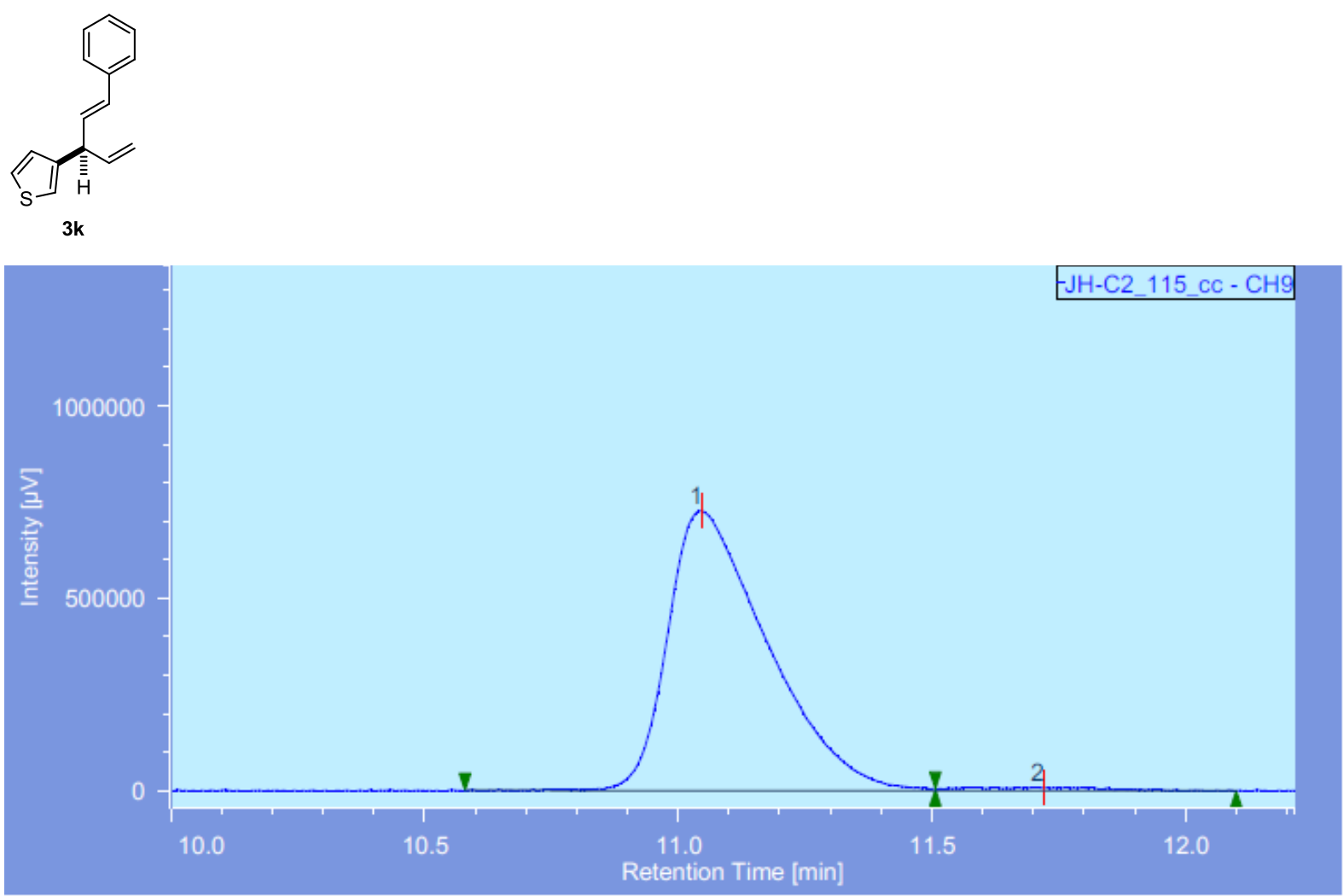

\begin{tabular}{|c|c|c|c|c|c|c|c|c|c|c|c|}
\hline \begin{tabular}{l|l}
$\#$ & Peak Name
\end{tabular} & $\mathrm{CH}$ & $\mathbb{t R}[\min ]$ & Area $[\mu \mathrm{V} \cdot \mathrm{sec}]$ & \begin{tabular}{|l|} 
Height $[\mu \mathrm{V}]$ \\
\end{tabular} & Area\% & Height\% & \begin{tabular}{|l|} 
Quantity \\
\end{tabular} & NTP & \begin{tabular}{|l|l|l} 
Resolution \\
\end{tabular} & Symmetry Factor & Warning \\
\hline Unknown & 9 & 11.047 & 9916670 & 726591 & 98.59 & 98.89 & $\mathrm{~N} / \mathrm{A}$ & 15503 & N/A & 1.63 & \\
\hline Unknown & 9 & 11.720 & 140974 & 8140 & 1.40 & 1.10 & N/A & $\mathrm{N} / \mathrm{A}$ & N/A & $\overline{N / A}$ & \\
\hline
\end{tabular}

\section{Racemic}

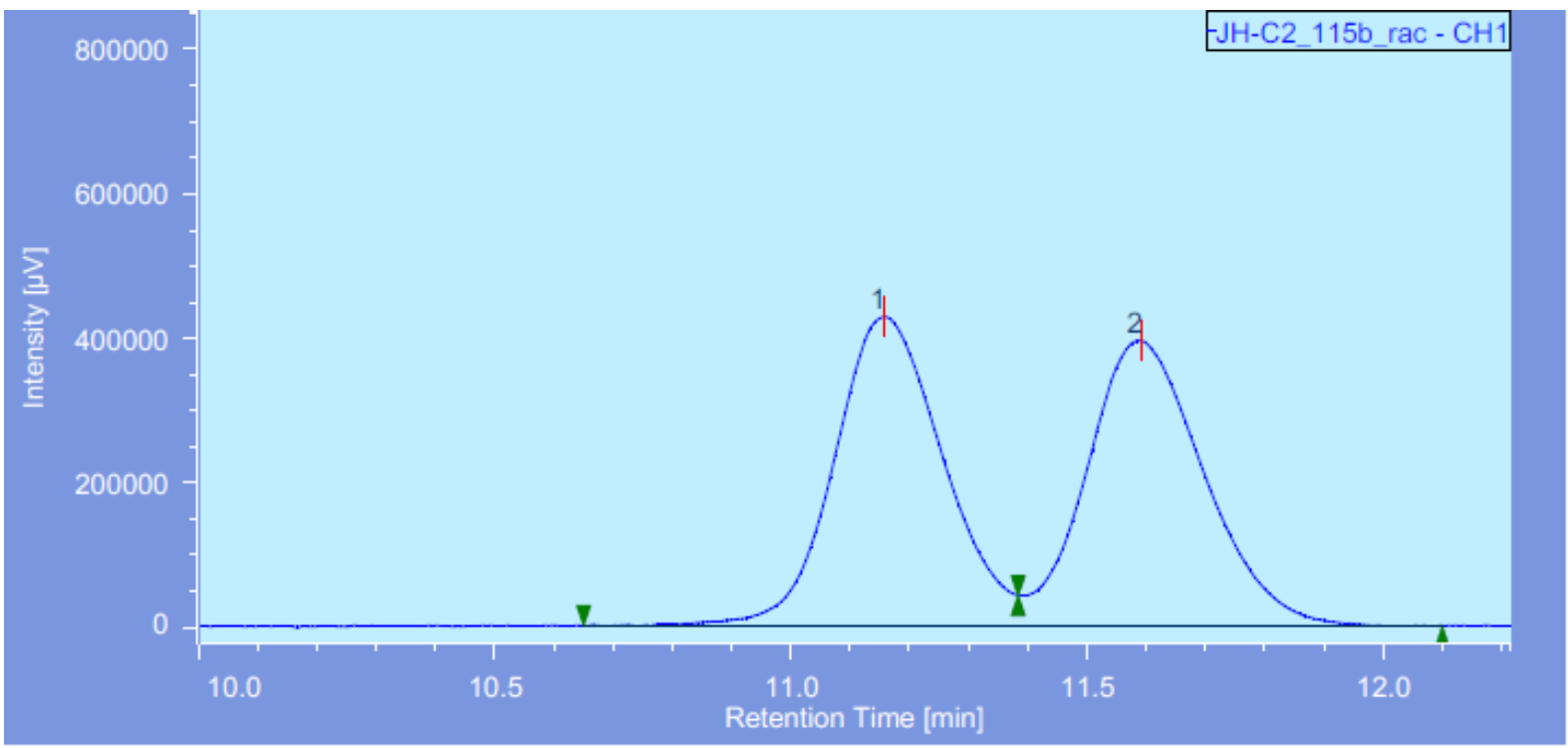

\begin{tabular}{|c|c|c|c|c|c|c|c|c|c|c|c|}
\hline \begin{tabular}{l|l} 
Peak Name \\
\end{tabular} & $\mathrm{CH}$ & $t \mathbb{R}[\min ]$ & Area $[\mu \mathrm{V}$-sec] & Height $[\mu \mathrm{V}]$ & Area\% & Height $\%$ & Quantity & NTP & Resolution & Symmetry Factor & Warning \\
\hline 1 Unknown & 1 & $11.15 \mathrm{~s}$ & 5408292 & 427806 & 50.031 & 52.038 & N/A & 18188 & 1.262 & $\mathrm{~N} / \mathrm{A}$ & \\
\hline Unknown & $\mathrm{l}$ & 11.592 & 5401624 & 394305 & 49.969 & 47.962 & N/A & 16823 & $\mathrm{~N} / \mathrm{A}$ & $\mathrm{N} / \mathrm{A}$ & \\
\hline
\end{tabular}



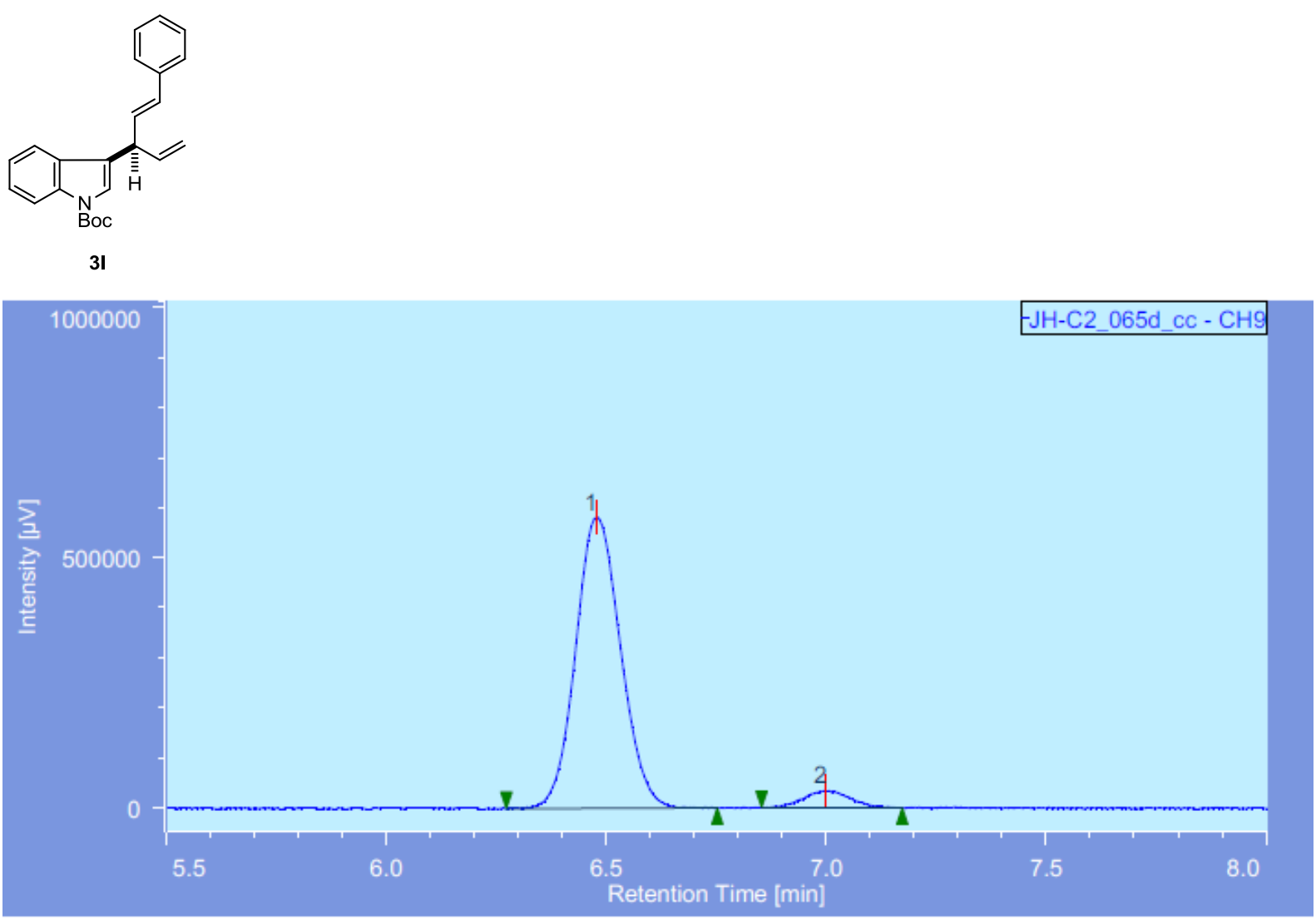

\begin{tabular}{|c|c|c|c|c|c|c|c|c|c|c|c|c|}
\hline$\#$ & Peak Name & $\mathrm{CH}$ & $\mathbb{t R}[\min ]$ & Area $[\mu \mathrm{V}-\sec ]$ & Height $[\mu \mathrm{V}]$ & Area\% & Height\% & Quantity & NTP & Resolution & Symmetry Factor & Warning \\
\hline & Unknown & 9 & $6.48 \mathrm{~d}$ & 4119120 & 581939 & 94.294 & 94.459 & $\mathrm{~N} / \mathrm{A}$ & 19438 & 2.755 & 1.086 & \\
\hline & Unknown & 9 & 7.000 & 249247 & 34135 & 5.706 & 5.541 & $\mathrm{~N} / \mathrm{A}$ & 21134 & N/A & 1.034 & \\
\hline
\end{tabular}

\section{Racemic}

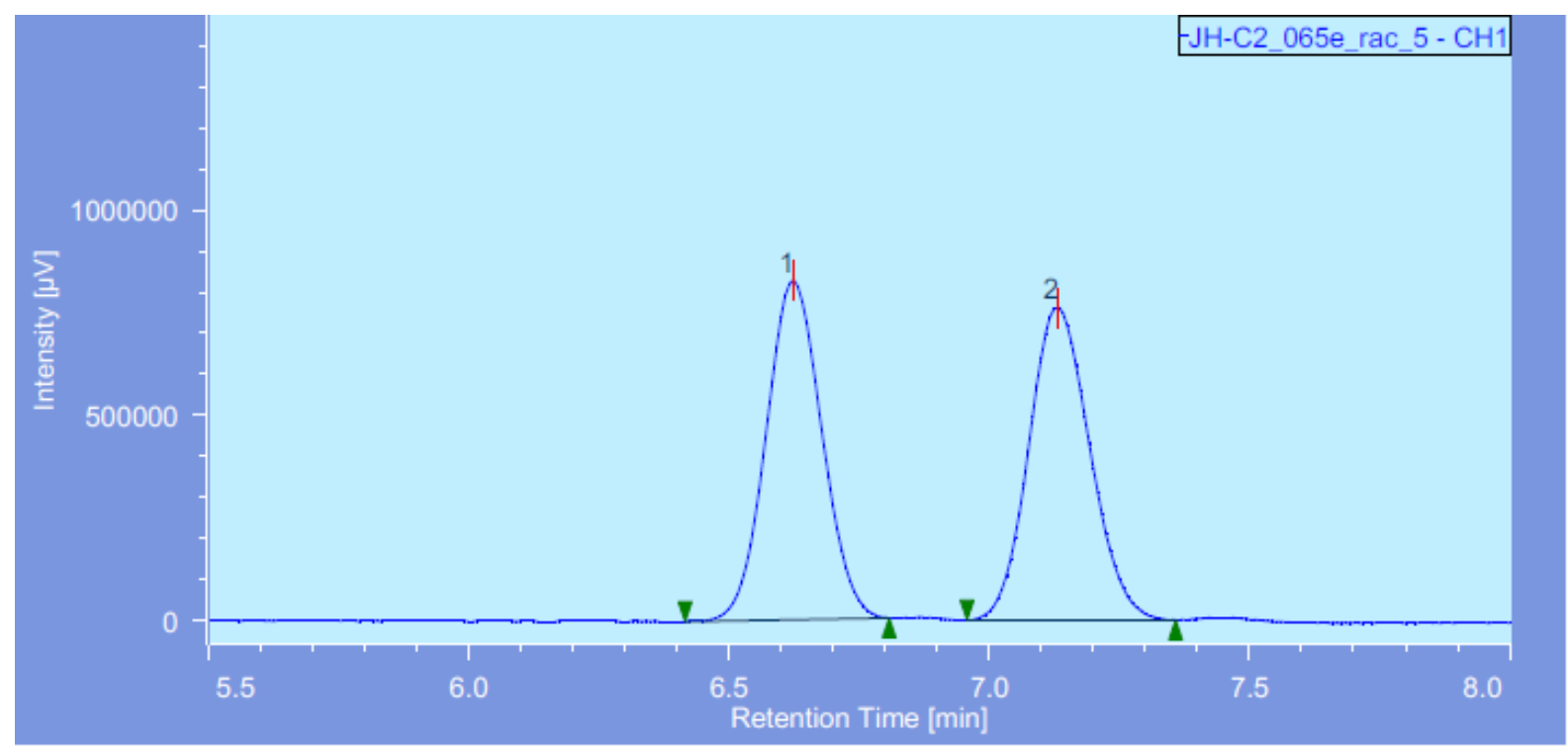

\begin{tabular}{|c|c|c|c|c|c|c|c|c|c|c|c|}
\hline Peak Name & $\mathrm{CH}$ & $t \mathbb{R}[\min ]$ & Area $[\mu \mathrm{V} \cdot \mathrm{sec}]$ & Height $[\mu \mathrm{V}]$ & Area\% & Height\% & Quantity & NTP & Resolution & Symmetry Factor & Warning \\
\hline Unknown & 1 & 6.625 & 6162588 & 827217 & 49.875 & 52.082 & $\mathrm{~N} / \mathrm{t}$ & 17950 & 2.465 & 1.04 & \\
\hline Unknown & $\mathrm{l}$ & 7.133 & 6193417 & 761066 & 50.125 & 47.918 & $\overline{\mathrm{N} / \mathrm{t}}$ & 17485 & $\mathrm{~N} / \mathrm{A}$ & 1.09 & \\
\hline
\end{tabular}



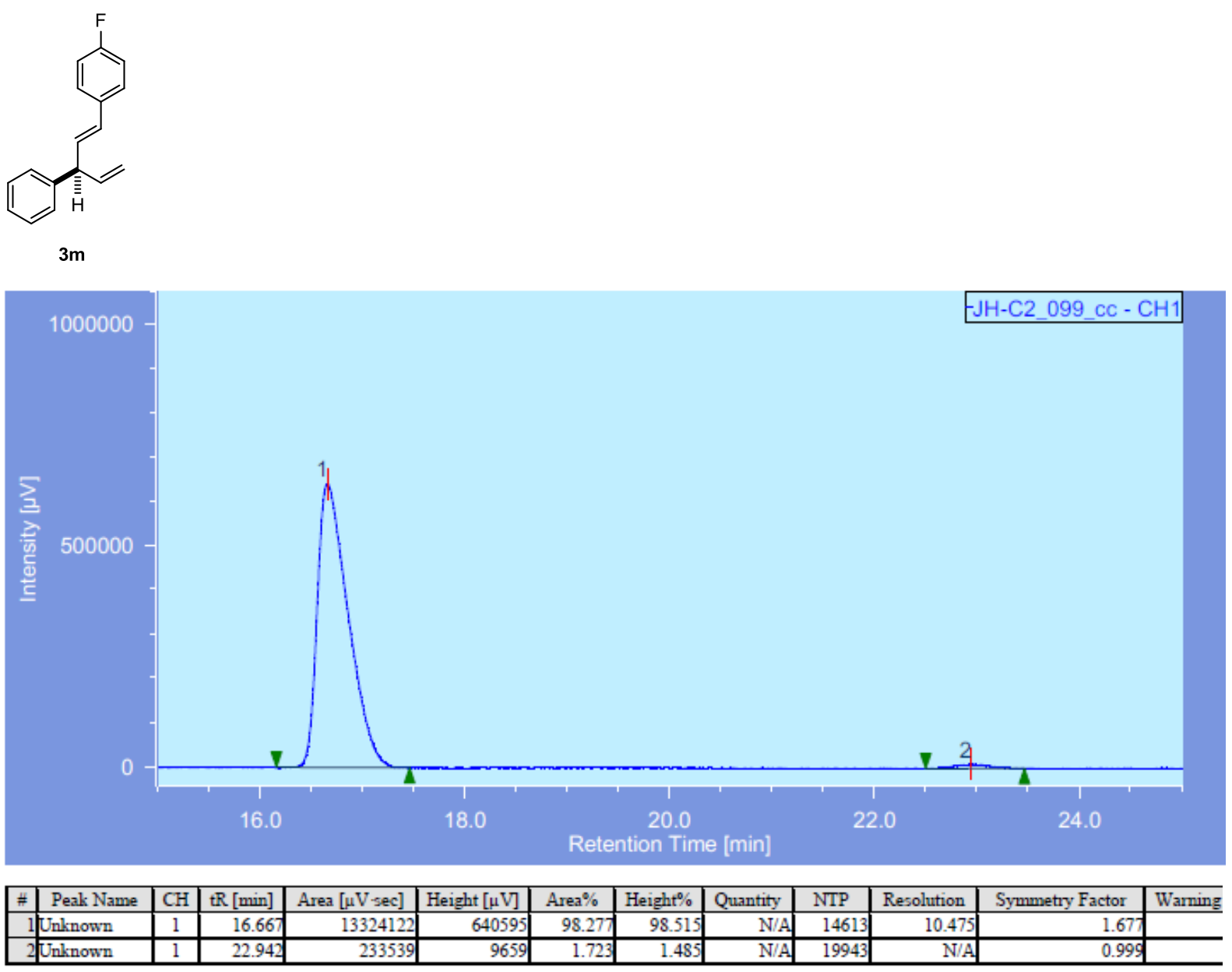

\section{Racemic}

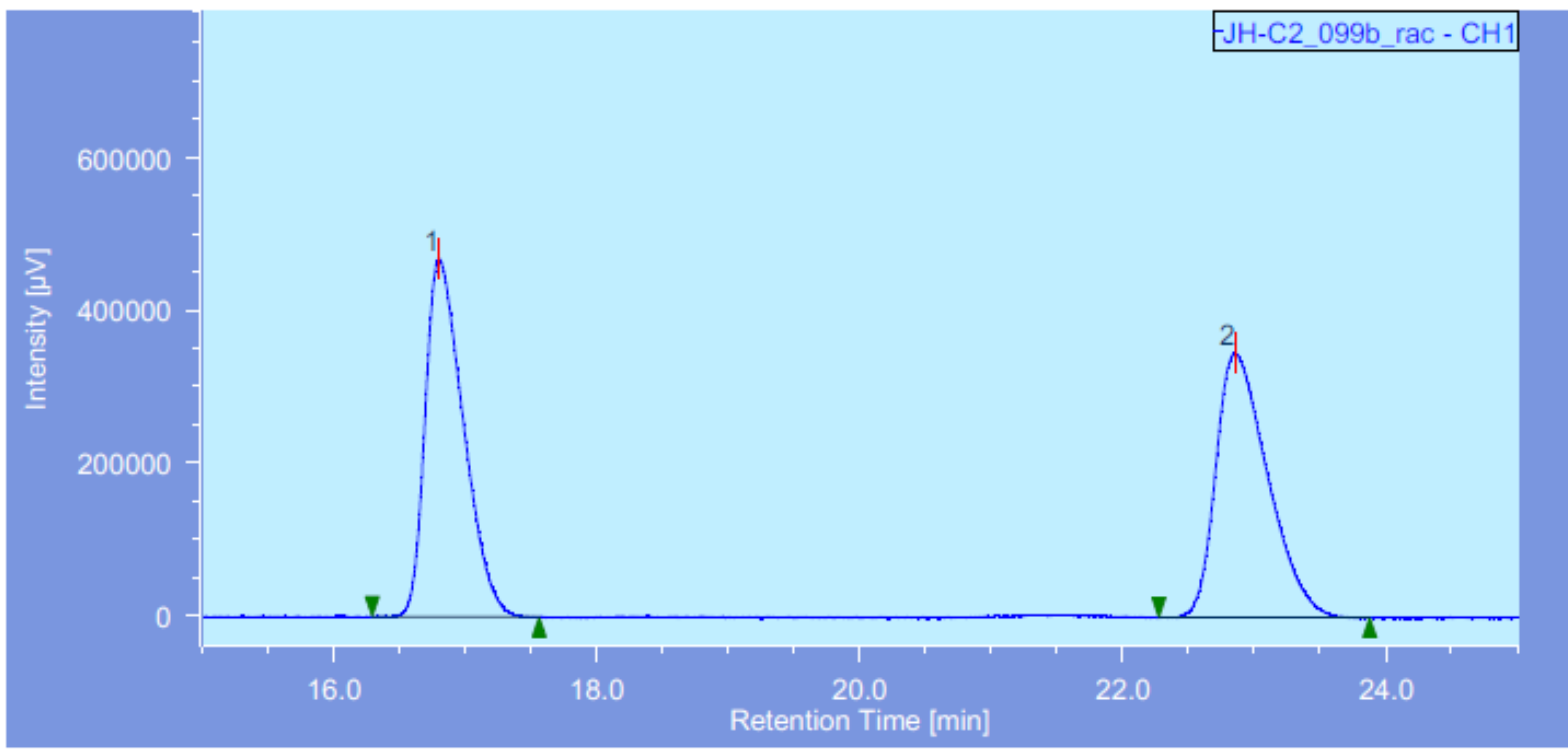

\begin{tabular}{|c|c|c|c|c|c|c|c|c|c|c|c|c|}
\hline 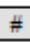 & Peak Name & $\mathrm{CH}$ & $t \mathbb{R}$ [min] & Area $[\mu \mathrm{V}$-sec] & Height $[\mu \mathrm{V}]$ & Area $\%$ & Height $\%$ & Quantity & NTP & Resolution & Symmetry Factor & Warning \\
\hline & Unknown & 1 & 16.808 & 9418534 & 467792 & 49.882 & 57.488 & N/A & 15876 & 9.637 & 1.487 & \\
\hline & Unknown & 1 & 22.850 & 9463007 & 345936 & 50.118 & 42.512 & N/A & 15956 & $\mathrm{~N} / \mathrm{A}$ & $1.48 \mathrm{~S}$ & \\
\hline
\end{tabular}




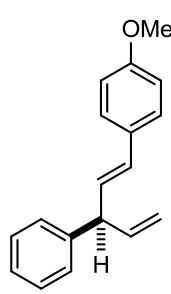

3n

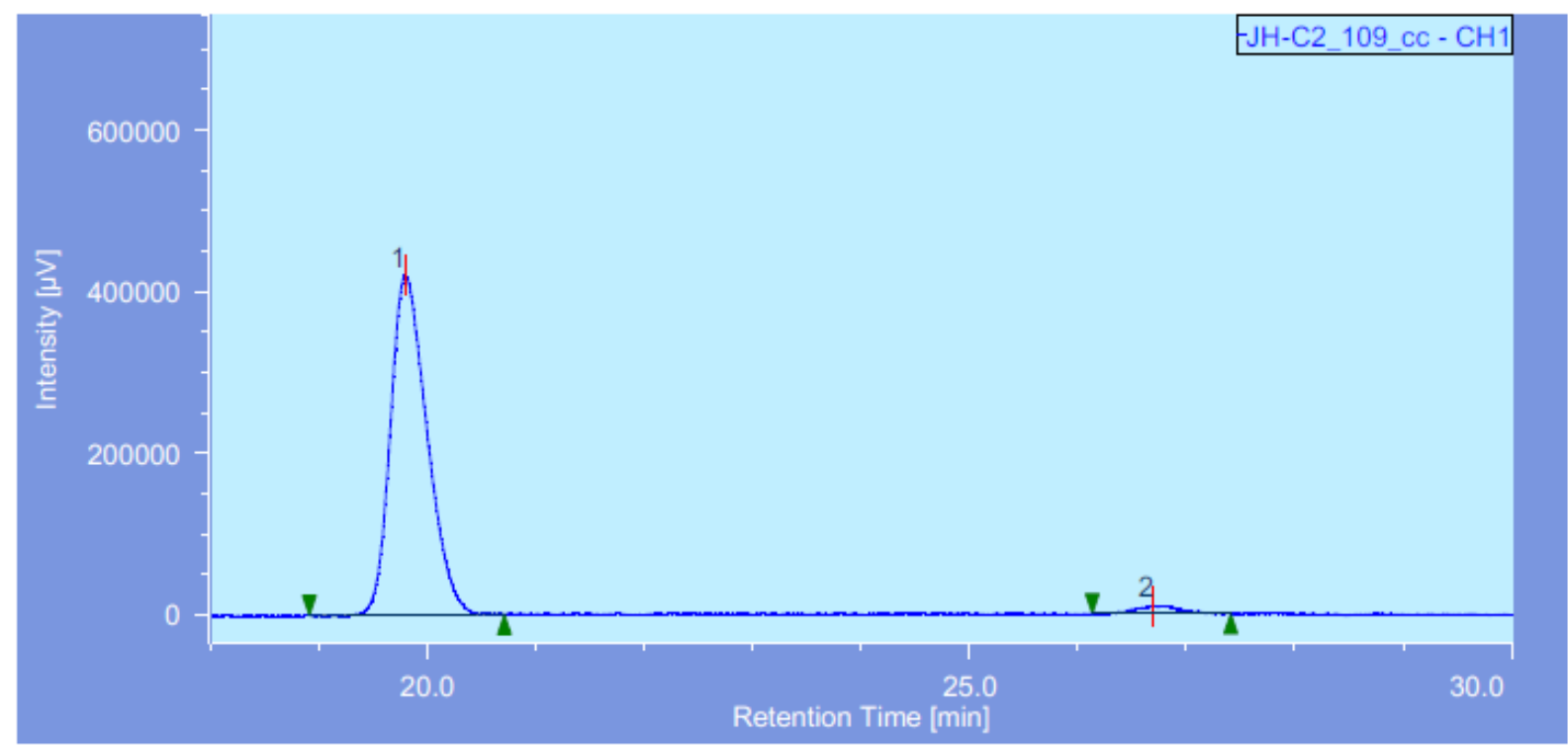

\begin{tabular}{|c|c|c|c|c|c|c|c|c|c|c|c|c|}
\hline$\#$ & Peak Name & $\mathrm{CH}$ & $t \mathbb{R}[\min ]$ & Area [ $[\mathrm{V}$-sec] & Height $[\mu \mathrm{V}]$ & Area $\%$ & Height $\%$ & Quantity & NIP & Resolution & Symmetry Factor & \begin{tabular}{|l|} 
Warming \\
\end{tabular} \\
\hline & Unknown & 1 & 19.800 & 9829798 & 419939 & 97.335 & 97.708 & $\mathrm{~N} / \mathrm{A}$ & 16614 & 10.252 & 1.30 t & \\
\hline & Unknown & 1 & 26.692 & 269131 & 9851 & 2.665 & 2.292 & N/A & 21181 & N/A & 1.404 & \\
\hline
\end{tabular}

\section{Racemic}

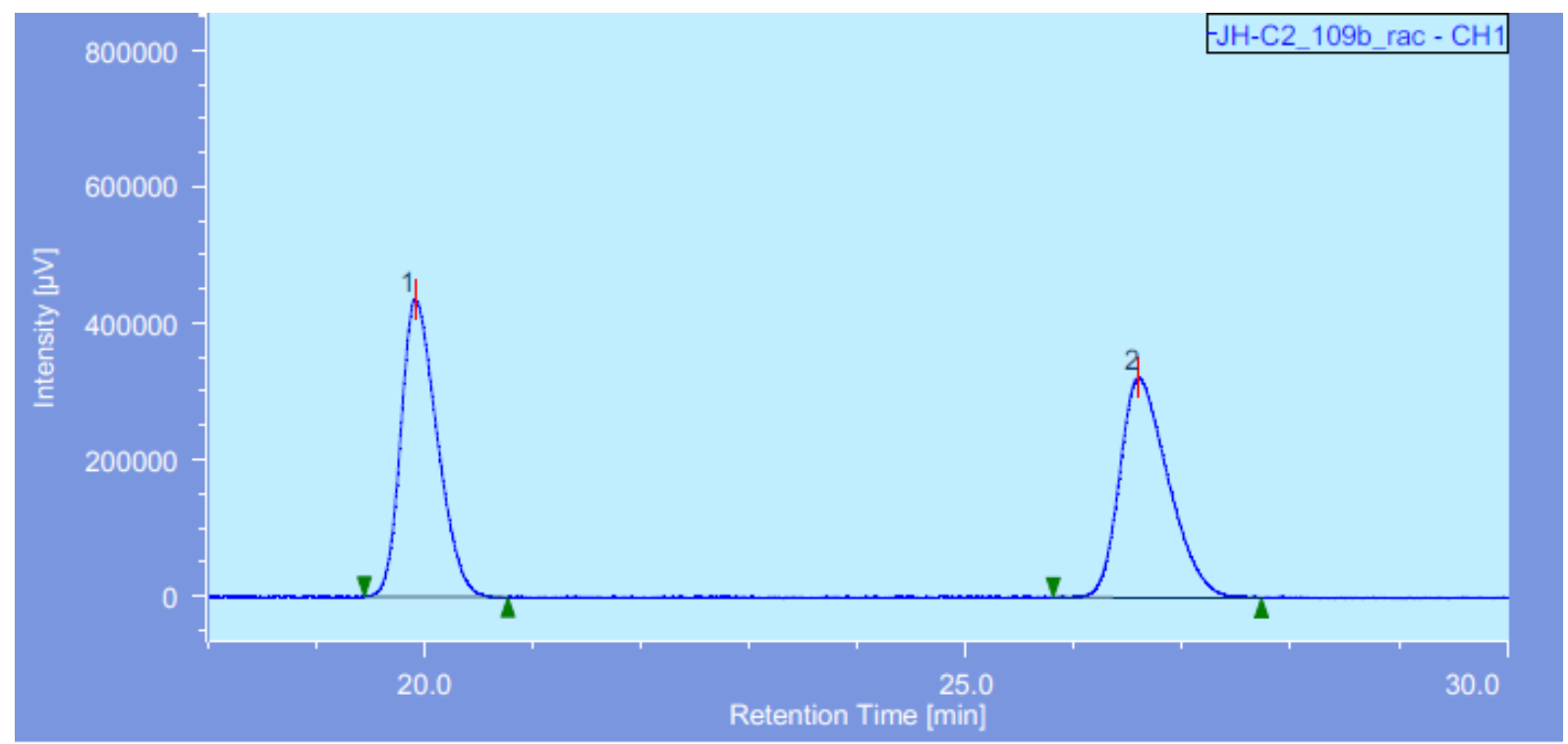

\begin{tabular}{|c|c|c|r|r|r|r|r|r|r|r|r|r}
\hline$\#$ & Pealk Name & $\mathrm{CH}$ & $\mathrm{tR}[\mathrm{min}]$ & Area $[\mu \mathrm{V}$-sec] & Height $[\mu \mathrm{V}]$ & Area\% & Height\% & Quantity & NTP & Resolution & Symmetry Factor & Warning \\
\hline 1 & Unknown & 1 & 19.917 & 10123794 & 436159 & 50.196 & 57.558 & N/A & 16958 & 9.366 & 1.322 & \\
\hline 2 Unknnown & 1 & 26.600 & 10044758 & 321609 & 49.804 & 42.442 & N/A & 16873 & N/A & & 1.352 & \\
\hline
\end{tabular}


OMe

30

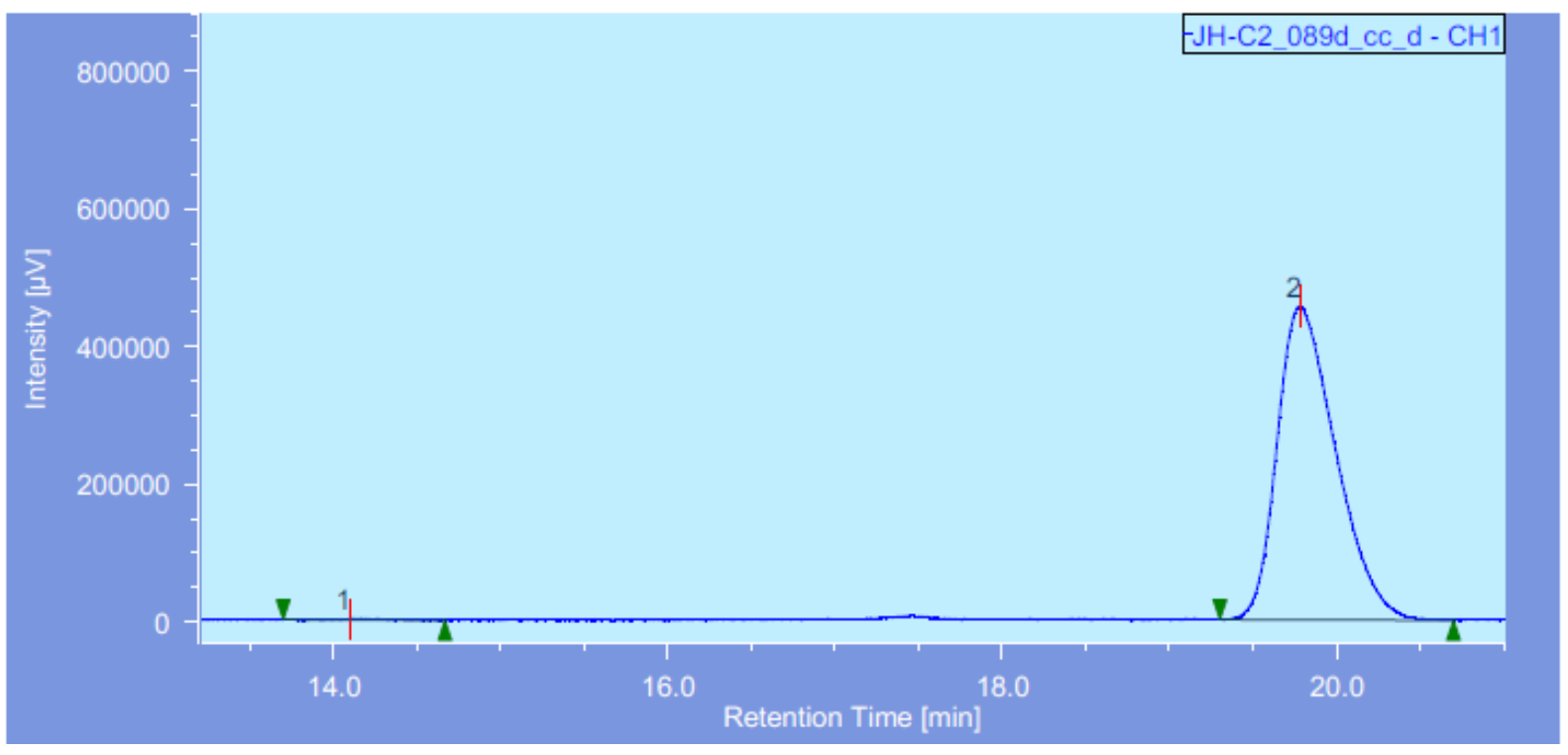

\begin{tabular}{|c|c|c|c|c|c|c|c|c|c|c|c|c|}
\hline$\#$ & Peak Name & $\mathrm{CH}$ & $\mathbb{t R}[\min ]$ & Area $[\mu \mathrm{V}-\mathrm{sec}]$ & Height $[\mu \mathrm{V}]$ & Area\% & Height $\%$ & Quantity & NTP & Resolution & Symmetry Factor & Warning \\
\hline & Unknown & 1 & $14.10 \mathrm{~d}$ & 45463 & 2006 & 0.409 & 0.438 & $\mathrm{~N} / \mathrm{A}$ & 4416 & 7.655 & 1.218 & \\
\hline & Unknown & $\bar{l}$ & 19.783 & 11070492 & 455479 & 99.591 & 99.562 & $\mathrm{~N} / \mathrm{A}$ & 15280 & N/A- & 1.388 & \\
\hline
\end{tabular}

\section{Racemic}

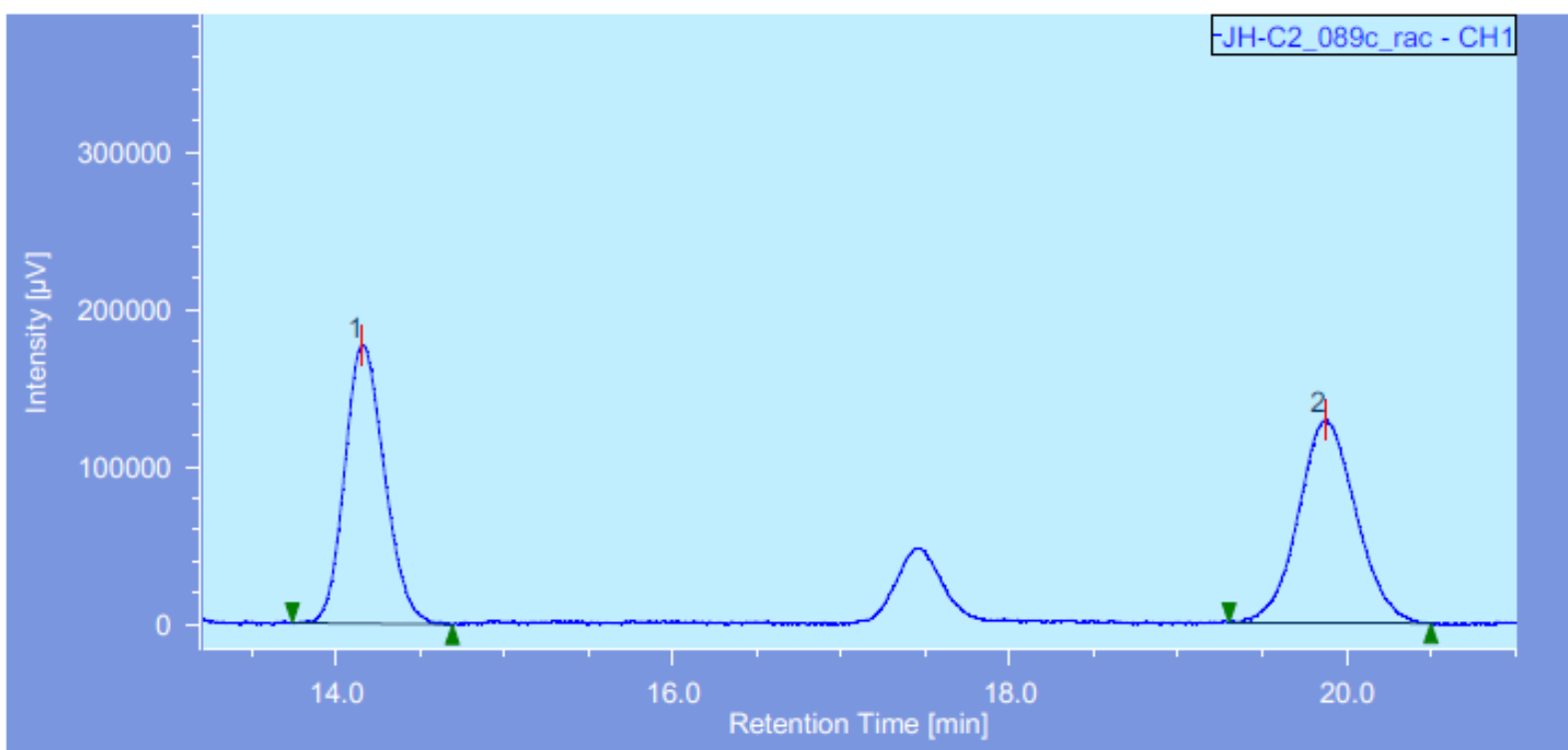

\begin{tabular}{|c|c|c|c|c|c|c|c|c|c|c|c|c|}
\hline$\#$ & Peak Name & $\mathrm{CH}$ & $t \mathbb{R}[\min ]$ & Area $[\mu \mathrm{V}-\sec ]$ & Height $[\mu \mathrm{V}]$ & Area $\%$ & Height\% & Quantity & NTP & Resolution & Symmetry Factor & Warning \\
\hline & Unknown & 1 & $14.15 \mathrm{~g}$ & 2901194 & 176613 & 48.554 & 57.791 & $\mathrm{~N} / \mathrm{A}$ & 17289 & 10.914 & 1.157 & \\
\hline & Unknown & $\overline{1}$ & 19.875 & 3074012 & 128994 & 51.446 & 42.209 & $\mathrm{~N} / \mathrm{A}$ & 16461 & $\mathrm{~N} / \mathrm{A}$ & 1.053 & \\
\hline
\end{tabular}



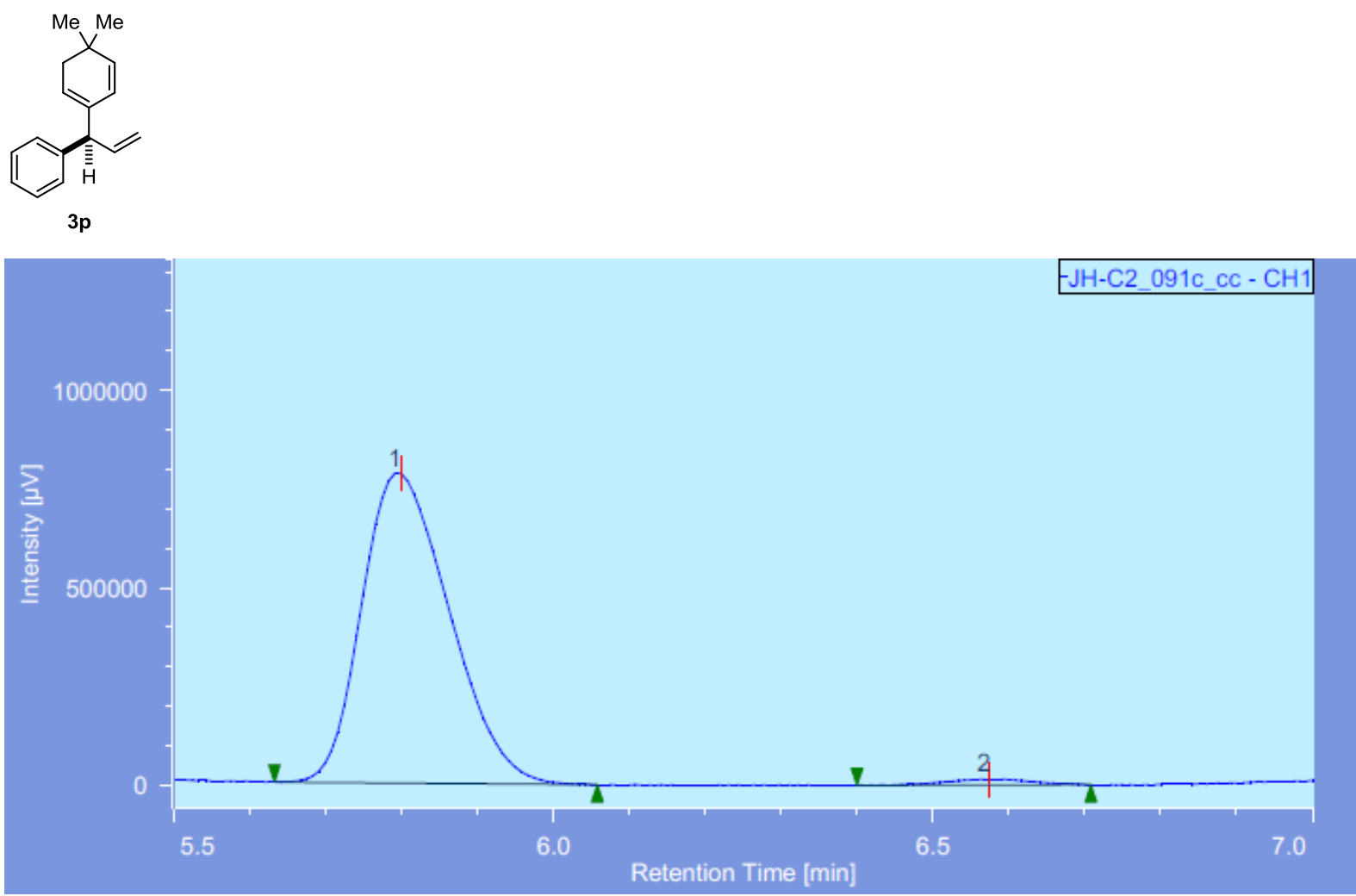

\begin{tabular}{|c|c|c|c|c|c|c|c|c|c|c|c|}
\hline \begin{tabular}{l|l}
$\#$ Peak Name \\
\end{tabular} & $\mathrm{CH}$ & $\mathbb{t R}[\min ]$ & Area $[\mu \mathrm{V} \cdot \mathrm{sec}]$ & Height $[\mu \mathrm{V}]$ & Area\% & Height\% & Quantity & NIP & Resolution & Symmetry Factor & Warning \\
\hline Unknown & 1 & 5.800 & 6293296 & 784084 & 98.264 & 98.20 & $\mathrm{~N} / \mathrm{A}$ & 11546 & 3.618 & 1.23 & \\
\hline Unknown & 1 & 6.57 五 & 111197 & $\overline{14336}$ & 1.736 & 1.79 & $\overline{\mathrm{N} / \mathrm{A}}$ & 15154 & N/A & 0.97 & \\
\hline
\end{tabular}

\section{Racemic}

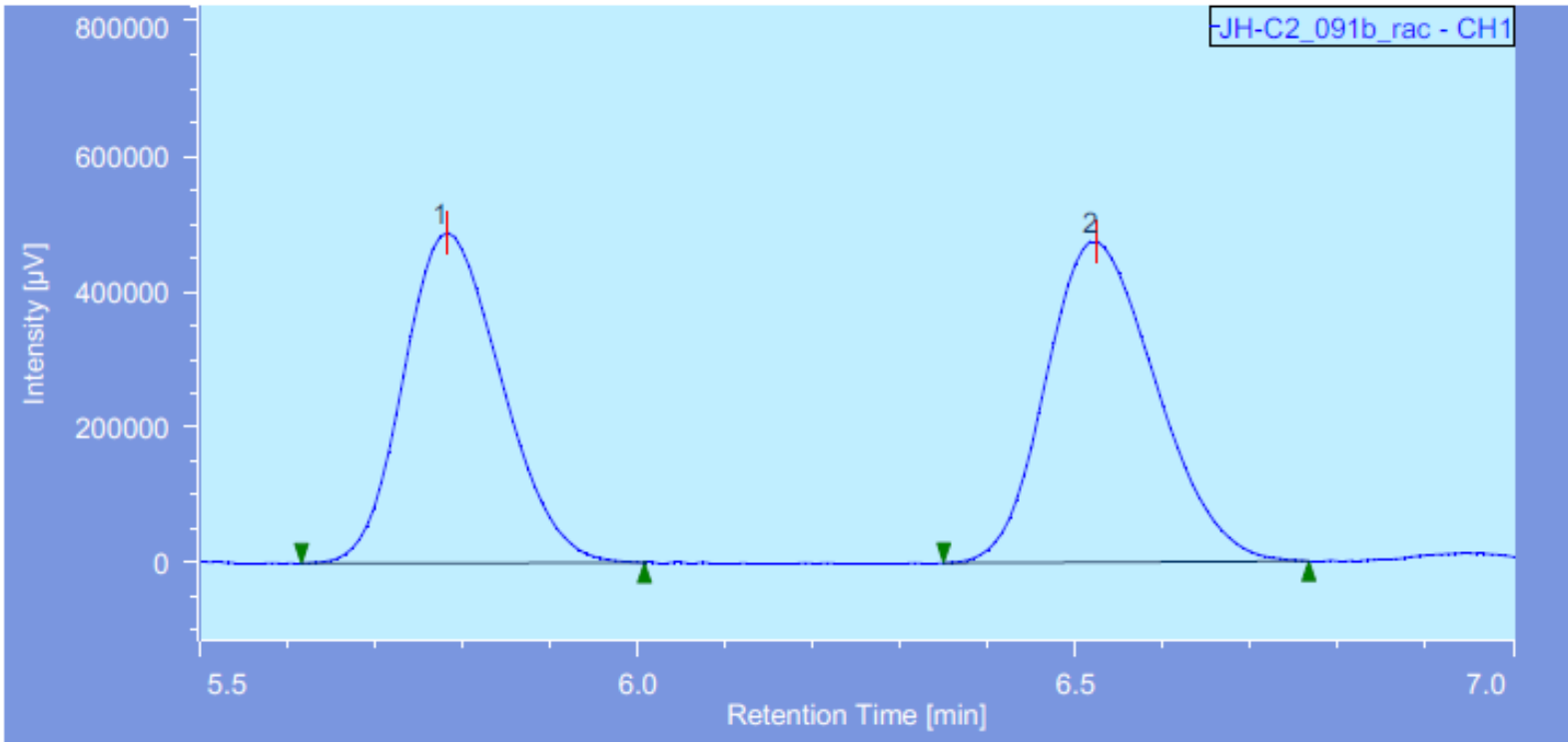

\begin{tabular}{|c|c|c|c|c|c|c|c|c|c|c|c|}
\hline Peak Name & $\mathrm{CH}$ & $t \mathbb{R}[\min ]$ & Area $[\mu \mathrm{V}-\mathrm{sec}]$ & Height $[\mu \mathrm{V}]$ & Area\% & Height\% & Quantity & NTP & Resolution & Symmetry Factor & Warning \\
\hline Unknown & 1 & 5.783 & 3781522 & 487576 & 47.720 & 50.707 & $\mathrm{~N} / \mathrm{A}$ & 12378 & 3.366 & $1.17 \mathrm{~g}$ & \\
\hline 2 Unknown & 1 & 6.525 & 4142840 & 473986 & 52.280 & 49.293 & $\mathrm{~N} / \mathrm{A}$ & 12451 & N/A & 1.165 & \\
\hline
\end{tabular}



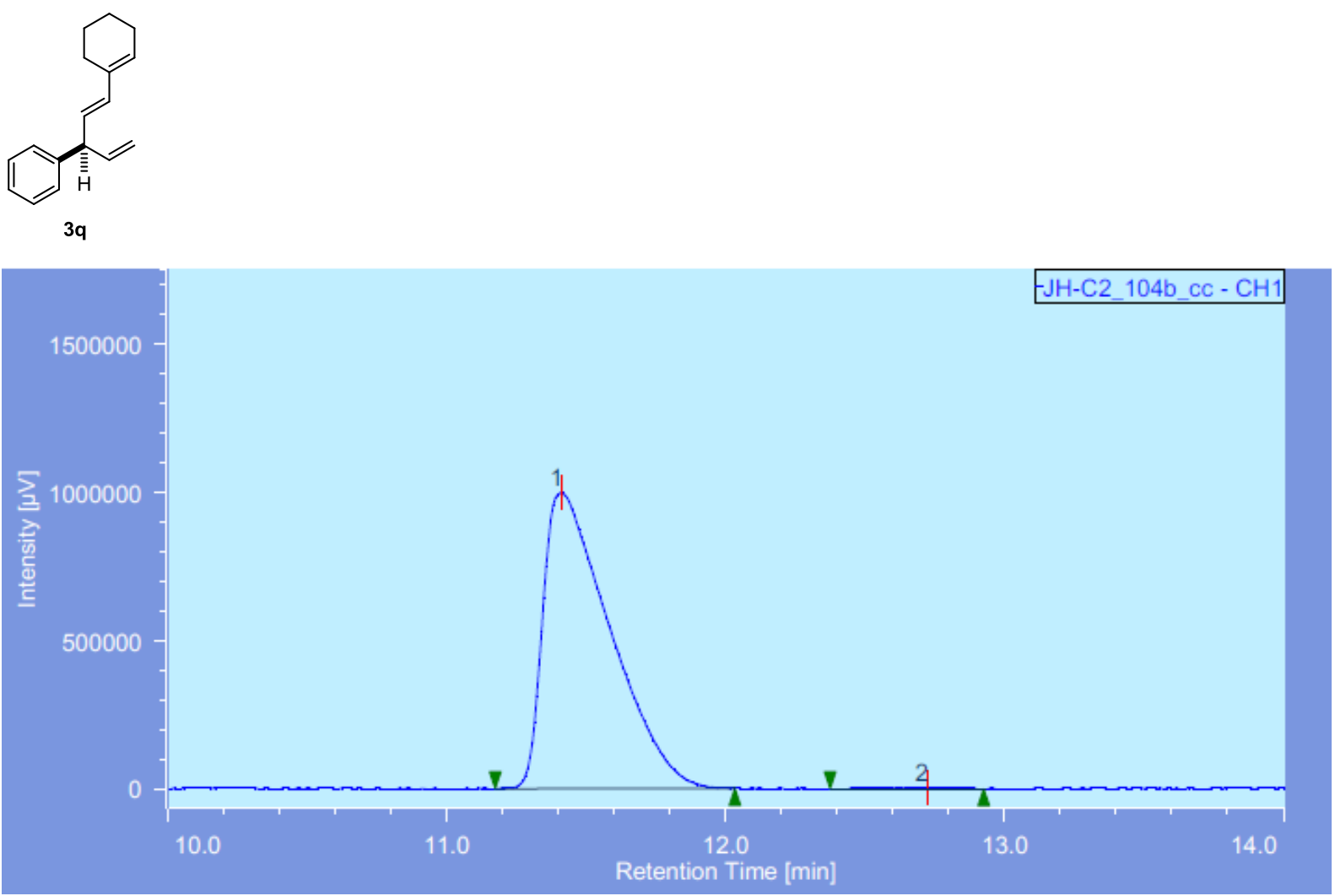

\begin{tabular}{|c|c|c|c|c|c|c|c|c|c|c|c|c|}
\hline$\#$ & Peak Name & $\mathrm{CH}$ & $\mathbb{t R}[\min ]$ & Area $[\mu \mathrm{V}$-sec] & Height $[\mu \mathrm{V}]$ & Area\% & Height $\%$ & Quantity & NTP & Resolution & Symmetry Factor & Warning \\
\hline & Unknown & 1 & 11.417 & 16605135 & 996279 & 99.697 & 99.642 & N/A & 10599 & 3.005 & 2.079 & \\
\hline & Unknown & 1 & 12.725 & 50527 & 3579 & 0.303 & 0.358 & N/A & 14053 & $\mathrm{~N} / \mathrm{A}$ & $\overline{0.846}$ & \\
\hline
\end{tabular}

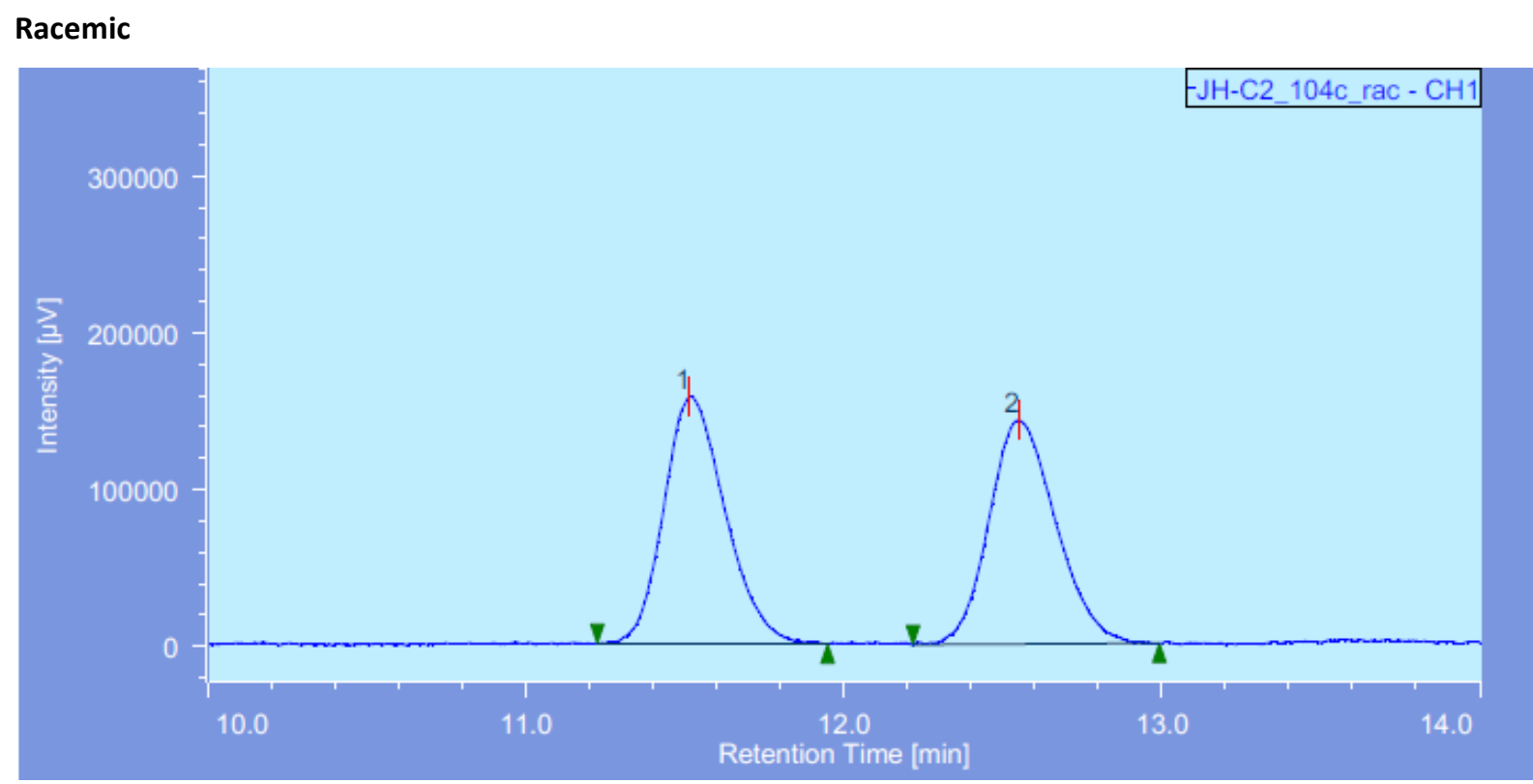

\begin{tabular}{|c|c|c|c|c|c|c|c|c|c|c|c|}
\hline Pealk & $\mathrm{CH}$ & $\operatorname{tR}[\min ]$ & Area $[\mu \mathrm{V}-\mathrm{sec}]$ & $\operatorname{cht}[\mu \mathrm{V}$ & Area\% & Heighto & intity & NTP & Resolution & Symmetry Factor & $\mathrm{Wa}$ \\
\hline & \begin{tabular}{|l|}
1 \\
\end{tabular} & 11.517 & 2115618 & 157966 & 50.397 & 52.596 & N/A & 17367 & 2.826 & 1.223 & \\
\hline Unturnown & & 1550 & 2276 & & .603 & 404 & & 122 & & & \\
\hline
\end{tabular}



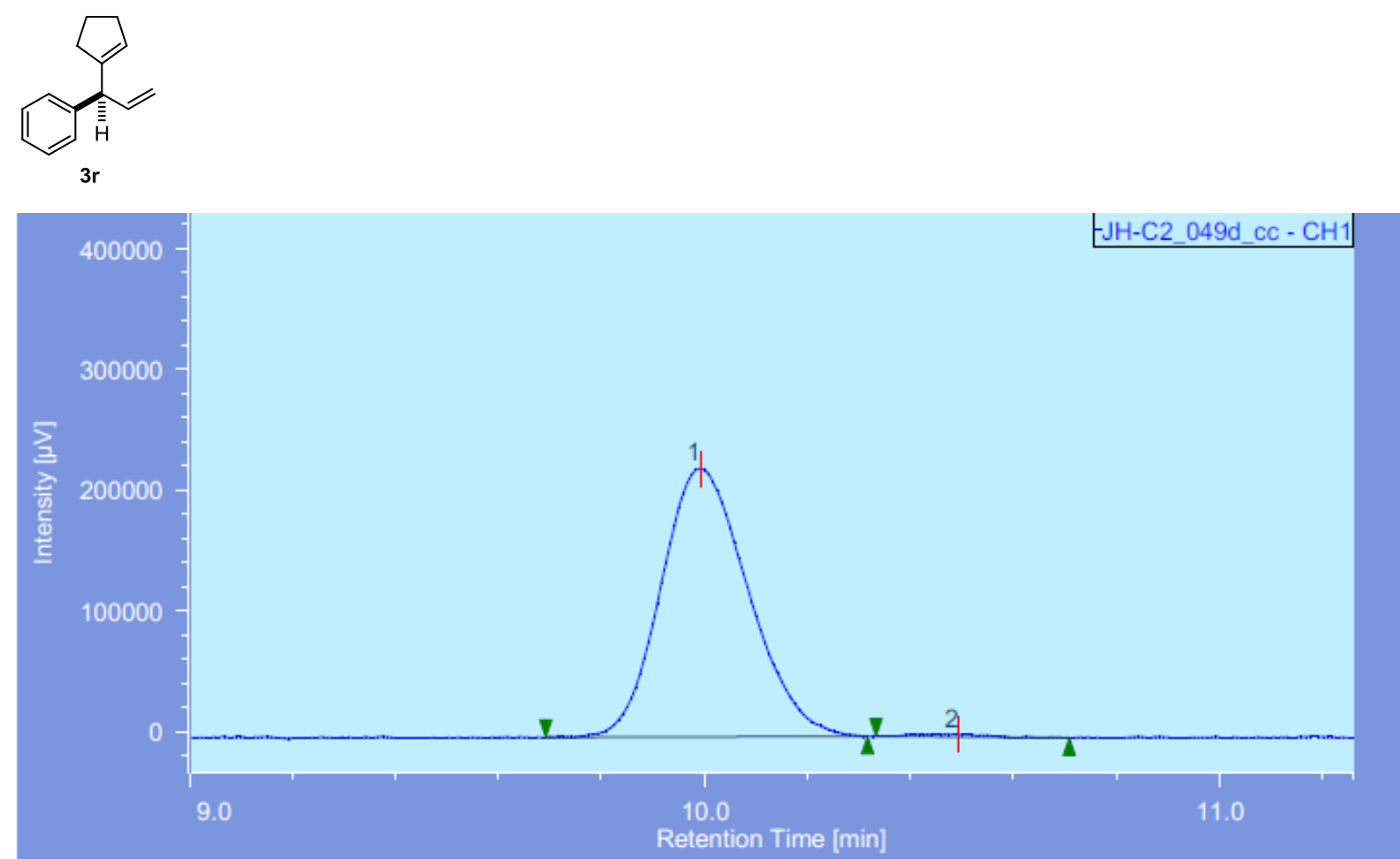

\begin{tabular}{|c|c|c|c|c|c|c|c|c|c|c|c|}
\hline \begin{tabular}{l|l}
$\#$ & Pealk Name
\end{tabular} & $\mathrm{CH}$ & $t \mathbb{R}[\min ]$ & Area $[\mu \mathrm{V}$ sec $]$ & Height $[\mu \mathrm{V}]$ & Area\% & \begin{tabular}{|l|} 
Height $\%$ \\
\end{tabular} & \begin{tabular}{|l|} 
Quantity \\
\end{tabular} & NIP & \begin{tabular}{|l|} 
Resolution \\
\end{tabular} & Symmetry Factor & \begin{tabular}{|l|l|} 
Warnings \\
\end{tabular} \\
\hline Unknown & 1 & 9.992 & 2618578 & 222324 & 99.152 & 98.934 & $\mathrm{~N} / \mathrm{A}$ & 16380 & 1.663 & 1.172 & \\
\hline Unknown & 1 & 10.492 & 22401 & 2396 & 0.848 & 1.066 & N/A & 20834 & $\mathrm{~N} / \mathrm{A}$ & 1.199 & \\
\hline
\end{tabular}

\section{Racemic}

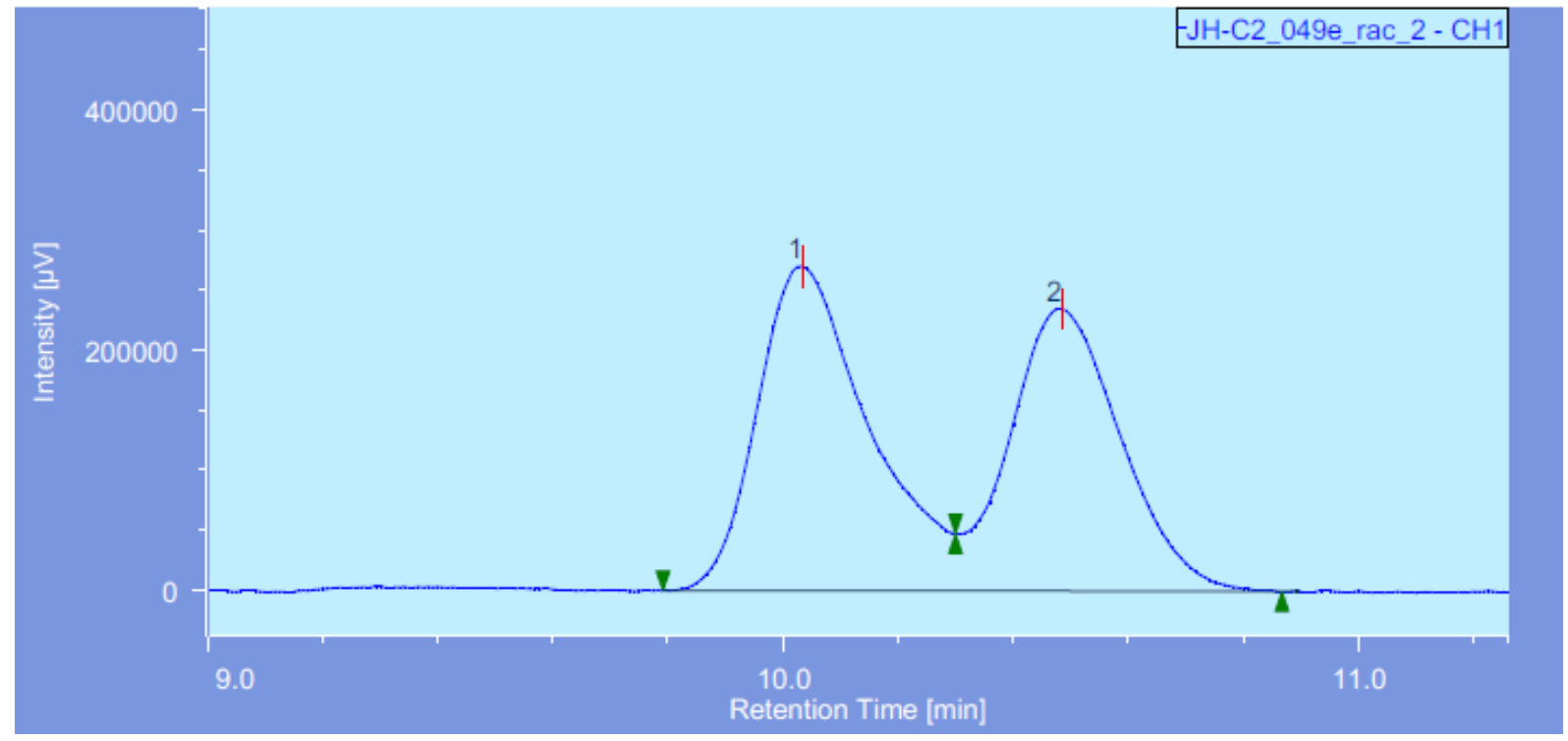

\begin{tabular}{|c|c|c|c|c|c|c|c|c|c|c|c|c|}
\hline 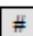 & Peak Name & $\mathrm{CH}$ & $\mathrm{tR}[\mathrm{min}]$ & Area $[\mu \mathrm{V}-\mathrm{sec}]$ & Height [ $\mu \mathrm{V}]$ & Area\% & Height $\%$ & Quantity & NIP & Resolution & Symmetry Factor & Warning \\
\hline & Unknown & 1 & 10.033 & 3653163 & 270323 & $53.56 \mathrm{~s}$ & 53.511 & $\mathrm{~N} / \mathrm{A}$ & 13747 & 1.301 & $\mathrm{~N} / \mathrm{A}$ & \\
\hline & Unknown & 1 & 10.483 & 3166504 & 234847 & 46.432 & 46.489 & $\mathrm{~N} / \mathrm{A}$ & 14238 & N/A & $\mathrm{N} / \mathrm{A}$ & \\
\hline
\end{tabular}




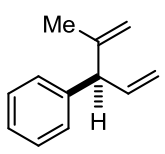

$3 s$

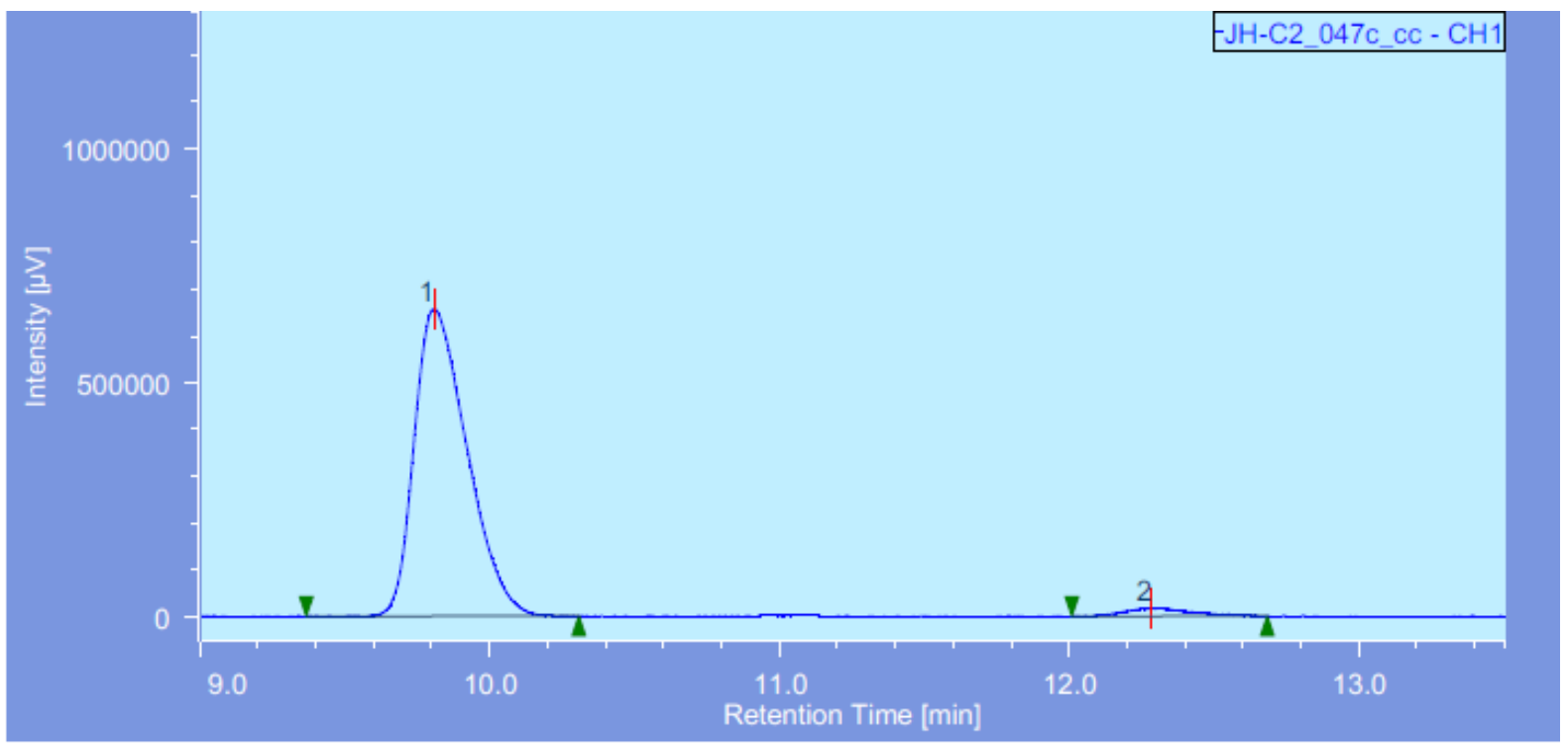

\begin{tabular}{|r|c|c|r|r|r|r|r|r|r|r|r|r|}
\hline$\#$ & Peak Name & $\mathrm{CH}$ & $\mathrm{tR}[\mathrm{min}]$ & Area $[\mu \mathrm{V}$-sec] & Height $[\mu \mathrm{V}]$ & Area\% & Height\% & Quantity & NTP & Resolution & Symmetry Factor & Warning \\
\hline 1 & Unknown & 1 & 9.808 & 8315361 & 656673 & 96.917 & 97.523 & N/A & 13633 & 6.642 & \\
\hline 2 & Unknown & 1 & 12.283 & 264486 & 16681 & 3.083 & 2.477 & N/A & 14278 & N/A & 1.444 & \\
\hline
\end{tabular}

\section{Racemic}

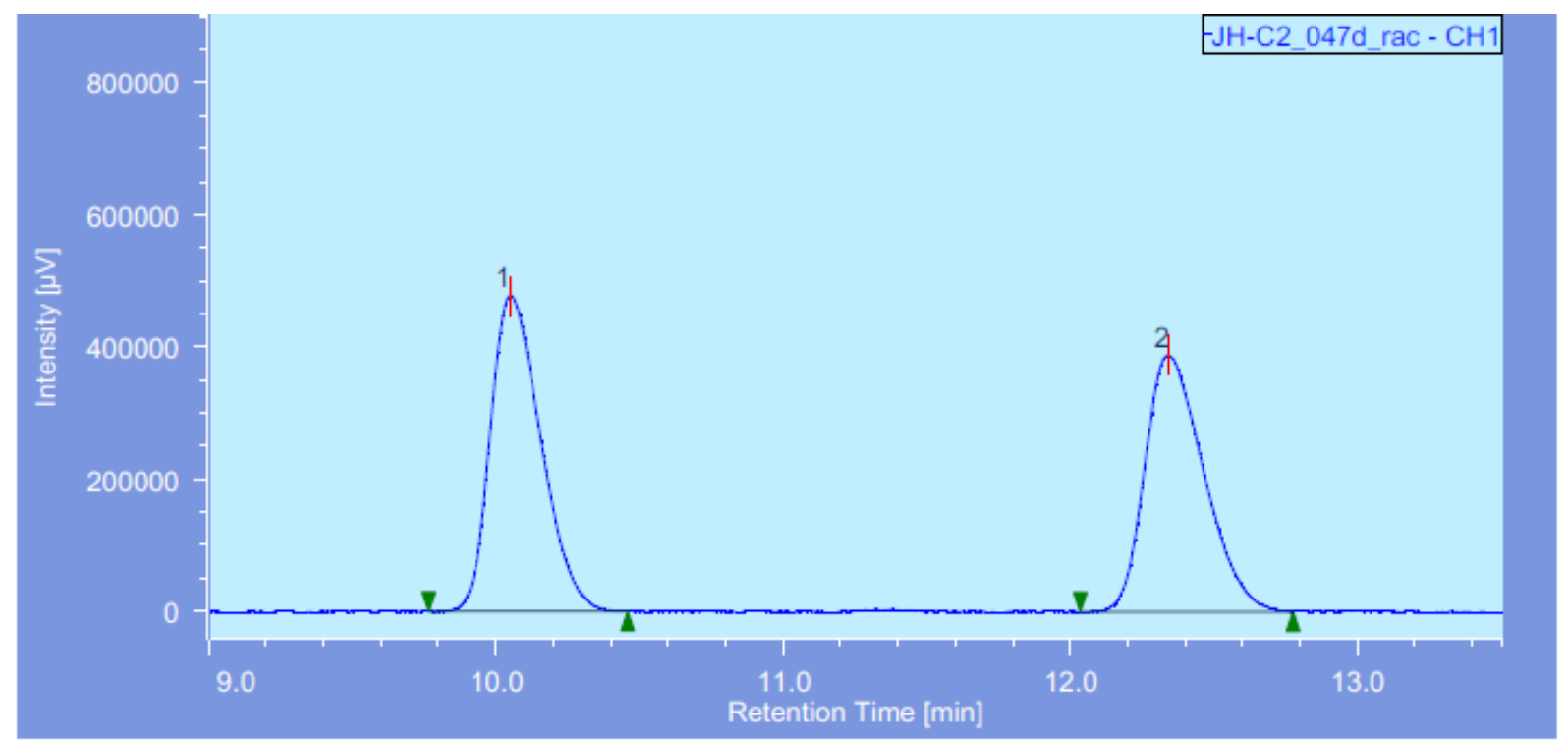

\begin{tabular}{|c|c|c|c|c|c|c|c|c|c|c|c|c|}
\hline$\#$ & Peak Name & $\mathrm{CH}$ & $t \mathbb{R}[\min ]$ & Area $[\mu \mathrm{V}-\mathrm{sec}]$ & Height $[\mu \mathrm{V}]$ & Area\% & Height $\%$ & Quantity & NTP & Resolution & Symmetry Factor & Warning \\
\hline & Unknown & 1 & 10.050 & 5819969 & 477521 & 51.308 & 55.171 & $\mathrm{~N} / \mathrm{A}$ & 15383 & 6.551 & 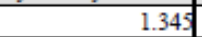 & \\
\hline & Unknown & $\overline{1}$ & 12.342 & 5523300 & 388000 & 48.692 & 44.829 & $\mathrm{~N} / \mathrm{A}$ & 17108 & N/A & 1.344 & \\
\hline
\end{tabular}




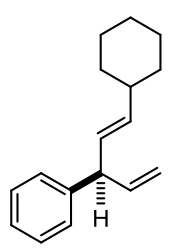

$3 t$

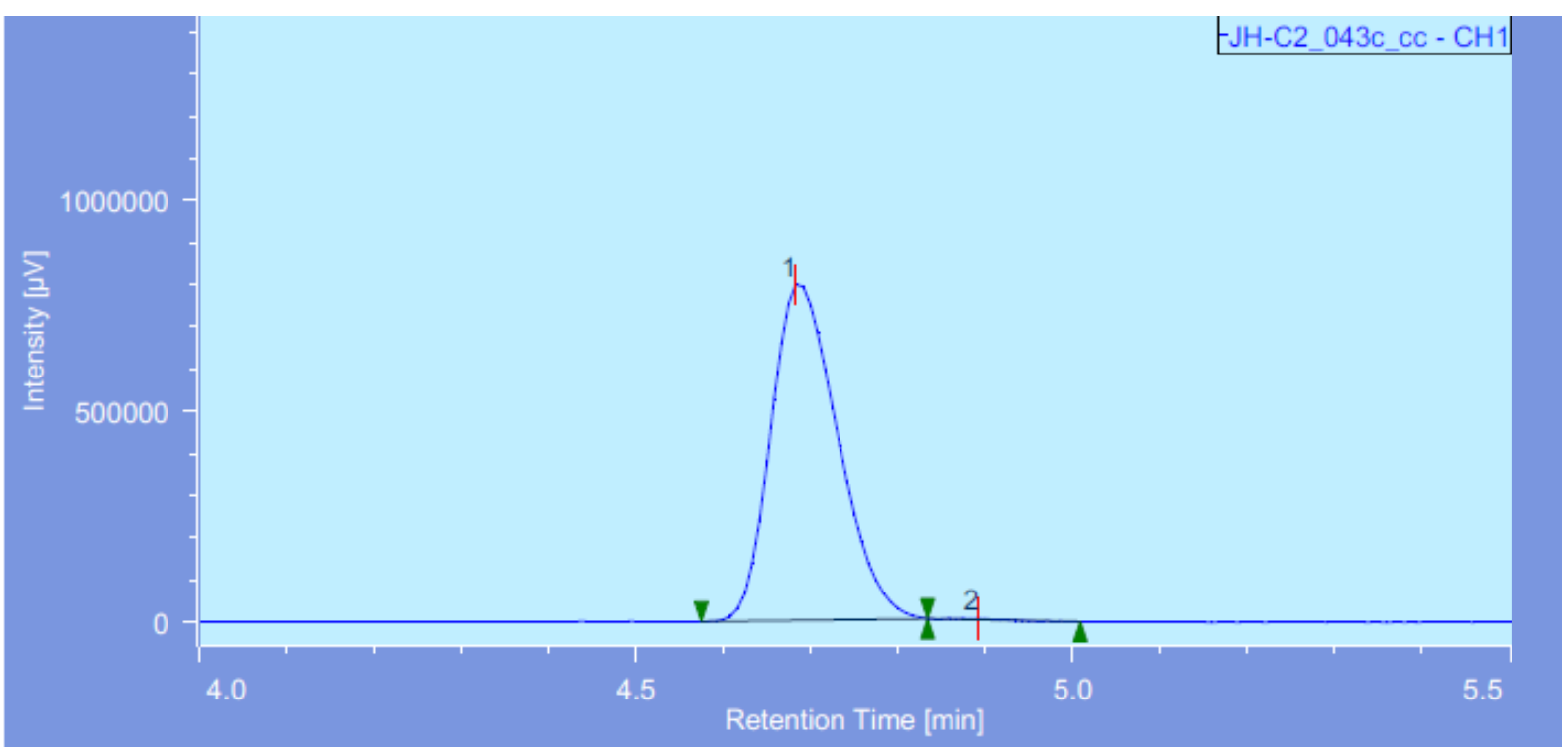

\begin{tabular}{|c|c|c|c|c|c|c|c|c|c|c|c|}
\hline Peak Name & $\mathrm{CH}$ & $t R$ [min] & Area $[\mu \mathrm{V}$-sec $]$ & Height $[\mu \mathrm{V}]$ & Area\% & Height\% & Quantity & NTP & Resolution & Symmetry Factor & Warning \\
\hline Unknown & 1 & 4.68 & 4247231 & 796697 & 99.848 & 99.685 & $\mathrm{~N} / \mathrm{A}$ & 17272 & 1.958 & 1.338 & \\
\hline 2 Unknown & $\overline{1}$ & 4.89 & 6486 & 2520 & 0.152 & 0.315 & $\mathrm{~N} / \mathrm{A}$ & 76250 & $\mathrm{~N} / \mathrm{A}$ & 1.776 & \\
\hline
\end{tabular}

\section{Racemic}

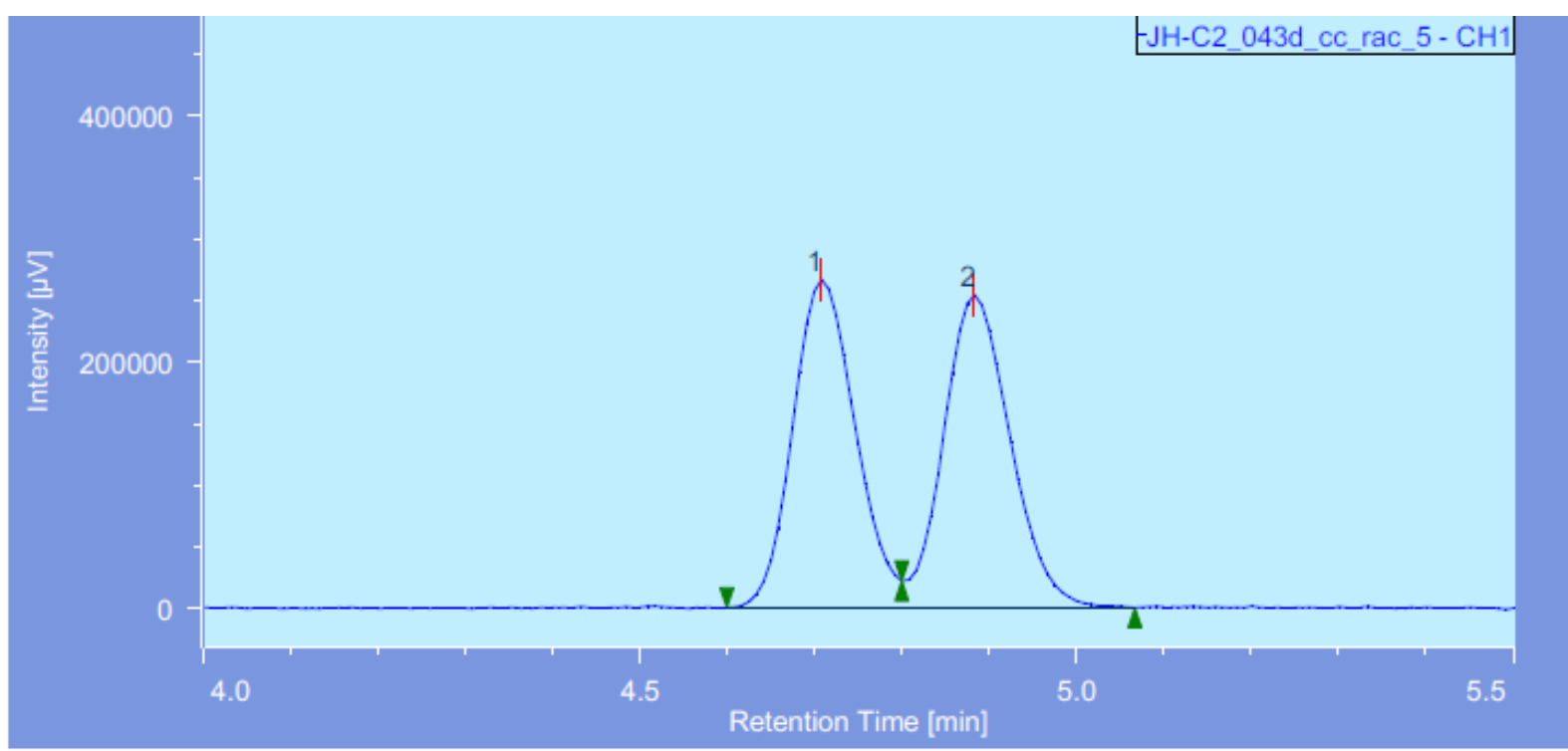

\begin{tabular}{|c|c|c|c|c|c|c|c|c|c|c|c|c|}
\hline$\#$ & Peak Name & $\mathrm{CH}$ & $\mathrm{tR}$ [min] & Area [ $\mu \mathrm{V}$-sec] & Height $[\mu \mathrm{V}]$ & Area\% & Height $\%$ & Quantity & NTP & Resolution & Symmetry Factor & Warning \\
\hline & Unknown & 1 & 4.708 & 1316438 & 265803 & 49.485 & 51.219 & $\mathrm{~N} / \mathrm{A}$ & 20424 & 1.296 & $\mathrm{~N} / \mathrm{A}$ & \\
\hline & Unknown & 1 & 4.883 & 1343825 & 253148 & 50.515 & 48.781 & $\mathrm{~N} / \mathrm{A}$ & 19755 & $\mathrm{~N} / \mathrm{A}$ & $\mathrm{N} / \mathrm{t}$ & \\
\hline
\end{tabular}



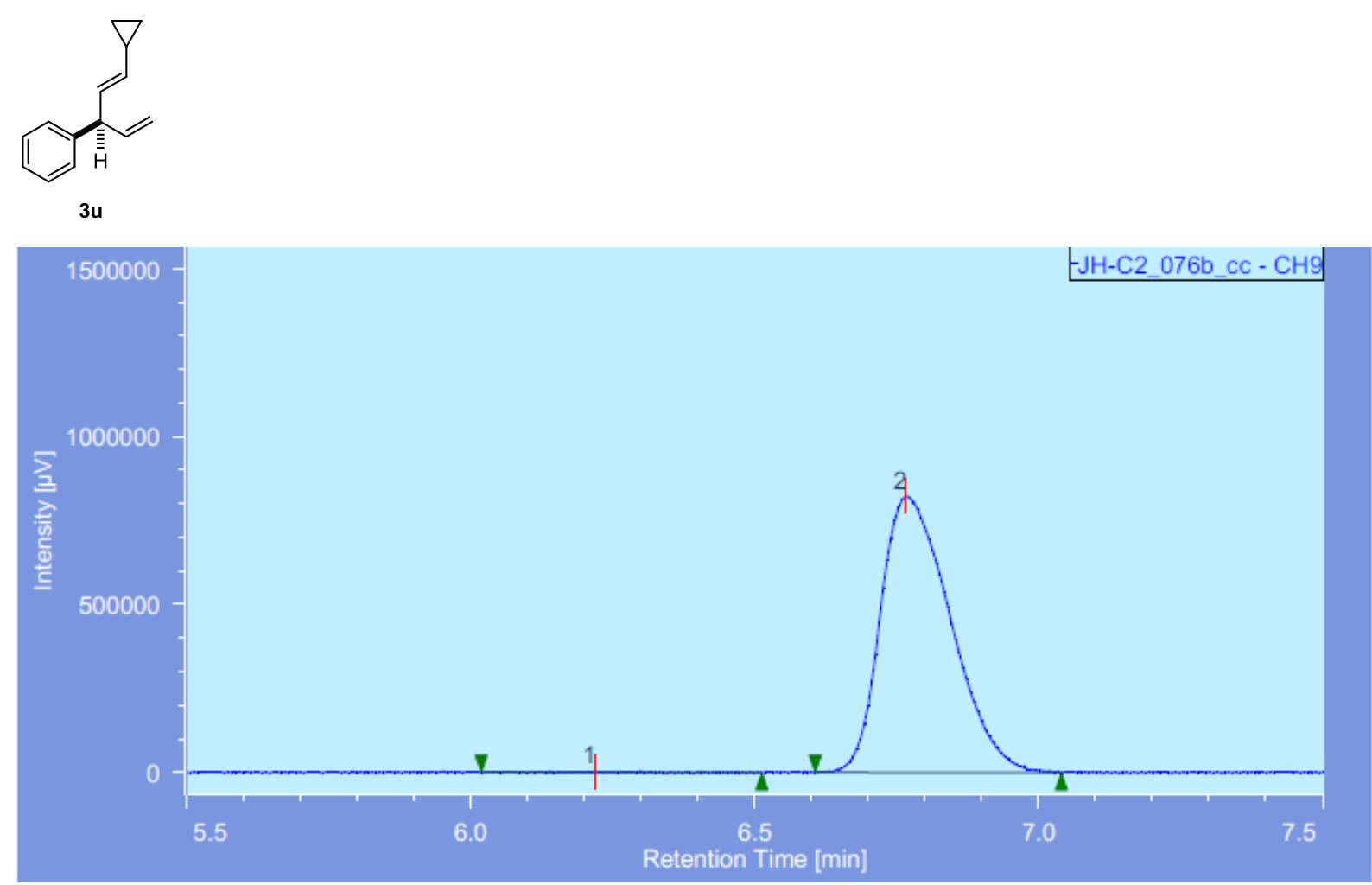

\begin{tabular}{|c|c|c|r|r|r|r|r|r|r|r|r|r}
\hline$\#$ & Peak Name & $\mathrm{CH}$ & $\mathrm{tR}[\mathrm{min}]$ & Area $[\mu \mathrm{V}$-sec] & Height $[\mu \mathrm{V}]$ & Area\% & Height\% & Quantity & NTP & Resolution & Symmetry Factor & Warning \\
\hline 2 & Unknown & 9 & 6.22 & 40430 & 3563 & 0.573 & 0.431 & N/A & 997 & 1.079 & 1.23 & \\
\hline 1 Unknown & 9 & 6.76 & 7011295 & 822236 & 99.427 & 99.569 & N/A & 14072 & N/A & & 1.440 & \\
\hline
\end{tabular}

\section{Racemic}

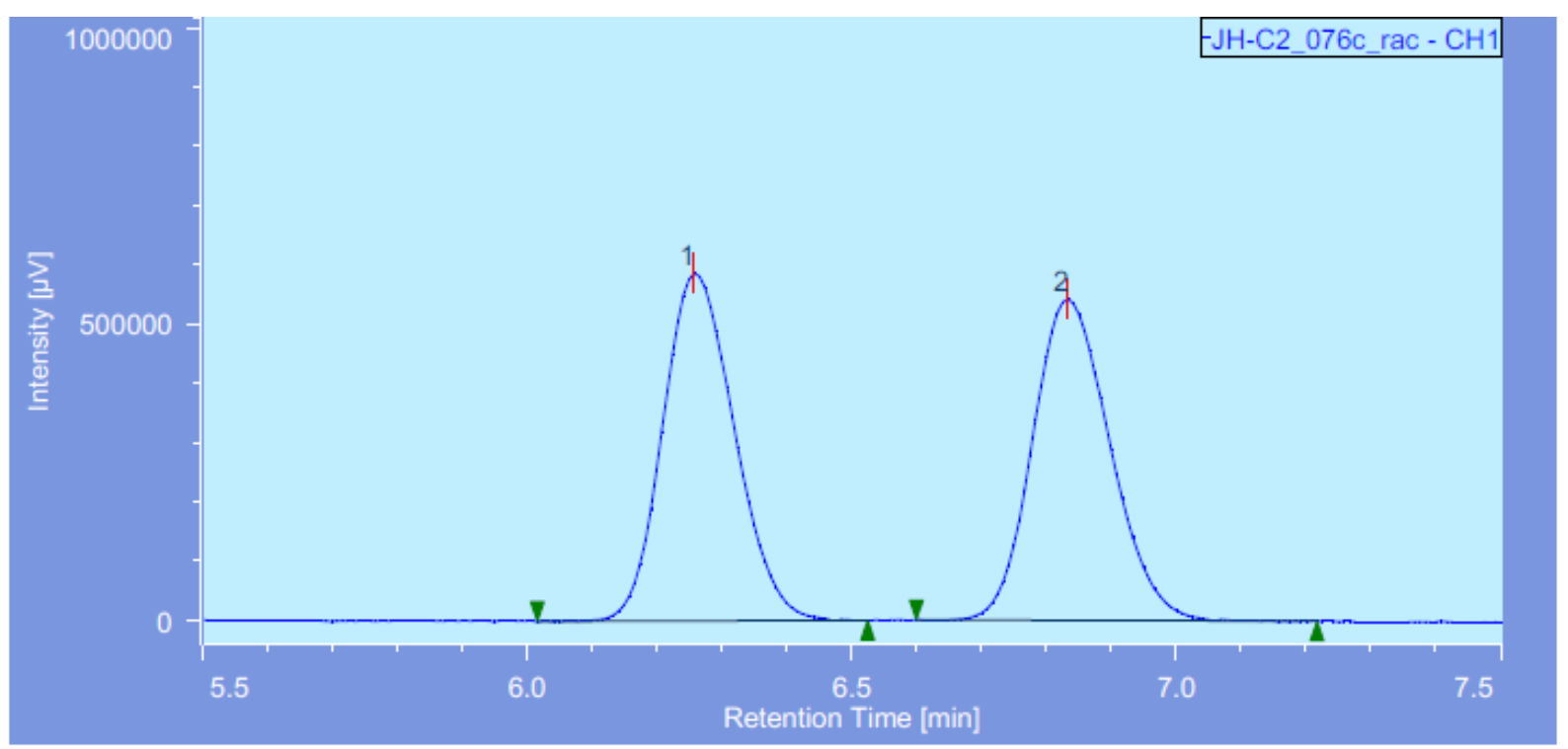

\begin{tabular}{|c|c|c|c|c|c|c|c|c|c|c|c|c|}
\hline$\#$ & Peak Name & $\mathrm{CH}$ & $\mathrm{tR}[\mathrm{min}]$ & Area [ $\mu \mathrm{V}$-sec] & Height $[\mu \mathrm{V}]$ & Area\% & Height $\%$ & Quantity & NTP & Resolution & Symmetry Factor & Warning \\
\hline & Unknown & 1 & 6.258 & 4485233 & 586315 & 49.939 & 51.976 & N/A & 15119 & 2.718 & 1.168 & \\
\hline & Unknown & 1 & 6.833 & 4496111 & 541734 & 50.061 & 48.024 & N/A & 15346 & N/A & 1.154 & \\
\hline
\end{tabular}


<smiles>C=C[C@H](/C=C\c1ccc(OC)cc1)c1ccc(OC)cc1</smiles>

s1

\section{$0.25 \mathrm{mmol}$ scale}

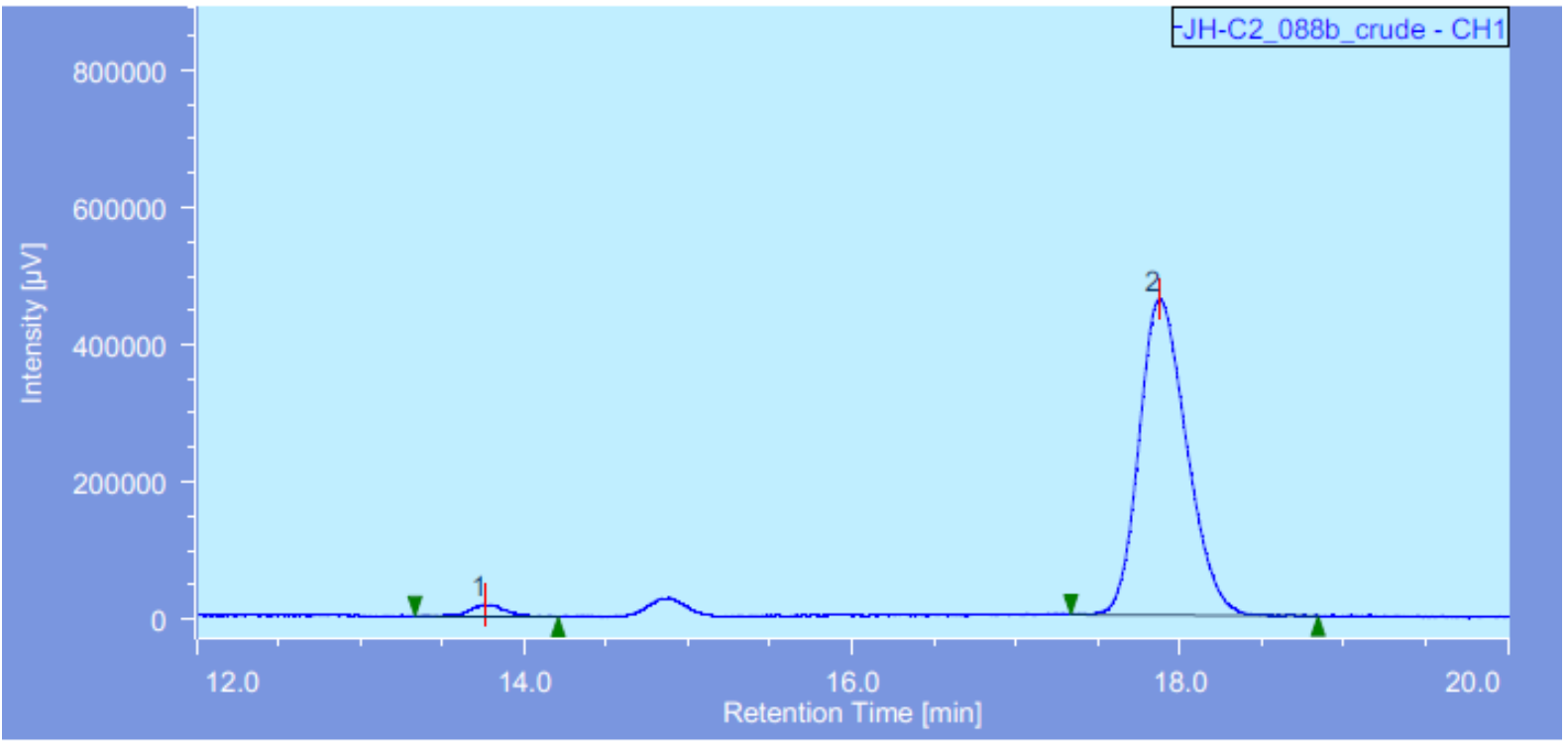

\begin{tabular}{|c|c|c|c|c|c|c|c|c|c|c|c|c|}
\hline$\#$ & Peak Name & $\mathrm{CH}$ & tR [min] & Area [ $[\mu \mathrm{V}$-sec] & Height $[\mu \mathrm{V}]$ & Area $\%$ & Height $\%$ & Quantity & NIP & Resolution & Symmetry Factor & Warning \\
\hline & Unknown & 1 & 13.767 & 267009 & 17253 & 2.801 & 3.608 & $\mathrm{~N} / \mathrm{A}$ & 19396 & 8.909 & 1.252 & \\
\hline 2 & Unknown & 1 & 17.875 & 9266583 & 460903 & 97.199 & 96.392 & $\mathrm{~N} / \mathrm{A}$ & 18242 & $\mathrm{~N} / \mathrm{A}$ & 1.221 & \\
\hline
\end{tabular}

\section{$7.0 \mathrm{mmol} \mathrm{scale}$}

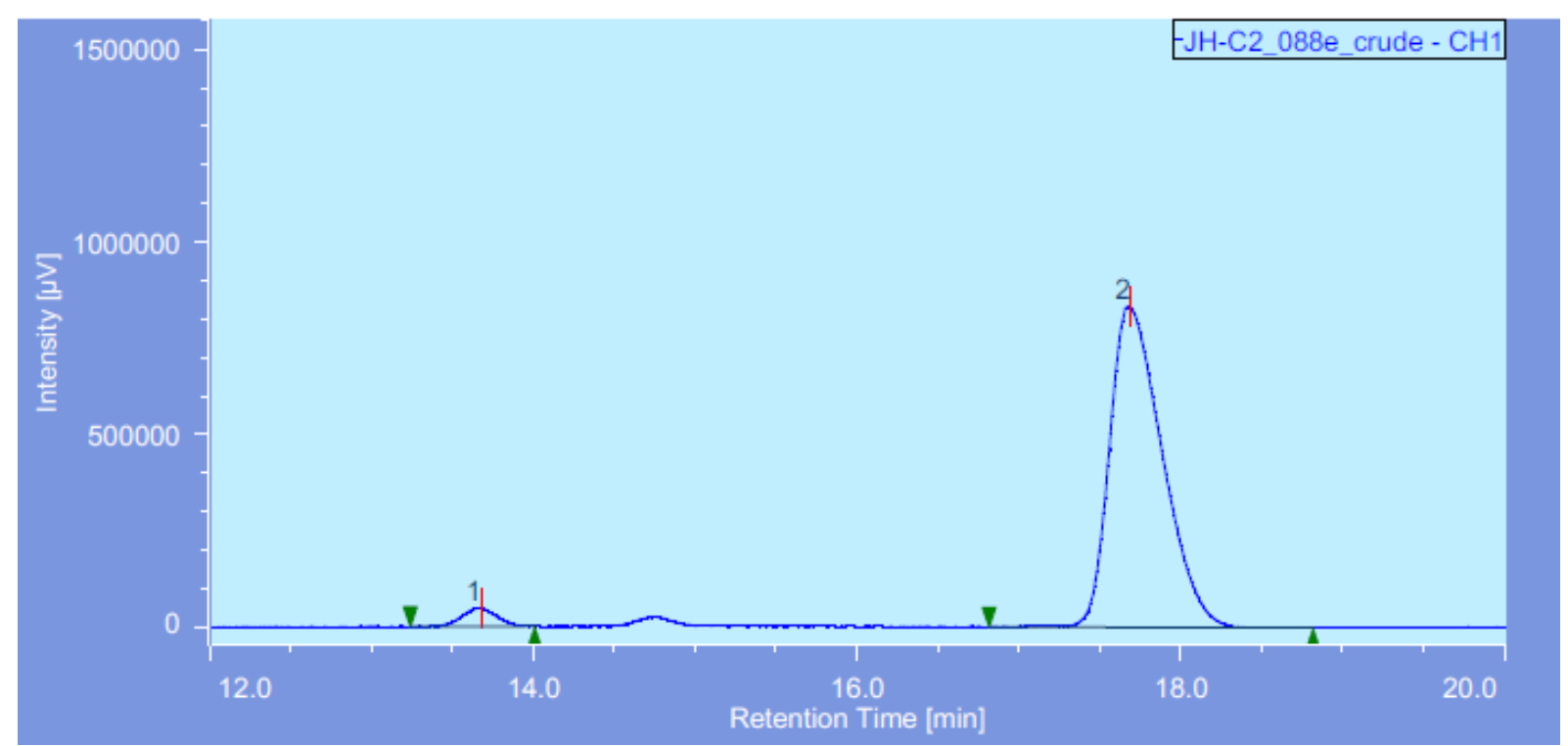

\begin{tabular}{|c|c|c|c|c|c|c|c|c|c|c|c|c|}
\hline \# & Pealk Name & $\mathrm{CH}$ & $t \mathbb{R}[\min ]$ & Area $[\mu \mathrm{V}$-sec] & Height $[\mu \mathrm{V}]$ & Area\% & Height $\%$ & Quantity & NTP & Resolution & Symmetry Factor & Warning \\
\hline & Unknown & 1 & 13.675 & 706346 & 47885 & 3.620 & 5.437 & $\mathrm{~N} / \mathrm{A}$ & 19795 & 8.161 & 0.974 & \\
\hline & Unknown & 1 & 17.683 & 18804206 & 832814 & 96.380 & 94.563 & $\mathrm{~N} / \mathrm{A}$ & 14077 & $\mathrm{~N} / \mathrm{A}$ & 1.421 & \\
\hline
\end{tabular}




\section{Racemic}

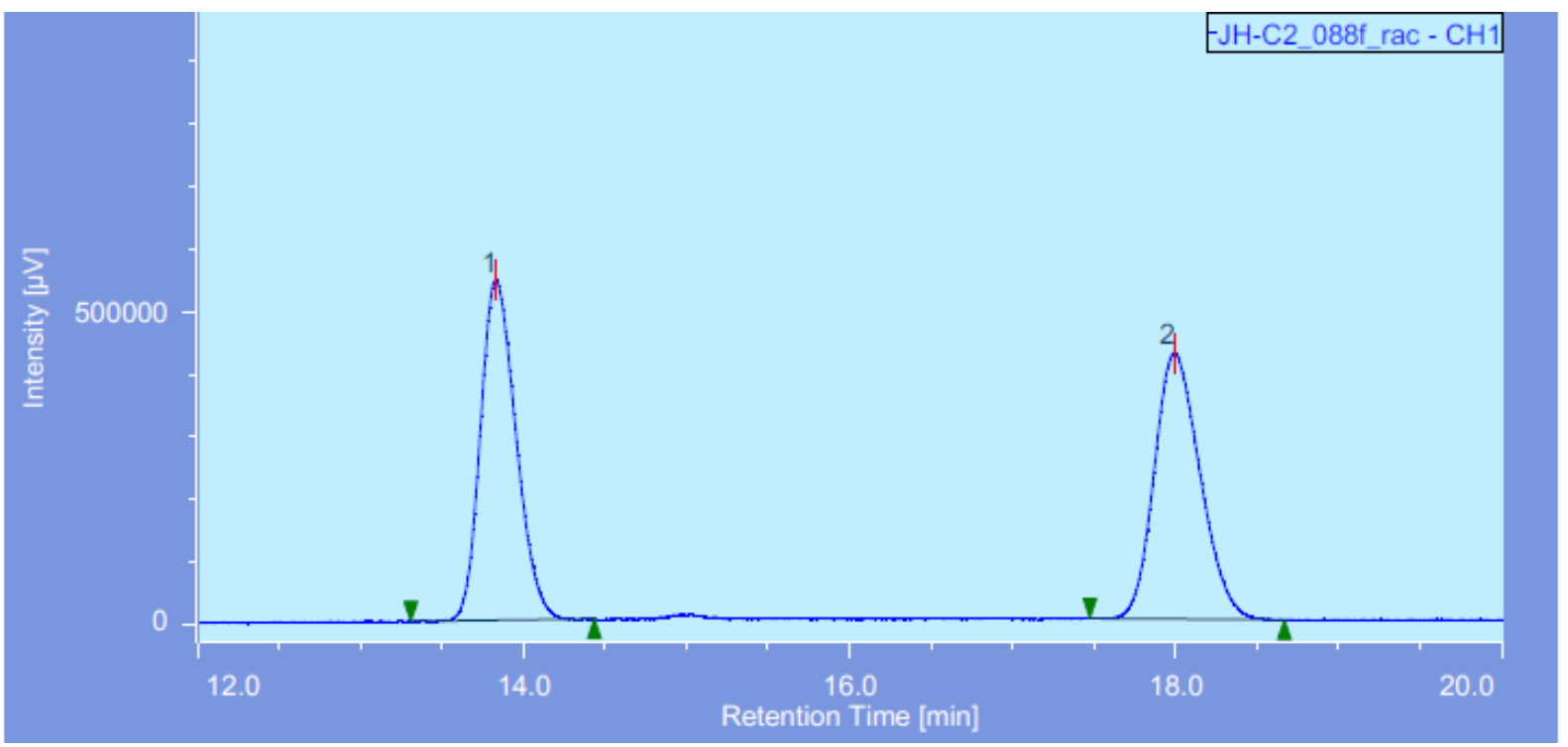

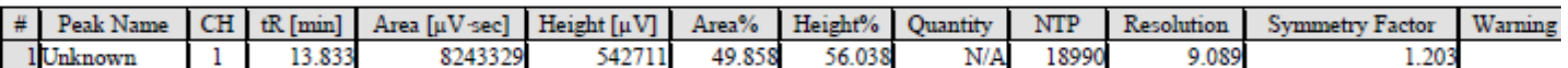

\begin{tabular}{|c|c|c|c|c|c|c|c|c|c|c|c|}
\hline Unknown & 1 & 13.833 & 8243329 & 542711 & 49.858 & 56.038 & N/A & 18990 & 9.089 & 1.203 & \\
\hline Unknown & l & 17.992 & 8290220 & 425758 & 50.142 & 43.962 & N/A & 19457 & N/A & 1.19 & \\
\hline
\end{tabular}



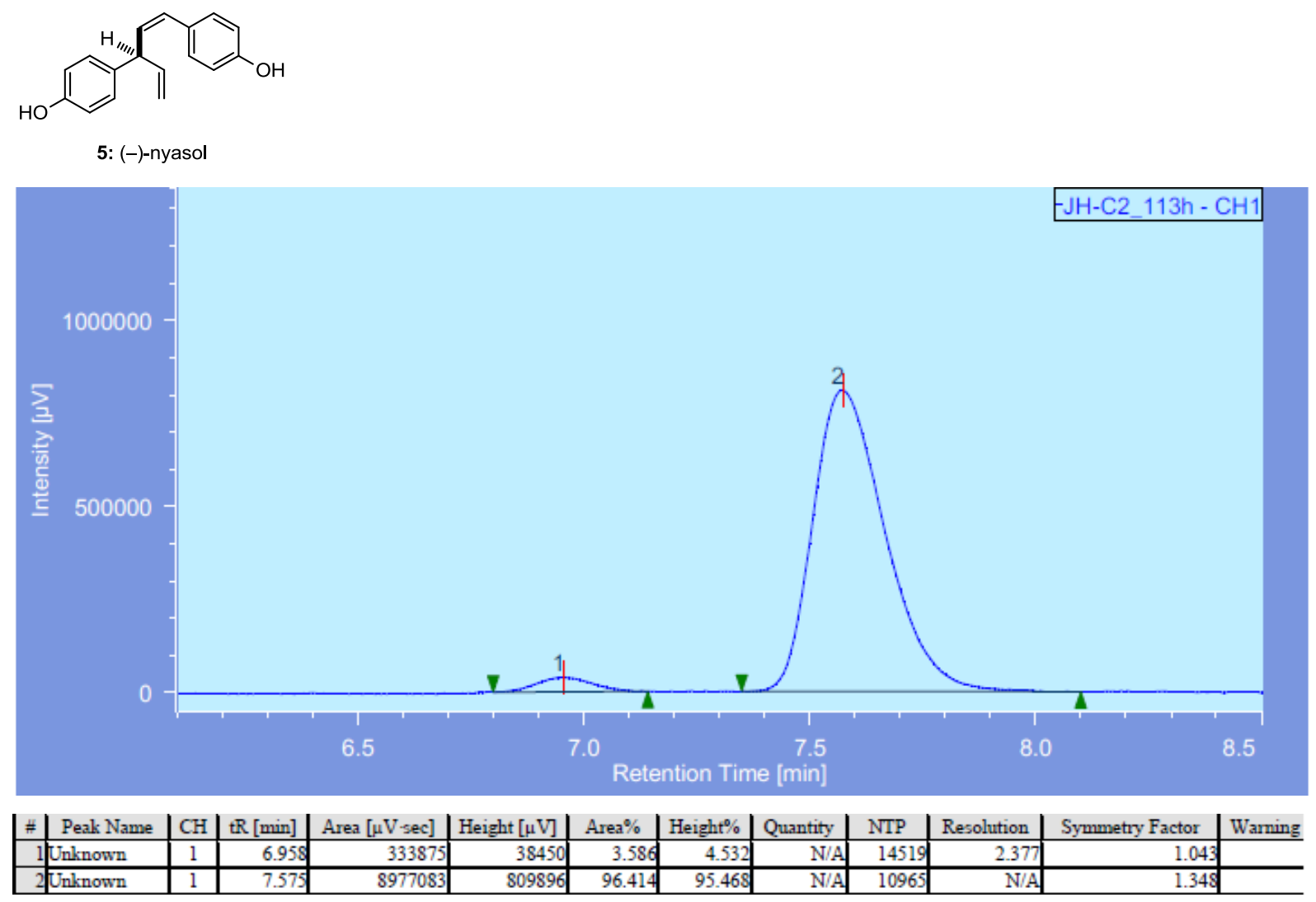

\section{Racemic}

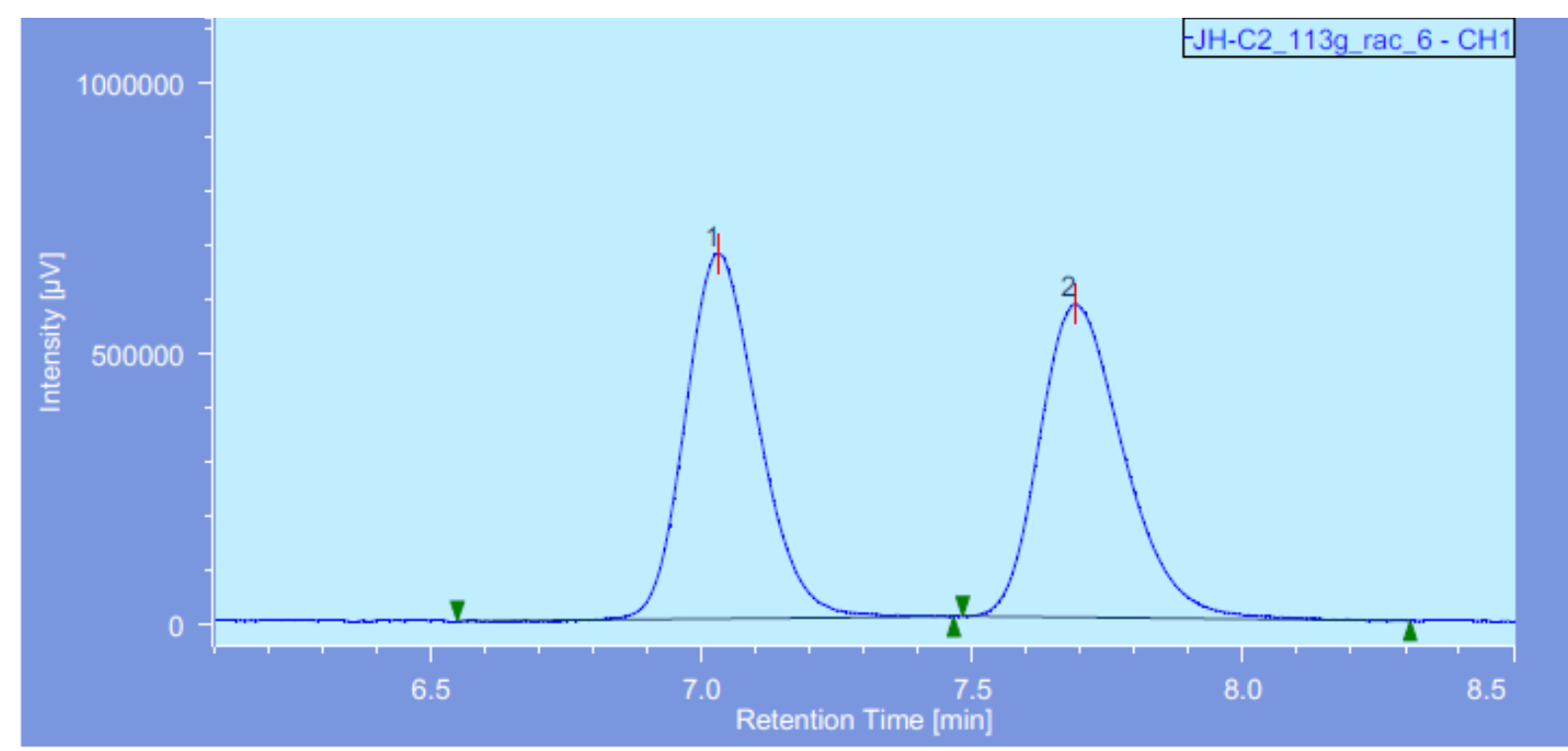

\begin{tabular}{|c|c|c|c|c|c|c|c|c|c|c|c|}
\hline \begin{tabular}{|l|l|l|}
$\#$ & Peak Name \\
\end{tabular} & $\mathrm{CH}$ & $t \mathbb{R}[\min ]$ & Area $[\mu \mathrm{V}-\mathrm{sec}]$ & Height $[\mu \mathrm{V}]$ & Area\% & Height\% & Quantity & NTP & Resolution & Symmetry Factor & Warning \\
\hline Unknown & 1 & 7.03 & 6317022 & 674442 & 50.21 & 53.80 & N/A & 13271 & 2.512 & 1.17 & \\
\hline Unknown & 1 & 7.69 & 6263692 & 579146 & 49.78 & 46.19 & N/A & 11960 & N/A & 1.29 & \\
\hline
\end{tabular}



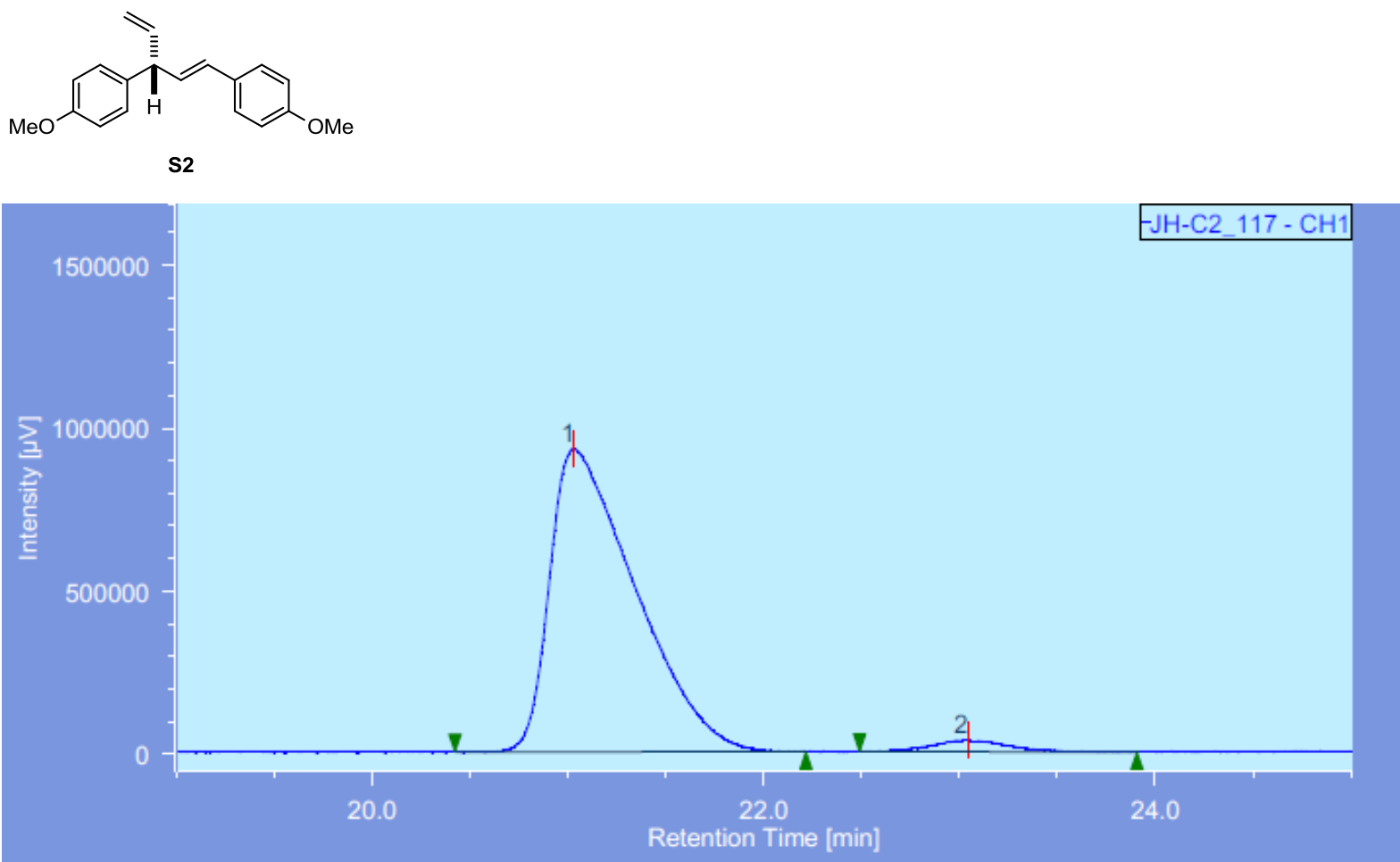

\begin{tabular}{|c|c|c|c|c|c|c|c|c|c|c|c|}
\hline \begin{tabular}{|l|l|}
$\#$ & Peak Name \\
\end{tabular} & $\mathrm{CH}$ & $t \mathbb{R}[\min ]$ & Area $[\mu \mathrm{V} \cdot \mathrm{sec}]$ & Height $[\mu \mathrm{V}]$ & Area\% & \begin{tabular}{|l|} 
Height $\%$ \\
\end{tabular} & \begin{tabular}{|l|} 
Quantity \\
\end{tabular} & NTP & Resolution & Symmetry Factor & Warning \\
\hline Unknown & 1 & 21.033 & 28181664 & 929716 & 96.760 & 96.439 & $\mathrm{~N} / \mathrm{A}$ & 10906 & 2.636 & 1.944 & \\
\hline Unknown & 1 & 23.042 & 943743 & 34333 & 3.240 & 3.561 & N/A & 1628 & N/A & 1.091 & \\
\hline
\end{tabular}

\section{Racemic}

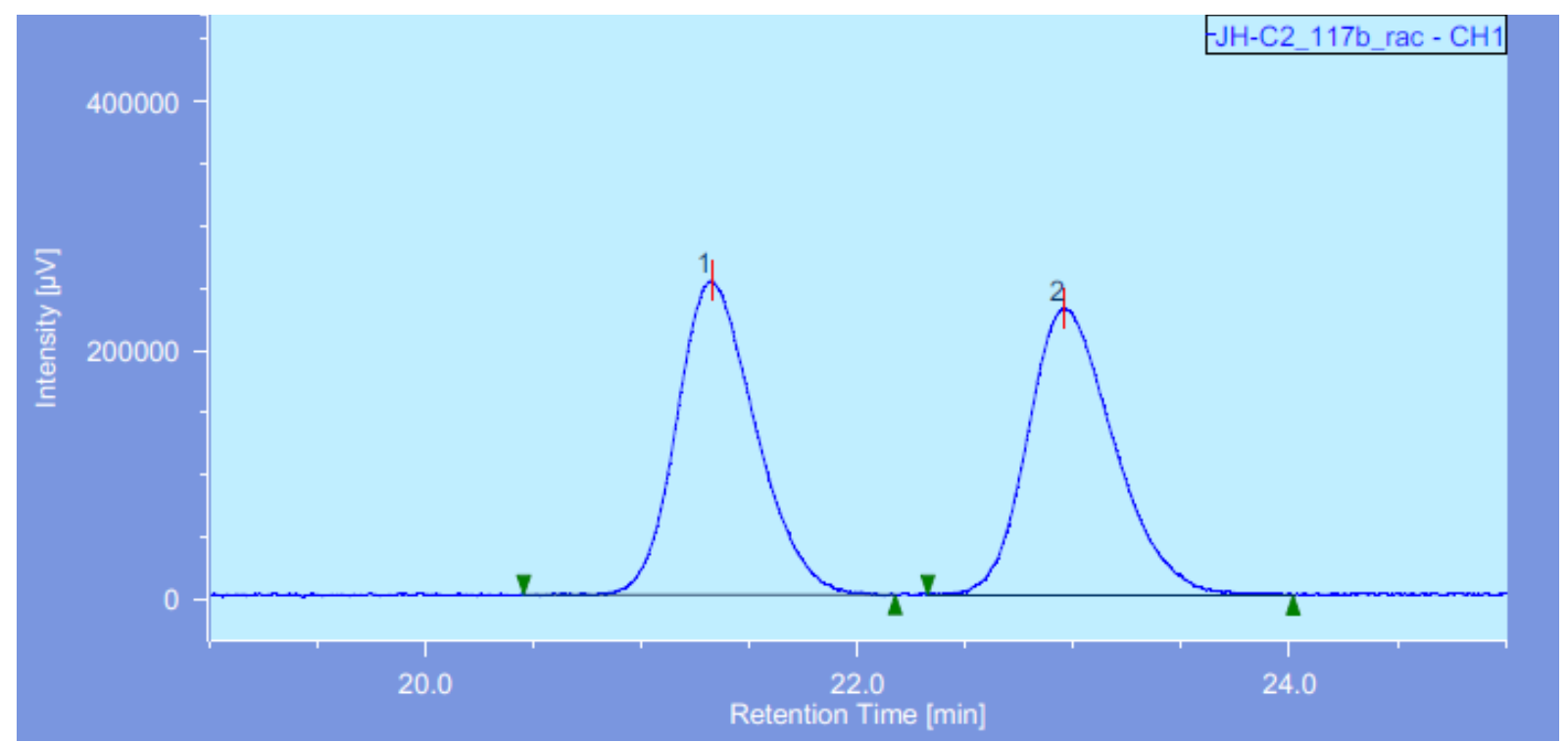

\begin{tabular}{|c|c|c|c|c|c|c|c|c|c|c|c|}
\hline \begin{tabular}{l|l}
$\#$ & Peak Name
\end{tabular} & $\mathrm{CH}$ & $t \mathbb{R}[\min ]$ & Area $[\mu \mathrm{V}$-sec $]$ & Height $[\mu \mathrm{V}]$ & Area\% & Height\% & Quantity & NTP & \begin{tabular}{|l|l|l|l} 
Resolution \\
\end{tabular} & Symmetry Factor & Warning \\
\hline Unknown & 1 & 21.32 & 6377980 & 251879 & 49.80 & 52.21 & N/A & 16459 & 2.356 & 1.202 & \\
\hline Unknown & 1 & 22.95 & 6428862 & 230491 & 50.19 & 47.78 & N/A & 16019 & N/A & 1.253 & \\
\hline
\end{tabular}


<smiles>C=C[C@@H](C=Cc1ccc(O)cc1)c1ccc(O)cc1</smiles>

6: (-)-hinokiresinol

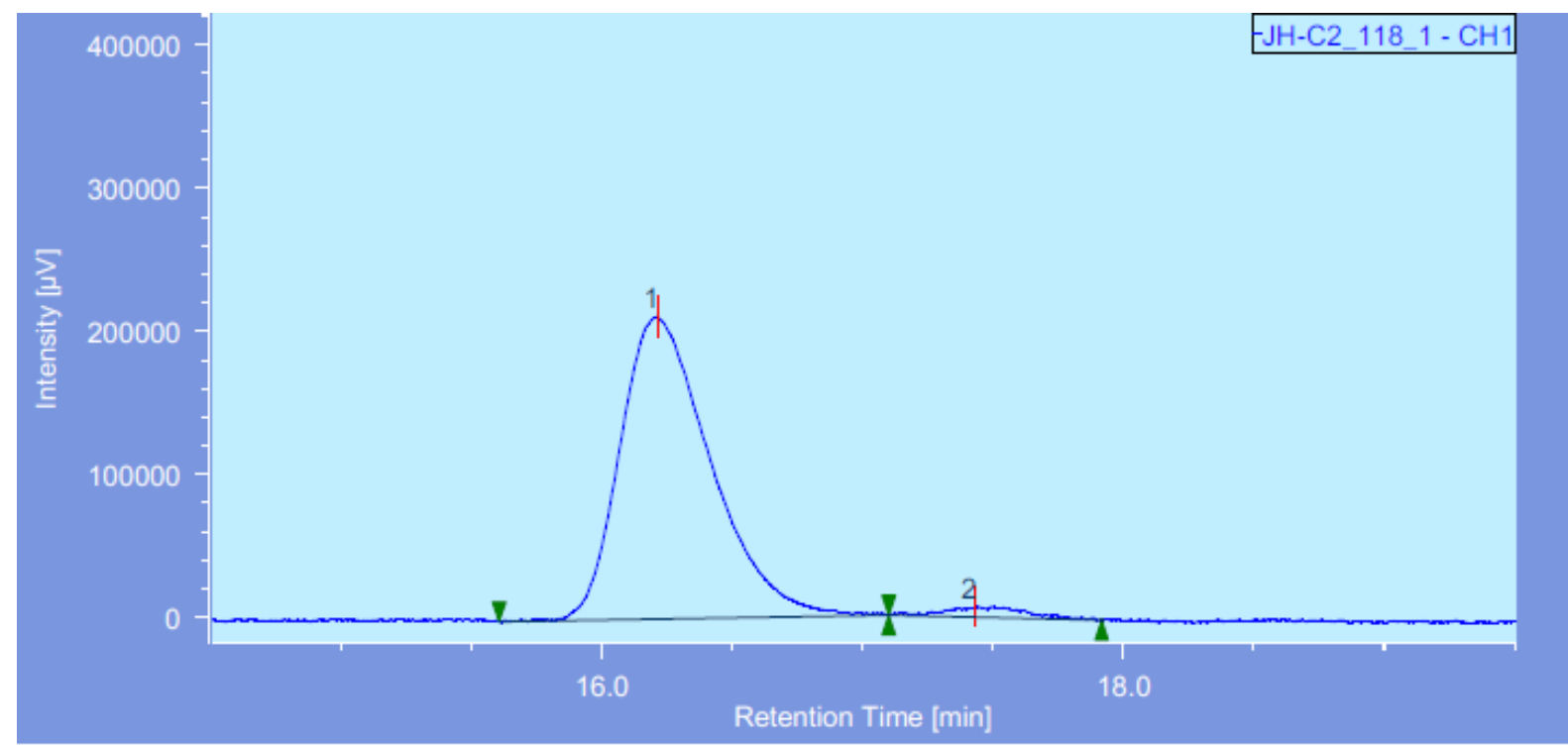

\begin{tabular}{|c|c|c|c|c|c|c|c|c|c|c|c|}
\hline \begin{tabular}{l|l}
$\#$ Pealk Name \\
\end{tabular} & $\mathrm{CH}$ & $t \mathbb{R}[\min ]$ & Area $[\mu \mathrm{V} \cdot \mathrm{sec}]$ & Height $[\mu \mathrm{V}]$ & Area\% & Height $\%$ & \begin{tabular}{|l|} 
Quantity \\
\end{tabular} & NIP & \begin{tabular}{|l} 
Resolution \\
\end{tabular} & Symmetry Factor & \begin{tabular}{|l|} 
Warning \\
\end{tabular} \\
\hline Unknown & 1 & 16.21 & 5103137 & 211399 & $96.85 !$ & 96.713 & $\mathrm{~N} / \mathrm{A}$ & 10696 & 2.038 & 1.386 & \\
\hline Unknown & 1 & 17.43 & 165500 & 7184 & 3.14 & 3.287 & $\mathrm{~N} / \mathrm{A}$ & 14985 & N/A & 1.203 & \\
\hline
\end{tabular}

\section{Racemic}

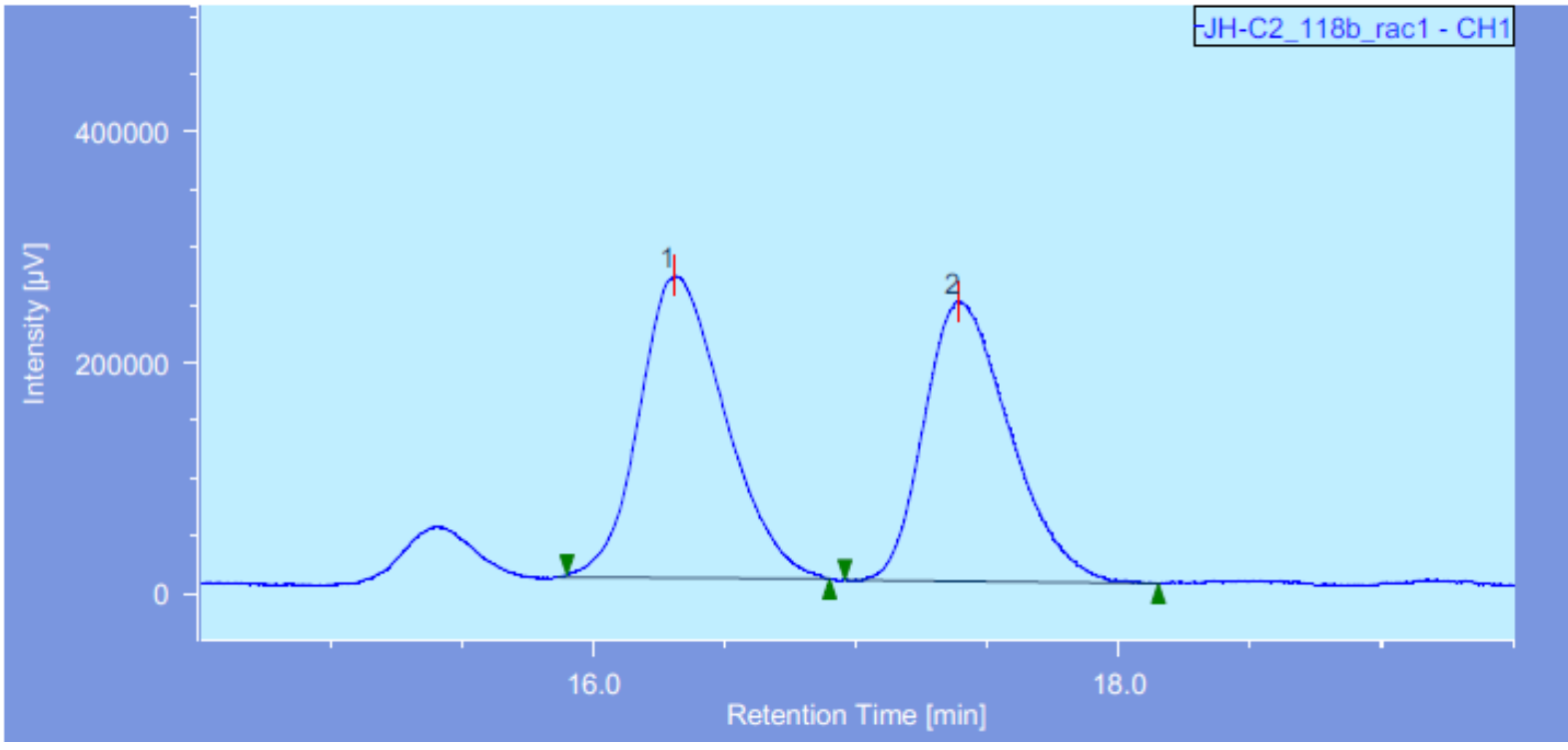

\begin{tabular}{|c|c|c|c|c|c|c|c|c|c|c|c|}
\hline Peak Name & $\mathrm{CH}$ & $t \mathbb{R}[\min ]$ & Area $[\mu \mathrm{V} \cdot \sec ]$ & Height $[\mu \mathrm{V}]$ & Area\% & Height\% & Quantity & NIP & Resolution & Symmetry Factor & Wanning \\
\hline & 1 & 16.30 & & 261000 & & & $\mathrm{~N} / \mathrm{A}$ & 12139 & 1.817 & & \\
\hline Unknown & 1 & $\frac{16.306}{17.392}$ & $\frac{3919468}{5497029}$ & $\frac{26000}{241499}$ & $\frac{13.83}{48.16}$ & $\frac{31.94 y}{48.060}$ & $\frac{N / A}{N / A}$ & $\frac{12139}{13278}$ & $\frac{1.81}{\mathrm{~N} / \mathrm{A}}$ & 1.213 & \\
\hline
\end{tabular}




\section{NMR Spectral Data}

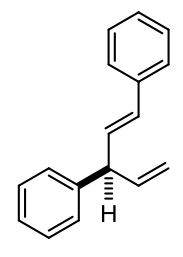

$3 a$

${ }^{1} \mathrm{H}-\mathrm{NMR}: 300 \mathrm{MHz}, \mathrm{CDCl}_{3}$

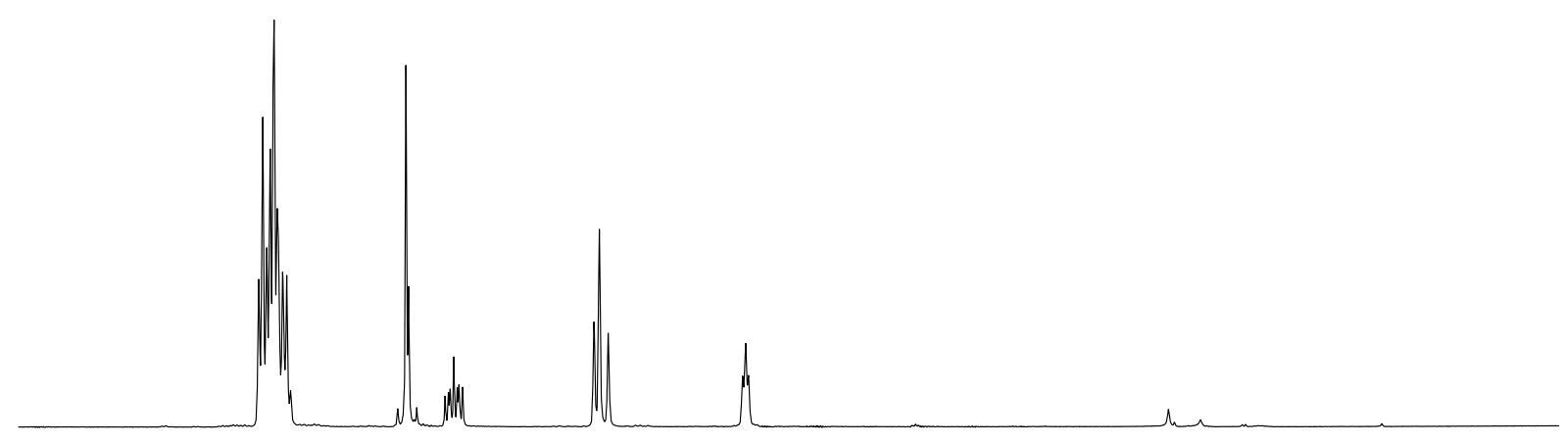

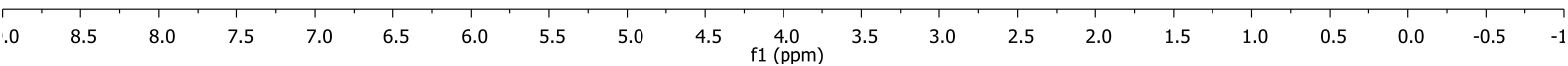

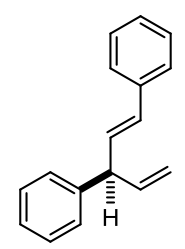

$3 a$

${ }^{13} \mathrm{C}-\mathrm{NMR}: 75 \mathrm{MHz}, \mathrm{CDCl}_{3}$

00

$\begin{array}{lllll}190 & 180 & 170 & 160 & 150\end{array}$

$140 \quad 130$ $120 \quad 110 \begin{gathered}100 \\ f 1(\mathrm{ppm})\end{gathered}$ 
S46

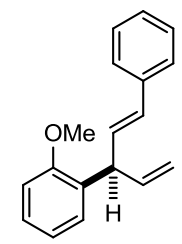

3b

${ }^{1} \mathrm{H}-\mathrm{NMR}: 400 \mathrm{MHz}, \mathrm{CDCl}_{3}$
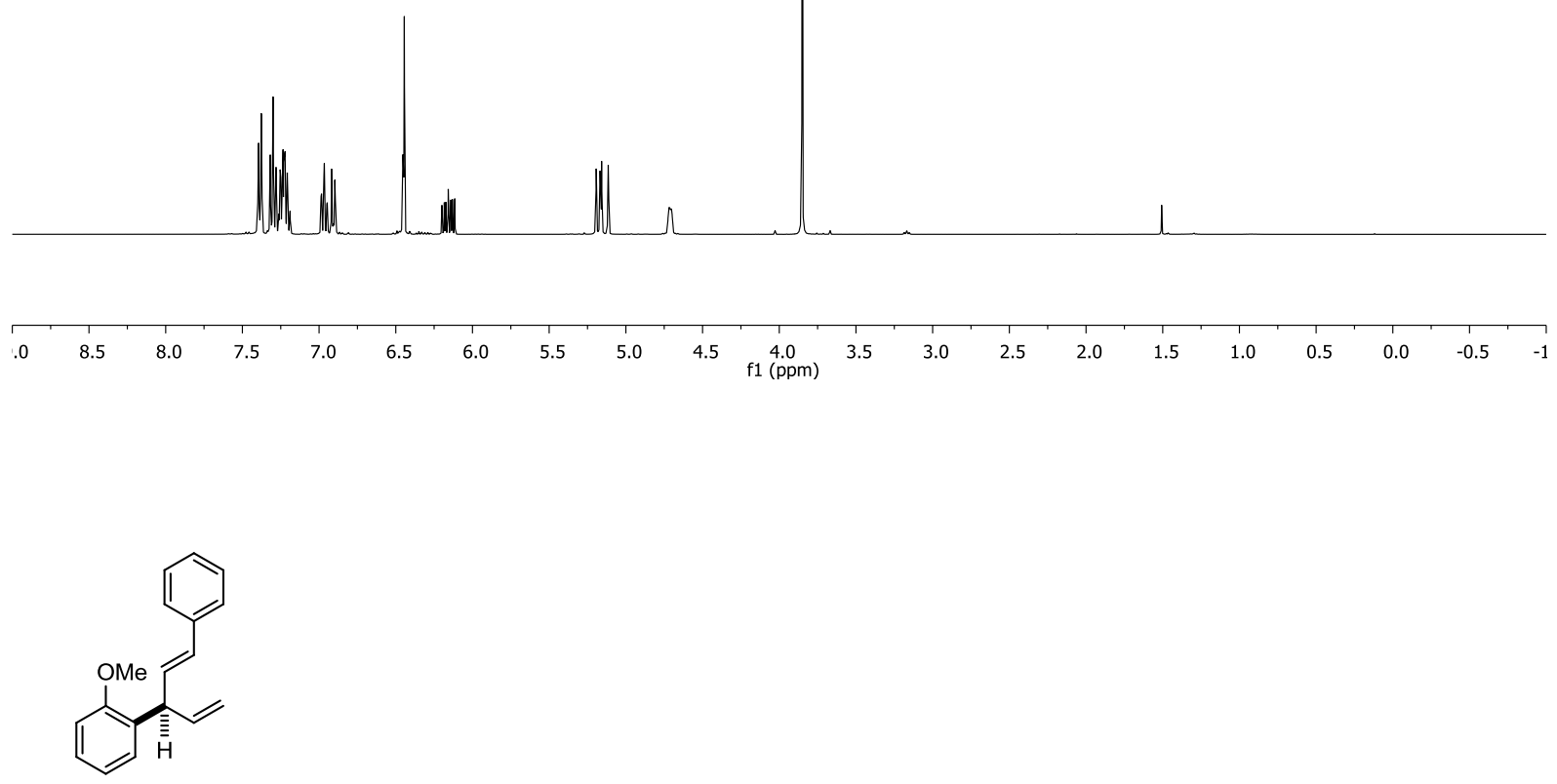

3b
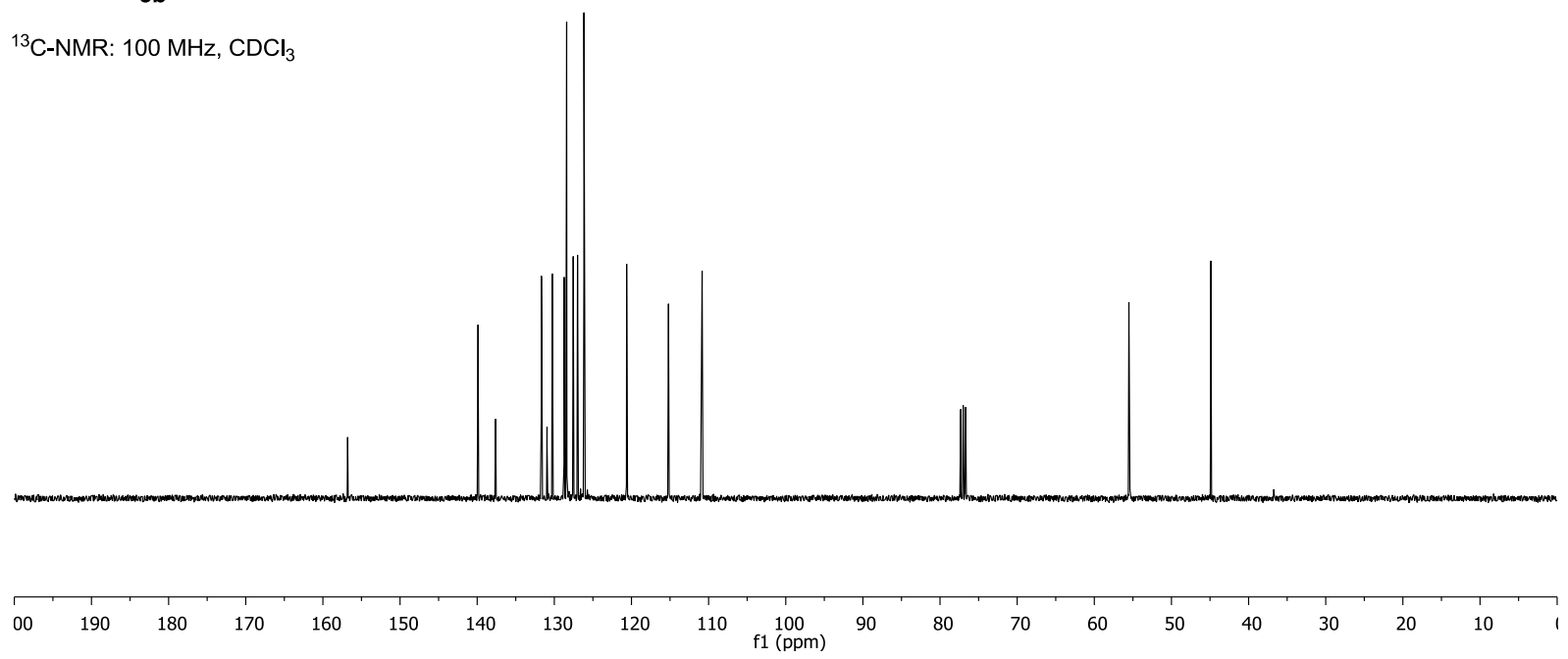


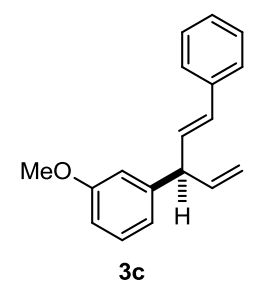

${ }^{1} \mathrm{H}-\mathrm{NMR}: 400 \mathrm{MHz}, \mathrm{CDCl}_{3}$
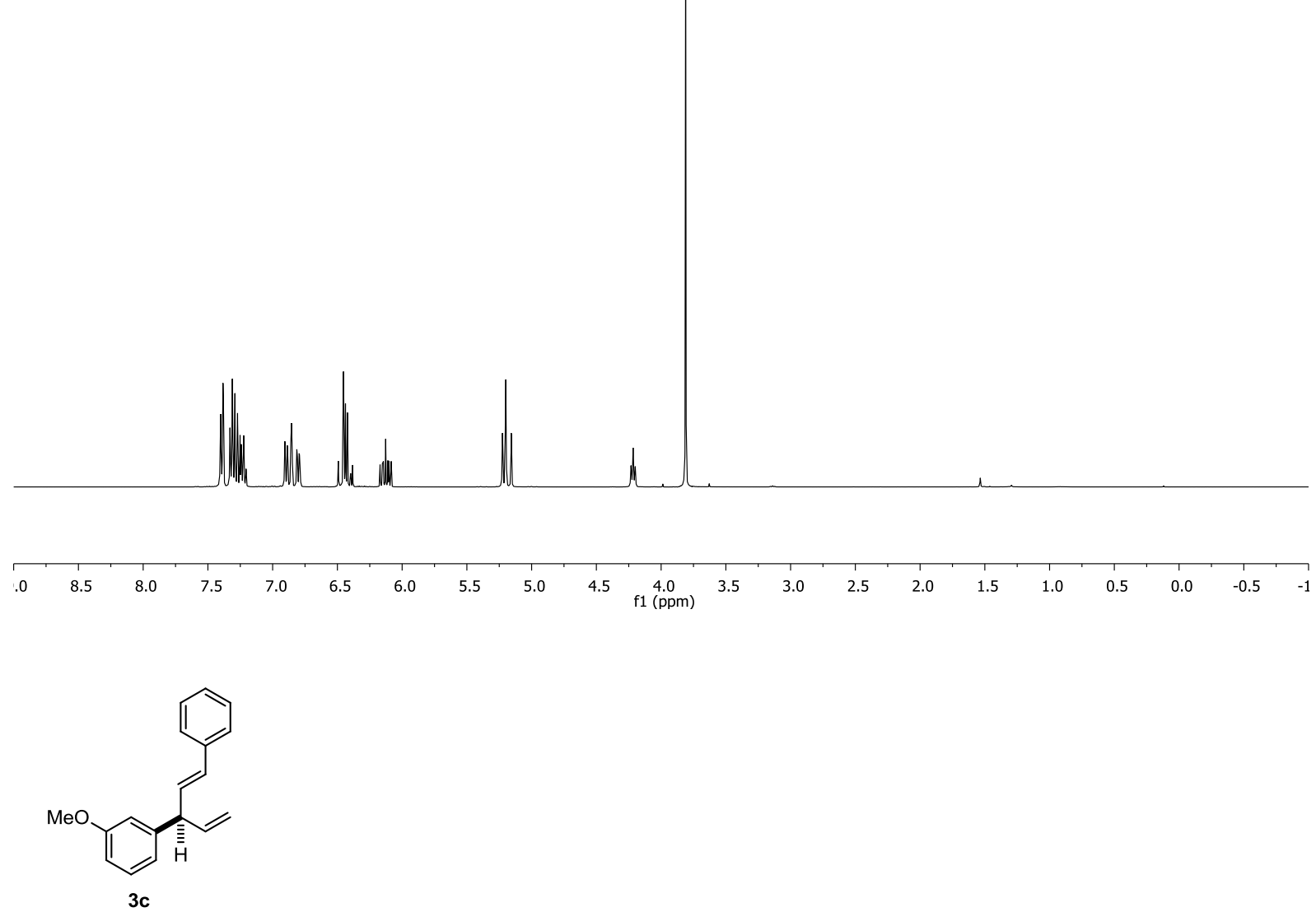

${ }^{13}$ C-NMR: $100 \mathrm{MHz}, \mathrm{CDCl}_{3}$
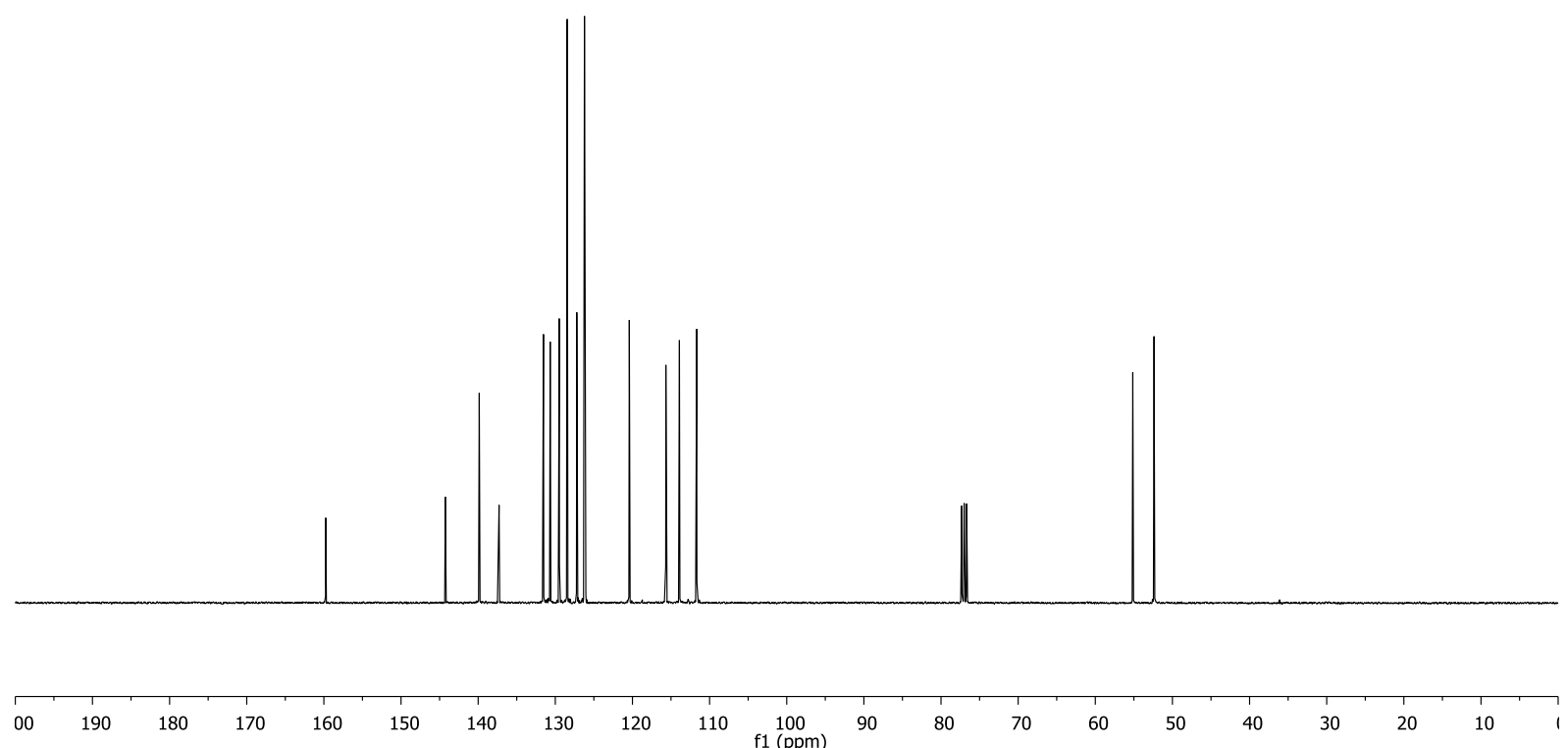


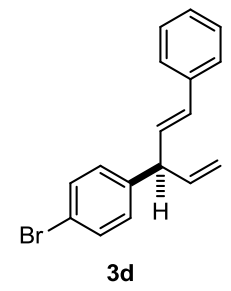

${ }^{1} \mathrm{H}-\mathrm{NMR}: 400 \mathrm{MHz}, \mathrm{CDCl}_{3}$
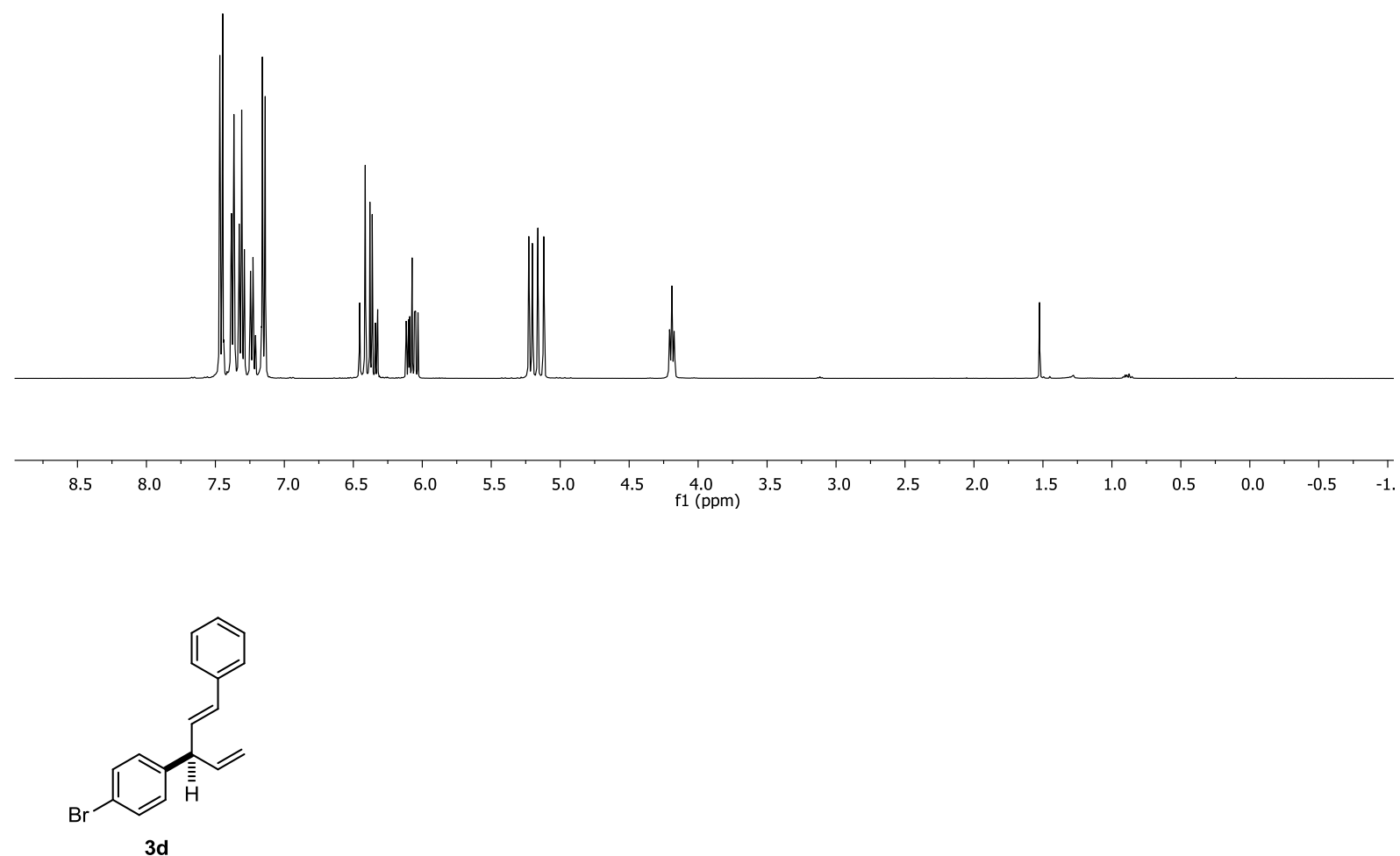

${ }^{13} \mathrm{C}-\mathrm{NMR}: 100 \mathrm{MHz}, \mathrm{CDCl}_{3}$

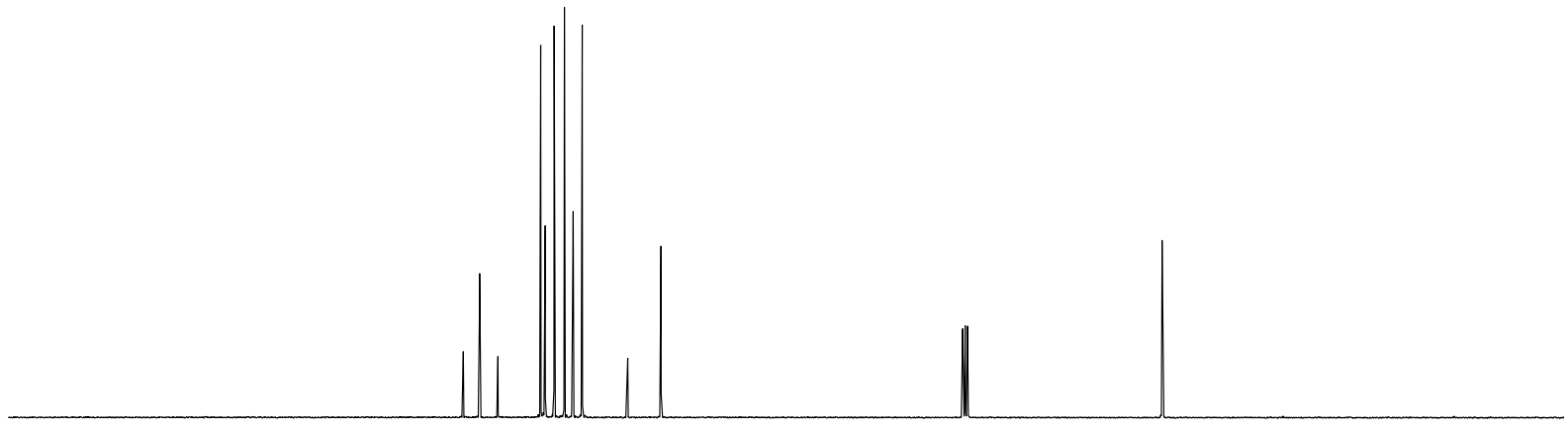

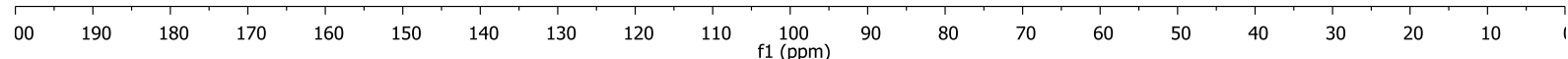




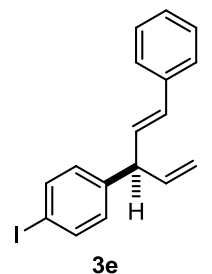

${ }^{1} \mathrm{H}-\mathrm{NMR}: 400 \mathrm{MHz}, \mathrm{CDCl}_{3}$
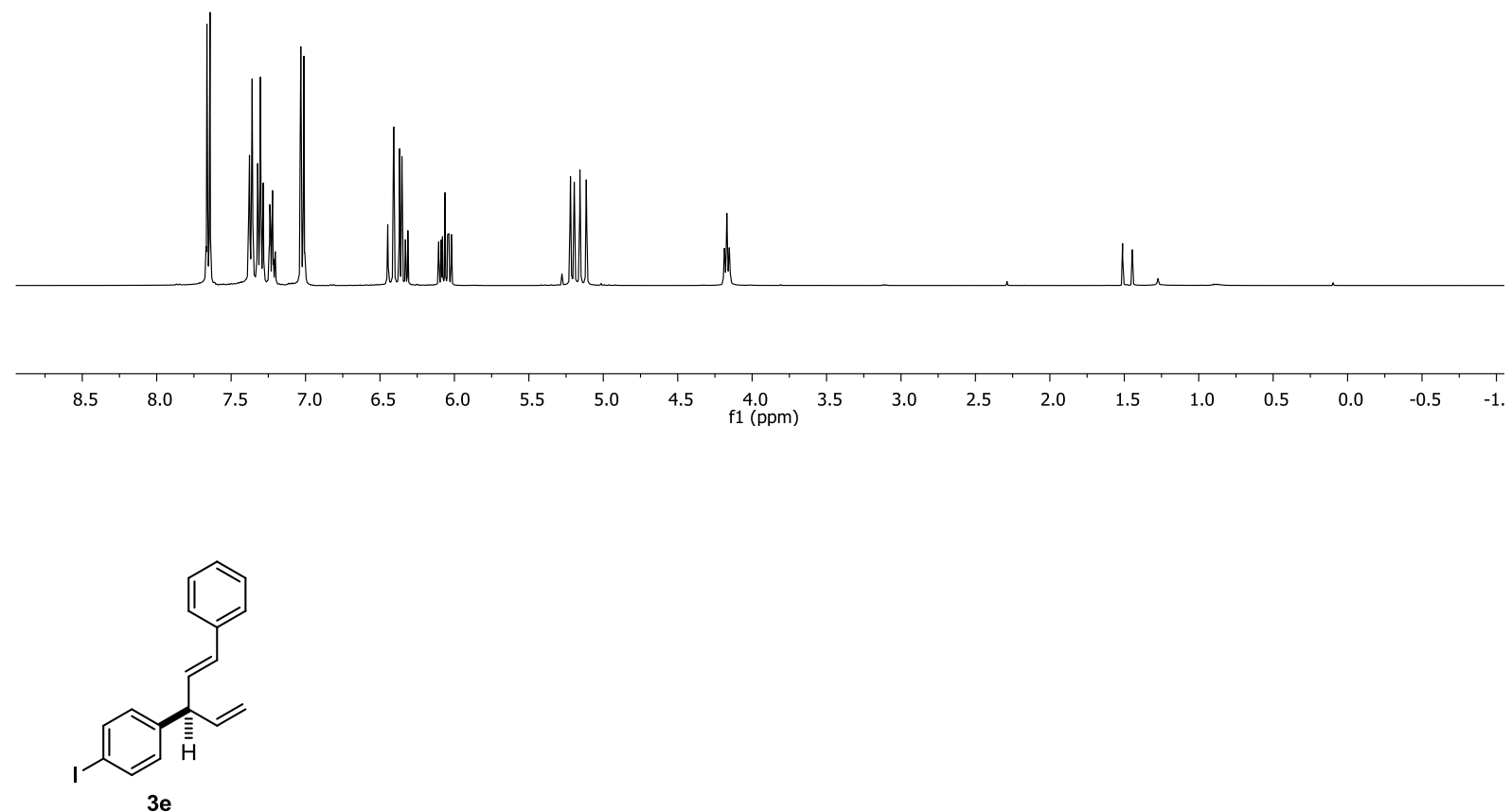

${ }^{13} \mathrm{C}$-NMR: $100 \mathrm{MHz}, \mathrm{CDCl}_{3}$
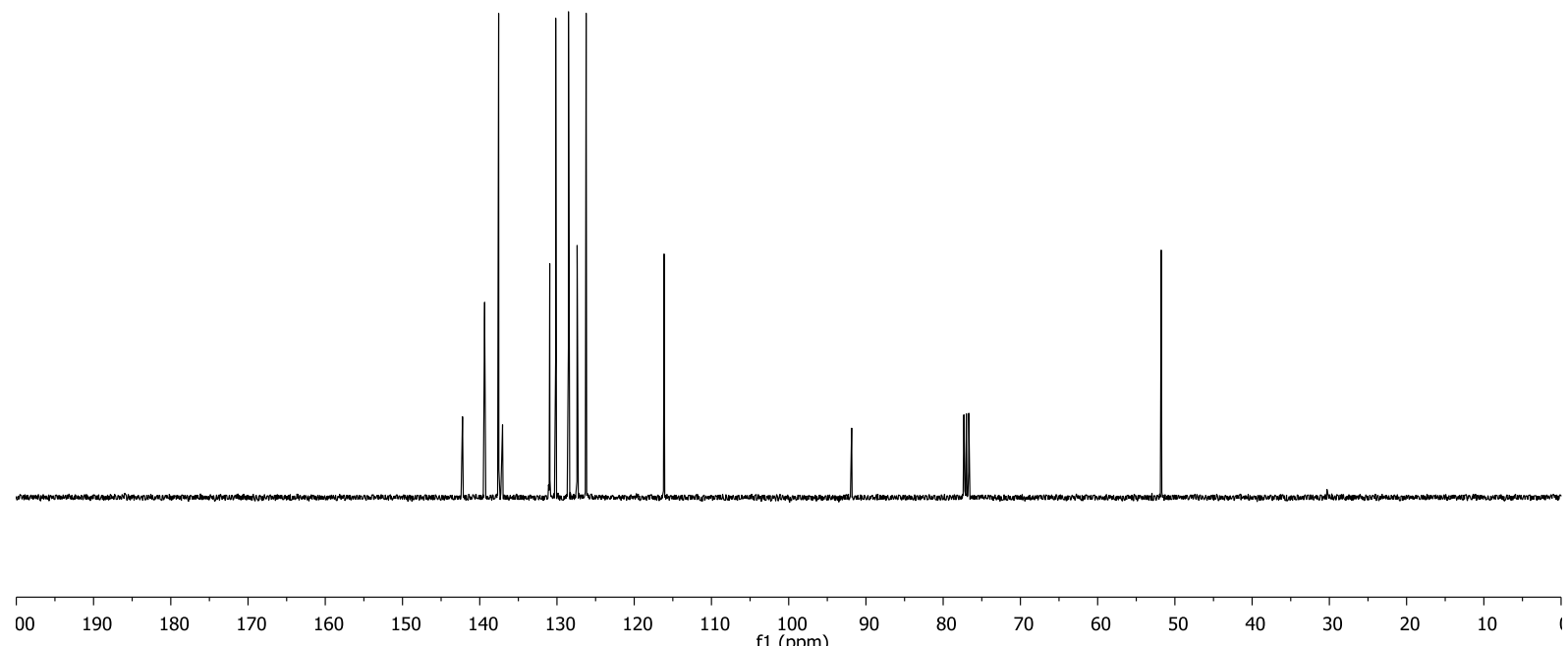


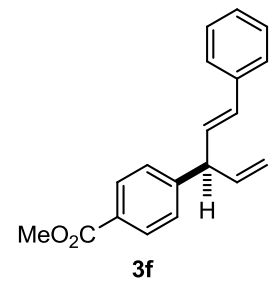

${ }^{1} \mathrm{H}-\mathrm{NMR}: 300 \mathrm{MHz}, \mathrm{CDCl}_{3}$

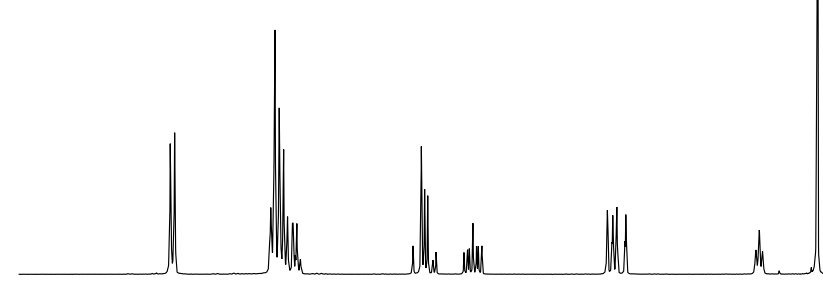

.0
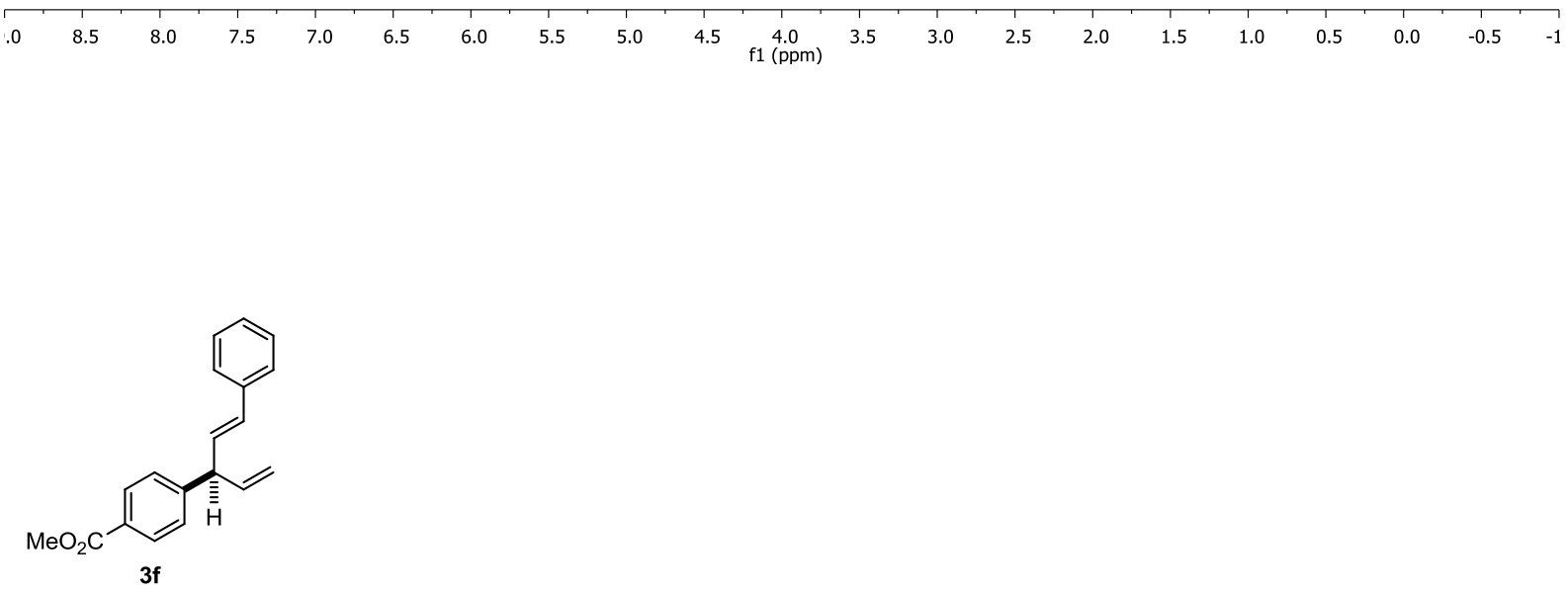

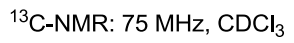
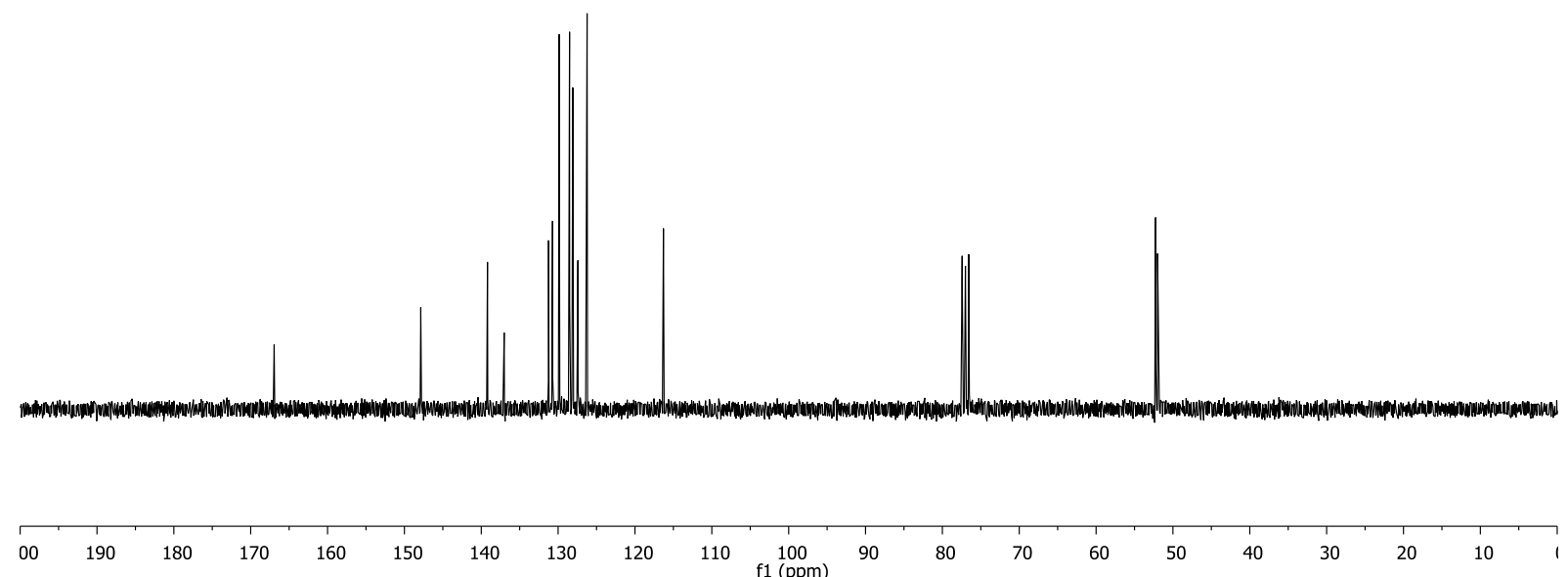


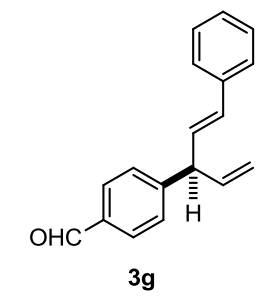

${ }^{1} \mathrm{H}-\mathrm{NMR}: 400 \mathrm{MHz}, \mathrm{CDCl}_{3}$

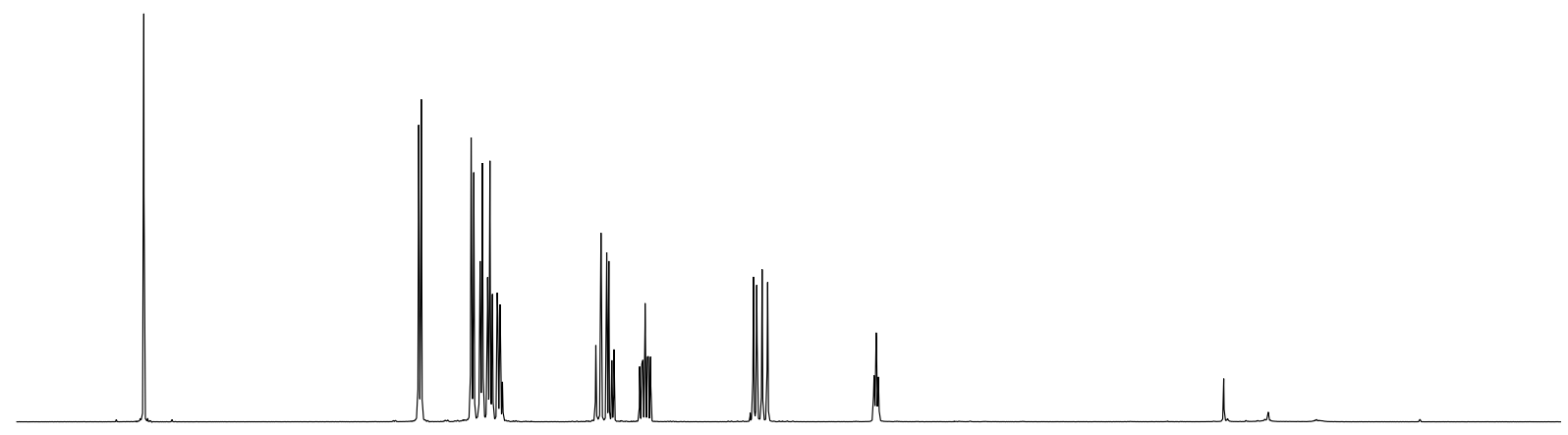

$\begin{array}{lllllllllllllllllllllllll}1.0 & 10.5 & 10.0 & 9.5 & 9.0 & 8.5 & 8.0 & 7.5 & 7.0 & 6.5 & 6.0 & 5.5 & 5.0 & 4.5 & 4.0 & 3.5 & 3.0 & 2.5 & 2.0 & 1.5 & 1.0 & 0.5 & 0.0 & -0.5 & -1\end{array}$

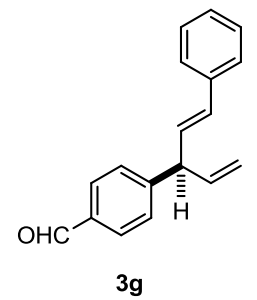

${ }^{13} \mathrm{C}-\mathrm{NMR}: 100 \mathrm{MHz}, \mathrm{CDCl}_{3}$

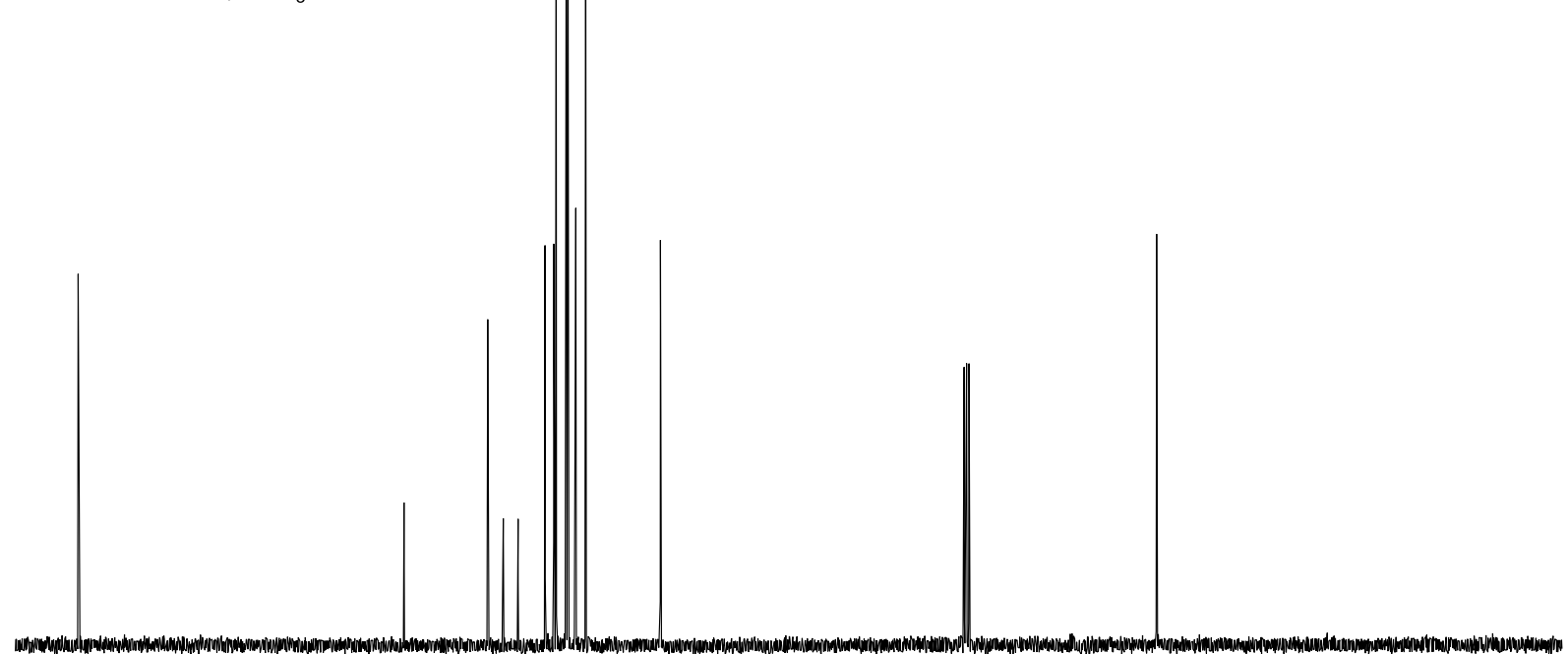

$\begin{array}{llllllllllllllllllllllll}00 & 190 & 180 & 170 & 160 & 150 & 140 & 130 & 120 & 110 & 100 & 90 & 80 & 70 & 60 & 50 & 40 & 30 & 20 & 10 & 10\end{array}$ 


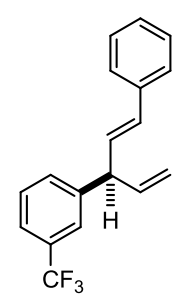

$3 \mathbf{h}$

${ }^{1} \mathrm{H}-\mathrm{NMR}: 400 \mathrm{MHz}, \mathrm{CDCl}_{3}$

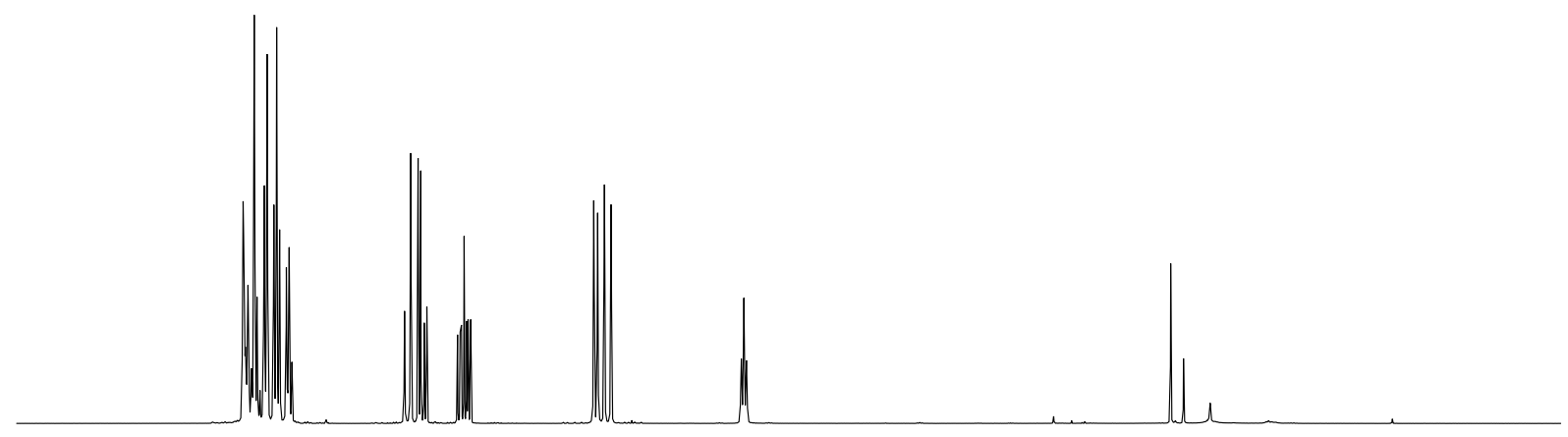

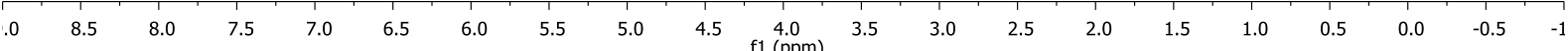<smiles>C=C[C@H](/C=C/c1ccccc1)c1cccc(C(F)(F)F)c1</smiles>

3h

${ }^{13} \mathrm{C}-\mathrm{NMR}: 100 \mathrm{MHz}, \mathrm{CDCl}_{3}$

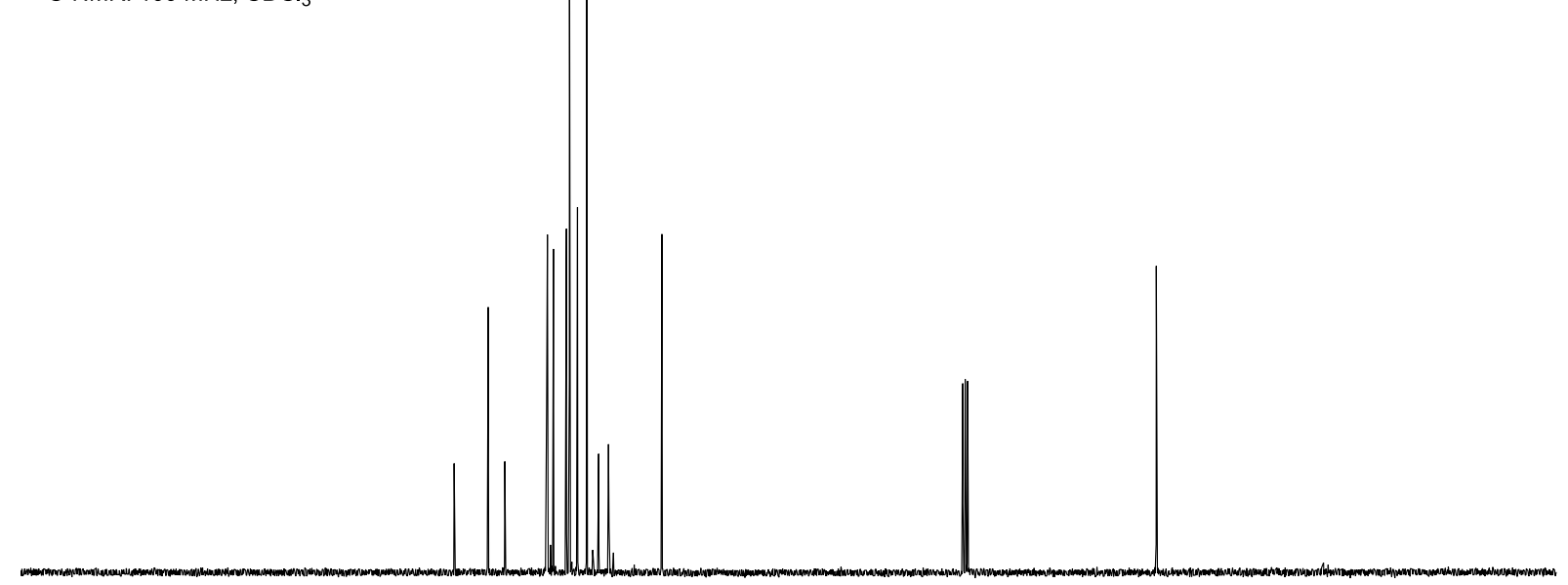

00

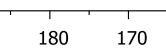

$160 \quad 150$ $\begin{array}{lllll}140 & 130 & 120 & 110 & \begin{array}{c}100 \\ \mathrm{f} 1(\mathrm{ppm})\end{array}\end{array}$

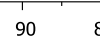

70
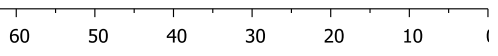


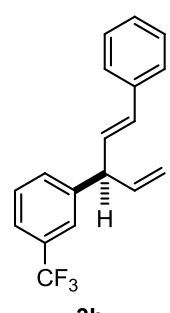

$3 \mathrm{~h}$

${ }^{19}$ F-NMR: $376 \mathrm{MHz}, \mathrm{CDCl}_{3}$

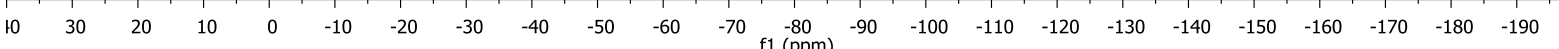

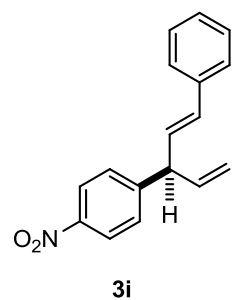

${ }^{1} \mathrm{H}-\mathrm{NMR}: 400 \mathrm{MHz}, \mathrm{CDCl}_{3}$
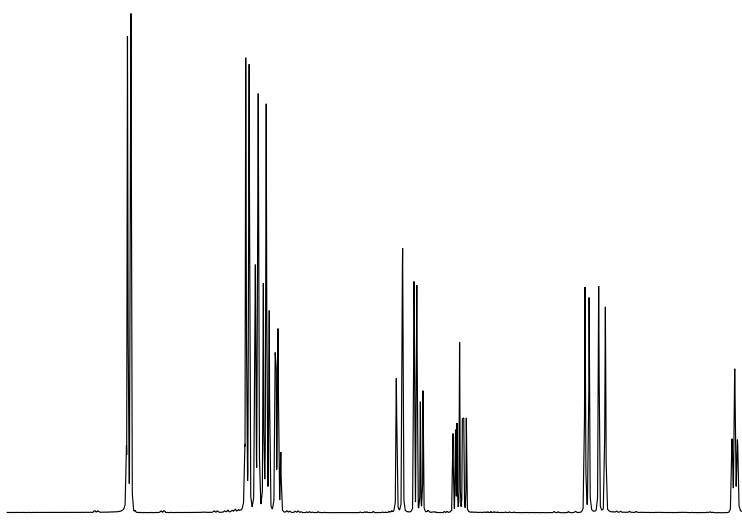

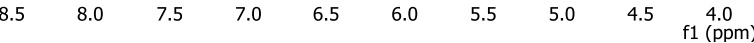

$3.5 \quad 3.0$

2.5

$\begin{array}{llllll}1.5 & 1.0 & 0.5 & 0.0 & -0.5 & -1\end{array}$ 
S54
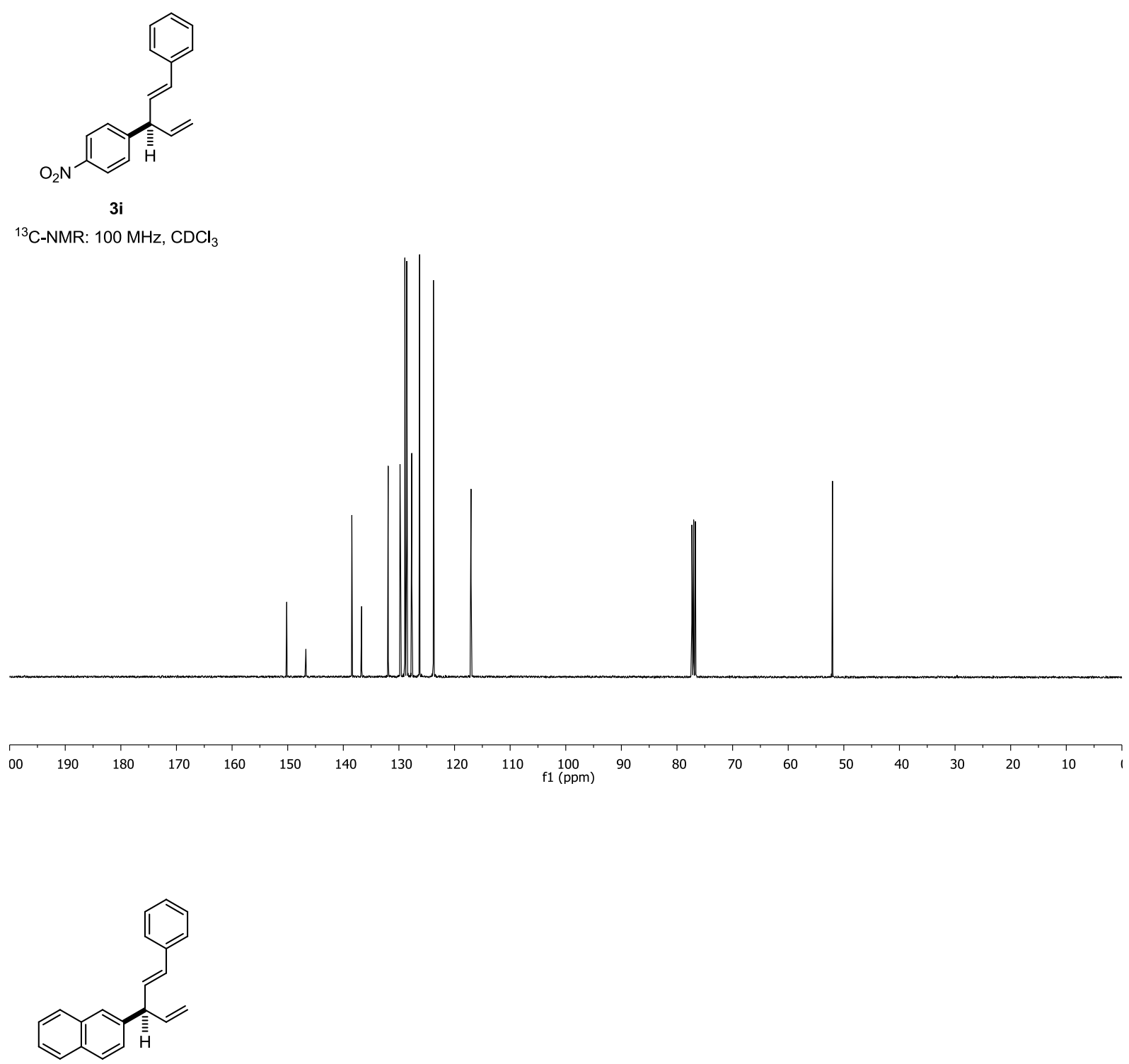

3j

${ }^{1} \mathrm{H}-\mathrm{NMR}: 400 \mathrm{MHz}, \mathrm{CDCl}_{3}$
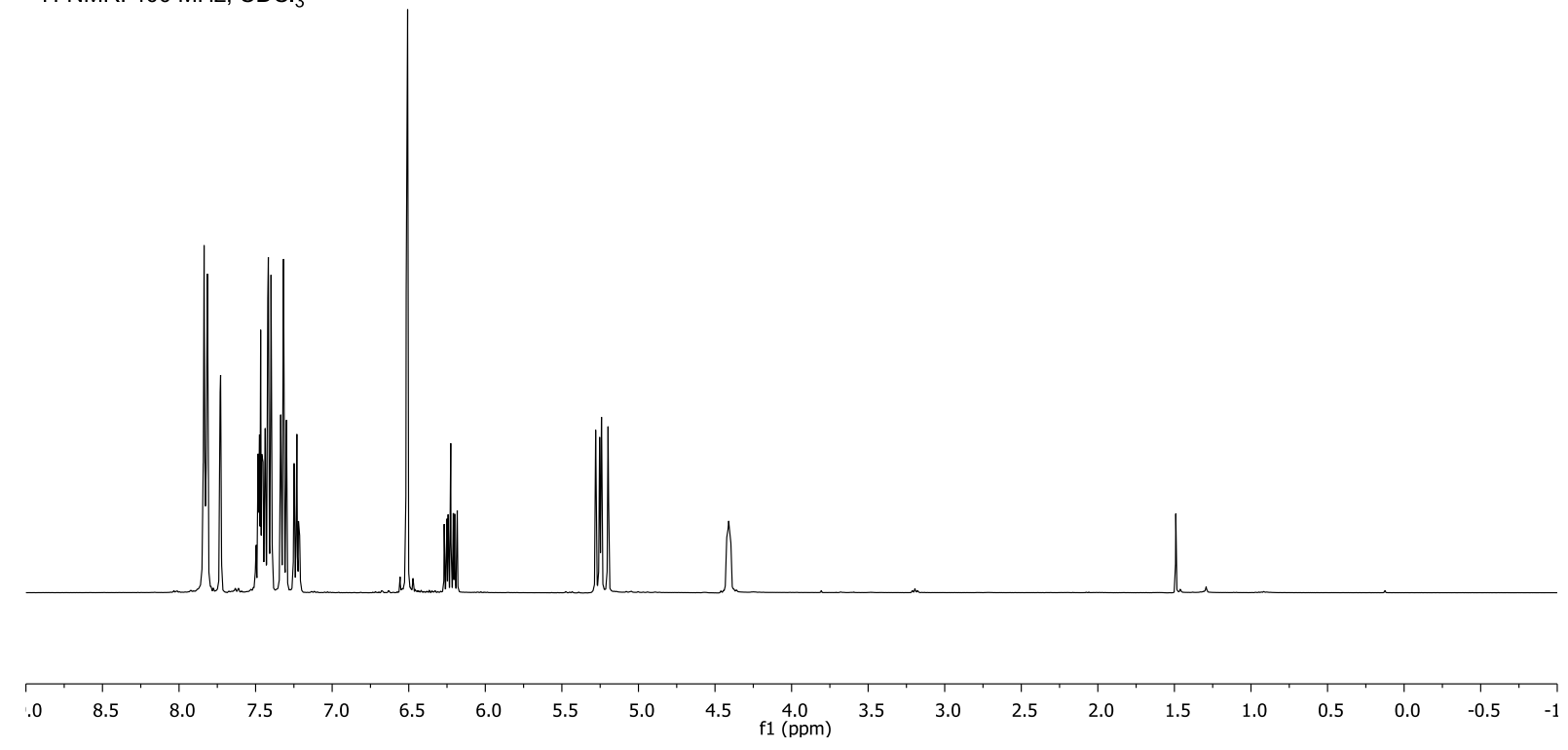
S55
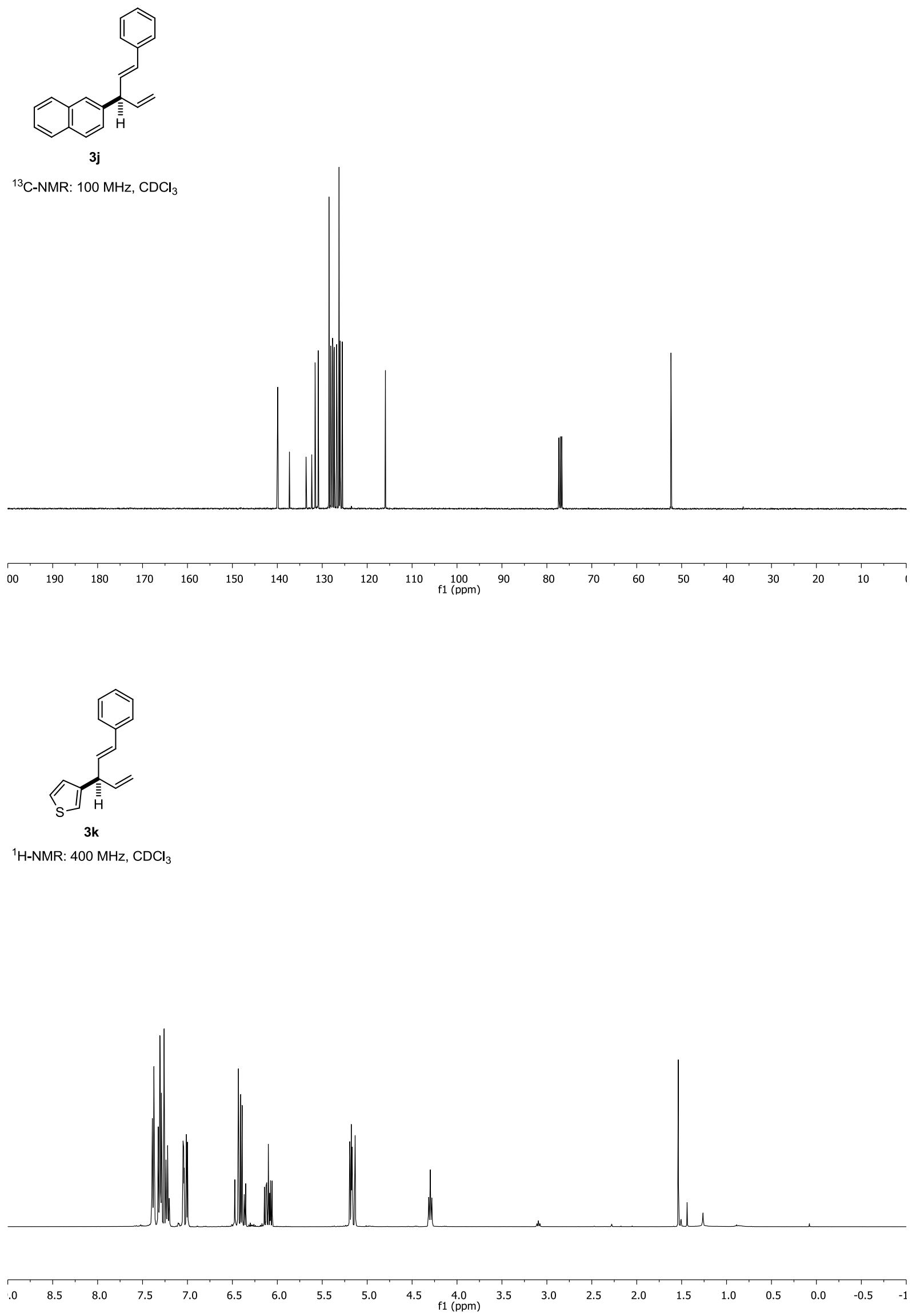
S56

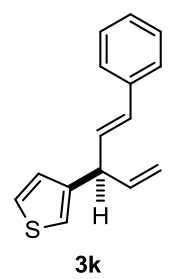

${ }^{13} \mathrm{C}-\mathrm{NMR}: 100 \mathrm{MHz}, \mathrm{CDCl}_{3}$
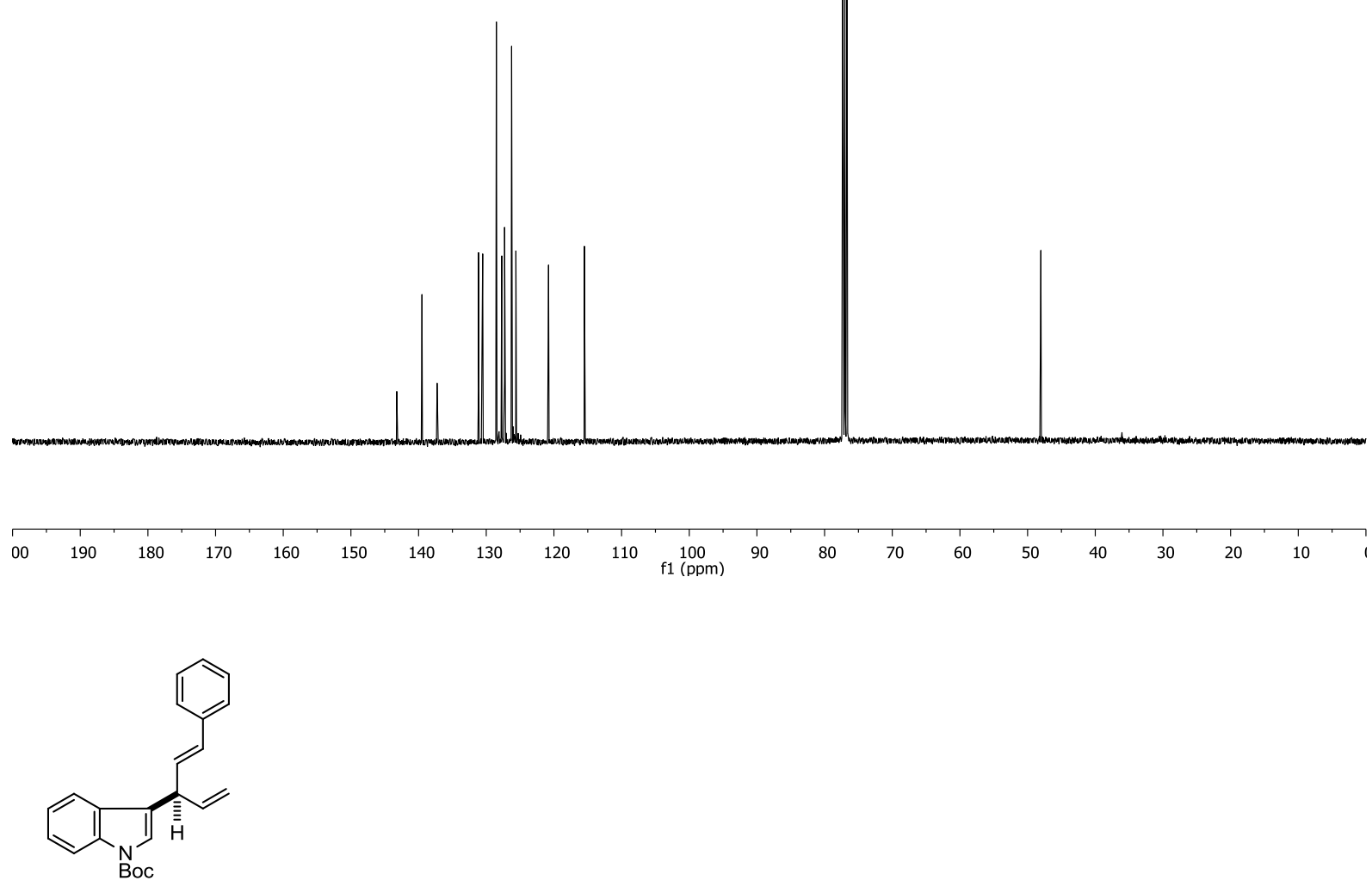

31

${ }^{1} \mathrm{H}-\mathrm{NMR}: 400 \mathrm{MHz}, \mathrm{CDCl}_{3}$
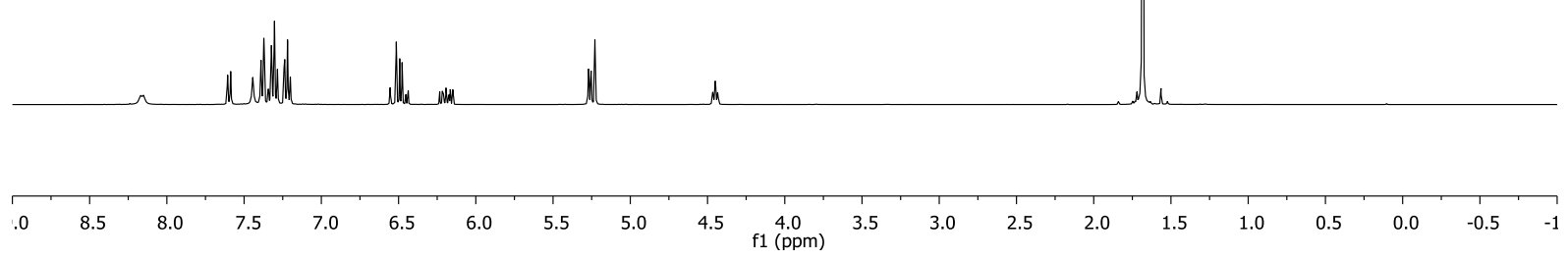
S57

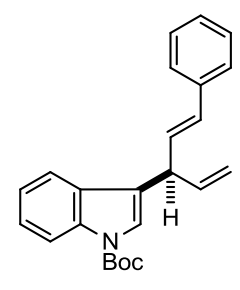

3I

${ }^{13} \mathrm{C}-\mathrm{NMR}: 100 \mathrm{MHz}, \mathrm{CDCl}_{3}$
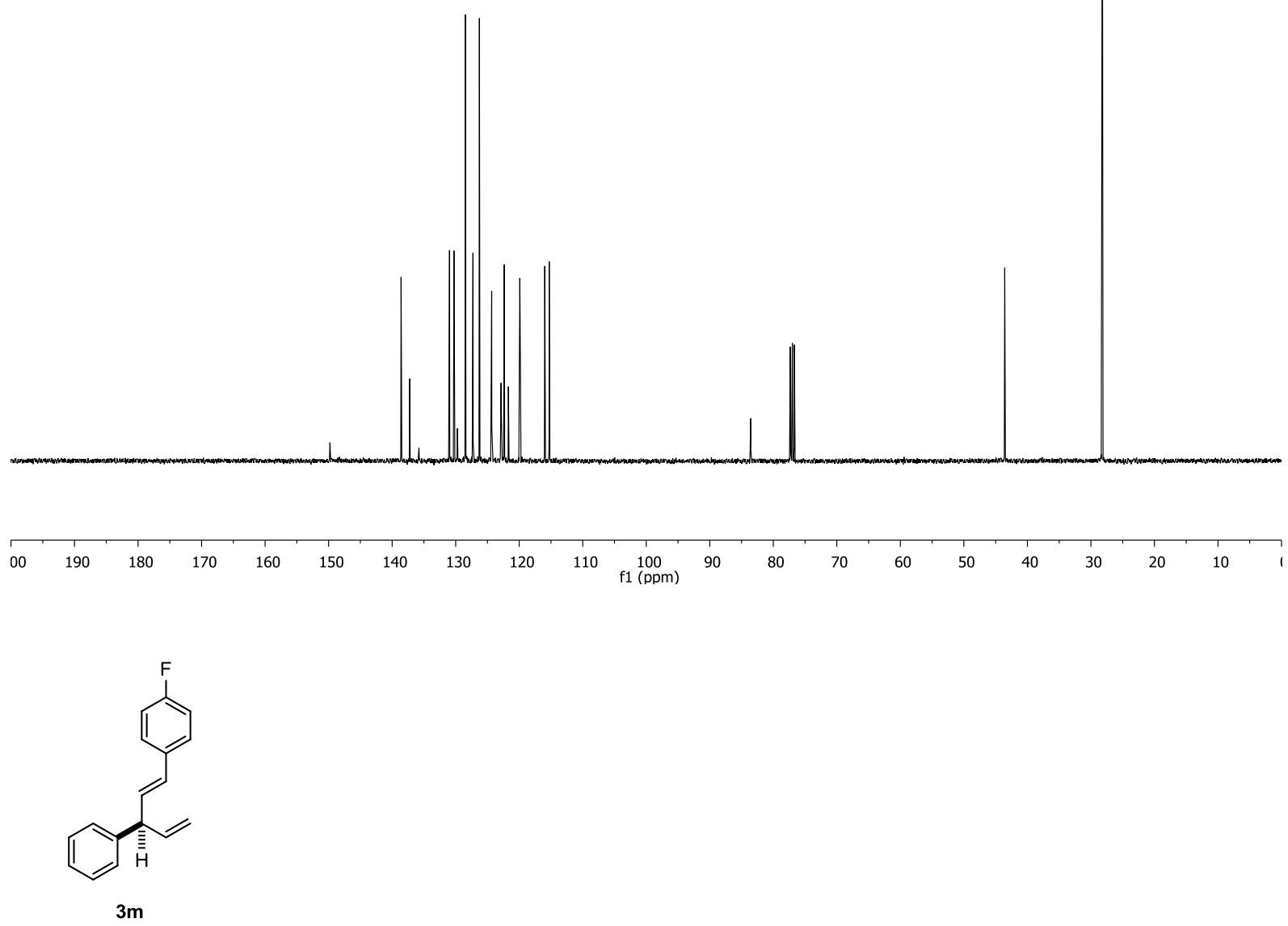

${ }^{1} \mathrm{H}-\mathrm{NMR}: 400 \mathrm{MHz}, \mathrm{CDCl}_{3}$
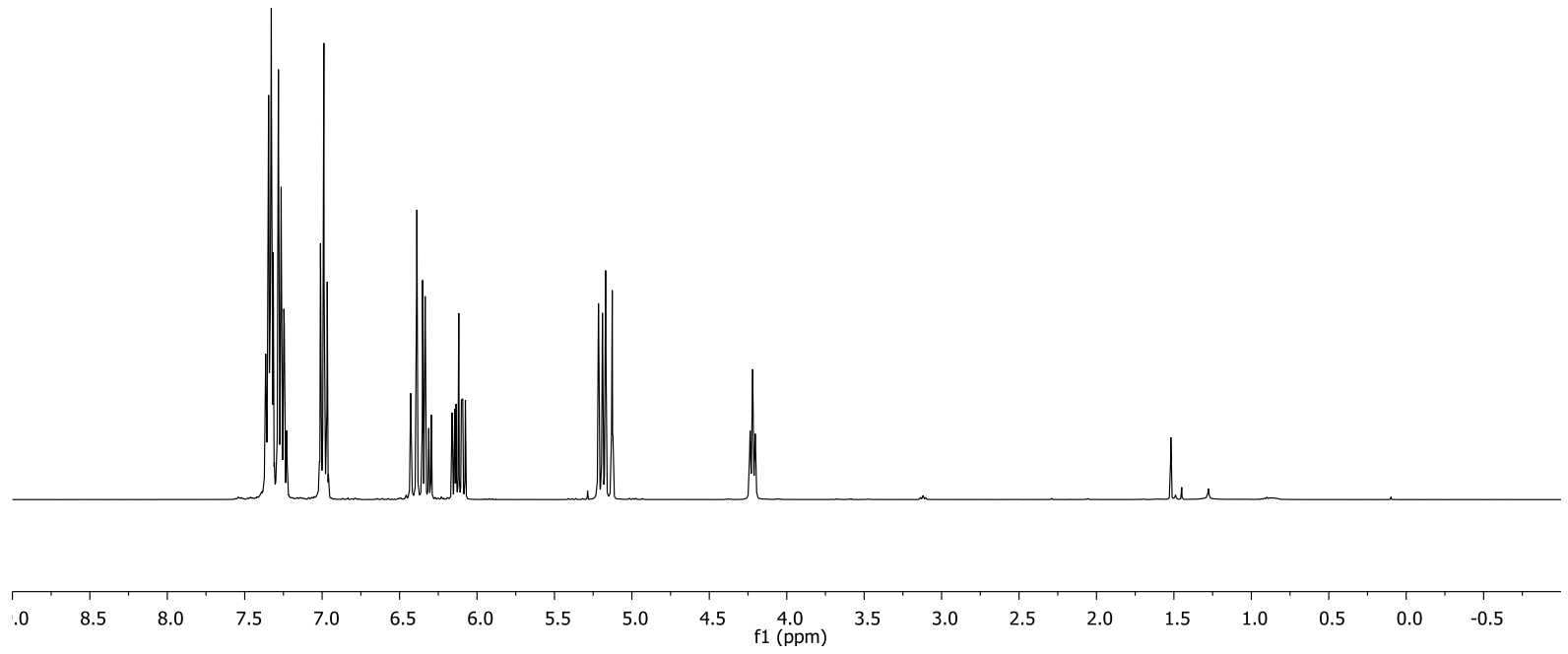
S58
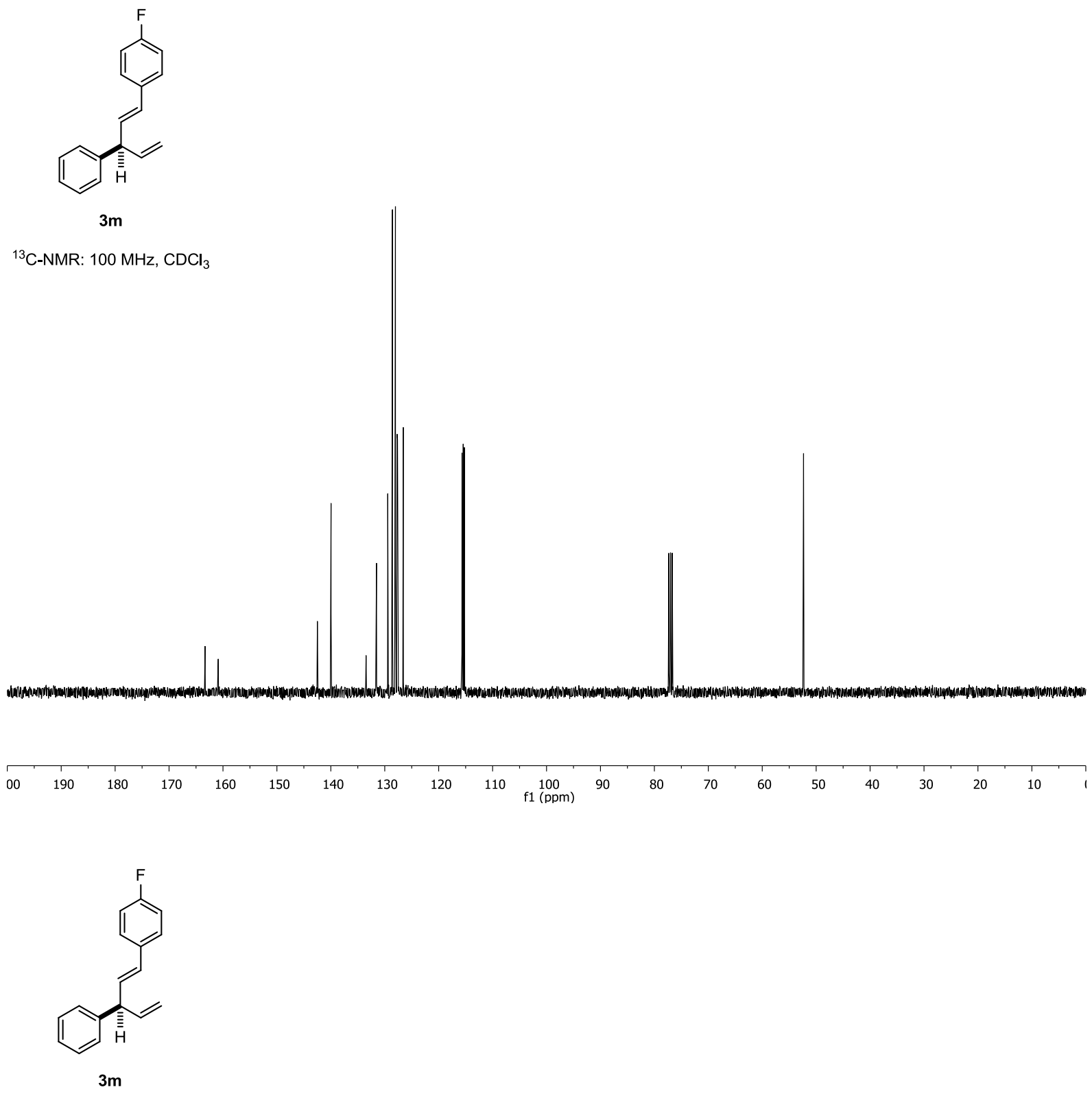

${ }^{19}$ F-NMR: $376 \mathrm{MHz}, \mathrm{CDCl}_{3}$

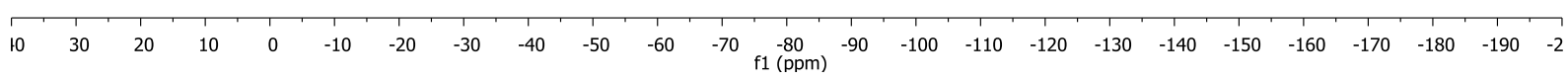




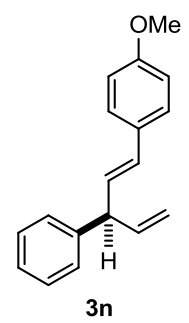

${ }^{1} \mathrm{H}-\mathrm{NMR}: 400 \mathrm{MHz}, \mathrm{CDCl}_{3}$
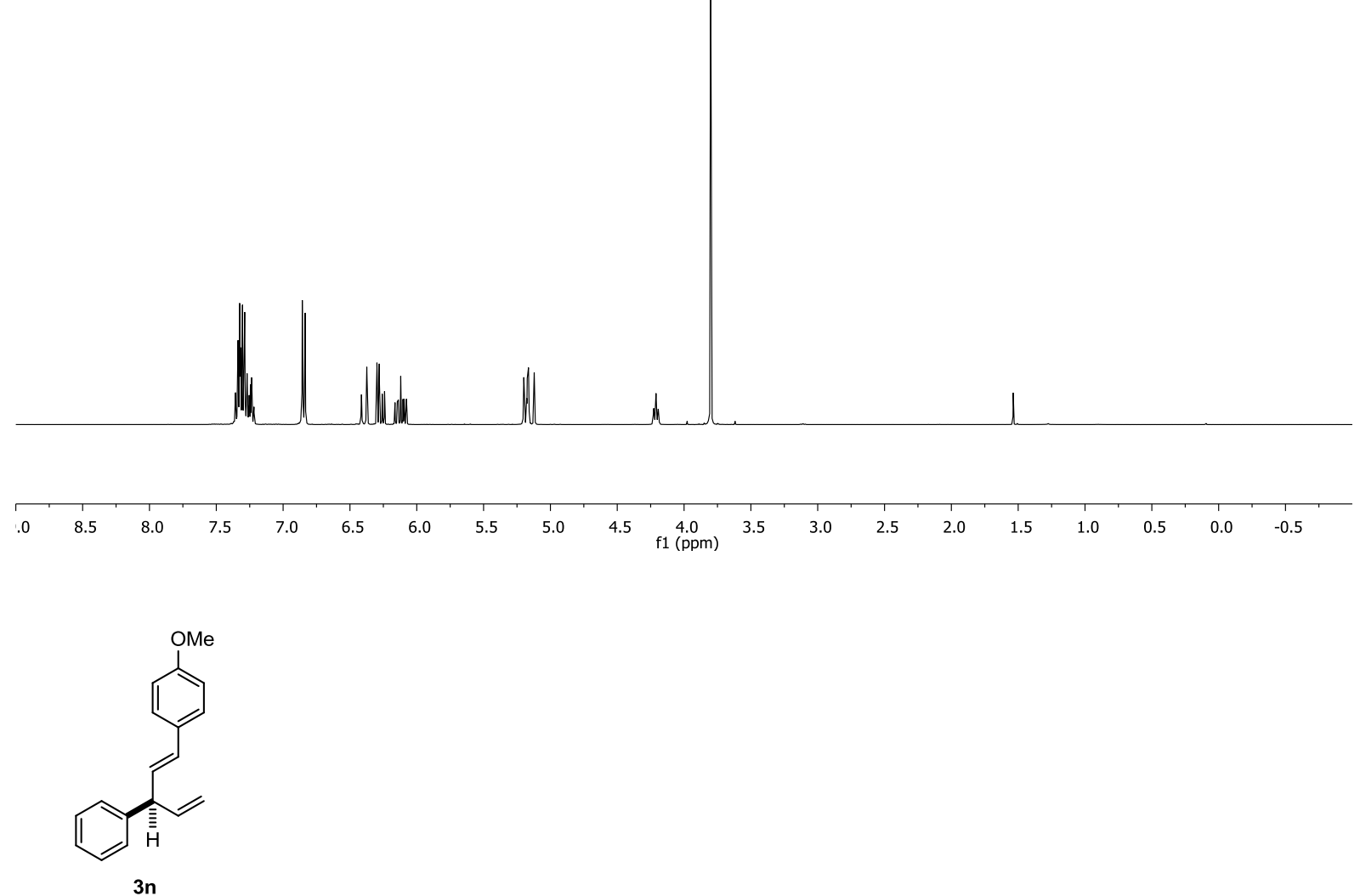

${ }^{13} \mathrm{C}-\mathrm{NMR}: 100 \mathrm{MHz}, \mathrm{CDCl}_{3}$

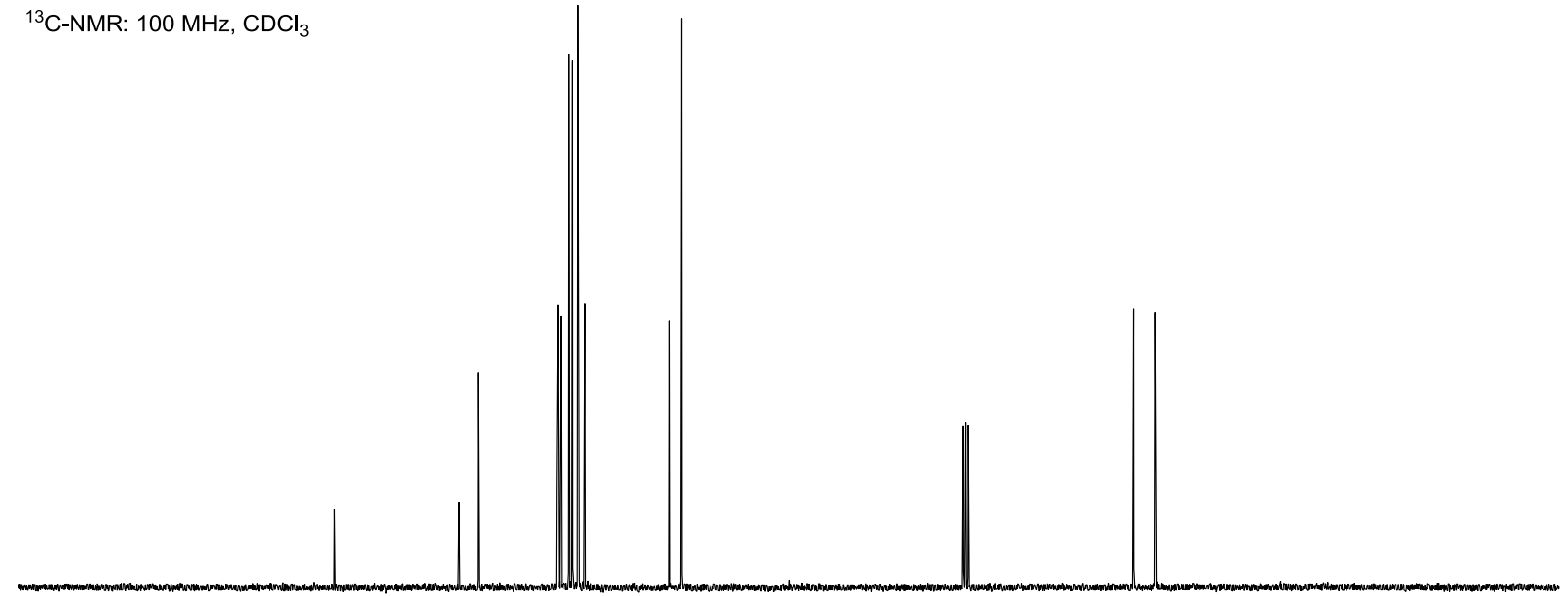

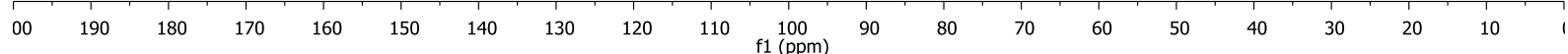


S60

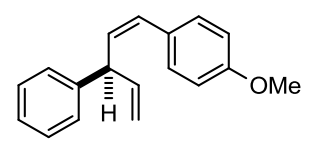

30

${ }^{1} \mathrm{H}-\mathrm{NMR}: 400 \mathrm{MHz}, \mathrm{CDCl}_{3}$
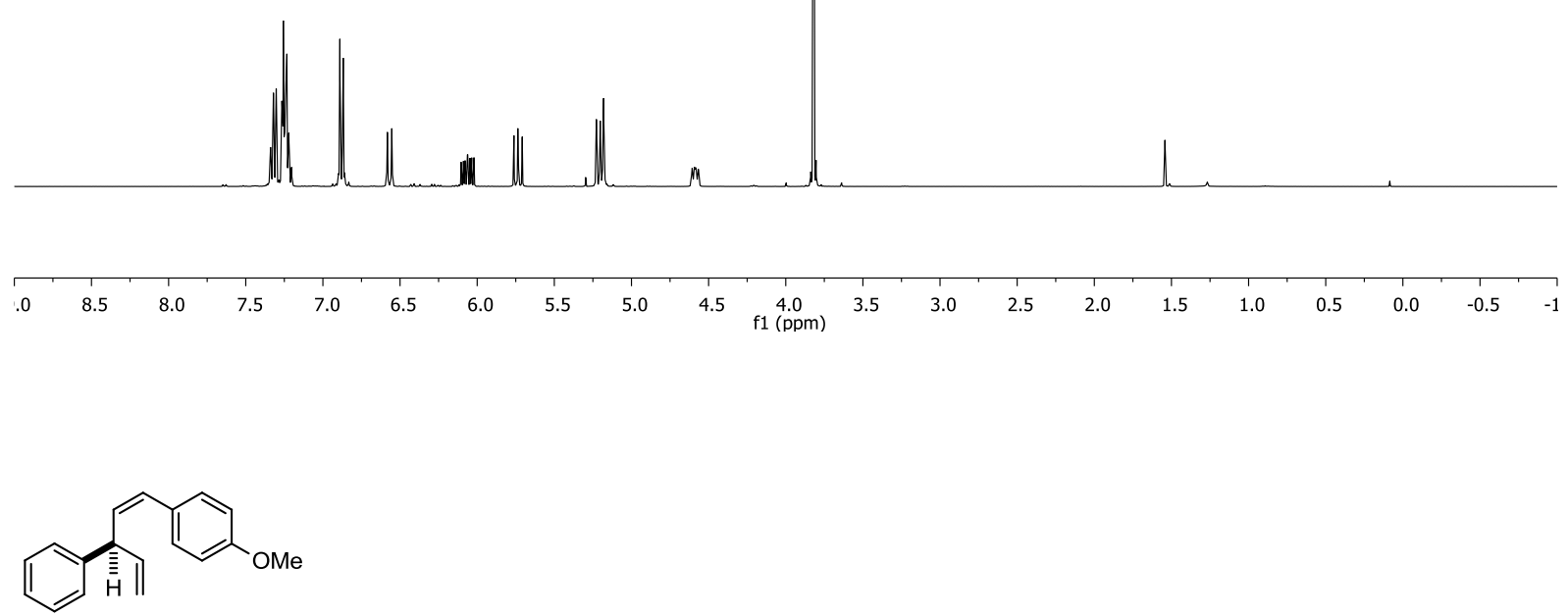

30

${ }^{13} \mathrm{C}-\mathrm{NMR}: 100 \mathrm{MHz}, \mathrm{CDCl}_{3}$
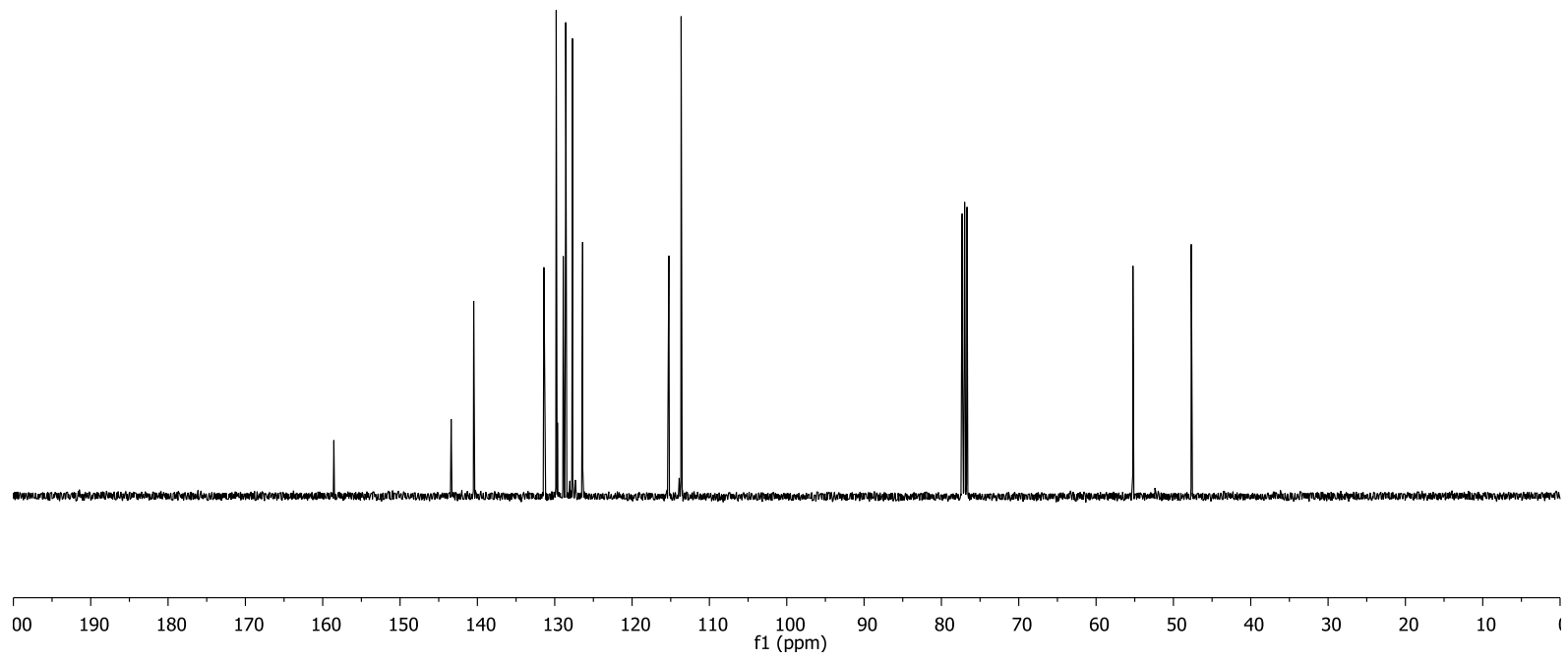


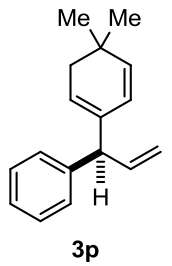

${ }^{1} \mathrm{H}-\mathrm{NMR}: 400 \mathrm{MHz}, \mathrm{CDCl}_{3}$
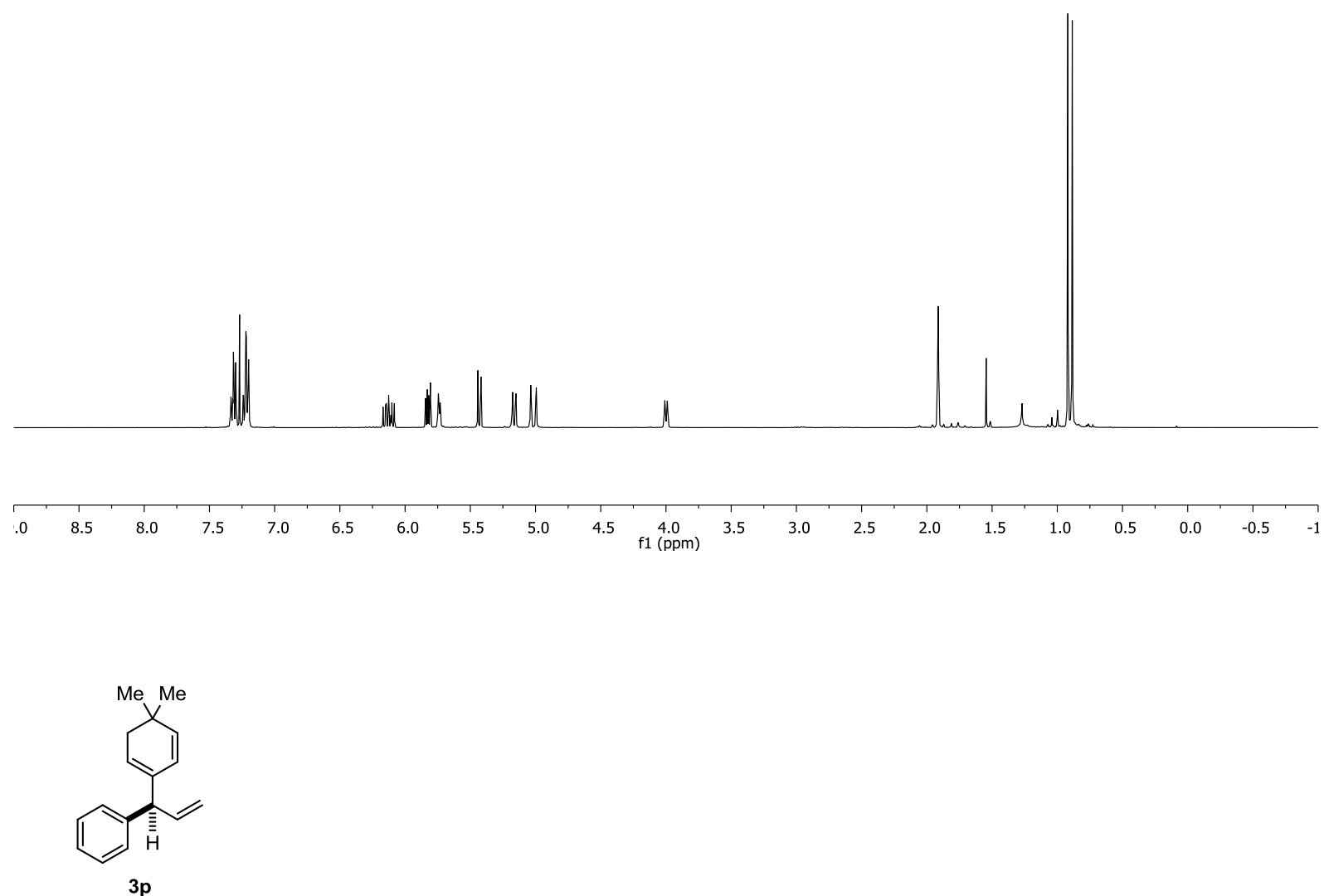

${ }^{13} \mathrm{C}-\mathrm{NMR}: 100 \mathrm{MHz}, \mathrm{CDCl}_{3}$

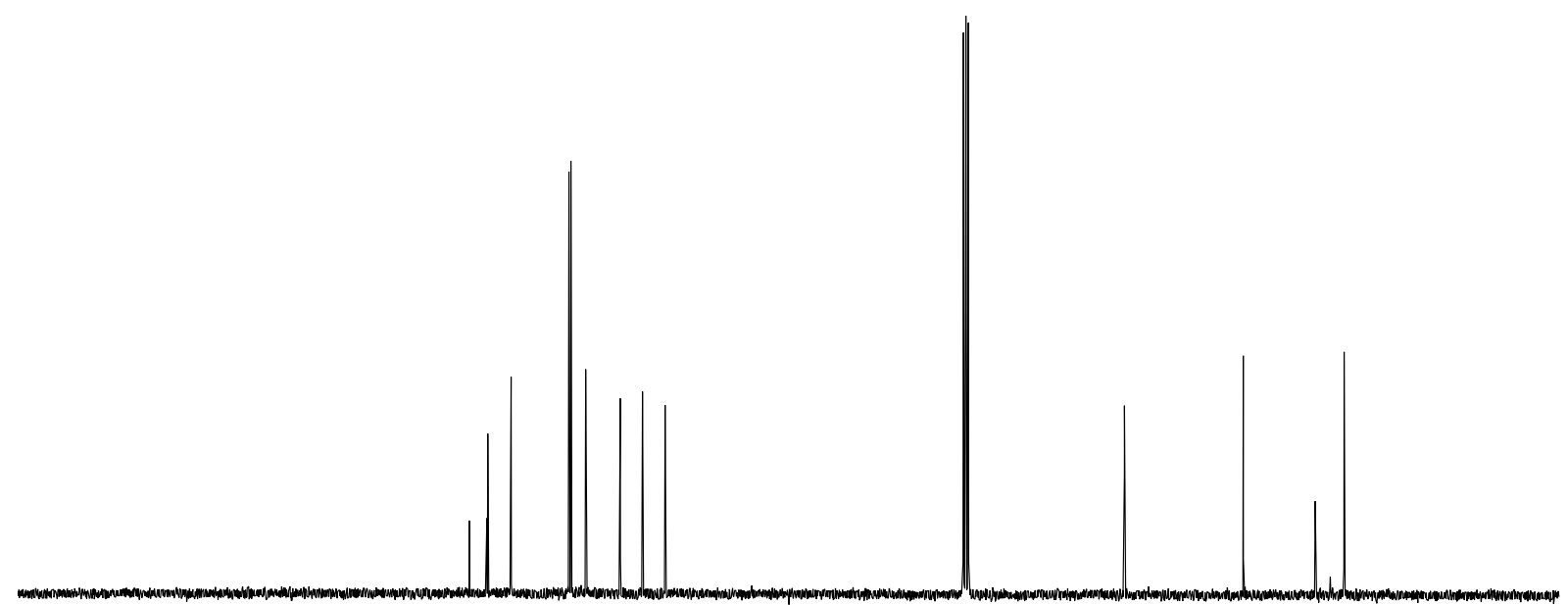


S62

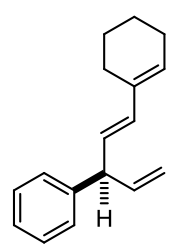

$3 q$

${ }^{1} \mathrm{H}-\mathrm{NMR}: 400 \mathrm{MHz}, \mathrm{CDCl}_{3}$
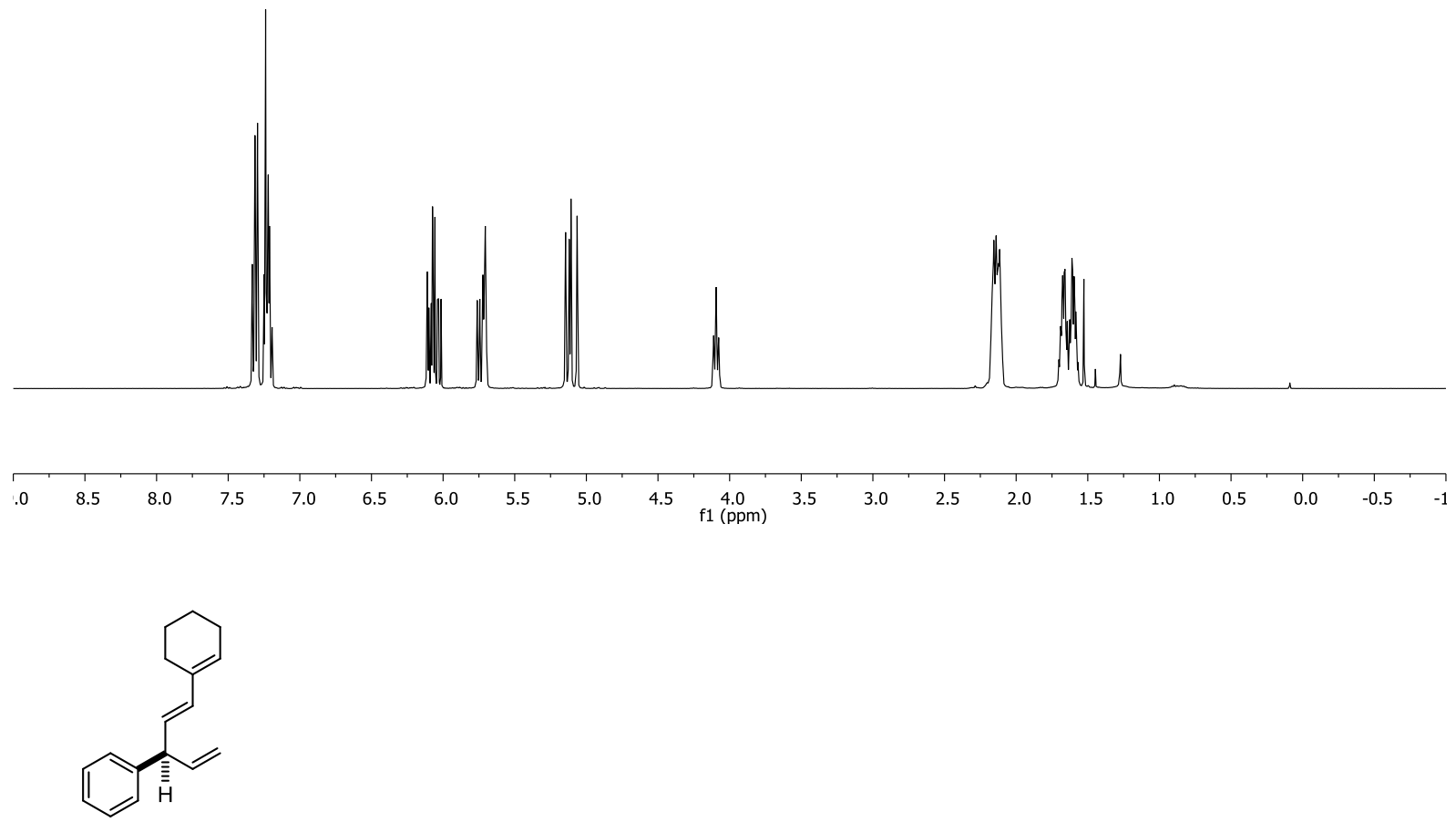

$3 q$

${ }^{13} \mathrm{C}-\mathrm{NMR}: 100 \mathrm{MHz}, \mathrm{CDCl}_{3}$

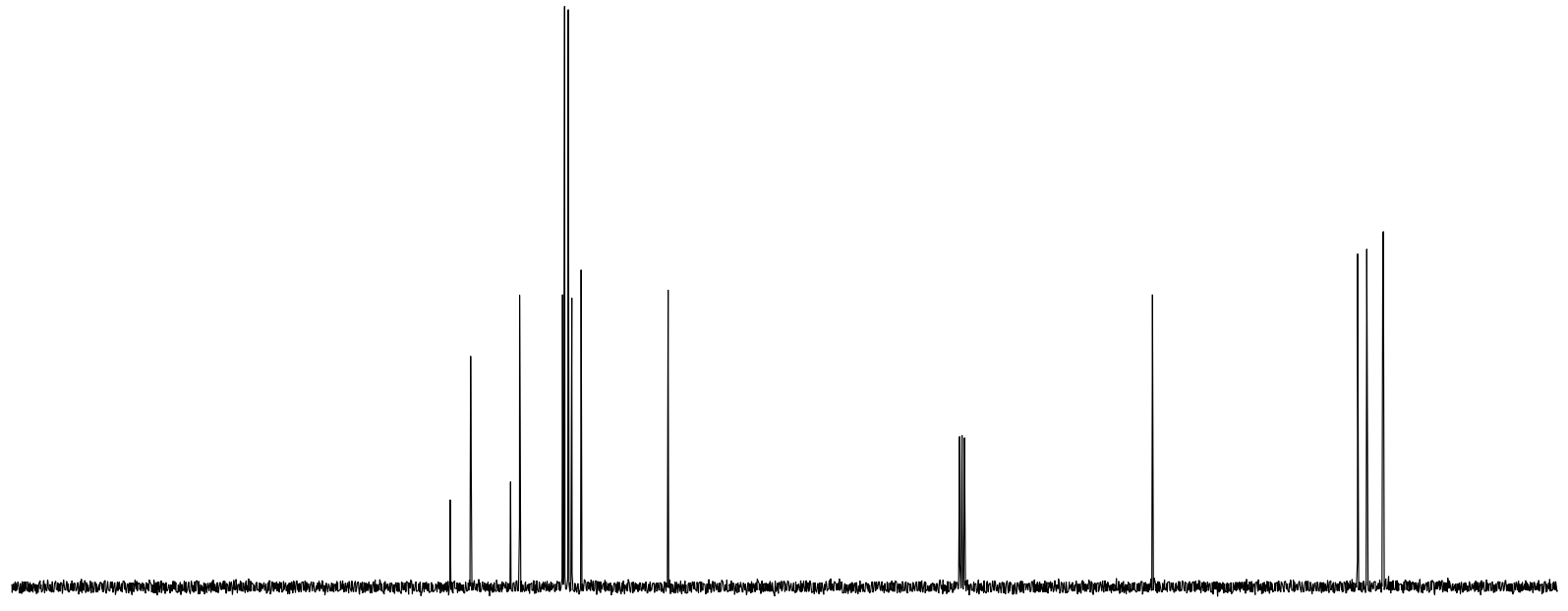

00

180

150

130

120

100
$\mathrm{f} 1(\mathrm{ppm})$

80

$70 \quad 60$

50

40

30

101 
S63

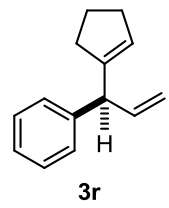

${ }^{1} \mathrm{H}-\mathrm{NMR}: 400 \mathrm{MHz}, \mathrm{CDCl}_{3}$
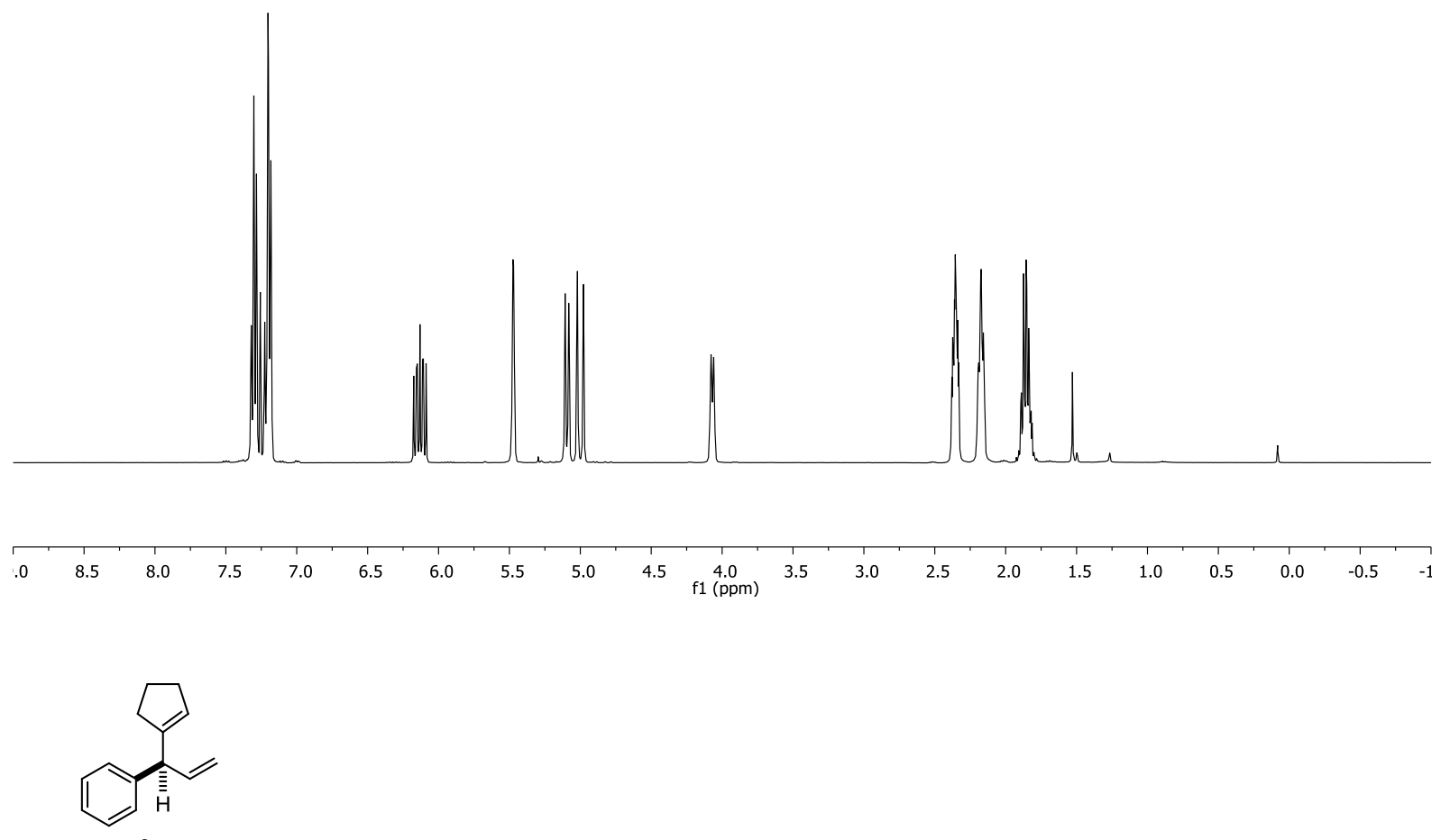

$3 r$

${ }^{13} \mathrm{C}-\mathrm{NMR}: 100 \mathrm{MHz}, \mathrm{CDCl}_{3}$
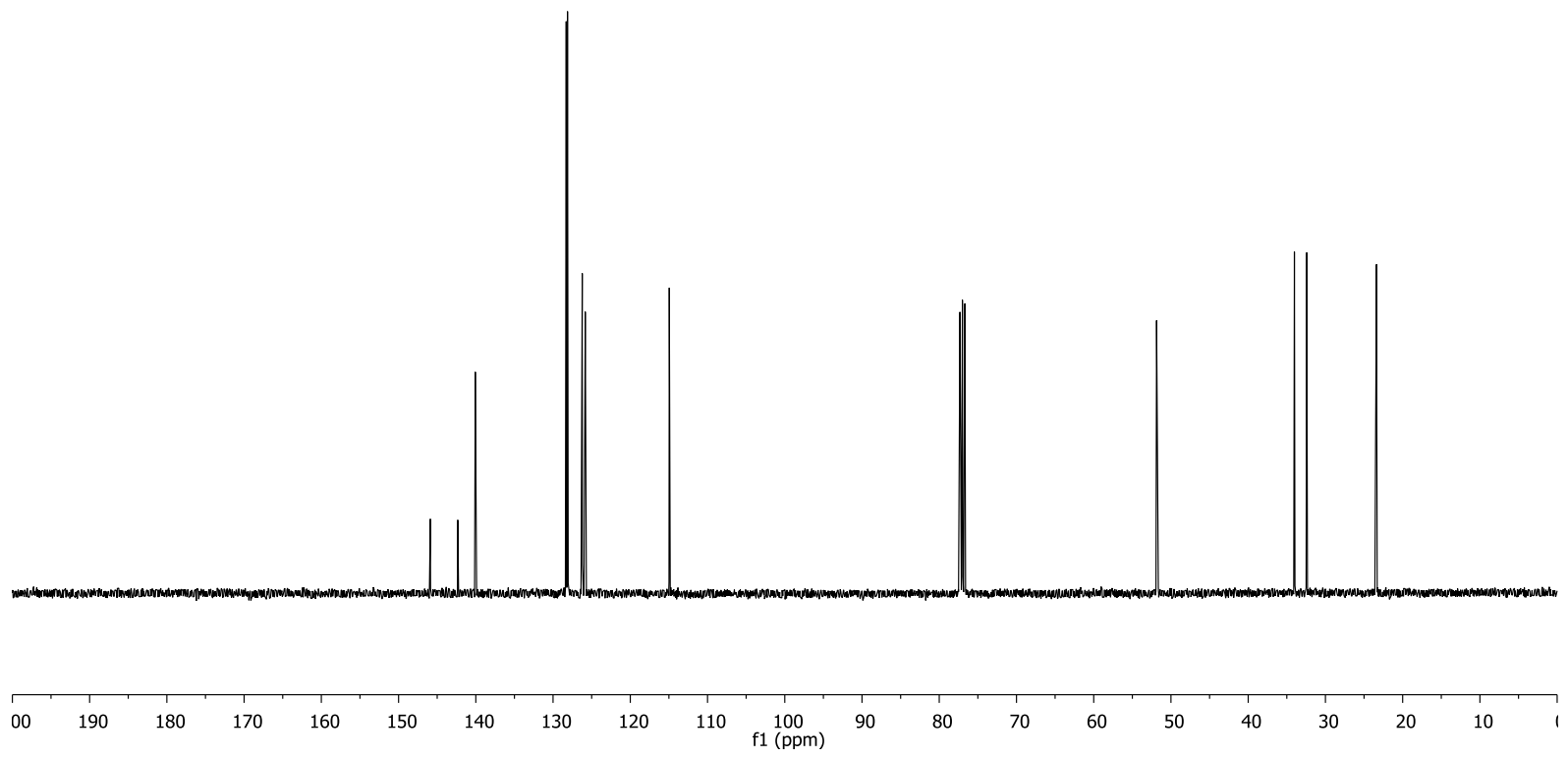
S64

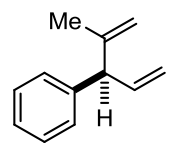

$3 s$

${ }^{1} \mathrm{H}-\mathrm{NMR}: 400 \mathrm{MHz}, \mathrm{CDCl}_{3}$
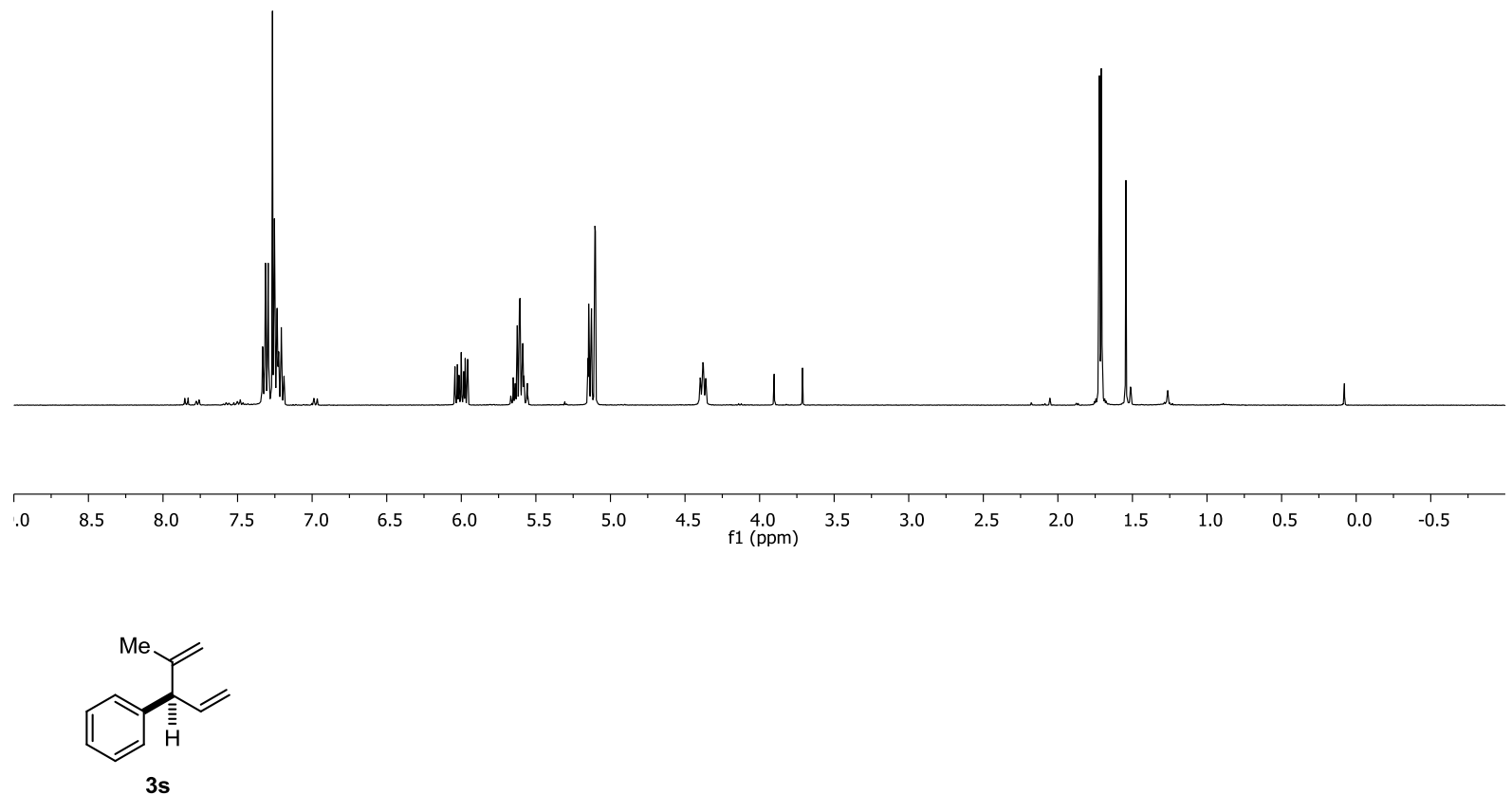

${ }^{13} \mathrm{C}-\mathrm{NMR}: 100 \mathrm{MHz}, \mathrm{CDCl}_{3}$
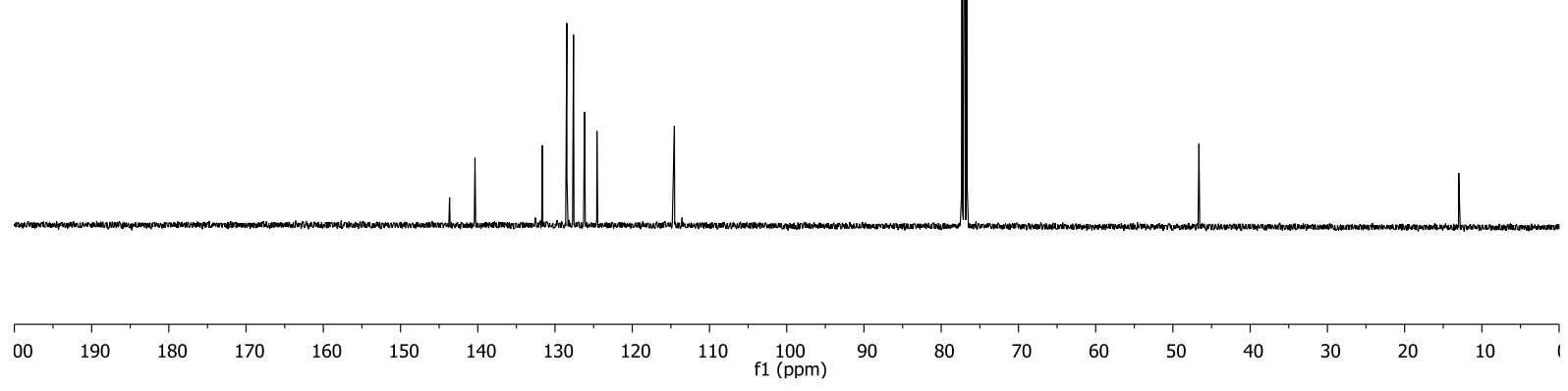


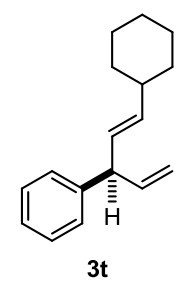

${ }^{1} \mathrm{H}-\mathrm{NMR}: 400 \mathrm{MHz}, \mathrm{CDCl}_{3}$
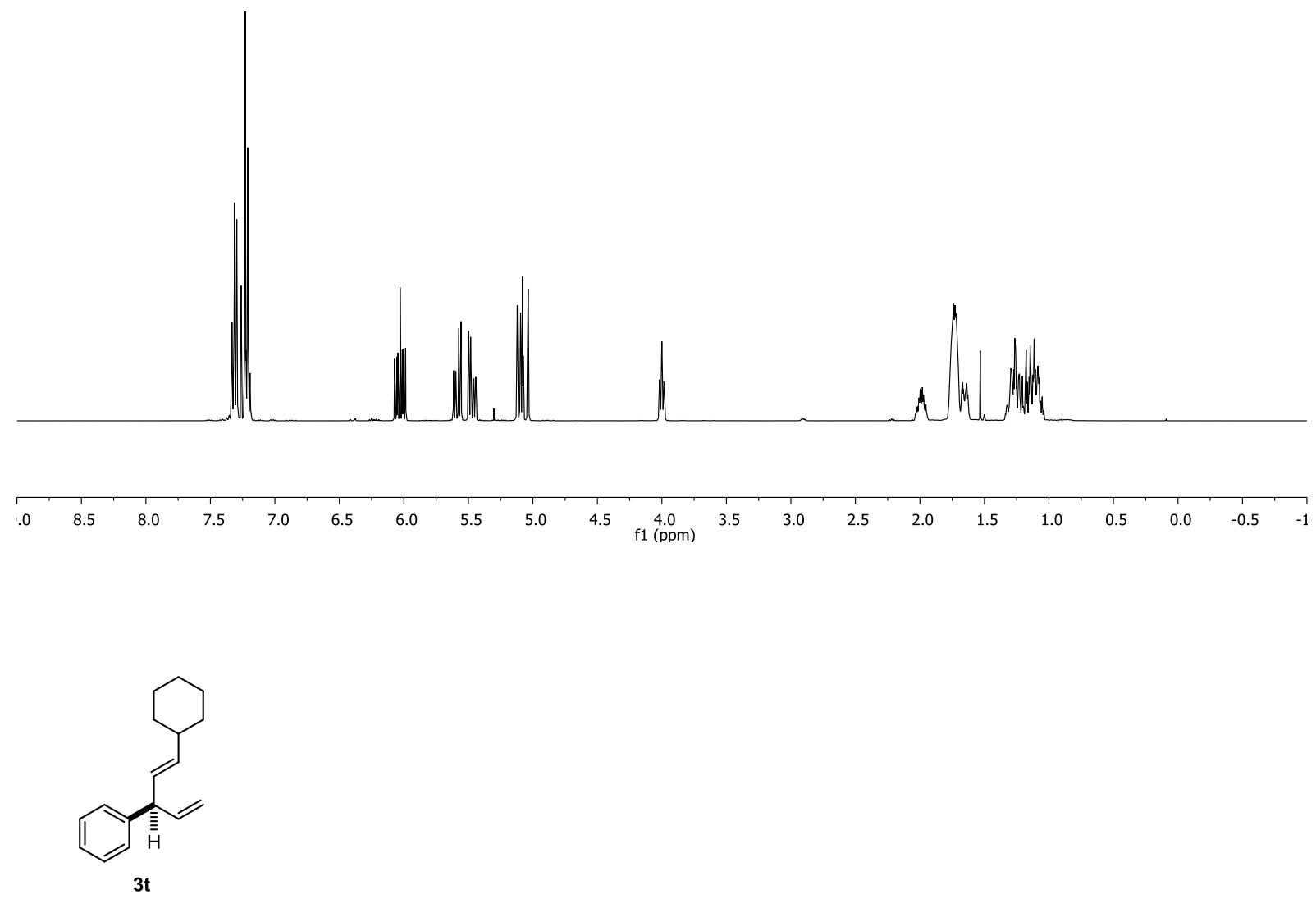

${ }^{13} \mathrm{C}-\mathrm{NMR}: 100 \mathrm{MHz}, \mathrm{CDCl}_{3}$

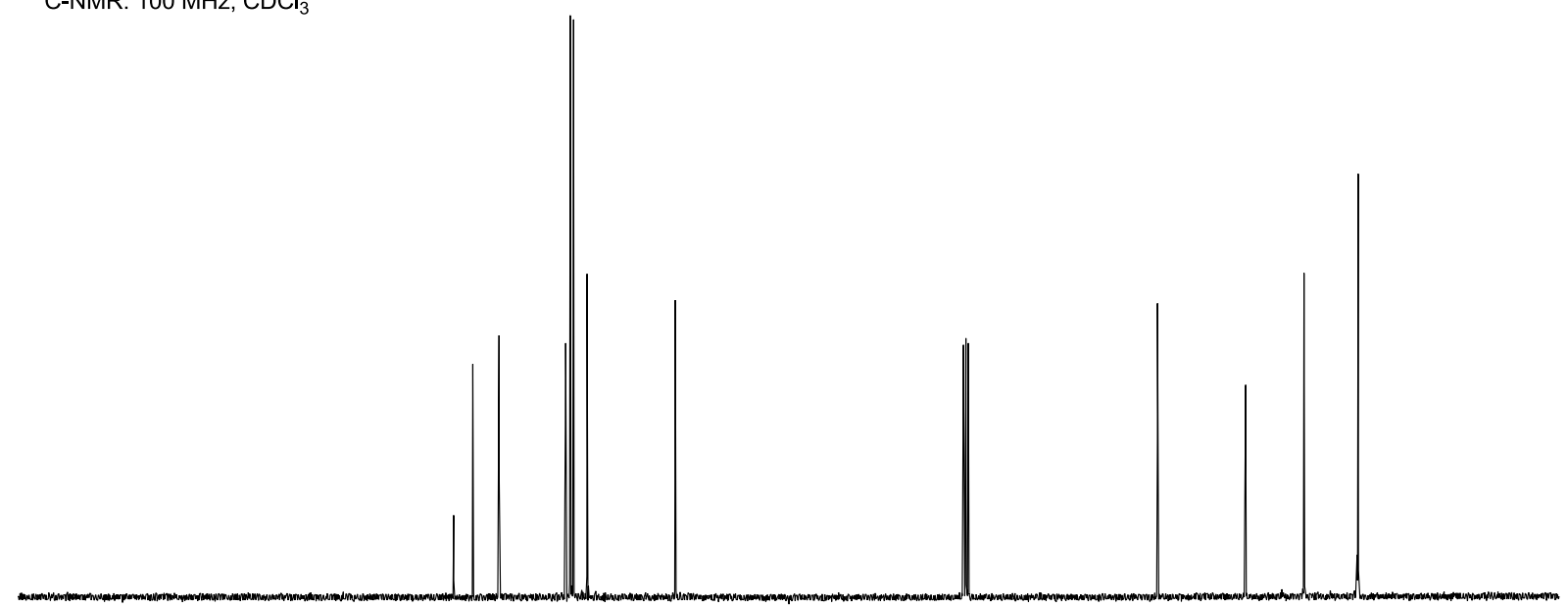


S66

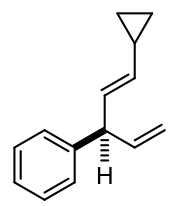

$3 \mathbf{u}$

${ }^{1} \mathrm{H}-\mathrm{NMR}: 400 \mathrm{MHz}, \mathrm{CDCl}_{3}$
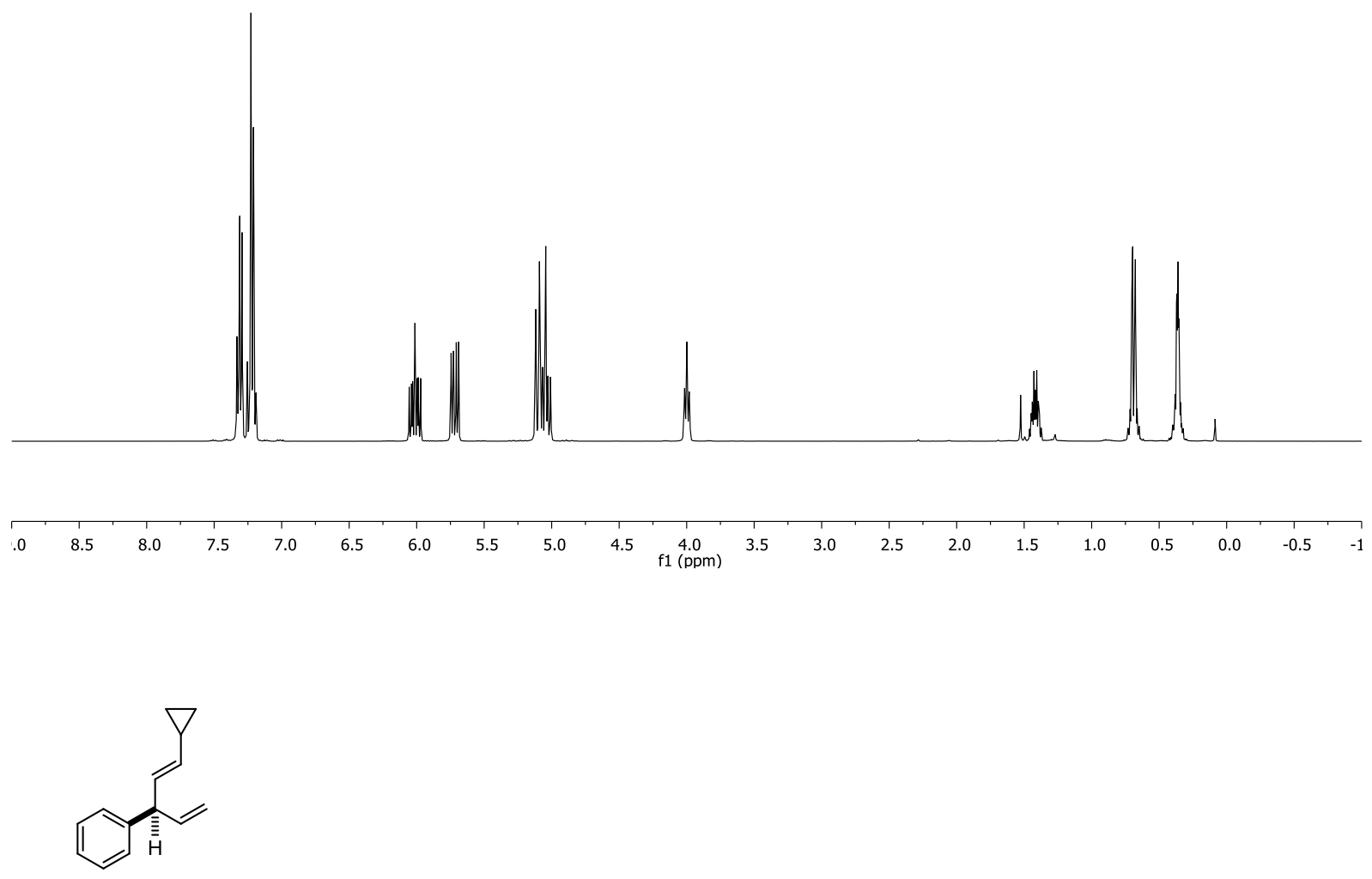

3u

${ }^{13} \mathrm{C}-\mathrm{NMR}: 100 \mathrm{MHz}, \mathrm{CDCl}_{3}$
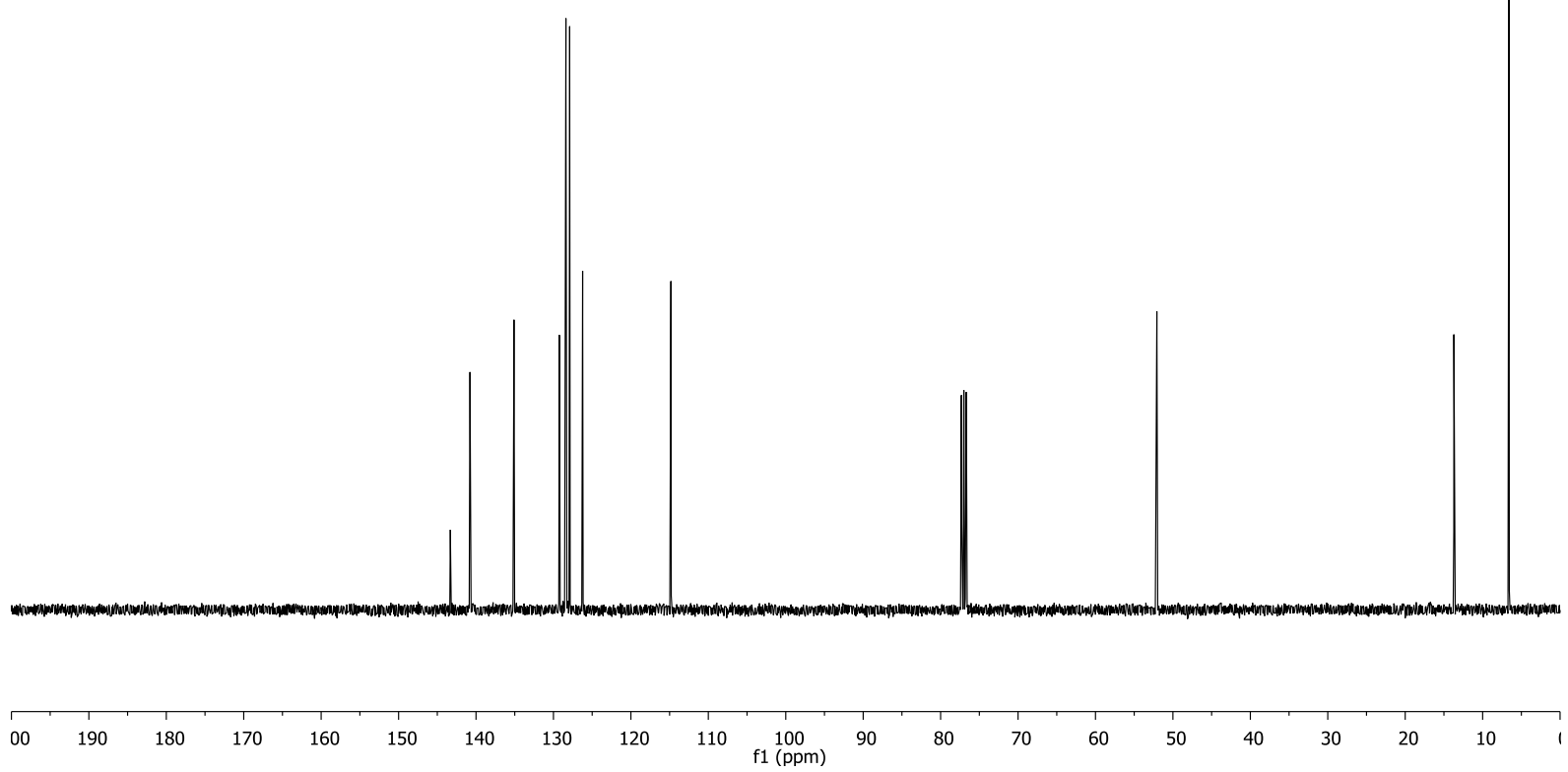


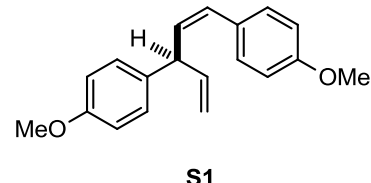

${ }^{1} \mathrm{H}-\mathrm{NMR}: 400 \mathrm{MHz}, \mathrm{CDCl}_{3}$
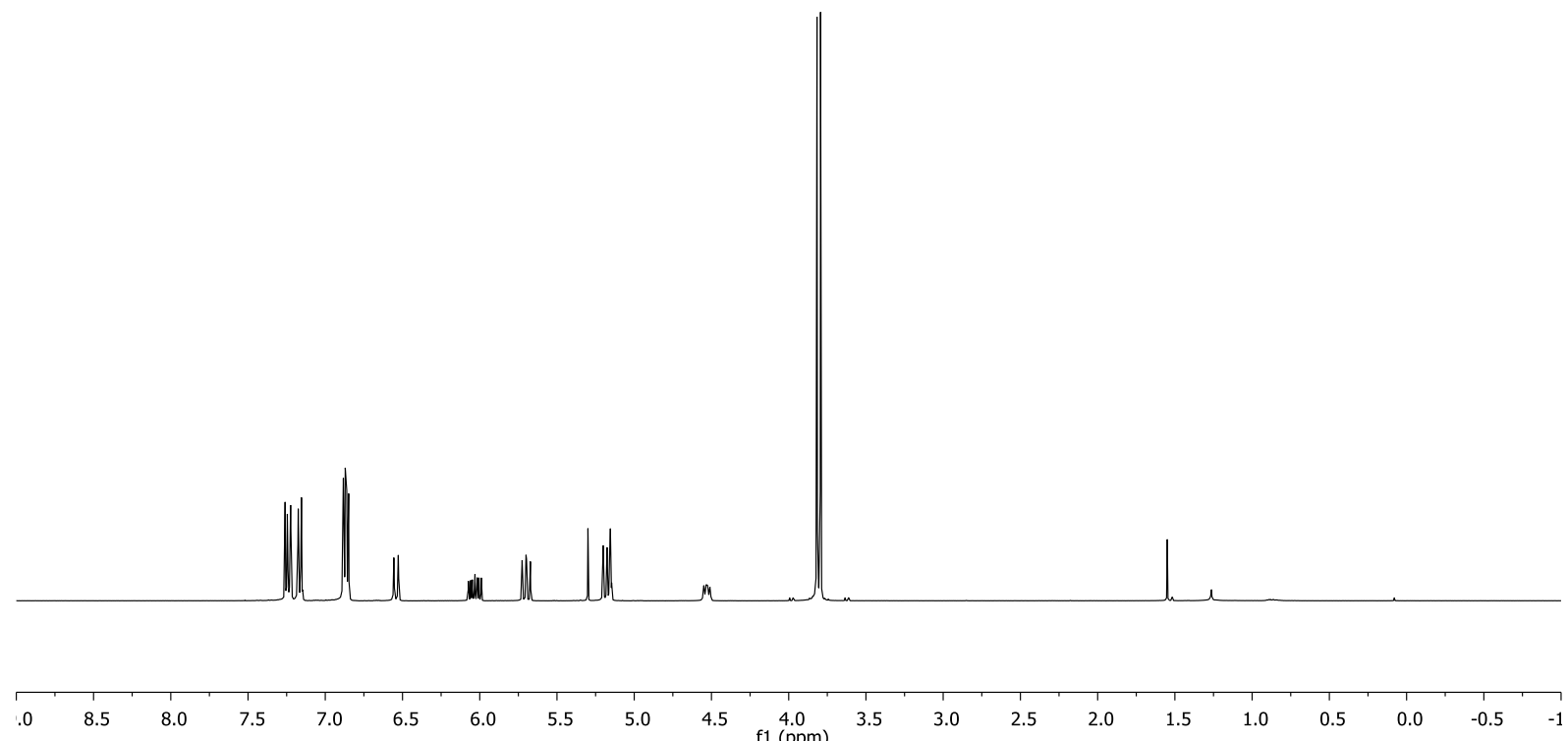<smiles>C=CC(/C=C\c1ccc(OC)cc1)(c1ccc(OC)cc1)c1ccc(OC)cc1</smiles>

s1

${ }^{13} \mathrm{C}-\mathrm{NMR}: 100 \mathrm{MHz}, \mathrm{CDCl}_{3}$

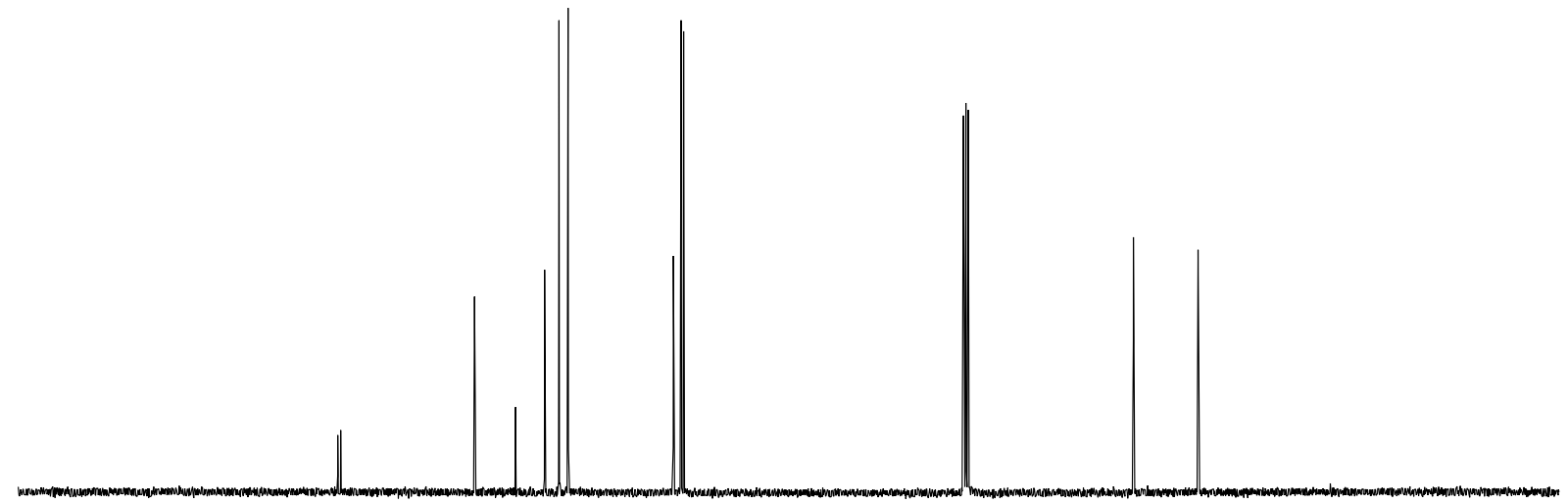$$
00
$$ 
S68

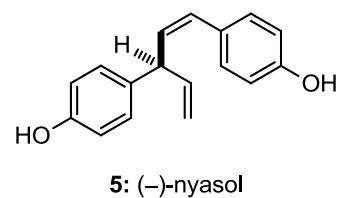

${ }^{1} \mathrm{H}-\mathrm{NMR}: 400 \mathrm{MHz}, \mathrm{CDCl}_{3}$
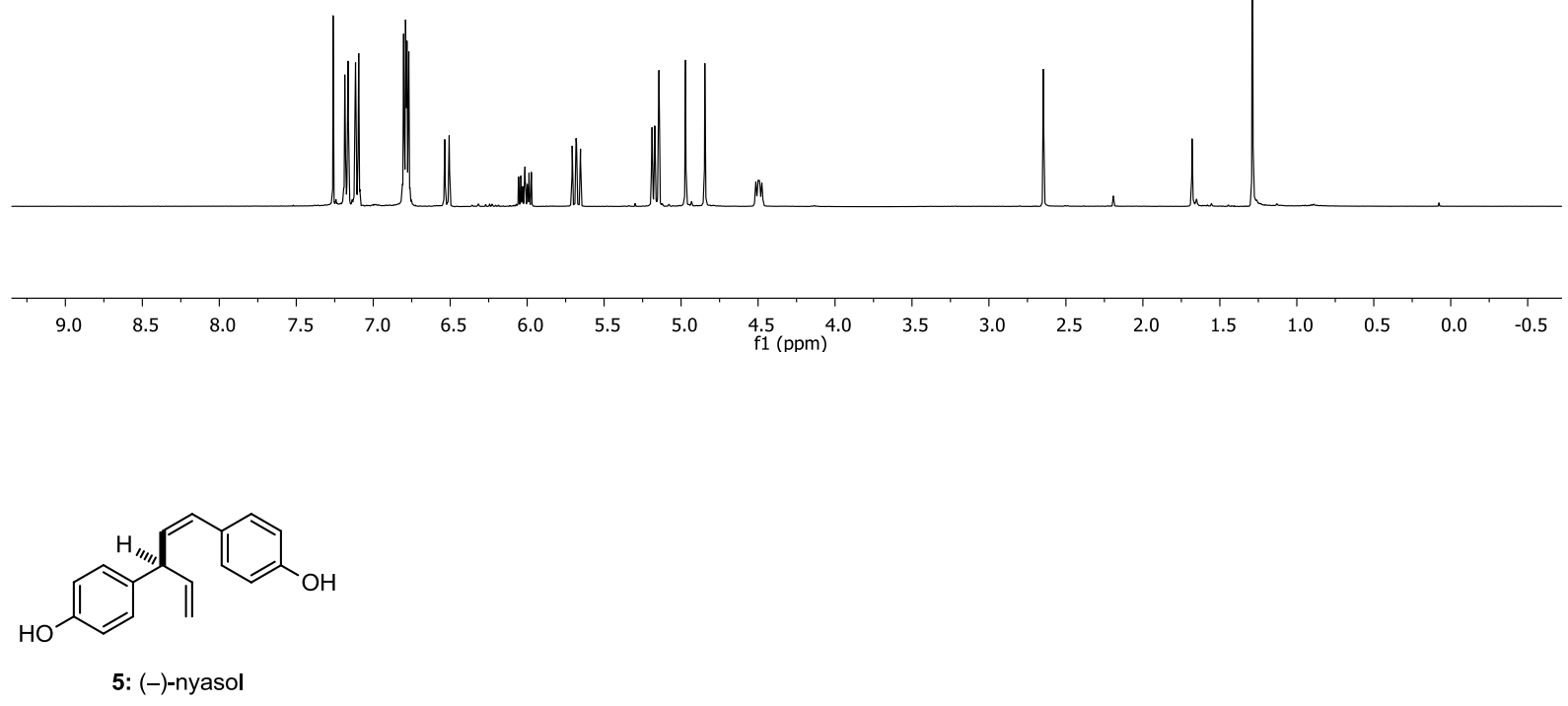

${ }^{13} \mathrm{C}-\mathrm{NMR}: 100 \mathrm{MHz}, \mathrm{CDCl}_{3}$

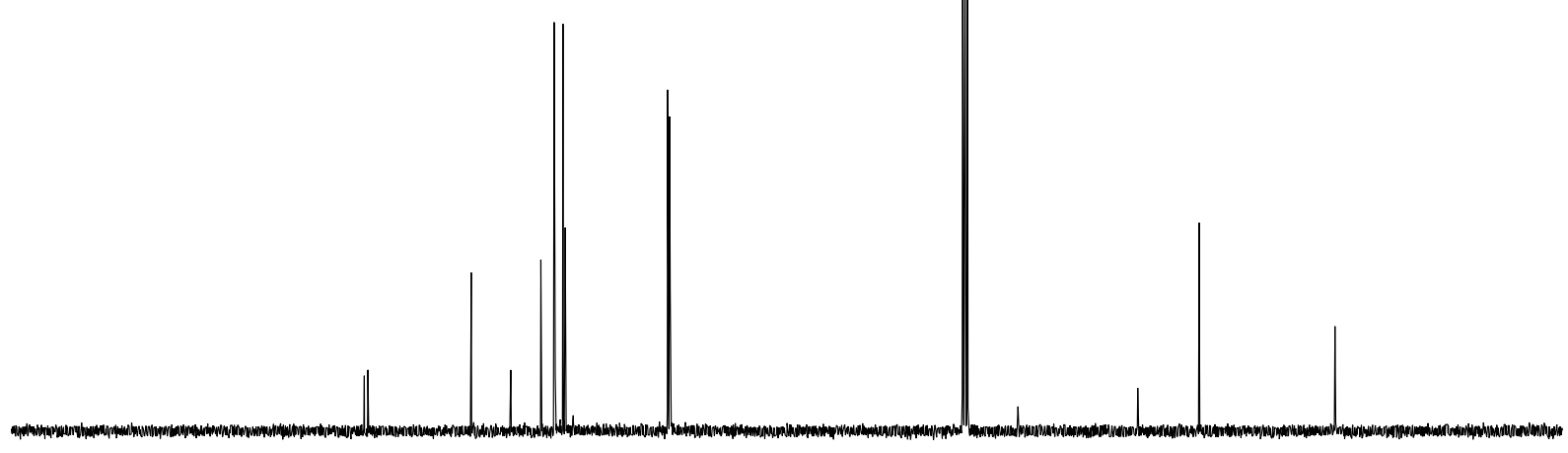

00

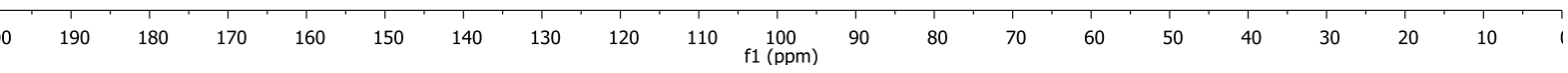




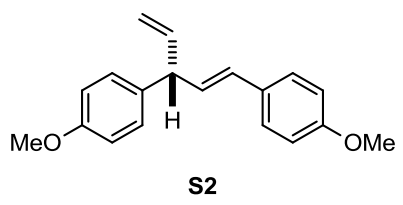

${ }^{1} \mathrm{H}-\mathrm{NMR}: 400 \mathrm{MHz}, \mathrm{CDCl}_{3}$
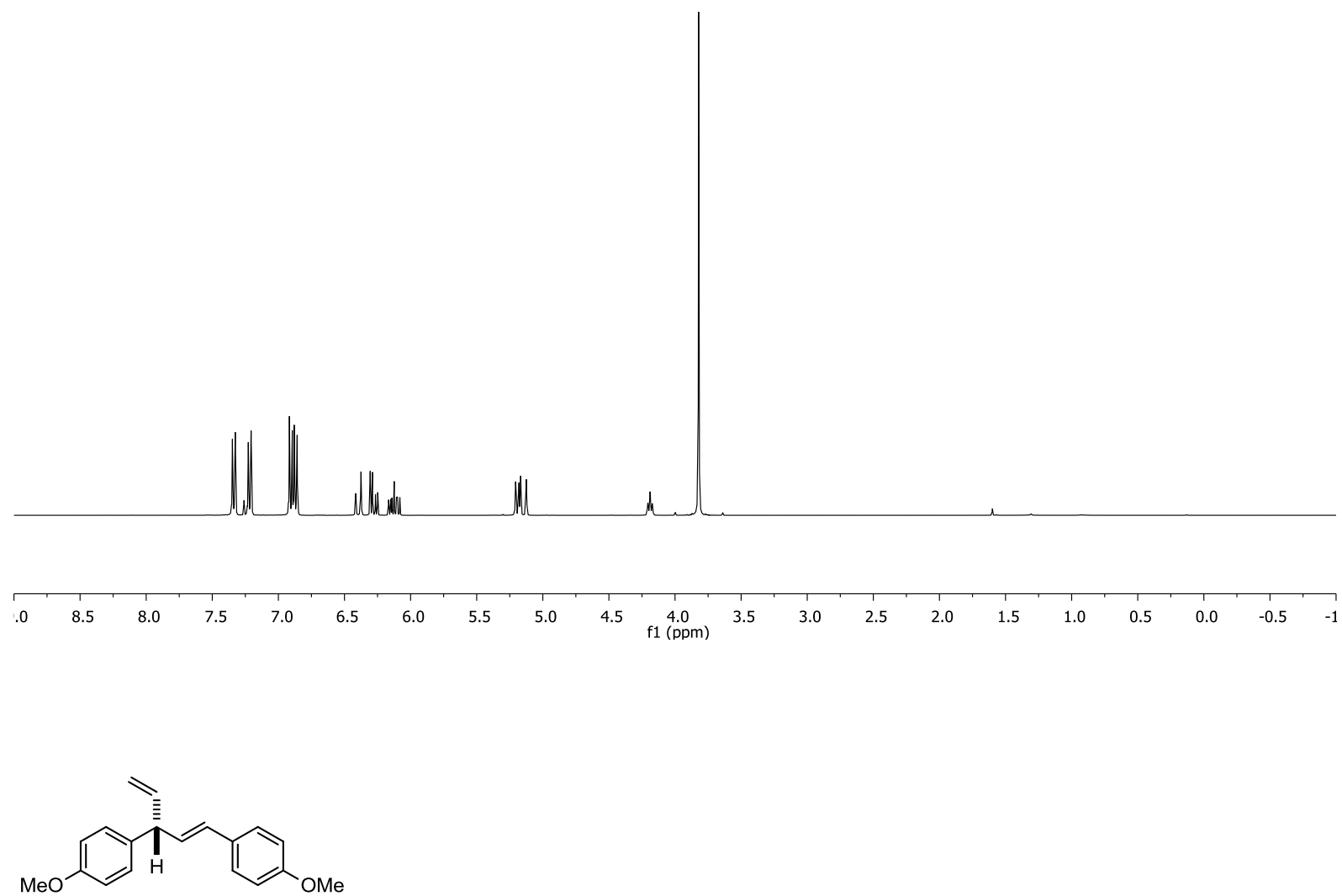

S2

${ }^{13} \mathrm{C}-\mathrm{NMR}: 100 \mathrm{MHz}, \mathrm{CDCl}_{3}$

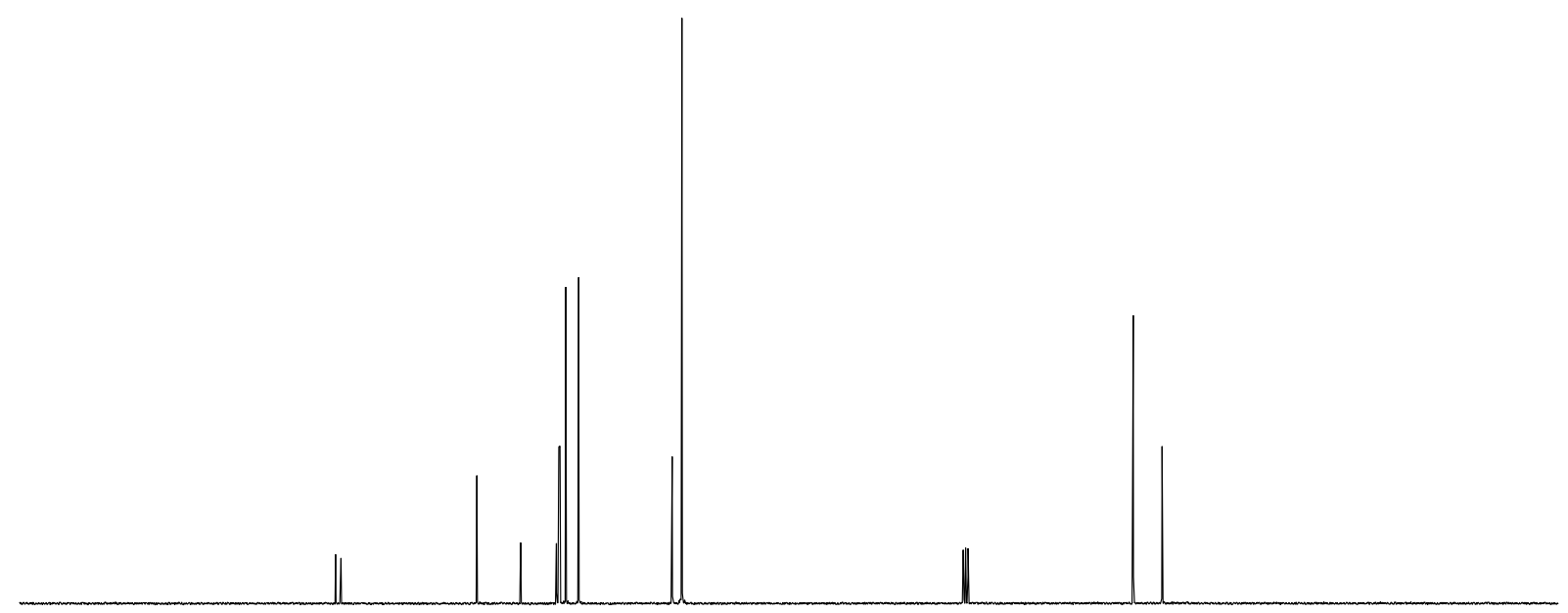

00

$180 \quad 170$

$160 \quad 150$

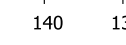

$130 \quad 120 \quad 110 \quad \begin{gathered}100 \\ \mathrm{f} 1(\mathrm{ppm})\end{gathered}$

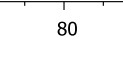

60

$\begin{array}{llllll}50 & 40 & 30 & 20 & 10\end{array}$ 
S70

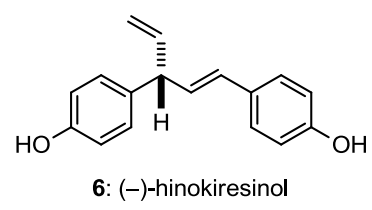

${ }^{1} \mathrm{H}$-NMR: $400 \mathrm{MHz}, \mathrm{CDCl}_{3}$
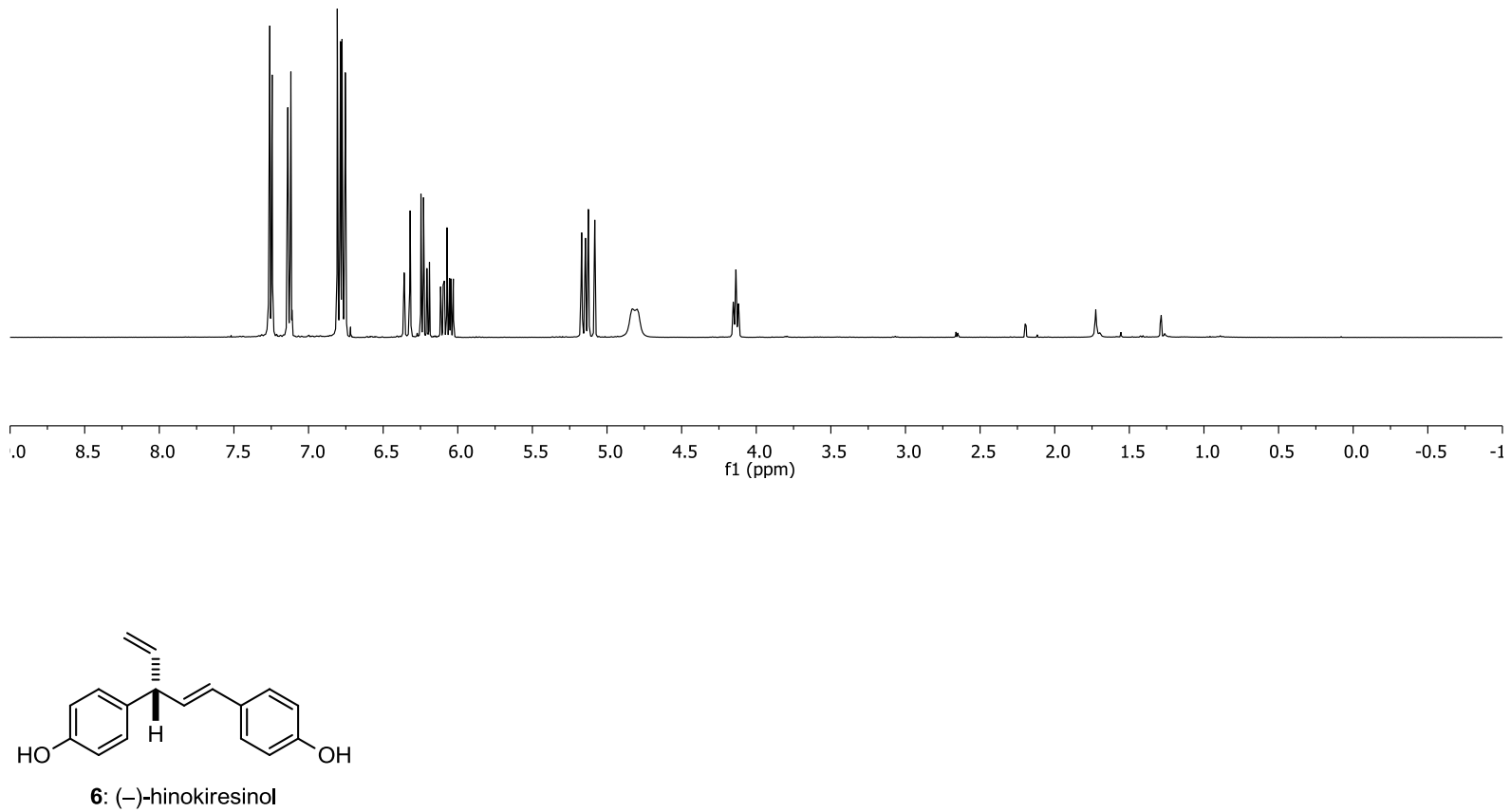

${ }^{13} \mathrm{C}-\mathrm{NMR}: 100 \mathrm{MHz}, \mathrm{CDCl}_{3}$
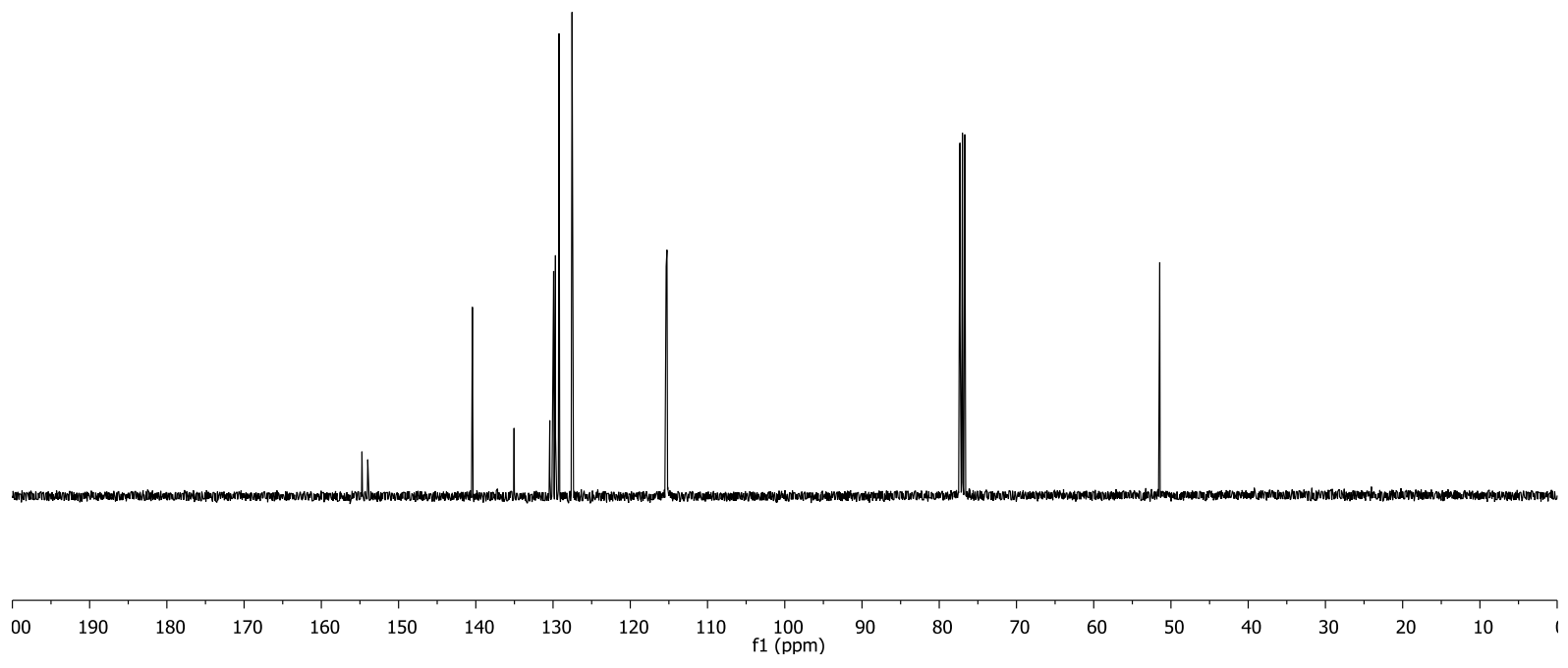